\author{
Monograph \\ urn:1sid:zoobank.org:pub:1F74D019-D13C-426F-835A-24A9A1126C55
}

\title{
Family-group names of fossil fishes
}

\author{
Richard VAN DER LAAN \\ Grasmeent 80, 1357JJ Almere, The Netherlands. \\ Email: richard.vanderlaan80@gmail.com \\ urn:lsid:zoobank.org:author:55EA63EE-63FD-49E6-A216-A6D2BEB91B82
}

\begin{abstract}
The family-group names of animals (superfamily, family, subfamily, supertribe, tribe and subtribe) are regulated by the International Code of Zoological Nomenclature. Particularly, the family names are very important, because they are among the most widely used of all technical animal names. A uniform name and spelling are essential for the location of information. To facilitate this, a list of familygroup names for fossil fishes has been compiled. I use the concept 'Fishes' in the usual sense, i.e., starting with the Agnatha up to the $†$ Osteolepidiformes. All the family-group names proposed for fossil fishes found to date are listed, together with their author(s) and year of publication. The main goal of the list is to contribute to the usage of the correct family-group names for fossil fishes with a uniform spelling and to list the author(s) and date of those names. No valid family-group name description could be located for the following family-group names currently in usage: $\uparrow$ Brindabellaspidae, $\uparrow$ Diabolepididae, $\dagger$ Dorsetichthyidae, $†$ Erichalcidae, $†$ Holodipteridae, $†$ Kentuckiidae, $†$ Lepidaspididae, $†$ Loganelliidae and $\uparrow$ Pituriaspididae.
\end{abstract}

Keywords. Nomenclature, ICZN, Vertebrata, Agnatha, Gnathostomata.

Van der Laan R. 2018. Family-group names of fossil fishes. European Journal of Taxonomy 466: 1-167. https://doi.org/10.5852/ejt.2018.466

\section{Contents}

Introduction

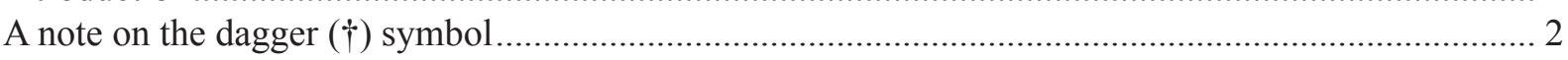

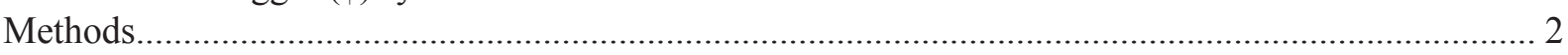

Rules for the family-group names and how the comments are reported in the list ............................... 4

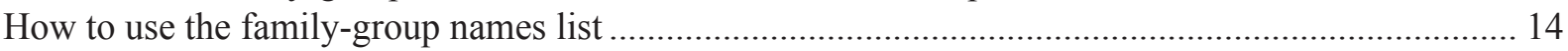

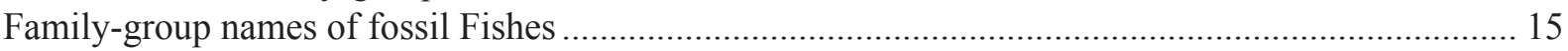

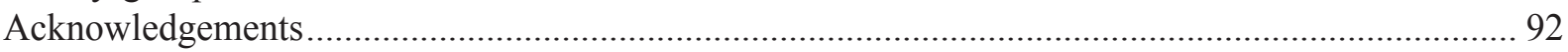

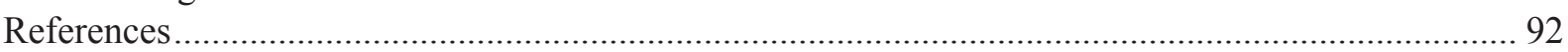

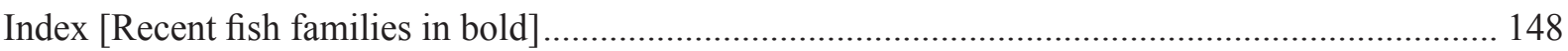

\section{Introduction}

Family names in zoology are useful tools for the storage and retrieval of biological and non-biological information attached to a group of related species and are among the most widely used of all technical names for fossil fishes. 
These zoological family-group names, the names for the family-series: superfamily (suffix -oidea), family (suffix -idae), subfamily (suffix -inae), tribe (suffix -ini) and subtribe (suffix -ina), are regulated by the International Code of Zoological Nomenclature (hereafter named the Code) as published by the International Commission on Zoological Nomenclature (ICZN). The fourth edition of the Code (in effect from 1 January 2000) provides a set of rules for the naming of animals and the resolution of nomenclatural problems. Articles within the Code are called out in the text of this article using the abbreviations Art. or Arts.

The principle of priority also applies to family-group names, with some restrictions (see Van der Laan et al. 2014: 8-9). In order to be available, a family-group name proposed before 1931 must be a scientific name (i.e., in Latinized form) in the nominative plural based on the stem of an available genus name then used as valid in the new suprageneric taxon (Art. 11.7). New family-group names proposed between 1931 and 1999 had to be described in words or associated with a bibliographic reference to such a description in order to be considered available (Arts 13, 15). A family-group name proposed in that time period without a description is available from its original publication (Art. 13.1.3), only if it was used as valid before 2000 and not rejected by an author who, after 1960 and before 2000, expressly applied Art. 13 of the then current editions of the Code (Art. 13.2.1). Family-group names proposed since 2000 have to be explicitly indicated as new and the name of the type genus has to be clearly cited in order to be available (Art. 16).

Given the importance of family names in the scientific literature, it is very important to use uniform family-group names (Van der Laan et al. 2014: 3). A uniform name and spelling diminishes the trouble of finding the information connected with the family name. A single spelling of the stem is also important for having uniform names in the order series. For example, it is somewhat confusing to state the family $\dagger$ Astraspidae (with stem Astrasp-) as belonging to the order $\uparrow$ Astraspidiformes (with stem Astraspid-).

Locating the widely scattered family-group names in the literature is not easy and workers have often ignored priority of these names. A lot of works do not have accurate authors and dates for families and subfamilies. For easy access it is useful to have accurate family-group name lists (Van der Laan et al. 2014; see for more examples Bouchet \& Rocroi 2005 or Bouchard et al. 2011).

Family-group names of Recent fishes were published by Van der Laan et al. (2014) and are regularly updated (e.g. Van der Laan 2018).

\section{A note on the dagger ( + symbol}

Fossils can sample lineages close in time to relatively deep splitting events. They may bridge problematic morphological gaps and display combinations of character states that are not found in the extant biota, thereby subdividing long branches and improving phylogenetic accuracy (Cobbett et al. 2007; Edgecombe 2010). To make use of this advantage, many studies use fossil taxa in combination with Recent taxa in phylogenetic analyses (e.g., Grande \& Bemis 1991, 1998; Santini \& Tyler 2003, 2004; Grande \& Hilton 2006; Davesne et al. 2014; Bemis et al. 2018). When fossil fishes appear alongside living fishes in the same classification scheme, it is important to distinguish if a certain taxon contains fossil or Recent fishes, or both. This distinction conveys the meaning that the information about the fossil taxon is necessarily less complete than it would be for an extant taxon (Bemis 2016: 321). Thus, in this work, I use the dagger symbol " $\uparrow$ " preceding the scientific name, to denote all taxa containing exclusively fossil fishes.

\section{Methods}

The concept 'Fishes' is used in the usual, non-monophyletic sense (Bemis 2016: 312), i.e., starting with Agnatha and including all non-tetrapods. Although Fowler (1964: 20) included the lancelets in 
his famous A Catalog of World Fishes, these fish-like marine chordates are nowadays classified in the subphylum Cephalochordata and no longer included in 'Fishes'. The debate over conodont relationships still remains a somewhat open question and I have not included the conodont family names in this list of 'Fishes'.

As with the family-group names for Recent fishes (Van der Laan et al. 2014), problems were encountered with the priority of some names for fossil fishes and with the spelling of certain family names. Some seniority problems can be easily solved by applying the Code, but there are many senior 'overlooked / forgotten' names that are not in use. Changing a well-established family name to a little-known older name (although in line with the present Code), will seriously upset the classification and cause unneeded confusion. In this list, the practical concept of prevailing recent practice is followed (Van der Laan et al. 2014).

For example, the family-group name $\dagger$ Crossognathidae Woodward, 1901 is a well-known family of fossil fishes (hundreds of citations). However, earlier in 1901, Cragin (1901) proposed two familygroup names, $\uparrow$ Syllaemidae and $\uparrow$ Pelecorapidae. As long as the type genera are thought to belong to the same family, one of the names of Cragin has priority ( $†$ Pelecorapidae was selected by the first revisor). Art. 23.9 Reversal of Priority cannot be evoked, because both senior names are used after 1899 (Art. 23.9.1.1). Changing the familiar family name $\uparrow$ Crossognathidae (also echoed in the name of the order $\uparrow$ Crossognathiformes) is clearly undesirable. The formal solution is to appeal to the Commision (Art. 23.9.3). With the practical concept of prevailing recent practice, the time-consuming appeal process can be avoided. In contrast to the names of species, where 'stability is ignorance' (Raposo et al. 2017), the names and spelling of families can easily be stabilized.

The spelling of family-group names ending in -aspidae or -aspididae (genus name ending in -aspis), -lepidae or -lepididae (genus name ending in -lepis) and -odidae or -odontidae (genus name ending in -odus) is a source of confusion. Besides the arguments expressed in Van der Laan et al. (2014), I think it is probably best to follow the advice of Woodward (1891a: xxii), Gill (1893: 129) and Jarvik (1985: 10) to use the shorter version for clarity and simplicity, unless the longer version is in prevailing recent practice (see Van der Laan et al. 2014: 4). However, there is a definite trend in the literature to use the longer family names. When in doubt which of the spellings is used more often, it is probably best to retain the original spelling.

Another problem is the lack of a database for the species of fossil fishes, equivalent to the Catalog of Fishes for Recent fishes (Eschmeyer et al. 2018).

Searching for the correct author / date combination of a fossil fish genus, its validity, and its current systematic placement required a great deal of time and in some cases proved insufficient. I checked all the type genera in Neave's Nomenclator Zoologicus with the help of Internet and generally follow the given describer(s) and date. As already noted by Bemis (2016: 323), a dedicated, comprehensive and scholarly Catalog of Fossil Fishes, that has been carefully interpreted using the Code, is sorely missed.

Internet was searched with the help of:

- http://ubio.org/NomenclatorZoologicus/ [Neave S.A. Nomenclator Zoologicus, 10 Volumes, names published from 1758 to 2004];

- http://www.itis.gov/ [Integrated Taxonomic Information System];

- http://fossilworks.org/ [Gateway to the Paleobiology Database];

- http://www.organismnames.com/query.htm [Index to Organism Names (ION)];

- http://www.gbif.org/ [Global Biodiversity Information Facility, Vanhoorne 2017];

- http://taxonomicon.taxonomy.nl/Default.aspx [the Taxonomicon \& Systema Naturae 2000]; 
- http://palaeos.com/vertebrates/dendrograms/chordata.html [Palaeos, a multi-authored encyclopedia on the history of life on Earth];

- https://shark-references.com/ [Pollerspöck J. \& Straube N. 2015, Bibliography database of living/ fossil sharks, rays and chimaeras (Chondrichtyes: Elasmobranchii, Holocephali)].

The family-group names of fishes are presented in several modern classifications of the 'Fishes' (Wiley \& Johnson 2010; Long 2011; Betancur-R. et al. 2013; Westheide \& Rieger 2015; Nelson et al. 2016; Betancur-R. et al. 2016, 2017; Cavin 2017; Hughes et al. 2018). Phylogenetic relationships of basal vertebrates have not yet reached a stable consensus. For the higher classification to be somewhat comparable, I also consulted the classification used in the series Handbook of Paleoichthyology (Denison 1978, 1979; Zangerl 1981; Cappetta 1987, 2012; Stahl 1999; Märss et al. 2007; Ginter et al. 2010), somewhat modified based on the classification put forward by Nelson et al. (2016). They present higher ranks at a somewhat lower rank than does the Catalogue of Life initiative (Ruggiero et al. 2015).

Because there are few comprehensive genus-based phylogenies of fossil fishes, the phylogenetic framework used here is a compilation of phylogenies found in the literature for each order, family and genus. The family-group names are indexed, so searching for a possible family-group name based on a genus (but placed in a different family in this work), should be straightforward.

If the systematic placement is in doubt (taxonomic groups that are difficult to place with any degree of certainty), then I have listed the family-group names alphabetically under a higher rank with the remark incertae sedis.

If the name is dubious and has not recently been used as a separate family (or nowadays not used at all), I simply state the name in one line. If the name is dubious, but has recently been used as a separate family, then the name is stated as a separate family.

As the rules of the ICZN do not require the use of commas in the authorship, they were left out in this paper for the family-group names and the type genera. All authorships of family-group names have been included in the bibliography.

The more than eleven-hundred family-group names in the list below are presented in the following way:

Original name and spelling [correction of obvious type-setting error] author year: page number (rank) type Genus author year [comments on spelling / correction of the stem / availability / validity].

Synonymous family-group names are listed according to decreasing priority under the valid family name. I do not list all the invalid vernacular or non-typical names used for fossil fishes. Moreover, some very old family names that are nowadays used exclusively in the ordinal series are not listed.

Every stem of the family name found in the literature has been mentioned, so electronically searching with the stem should find a proposed family-group name.

\section{Rules for the family-group names and how the comments are reported in the list}

The important articles of the Code for the family-group names as applied herein are listed below. I came across so many errors in the family-group names that I found it useful to quote most articles in full (sometimes with comments inserted in brackets). I have deleted a few instances where the sections of the code were repeated elsewhere or were unnecessary (see The Code Online, http://iczn.org/iczn/index.jsp, for the entire document). In between the articles (between $<<>>$ ) are explanations of how I report my findings in the family-group names list. 
The International Commission on Zoological Nomenclature has recently expanded and refined the methods of publication allowed by the Code, particularly in relation to electronic publication. The amendment allows electronic publication after 2011 under certain conditions and disallows publication on optical discs after 2012. The requirements for electronic publications are that the work be registered in ZooBank before it is published, that the work itself state the date of publication and contain evidence that registration has occurred, and that the ZooBank registration state both the name of an electronic archive intended to preserve the work and the ISSN or ISBN associated with the work.

Article 11.6. Publication as a synonym. A name which when first published in an available work was treated as a junior synonym of a name then used as valid is not thereby made available.

11.6.1. However, if such a name published as a junior synonym had been treated before 1961 as an available name and either adopted as the name of a taxon or treated as a senior homonym, it is made available thereby but dates from its first publication as a synonym.

11.6.3. A name first published after 1960 and treated as a junior synonym on that occasion cannot be made available from that act under Art. 11.6.

$<<$ If a family-group name was published as a synonym and is not available, I add the remark: [name in synonymy; treated as available before 1961?; not available, Art. 11.6.1]. Or I add: [published after 1960 as a junior synonym; not available, Art. 11.6.3].>>

Article 11.7.1. A family-group name when first published must meet all the following criteria. It must:

11.7.1.1. be a noun in the nominative plural formed from the stem of an available generic name [Art. 29, this generic name is called the type genus Art. 63] (indicated either by express reference to the generic name or by inference from its stem, but for family-group names proposed after 1999 see Art. 16.2); the generic name must be a name then used as valid in the new family-group taxon [Arts 63-64] (use of the stem alone in forming the name is accepted as evidence that the author used the generic name as valid in the new family-group taxon unless there is evidence to the contrary).

$<<$ If the family-group name is not formed from the stem of an available generic name I add the remark: [no stem of the type genus, not available, Art. 11.7.1.1]. I only list some old atypical names.

If the family-group name is formed from the stem of a, not used as a valid, available generic name I add: [no valid type genus, not available, Art. 11.7.1.1]. This usage is sometimes difficult to find (and easily missed!) as the synonymy of the type genus is sometimes in a different part of the publication.

If the type genus is not mentioned, but the stem of an available generic name has been used in forming the name and there is no evidence of invalidity of the type genus, I add: [type genus inferred from the stem, Art. 11.7.1.1], and I treat the name as available.

If an author published a few papers mentioning the invalidity of the potential type genus before and after the publication with the new family-group name, then I determined there was evidence to the contrary (Art. 11.7.1.1), and I add: [no valid type genus, not available, Art. 11.7.1.1]. If it is evident from the publication that an author listed all of the valid genera and the type genus is not among them, then I also determined there was evidence to the contrary (Art. 11.7.1.1), and I add: [no valid type genus, not available, Art. 11.7.1.1].

If the type genus is not mentioned and the inference is questionable, I add a question mark before the family-group name (and sometimes add: [family uncertain] if the systematic position of the familygroup name is uncertain), and I treat the name as not available. $>>$ 
11.7.1.2. be clearly used as a scientific name to denote a suprageneric taxon and not merely as a plural noun or adjective referring to the members of a genus.

$<<$ If an author used the nominative singular to denote a new family-group name (against Art. 11.7.1.1), but it is otherwise very obvious that the author used the name for a suprageneric taxon (for multiple genera), I treat the name as available.

If merely a plural noun I add the remark: (no family-group name). I only list those plural nouns that were referred to by some author as a potential family-group name.>>

11.7.1.3. end with a family-group name suffix except as provided in Art. 11.7.2; a family-group name of which the family-group name suffix [Art. 29.2] is incorrect is available with its original authorship and date, but with a corrected suffix [Arts 29, 32.5.3];

11.7.1.4. not be based on certain names applied only to fossils and ending in the suffix -ites, -ytes or -ithes [Art. 20];

11.7.1.5. not be based on a genus-group name that has been suppressed by the Commission [Art. 78].

11.7.2. If a family-group name was published before 1900, in accordance with the above provisions of this article but not in latinized form, it is available with its original author and date only if it has been latinized by later authors and has been generally accepted as valid by authors interested in the group concerned and as dating from that first publication in vernacular form.

$<<$ If the family-group name is not proposed in a latinized form and has been latinized by later authors I add the remark: [latinized to \{family-group name\} by \{author year: page number\}]. If the familygroup name has been generally accepted as valid by authors interested in the group concerned and as dating from that first publication in vernacular form, I add: [considered valid with this authorship by \{author year: page number\} Art. 11.7.2]. If I state both provisions, then I treated the name as available.

For fossil fishes, it is very difficult to find authors who mention the author(s)/date of family-group names. For fishes, Art. 11.7.2 can be difficult and lead to different opinions. I tried to establish the most accepted author(s)/ date combination.

If not treated as valid and as dating from the first publication in vernacular form, I add: [published not in latinized form before 1900, not available, Art. 11.7.2]. I list only some early vernacular names that possibly could have been latinized by a later author, and I do not present an exhaustive list of all the vernacular family-group names.

If the family-group name is not proposed in a latinized form after 1899, I add: [published not in latinized form after 1899, not available].>>

\section{Article 12. Names published before 1931}

12.1. Requirements. To be available, every new name published before 1931 must satisfy the provisions of Art. 11 and must be accompanied by a description or a definition of the taxon that it denotes, or by an indication.

12.2. Indications. For the purposes of this article the word 'indication' denotes only the following:

12.2.1. A bibliographic reference to a previously published description or definition even if the description or definition is contained in a work published before 1758 , or that is not consistently binominal, or that 
has been suppressed by the Commission (unless the Commission has ruled that the work is to be treated as not having been published [Art. 8.7]);

12.2.2. The inclusion of a name in an index to a work that is not consistently binominal, provided that the provisions of Art. 11.4.3 are satisfied;

12.2.3. The proposal of a new replacement name (nomen novum) for an available name, whether or not required by any provision of the Code;

12.2.4. The formation of a family-group name from an available generic name [Art. 29].

\section{Article 13. Names published after 1930}

13.1. Requirements. To be available, every new name published after 1930 must satisfy the provisions of Art. 11 and must

13.1.1. be accompanied by a description or definition that states in words characters that are purported to differentiate the taxon, or

13.1.2. be accompanied by a bibliographic reference to such a published statement, even if the statement is contained in a work published before 1758, or in one that is not consistently binominal, or in one that has been suppressed by the Commission (unless the Commission has ruled that the work is to be treated as not having been published [Art. 8.7]), or

13.1.3. be proposed expressly as a new replacement name (nomen novum) for an available name, whether required by any provision of the Code or not.

13.2. Family-group names. To be available, every new family-group name published after 1930 must satisfy the provisions of Art. 13.1 and must be formed from an available genus-group name then used as valid by the author in the family-group taxon [Arts 11.7.1.1 and 29].

13.2.1. A family-group name first published after 1930 and before 1961 which does not satisfy the provisions of Art. 13.1 is available from its original publication only if it was used as valid before 2000, and also was not rejected by an author who, after 1960 and before 2000, expressly applied Art. 13 of the then current editions of the Code.

13.5. Combined description of new family-group taxon and new genus. The combined description or definition of a new nominal family-group taxon and a single new nominal genus of which the name provides the basis for the new family-group name [Art. 11.5] is deemed to confer availability on each name under Art. 13.1.1, but for such names published after 1930 availability is not conferred on either name unless a type species is fixed for the new nominal genus [Arts 13.2 and 13.3].

$<<$ If there is no description of the taxon, I add the remark: [name only]. If the family-group name was published before 1961 and if used as valid before 2000, I add: [name only, but used as valid by \{author year\}: page number Art. 13.2.1], and I treat the name as available. If I could not find a validating usage, I add: [name only, used as valid before 2000?; not available].

If the family-group name was published before 1961 and has been rejected with the help of Art. 13 after 1960 and before 2000, I add: [name only, rejected by \{author year\}: page number with the help of Art. 13; not available]. If the family-group name was published after 1960, I add: [name only, published after 1960, not available, Art. 13.1.1].>> 
Article 16. Names published after 1999

16.1. All names: intention of authors to establish new nominal taxa to be explicit. Every new name published after 1999, including new replacement names (nomina nova), must be explicitly indicated as intentionally new.

16.2. Family-group names: type genus to be cited. In addition to satisfying the provisions of Arts 13-15, a new family-group name published after 1999 must be accompanied by citation of the name of the type genus (i.e., the name from which the family-group name is formed).

$<<$ If a family-group name proposed after 1999 does not satisfy the provisions of Arts 13-16, I add the remark: [not published according to the rules, not available]. In most cases the publication lacks an exact description or definition that states in words characters that are purported to differentiate the taxon or the indication 'new' is absent.>>

\section{Article 23. Principle of Priority}

23.1. Statement of the Principle of Priority. The valid name of a taxon is the oldest available name applied to it, unless that name has been invalidated or another name is given precedence by any provision of the Code or by any ruling of the Commission. For this reason priority applies to the validity of synonyms [Art. 23.3], to the relative precedence of homonyms [Arts 53-60], the correctness or otherwise of spellings [Arts 24, 32], and to the validity of nomenclatural acts (such as acts taken under the Principle of the First Reviser [Art. 24.2] and the fixation of name-bearing types [Arts 68-69, 74.1.3, 75.4]).

23.1.1. For exceptions for certain family-group names see Arts 35.5 and 40 .

23.1.2. For the case of disused family-group names which are homonyms see Art. 55.3.1.1.

23.2. Purpose. In accordance with the objects of the Code (see Preamble), the Principle of Priority is to be used to promote stability and it is not intended to be used to upset a long-accepted name in its accustomed meaning by the introduction of a name that is its senior synonym or homonym (for certain such cases see Art. 23.9), or through an action taken following the discovery of a prior and hitherto unrecognized nomenclatural act (such as a prior type fixation; for such cases see Arts 70.2 and 75.6).

23.3.1. Priority of the name of a nominal taxon is not affected by elevation or reduction in rank of the taxon within the family group, genus group or species group [Arts 36, 43, 46], nor by any mandatory change in suffix of a family-group name consequent upon change in rank [Art. 34].

23.9. Reversal of precedence. In accordance with the purpose of the Principle of Priority [Art. 23.2], its application is moderated as follows:

23.9.1. prevailing usage must be maintained when the following conditions are both met:

23.9.1.1. the senior synonym or homonym has not been used as a valid name after 1899 , and

23.9.1.2. the junior synonym or homonym has been used for a particular taxon, as its presumed valid name, in at least 25 works, published by at least 10 authors in the immediately preceding 50 years and encompassing a span of not less than 10 years.

$<<$ Use of Art. 23.9.1.1 is straightforward, but Art. 23.9.1.2 presents some problems with family-group names that appear rarely in publications. If I have evidence that the name of a family-group taxon is a long-accepted name in its accustomed meaning, I add: Name in prevailing recent practice behind 
the family-group name regardless of whether it met the criteria of " 25 works, published by at least 10 authors in the immediately preceding 50 years and encompassing a span of not less than 10 years". >>

23.9.2. An author who discovers that both the conditions of 23.9.1 are met should cite the two names together and state explicitly that the younger name is valid, and that the action is taken in accordance with this article; at the same time the author must give evidence that the conditions of Art. 23.9.1.2 are met, and also state that, to his or her knowledge, the condition in Art. 23.9.1.1 applies. From the date of publication of that act the younger name has precedence over the older name. When cited, the younger but valid name may be qualified by the term 'nomen protectum' and the invalid, but older, name by the term 'nomen oblitum'. In the case of subjective synonymy, whenever the names are not regarded as synonyms the older name may be used as valid.

23.9.3. If the conditions of 23.9.1 are not met but nevertheless an author considers that the use of the older synonym or homonym would threaten stability or universality or cause confusion, and so wishes to maintain use of the younger synonym or homonym, he or she must refer the matter to the Commission for a ruling under the plenary power [Art. 81]. While the case is under consideration use of the junior name is to be maintained [Art. 82].

23.9.4. If the case is one of homonymy in family-group names resulting from similarity but not identity in the names of type-genera, see Art. 55.3.

\section{Article 24. Precedence between simultaneously published names, spellings or acts}

24.1. Automatic determination of precedence of names. When homonyms or synonyms are established simultaneously, but proposed at different ranks, in the family group, genus group or species group the name proposed at higher rank takes precedence [Arts 55.5, 56.3 and 57.7].

\section{Article 29. Family-group names}

29.1. Formation of family-group names. A family-group name is formed by adding to the stem of the name [Art. 29.3] of the type genus, or to the entire name of the type genus [see Art. 29.6], a suffix as specified in Art. 29.2.

29.2. Suffixes for family-group names. The suffix -OIDEA is used for a superfamily name, -IDEA for a family name, -INAE for a subfamily name, -INI for the name of a tribe, and -INA for the name of a subtribe. These suffixes must not be used at other family-group ranks. The suffixes of names for taxa at other ranks in the family-group are not regulated.

29.2.1. Names in the genus and species groups which have endings identical with those of the suffixes of family-group names are not affected by this article.

29.3. Determination of stem in names of type genera. The stem of a family-group name is based on the name of its type genus [Art. 63] and determined as follows.

29.3.1. If a generic name is or ends in a Greek or Latin word, or ends in a Greek or Latin suffix, the stem for the purposes of the Code is found by deleting the case ending of the appropriate genitive singular.

29.3.1.1. If the stem so formed ends in -id, those letters may be elided before adding the family-group suffixes. If, however, the unelided form is in prevailing usage, that spelling is to be maintained, whether or not it is the original spelling. 
29.3.2. If the name of a genus is or ends in a Greek word latinized with a change in ending, the stem is that appropriate to the latinized form, as determined in Art. 29.3.1.

29.3.3. If a generic name is or ends in a word not Greek or Latin, or is an arbitrary combination of letters, the stem for the purposes of the Code is that adopted by the author who establishes the new family-group taxon, either the entire generic name (see Art. 29.6), or the entire generic name with the ending elided, or the entire generic name with one or more appropriate linking letters incorporated in order to form a more euphonious family-group name.

$<<$ In some cases, the stem of the type genus is not the correct grammatical stem, but is the genitive without its case-ending. See Steyskal (1980) for some guidance in forming the stem. Also the extract from the $3^{\text {rd }}$ edition of the Code on the ICZN website (http://iczn.org/content/formation-names; accessed 1 Apr. 2014) can be of some help. Sheiko (2013) also lists many stems.

I add the remark: [changed to ... by \{author year:page number\}] to present a different spelling by an author (changed by mistake or insight or dislike?), or [corrected to ... by \{author year:page number\}] to present a possibly intentional different spelling by an author [a bit arbitrarily], or [emended to ... by \{author year:page number\}] in the strict sense of Art. 33.2.1 of the Code; i.e., an explicit statement of intentional change of spelling with citation of both spellings. I tried to establish the stem in prevailing recent practice.

As nomenclature is based on the examination of individual cases, one cannot extend a certain stem to all such generic name endings (for instance, although the correct stem of a type genus ending in -ichthys is -ichthy-, a different stem can be found in the accepted family-names).>>

29.4. Acceptance of originally formed stem. If after 1999 a new family-group name is based on a generic name which is or ends in a Greek or Latin word or ends in a Greek or Latin suffix, but its derivation does not follow the grammatical procedures of Arts 29.3.1 or 29.3.2, its original spelling must be maintained as the correct original spelling, provided

29.4.1. it has a correctly formed suffix [Art. 29.2], and

29.4.2. its stem is formed from the name of the type genus as though it were an arbitrary combination of letters [Art. 29.3.3].

29.5. Maintenance of current spellings. If a spelling of a family-group name was not formed in accordance with Art. 29.3 but is in prevailing usage, that spelling is to be maintained, whether or not it is the original spelling and whether or not its derivation from the name of the type genus is in accordance with the grammatical procedures in Arts 29.3.1 and 29.3.2.

$<<$ The central issue of Arts 29.3 and 29.5 is whether the emendation of a family-group name is justified. Today a growing number of zoologists (perhaps a majority) is against changing the originally proposed stem. Art. 29.5 shifts the balance between the two principles of zoological nomenclature: original intent/ linguistic correction vs stability, towards stability. I applied Art. 29.5 with the working principle of prevailing recent practice. $>>$

29.6. Avoidance of homonymy in family-group names. An author wishing to establish a new familygroup name must avoid its homonymy with any known previously established names by forming an appropriate stem from the name of the type genus. (See Art. 55.3.1 for the elimination of homonymy between existing family-group names). 


\section{Article 32. Original spellings}

32.5.3. A family-group name is an incorrect original spelling and must be corrected if it

32.5.3.1. has an incorrectly formed suffix [Art. 29.2], or

32.5.3.2. is formed from an unjustified emendation of a generic name (unless the unjustified emendation has become a substitute name), or

32.5.3.3. is formed from an incorrect subsequent spelling of a generic name [Art. 35.4.1], or

32.5.3.4. is formed from one of two or more original spellings of a genus-group name which was not that selected by the First Reviser [Art. 24.2.3].

$<<$ I place the correction of an obvious spelling error (type-setting error) in the family-group name in brackets immediately behind the name; e.g., $\dagger$ Jekelotodontidae [ $\dagger$ Jaekelotodontidae] Glickman 1964a: 11 (family) †Jaekelotodus Menner 1928.

If an incorrect spelling of the type genus was used, I add after the type genus the remark: [type genus as \{used incorrect spelling of the type genus\}, name must be corrected Art. 32.5.3 to ...]; e.g., $\uparrow$ Acanthoëssidae Hay 1902: 273 (family) †Acanthoessus Agassiz 1832 [type genus as $\uparrow$ Acanthoëssus, name must be corrected Art. 32.5.3 to $\uparrow$ Acanthoessidae]>>

\section{Article 34. Mandatory changes in spelling consequent upon changes in rank or combination}

34.1. Family-group names. The suffix of a family-group name must be changed when the taxon denoted by the name is raised or lowered in rank; the author and date of the name remain unchanged [Arts 23.3.1, 29.2, 50.3.1].

\section{Article 35 . The family group}

35.1. Definition. The family group encompasses all nominal taxa at the ranks of superfamily, family, subfamily, tribe, subtribe, and any other rank below superfamily and above genus that may be desired (see also Art. 10.3 for collective groups and ichnotaxa).

$<<$ Most early English and American writers used the family-group name group (and subgroup) for a grouping of some genera (equivalent to the currently used term tribe/subtribe). The word Gruppe of early writers (e.g., Bassani, Jaekel, Wagner) is also more or less equivalent to the currently used term tribe. I list the family-group names when they were used for grouping genera below the family. I did not list the (non-regulated) higher rank names of the order/class-series.>>

35.2. Provisions applicable to all family-group nominal taxa and their names. Family-group nominal taxa and their names are subject to the same provisions whatever their rank, except in respect of their suffixes [Art. 29.2] (for the application of the Principle of Coordination to family-group names, see Art. 36).

35.3. Application of family-group names. The application of each family-group name is determined by reference to the type genus of the nominal taxon [Arts 61-65].

35.4. Formation and treatment of family-group names. A family-group name is to be formed and treated in accordance with Art. 11.7 and the relevant provisions of Arts 25-34. 
35.4.1. A family-group name based upon an unjustified emendation (but see Art. 35.4.2) or an incorrect spelling of the name of the type genus must be corrected, unless it is preserved under Art. 29.5 or unless the spelling of the genus-group name used to form the family-group name is preserved under Arts 33.2.3.1 or 33.3.1.

35.4.2. If an unjustified emendation of the name of the type genus becomes its substitute name, the family-group name is then to be based on it by correcting the name to the spelling formed from the stem of the substitute name, or the whole substitute name [Art. 29.1]; the author and date of the family-group name remain unchanged.

35.5. Precedence for names in use at higher rank. If after 1999 when a name in use for a family-group taxon (e.g. for a subfamily) is found to be older than a name in prevailing usage for a taxon at higher rank in the same family-group taxon (e.g. for the family within which the older name is the name of a subfamily) the older name does not displace the younger name.

$<<$ If I found an older subfamily name, I add after the family name: Name in prevailing recent practice, Art. 35.5. If I found an older family name and if I have evidence that the current family name (or the current spelling) is in prevailing recent practice, I add: Name (or spelling) in prevailing recent practice.

I checked the family-group names against Nelson et al. (2016) and recent literature. If I use the same name and spelling as in most of these recent works, I add: Name in prevailing recent practice, or if I use the same spelling, I add: Spelling in prevailing recent practice where appropriate, or I use a combination of both phrases.>>

\section{Article 36. Principle of Coordination}

36.1. Statement of the Principle of Coordination applied to family-group names. A name established for a taxon at any rank in the family group is deemed to have been simultaneously established for nominal taxa at all other ranks in the family group; all these taxa have the same type genus, and their names are formed from the stem of the name of the type genus [Art. 29.3] with appropriate change of suffix [Art. 34.1]. The name has the same authorship and date at every rank.

36.2. Type genus. When a nominal taxon is raised or lowered in rank in the family group its type genus remains the same [Art. 61.2.2].

\section{Article 37. Nominotypical taxa}

37.1. Definition. When a family-group taxon is subdivided, the subordinate taxon that contains the type genus of the superior taxon is denoted by the same name (except for suffix) with the same author and date [Art. 36.1]; this subordinate taxon is termed the "nominotypical taxon".

37.2. Effect of change of name on nominotypical taxa. If the name in use for a family-group taxon is unavailable or invalid it must be replaced by the name valid under Art. 23.3.5; any subordinate taxa containing the type genus of the substitute nominal taxon (and therefore denoted by the valid familygroup name, with appropriate suffixes) become nominotypical taxa.

\section{Article 39. Invalidity due to homonymy or suppression of the name of the type genus}

The name of a family-group taxon is invalid if the name of its type genus is a junior homonym or has been totally or partially suppressed (see Arts 81.2.1 and 81.2.2) by the Commission. If that family-group 
name is in use it must be replaced either by the next oldest available name from among its synonyms [Art. 23.3.5], including the names of its subordinate family-group taxa, or, if there is no such synonym, by a new name based on the valid name (whether a synonym or a new replacement name (nomen novum)) of the former type genus.

$<<$ If a family-group taxon is based on a junior homonym or on a suppressed generic name, I add the remark: [invalid, Art. 39].>>

\section{Article 40 . Synonymy of the type genus}

40.1. Validity of family-group names not affected. When the name of a type genus of a nominal familygroup taxon is considered to be a junior synonym of the name of another nominal genus, the familygroup name is not to be replaced on that account alone.

40.2. Names replaced before 1961. If, however, a family-group name was replaced before 1961 because of the synonymy of the type genus, the substitute name is to be maintained if it is in prevailing usage.

40.2.1. A name maintained by virtue of this article retains its own author but takes the priority of the replaced name, of which it is deemed to be the senior synonym.

Recommendation 40A. Citation of author and date. If the author and date are cited, a family-group name maintained under the provisions of Art. 40.2.1 should be cited with its original author and date (see Recommendation 22A.2.2), followed by the date of its priority as determined by this article; the date of priority should be enclosed in parentheses.

$<<$ The changing of the rules over the century (particularly if the type genus was found to be a junior synonym) was responsible for many new family-group names. Some came into prevailing practice, others disappeared. If I have found evidence that a name replaced before 1961 is in prevailing practice, I add the remark: (\{date of priority\}) Name in prevailing recent practice, Art. 40.2. $>>$

\section{Article 53. Definitions of homonymy in the family group, genus group and species group}

53.1. Homonyms in the family group. In the family group, two or more available names having the same spelling or differing only in suffix [Art. 29.2] and denoting different nominal taxa are homonyms.

\section{Article 55. Homonymy in family-group names}

55.1. Application of the Principle of Homonymy. The Principle of Homonymy applies to all familygroup names, including names of ichnotaxa at the family-group level.

55.2. Homonymy from identical generic names. See Art. 39.

55.3. Homonymy from similar generic names. Homonymy between family-group names may result from similarity but not identity of the names of their type genera.

55.3.1. Such a case involving family-group names must be referred to the Commission for a ruling to remove homonymy unless the senior homonym is a nomen oblitum.

55.3.1.1. When the senior homonym is determined to be a nomen oblitum under the conditions of Art. 23.9.2, a new family-group name (a nomen novum) based on the same type genus may be proposed, but choosing a new stem from the name of the type genus which avoids the homonymy [Arts 29.1, 29.4 and 29.6]. 
55.4. One-letter difference. Even if the difference between two family-group names is only one letter, they are not homonyms.

55.5. Precedence of names at higher rank. Of two homonymous family-group names of identical date but established at different ranks, the one established at the higher rank is deemed to be the senior homonym [Art. 24.1].

$<<$ I note the cases of homonymy as: [preoccupied by $\{$ family-group name $\}$ in $\{\ldots\}$; not to be used, Art. 55.3].>>

\section{Article 61.3. Name-bearing types and synonymy}

61.3.2. If two or more objectively synonymous generic names have been used as the basis for names in the family group, the family-group names are objective synonyms.

$<<$ If two objectively synonymous generic names have been used as the basis for family names, I add: [junior objective synonym of \{family-group name author year\}, invalid, Art. 61.3.2] or [senior objective synonym of \{family-group name author year\}]; e.g., †Acanthoëssidae Hay 1902: 273 (family) $\uparrow$ Acanthoessus Agassiz 1832 [type genus as $\uparrow$ Acanthoëssus, name must be corrected Art. 32.5.3 to $\uparrow$ Acanthoessidae; junior objective synonym of $\uparrow$ Acanthodidae Agassiz 1844, invalid, Art. 61.3.2]. $>>$

\section{How to use the family-group names list}

A researcher wishing to create a new family-group name can use this list in the following way:

First determine the desired level, such as subtribe, tribe, subfamily or family.

Check to see if any of the genera (and their synonyms) to be included in the new taxon are in the family-group names list (if there are Recent fishes classified in the family-group name, check Van der Laan et al. 2014 and the cumulative addenda to this publication on http://www.calacademy.org/scientists/catalog-of-fishes-family-group-names/).

If none is in the list, then a new name can be proposed according to the Code. State the new family-group name, indicate the name as NEW, give a diagnosis of the new taxon and state the (valid) type genus. Use the dagger notation $(\dagger)$ correctly.

If one genus to be included in the new taxon is in the list of family-group names, check the original reference and transfer the name to the desired rank with the correct suffix. Do not change the stem of the family-group name to prevent confusion.

If two or more genera to be included in the new taxon are in the list of family-group names, check the original references, take the oldest name (to satisfy the rule of priority) and transfer the name to the desired rank with the correct suffix.

Check if the stem to be used has been used in a family-group name for any animal, not only fishes, to avoid homonymy. This can be done efficiently by searching for the stem on Google.

Mention the to be created family-group name in the abstract and in the keywords of the new publication. 


\section{Family-group names of fossil fishes}

No valid family-group name description could be located for the following family-group names currently in usage:

$\uparrow$ Brindabellaspidae, †Diabolepididae, †Dorsetichthyidae, †Erichalcidae, †Holodipteridae, $\dagger$ Kentuckiidae, $†$ Lepidaspididae, $†$ Loganelliidae and $\uparrow$ Pituriaspididae.

Family-group names not anymore in 'Fishes'

$\uparrow$ Angustidontidae Cooper 1936: 93 (family) $\uparrow$ Angustidontus Cooper 1936 [jaws of a shrimp]

$\uparrow$ Pseudodontichthyidae Obruchev 1964c: 255 (family) †Pseudodontichthys Skeels 1962 [a phyllocarid crustacean, Andrews et al. 1967: 675]

\section{Family-group names with unknown systematic placement}

$\uparrow$ Propenserinae Applegate 1970: 398 (subfamily) †Propenser Applegate 1970 [fossilized portions of a coelacanth and a pycnodont]

$\dagger$ Palaeodontidae Obruchev 1964a: 43 (family) $†$ Palaeodus Rohon 1890

$\uparrow$ Rogeniidae Jordan 1919: 8 (family) †Rogenio Jordan 1907 [family name sometimes seen as $\uparrow$ Rogenionidae]

\section{CHORDATA}

SUBPHYLUM CRANIATA

Order $\uparrow$ Myllokunmingiiformes

Family $\uparrow$ Myllokunmingiidae Shu 2003

$\uparrow$ Myllokunmingiidae Shu 2003: 727 (family) †Myllokunmingia Shu, Zhang \& Han 1999

\section{INFRAPHYLUM MYXINOMORPHI}

\section{CLASS MYXINI}

Order Myxiniformes

Family Myxinidae Rafinesque 1815

\section{INFRAPHYLUM VERTEBRATA}

CLASS PETROMYZONTIDA (CEPHALASPIDOMORPHI)

$\dagger$ Gilpichthyidae Halstead 1993: 580 (family) †Gilpichthys Bardack \& Richardson 1977 [name only, published after 1960, not available, Art. 13.1.1]

$\uparrow$ Pipisciidae Halstead 1993: 580 (family) $†$ Pipiscius Bardack \& Richardson 1977 [name only, published after 1960, not available, Art. 13.1.1]

\section{Order Petromyzontiformes}

$\uparrow$ Hardistiellidae Halstead 1993: 580 (family) $\uparrow$ Hardistiella Janvier \& Lund 1983 [name only, published after 1960, not available, Art. 13.1.1]

Family $\uparrow$ Mayomyzontidae Bardack \& Zangerl 1971

$\uparrow$ Mayomyzontidae Bardack \& Zangerl 1971: 80 (family) †Mayomyzon Bardack \& Zangerl 1968

[family name sometimes seen as $\uparrow$ Mayomyzonidae]

Family Petromyzontidae Bonaparte 1831

Family Geotriidae Gill 1893

Family Mordaciidae Gill 1893

SUPERCLASS $\uparrow$ PTERASPIDOMORPHI

CLASS $\uparrow$ PTERASPIDOMORPHA

Subclass $\uparrow$ ASTRASPIDA 


\section{Order $\uparrow$ Astraspidiformes}

Family $\uparrow$ Astraspididae Eastman 1917, spelling in prevailing recent practice

$\uparrow$ Astraspidae Eastman 1917: 237 (family) †Astraspis Walcott 1892 [family name also seen as $\dagger$ Astraspididae]

Family †Oniscolepididae Märss \& Karatajūtè-Talimaa 2009

†Oniscolepididae Märss \& Karatajūtė-Talimaa 2009: 45 (family) †Oniscolepis Pander 1856

Family $\uparrow$ Tesakoviaspididae Karatajūtė-Talimaa \& Smith 2004

$\dagger$ Tesakoviaspididae Karatajūtè-Talimaa \& Smith 2004: 56 (family) †Tesakoviaspis KaratajūtèTalimaa \& Smith 2004

Family $\uparrow$ Eriptychiidae Tarlo 1962

$\dagger$ Eriptychiidae Tarlo 1962: 254 (family) †Eriptychius Walcott 1892

\section{Subclass $†$ ARANDASPIDA}

$\uparrow$ Aseraspididae Halstead 1993: 573 (family) †Aseraspis Dineley \& Loeffler 1976 [name only, published after 1960, not available, Art. 13.1.1]

\section{Order $\uparrow$ Arandaspidiformes}

Family $\uparrow$ Arandaspididae Ritchie \& Gilbert-Tomlinson 1977

$\uparrow$ Arandaspididae Ritchie \& Gilbert-Tomlinson 1977: 351 (family) †Arandaspis Ritchie \& Gilbert-Tomlinson 1977

$\uparrow$ Porophoraspidae Halstead 1993: 573 (family) †Porophoraspis Ritchie and Gilbert-Tomlinson 1977 [name only, published after 1960, not available, Art. 13.1.1]

\section{Subclass $†$ HETEROSTRACI}

Family $\uparrow$ Ariaspididae Elliott \& Swift 2010

$\uparrow$ Ariaspididae Elliott \& Swift 2010: 1874 (family) †Ariaspis Denison 1963

\section{Order $\uparrow$ Cardipeltiformes}

Family $\uparrow$ Cardipeltidae Bryant 1933

$\uparrow$ Cardipeltidae Bryant 1933: 307 (family) $†$ Cardipeltis Branson \& Mehl 1931 [family name sometimes seen as $\uparrow$ Cardipeltididae]

\section{Order $\uparrow$ Corvaspidiformes}

Family $\uparrow$ Corvaspididae Dineley 1955, spelling in prevailing recent practice

$\dagger$ Corvaspidae Dineley 1955: 178 (family) †Corvaspis Woodward 1934 [family name also seen as $\uparrow$ Corvaspididae]

\section{Order $\uparrow$ Lepidaspidiformes}

Family $\uparrow$ Lepidaspididae

$\dagger$ Lepidaspididae Halstead 1993: 573 (family) †Lepidaspis Dineley \& Loeffler 1976 [name only, published after 1960, not available, Art. 13.1.1; family name sometimes seen as $\dagger$ Lepidaspidae]

\section{Order $\uparrow$ Tesseraspidiformes}

Family $\uparrow$ Tesseraspididae Berg 1955, spelling in prevailing recent practice

$\dagger$ Tesseraspidae Berg 1955: 25 (family) †Tesseraspis Wills 1935 [family name also seen as $\dagger$ Tesseraspididae]

$\uparrow$ Natlaspididae Halstead 1993: 575 (family) $\uparrow$ Natlaspis Dineley \& Loeffler, 1976 [name only, published after 1960, not available, Art. 13.1.1]

\section{Order $\dagger$ Traquairaspidiformes}

Family $\uparrow$ Traquairaspididae Kiaer 1932, spelling in prevailing recent practice 
$\dagger$ Traquairaspidae Kiaer 1932: 25 (family) †Traquairaspis Kiaer 1932 [family name also seen as $\dagger$ Traquairaspididae]

$\dagger$ Weigeltaspiden Brotzen 1933: 655 (family) †Weigeltaspis Brotzen 1933 [published not in latinized form after 1899, not available]

$\uparrow$ Weigeltaspidae Berg 1940: 361 [family name sometimes seen as $†$ Weigeltaspididae; could date to Obruchev 1937 from an article in Трансактионс оф тхе Палеонтологикал институте [Transactions of the Paleontological Institute], not located yet]

$\dagger$ Phialaspididae White 1946b: 236 (family) †Phialaspis Wills 1935 [family name sometimes seen as $†$ Phialaspidae]

\section{Order $\uparrow$ Tolypelepidiformes}

Family $\uparrow$ Tolypelepididae Strand 1934 (1932), name and spelling in prevailing recent practice, Art. 40.2

$\dagger$ Tolypaspidae Kiaer 1932: 24 (family) †Tolypaspis Schmidt 1893 [senior objective synonym of $\uparrow$ Tolypelepidae; family name sometimes seen as $\uparrow$ Tolypaspididae]

$\uparrow$ Tolypelepidae Strand 1934: 328 (family) †Tolypelepis Pander 1856 [junior objective synonym of †Tolypaspidae, invalid, Art. 61.3.2, but name in prevailing recent practice Art. 40.2; family name also seen as $\uparrow$ Tolypelepididae]

\section{Order $\uparrow$ Cyathaspidiformes}

\section{Suborder $\uparrow$ Amphiaspidoidei}

Family $\uparrow$ Amphiaspididae Obruchev 1939

$\uparrow$ Amphiaspididae Obruchev 1939: 327 (family) †Amphiaspis Obruchev 1936 [family name sometimes seen as †Amphiaspidae]

Family $\uparrow$ Gabreyaspididae Novitskaya 1968

$\dagger$ Gabreyaspididae Novitskaya 1968: 49 (family) †Gabreyaspis Novitskaya 1968

Family †Olbiaspididae Obruchev 1964

$\dagger$ Olbiaspididae Obruchev 1964a: 78 (family) †Olbiaspis Obruchev 1964 [family name sometimes seen as $\dagger$ Obliaspidae]

$\uparrow$ Angaraspididae Halstead 1993: 576 (family) †Angaraspis Obruchev 1964 [name only, published after 1960, not available, Art. 13.1.1]

Family $†$ Edaphaspididae Novitskaya 1968

$\dagger$ Edaphaspididae Novitskaya 1968: 57 (family) †Edaphaspis Novitskaya 1968

\section{Suborder $\uparrow$ Hibernaspidoidei}

Family $\uparrow$ Eglonaspididae Tarlo 1962

$†$ Eglonaspididae Tarlo 1962: 268 (family) †Eglonaspis Obruchev 1959

Family $\uparrow$ Hibernaspididae Obruchev 1939

$†$ Hibernaspididae Obruchev 1939: 320 (family) †Hibernaspis Obruchev 1939

Family $\uparrow$ Aphataspididae Novitskaya 1971

$\uparrow$ Aphataspididae Novitskaya 1971: 104 (family) $\uparrow$ Aphataspis Obruchev 1964

\section{Suborder $\uparrow$ Siberiaspidoidei}

Family $\uparrow$ Siberiaspididae Novitskaya 1968

$\dagger$ Siberiaspididae Novitskaya 1968: 59, 60 (family) †Siberiaspis Obruchev 1964

$\uparrow$ Tuxeraspididae Novitskaya 1968: 59 (family) †Tuxeraspis Novitskaya 1971 [no valid type genus, not available, Art. 11.7.1.1; also name only]

$†$ Tuxeraspididae Novitskaya 1971: 43 (family) †Tuxeraspis Novitskaya 1971

\section{Suborder $\uparrow$ Cyathaspidoidei}

Family $\uparrow$ Cyathaspididae Kiaer 1932, name and spelling in prevailing recent practice

$†$ Poraspidae Kiaer 1932: 12 (family) †Poraspis Kiaer 1930 [family name sometimes seen as $\dagger$ Poraspididae or as subfamily $\dagger$ Poraspidinae] 
$\dagger$ Dinaspidae Kiaer 1932: 15 (family) $†$ Dinaspis Kiaer 1932 [type genus preoccupied by Dinaspis Leonardi 1911 in Hemiptera; invalid, Art. 39; family name sometimes seen as $\uparrow$ Dinaspididae] $\uparrow$ Palaeaspidae Kiaer 1932: 15 (family) †Palaeaspis Claypole 1885 [type genus preoccupied by $\uparrow$ Palaeaspis Gray 1870 in Reptilia; invalid, Art. 39; family name sometimes seen as $\uparrow$ Palaeaspididae]

$\uparrow$ Anglaspidae Kiaer 1932: 19 (family) †Anglaspis Jaekel 1927 [family name sometimes seen as $\uparrow$ Anglaspididae]

$\dagger$ Cyathaspidae Kiaer 1932: 21 (family) †Cyathaspis Lankester 1865 [family name also seen as $\dagger$ Cyathaspididae]

$\uparrow$ Diplaspidae Kiaer 1932: 25 (family) †Diplaspis Matthew 1887 [family name sometimes seen as $\uparrow$ Diplaspididae]

$\dagger$ Dinaspidellidae Strand 1934: 327 (family) †Dinaspidella Strand 1934

$\dagger$ Irregularaspidae [†Irregulareaspidae] Kiaer \& Heinz 1935: 42 (family) †Irregulareaspis Zych 1931 [family name sometimes seen as $\dagger$ Irregulareaspididae]

$\uparrow$ Dictyonaspidae [†Dictyaspidae] Berg 1940: 362 (family) †"Dictyonaspis" (? †Dictyaspis Kiaer 1932) [no valid type genus, not available, Art. 11.7.1.1; probably a printing error for $\uparrow$ Dictyaspidae, also Berg 1955: 27; type genus preoccupied by Dictyaspis Haeckel 1887 in Radiozoa; invalid, Art. 39; family name corrected to $\uparrow$ Dictyaspididae by Schultz 1948: 221]

$\uparrow$ Americaspidae Fowler 1947: 3 (family) †Americaspis White \& Moy-Thomas 1941 [family name sometimes seen as $\uparrow$ Americaspididae]

$\uparrow$ Dictyaspidellidae Fowler 1947: 4 (family) †Dictyaspidella Strand 1934

$\uparrow$ Torpedaspididae Halstead 1993: 574 (family) †Torpedaspis Broad \& Dineley 1973 [name only, published after 1960, not available, Art. 13.1.1]

$†$ Boothiaspidinae Elliott 2016: 1213 (subfamily) $†$ Boothiaspis Broad, 1973

Family $\uparrow$ Ctenaspididae Kiaer 1930, spelling in prevailing recent practice

$\dagger$ Ctenaspidae Kiaer 1930: 5 (family) †Ctenaspis Kiaer 1930 [family name also seen as $\dagger$ Ctenaspididae]

\section{Order $\uparrow$ Pteraspidiformes}

Incertae sedis:

$\dagger$ Belgicaspinae Zych 1931: 86 (subfamily) †Belgicaspis Zych 1931

$\dagger$ Larnovaspididae Novitskaya 1986: 91 (family) †Larnovaspis Blieck 1984 [variously credited to Holstead or Halstead, but Novitskaya is responsible for the description and can be credited as author] $\uparrow$ Penygaspididae Halstead 1993: 575 (family) †Penygaspis Stensiö 1958 [name only, published after 1960, not available, Art. 13.1.1]

$\uparrow$ Podolaspinae Zych 1931: 86 (subfamily) $\uparrow$ Podolaspis Zych 1931 [also as a new family, †Podolaspididae, in Novitskaya 1983: 161]

$\dagger$ Protaspidae Bryant 1933: 287 (family) †Protaspis Bryant 1933 [family name sometimes seen as $†$ Protaspididae]

$\uparrow$ Psephaspididae Novitskaya 1983: 165 (family) †Psephaspis Ørvig 1961 [name only, published after 1960, not available, Art. 13.1.1]

$\dagger$ Pteraspididae Claypole 1885: 56 (family) †Pteraspis Kner 1847 [type genus inferred from the stem,

Art. 11.7.1.1; family name sometimes seen as †Pteraspidae]

$†$ Rostraspinae Zych 1931: 86 (subfamily) ? [no valid type genus, not available, Art. 11.7.1.1]

$\dagger$ †kalviaspididae Karatajūtè-Talimaa 1989: 79 (family) $†$ Skalviaspis Karatajūtè-Talimaa 1989

\section{Suborder $\uparrow$ Pteraspidoidei}

Family $\uparrow$ Anchipteraspididae Elliott 1984

$\uparrow$ Anchipteraspidinae Elliott 1984: 173 (subfamily) †Anchipteraspis Elliott 1984

Family $\uparrow$ Doryaspididae Heintz 1962 
$\uparrow$ Doryaspididae Heintz in Tarlo 1962: 265 (family) †Doryaspis White 1935

$\dagger$ Lyktaspididae Heintz 1968: 79 (family) †Lyktaspis Heintz 1968

Family $\uparrow$ Protopteraspididae Novitskaya 1983

$\uparrow$ Protopteraspididae Novitskaya 1983: 161 (family) †Protopteraspis Leriche 1924

†Gigantaspididae Pernègre \& Elliott 2008: 397 (family) †Gigantaspis Heintz 1962

Family $\uparrow$ Rhinopteraspididae Novitskaya 1983

$\uparrow$ Rhinopteraspiden Brotzen 1933: 655 (family) $†$ Rhinopteraspis Jaekel 1919 [published not in latinized form after 1899, not available]

\section{Suborder $\uparrow$ Psammosteoidei}

$\dagger$ Rhinopteraspididae Novitskaya 1983: 164 (family) †Rhinopteraspis Jaekel 1919

Family $\uparrow$ Drepanaspididae Traquair 1900 , spelling in prevailing recent practice

$\uparrow$ Drepanaspidae Traquair 1900: 844 (family) †Drepanaspis Schlüter 1887 [family name also seen as $\uparrow$ Drepanaspididae]

Family $\uparrow$ Guerichosteidae Tarlo 1965

†Guerichosteidae Tarlo 1965: 113 (family) †Guerichosteus Tarlo 1965

Family $\uparrow$ Obrucheviidae Tarlo 1965 (1955), name in prevailing recent practice, Art. 40.2

$\uparrow$ Aspidosteidae Berg 1955: 23 (family) $\uparrow$ Aspidosteus Obruchev 1941 [senior objective synonym of $\dagger$ Obrucheviidae Tarlo 1965]

†Obrucheviidae Tarlo 1965: 117 (family) †Obruchevia Whitley 1940 [junior objective synonym of $\uparrow$ Aspidosteidae, invalid, Art. 61.3.2, but name in prevailing recent practice Art. 40.2]

Family $\uparrow$ Pycnosteidae Tarlo 1962

$\uparrow$ Pycnosteidae Tarlo 1962: 261 (family) †Pycnosteus Preobrazhensky 1911 [type genus author also seen as Preobrajensky or Preobrashensky]

Family †Psammolepididae Tarlo 1962

$\uparrow$ Psammolepididae Tarlo 1962: 261 (family) $\uparrow$ Psammolepis Agassiz 1844

Family $\uparrow$ Psammosteidae Traquair 1896

$\dagger$ Psammosteidae Traquair 1896: 260 (family) $†$ Psammosteus Agassiz 1845

$\uparrow$ Placosteinae Glinskiy 2018: 231 (subfamily) †Placosteus Agassiz 1844

\section{SUPERCLASS †ANASPIDOMORPHI}

\section{Order $\dagger$ Jamoytiiformes}

Family $\uparrow$ Jamoytiidae White 1946

$\dagger$ Jamoytiidae White 1946a: 96 (family) †Jamoytius White 1946

Family †Achanarellidae Newman 2002

$\uparrow$ Achanarellidae Newman 2002: 934 (family) †Achanarella Newman 2002

Family $\uparrow$ Euphaneropidae Woodward 1900

$\dagger$ Euphaneropidae Woodward 1900: 418 (family) †Euphanerops Woodward 1900 [family name sometimes seen as $†$ Euphaneropsidae or $\uparrow$ Euphaneropididae]

$\dagger$ Lasaniidae Goodrich 1909: 202 (family) †Lasanius Traquair 1898

\section{CLASS †ANASPIDA}

\section{Order $\uparrow$ Endeiolepidiformes}

Family $\uparrow$ Endeiolepididae Stensiö 1939, spelling in prevailing recent practice

$\dagger$ Endeiolepidae Stensiö 1939: 18 (family) †Endeiolepis Stensiö 1939 [family name also seen as $\dagger$ Endeiolepididae]

\section{Order $\uparrow$ Anaspidiformes}

Family $\uparrow$ Birkeniidae Traquair 1900

$\uparrow$ Birkeniidae Traquair 1900: 837 (family) $†$ Birkenia Traquair 1898

Family $\uparrow$ Pterygolepididae Obruchev 1964 (1947), name and spelling in prevailing recent practice, Art. 40.2 
$\dagger$ Pterolepidae Abel 1919: ix (family) †Pterolepis Kiaer 1911 [type genus preoccupied by Pterolepis Rambur 1838 in Orthoptera; invalid, Art. 39; family name sometimes seen as $\dagger$ Pterolepididae]

$\uparrow$ Pterolepidopidae Fowler 1947: 5 (family) †Pterolepidops Fowler 1947 [senior objective synonym of $\uparrow$ Pterygolepididae Obruchev 1964]

$\uparrow$ Pterygolepididae Obruchev 1964a: 112 (family) †Pterygolepis Cossmann 1920 [junior objective synonym of $\uparrow$ Pterolepidopidae, but name in prevailing recent practice Art. 40.2; family name also seen as $\uparrow$ Pterygolepidae]

Family $\uparrow$ Rhyncholepididae Kiaer 1924, spelling in prevailing recent practice

$\uparrow$ Rhyncholepidae Kiaer 1924: 129 (family) $\uparrow$ Rhyncholepis Kiaer 1911 [family name also seen as $\uparrow$ Rhyncholepididae]

Family $\uparrow$ Pharyngolepididae Kiaer 1924, spelling in prevailing recent practice

$†$ Pharyngolepidae Kiaer 1924: 62 (family) †Pharyngolepis Kiaer 1911 [family name also seen as $†$ Pharyngolepididae]

Family $\uparrow$ Tahulalepididae Blom, Märss \& Miller 2002

$\dagger$ Tahulalepididae Blom, Märss \& Miller 2002: 305 (family) †Tahulalepis Blom, Märss \& Miller 2002

Family $\uparrow$ Septentrioniidae Blom, Märss \& Miller 2002

$\dagger$ †Septentrioniidae Blom, Märss \& Miller 2002: 307 (family) †Septentrionia Blom, Märss \& Miller 2002

Family $\uparrow$ Ramsaasalepididae Blom, Märss \& Miller 2003

$\uparrow$ Ramsaasalepididae Blom, Märss \& Miller 2003: 57 (family) †Ramsaasalepis Blom, Märss \& Miller 2003

\section{SUPERCLASS †THELODONTOMORPHI}

\section{CLASS $\uparrow$ THELODONTI}

Family $\uparrow$ Helenolepididae Wilson \& Märss 2009

$\dagger$ Helenolepididae Wilson \& Märss 2009: 306 (family) †Helenolepis Karatajūtè-Talimaa 1978

Family †Longodidae Märss 2006

$\dagger$ Longodidae Märss 2006: 59 (family) †Longodus Märss 2006 [family name sometimes seen as $\dagger$ Longodontidae]

Family †Oeseliidae Märss 2005

†Oeseliidae Märss 2005: 183 (family) †Oeselia Märss 2005

\section{Order $\uparrow$ Archipelepidiformes}

Family $\uparrow$ Archipelepididae Märss 2001

$\uparrow$ Archipelepididae Märss in Soehn, Märss, Caldwell \& Wilson 2001: 652 (family) $\uparrow$ Archipelepis Märss 2001

Family †Boothialepididae Märss 1999

†Boothialepididae Märss 1999: 1082 (family) †Boothialepis Märss 1999

\section{Order $\uparrow$ Furcacaudiformes}

Family †Furcacaudidae Wilson \& Caldwell 1998

$\dagger$ Furcacaudidae Wilson \& Caldwell 1998: 15 (family) $†$ Furcacauda Wilson \& Caldwell 1998

Family $\uparrow$ Pezopallichthyidae Wilson \& Caldwell 1998

$†$ Pezopallichthyidae Wilson \& Caldwell 1998: 12 (family) †Pezopallichthys Wilson \& Caldwell 1998

Family $\uparrow$ Nikoliviidae Karatajūtè-Talimaa 1978

$\uparrow$ Nikoliviidae Karatajūtè-Talimaa 1978: 140 (family) $\uparrow$ Nikolivia Karatajūtè-Talimaa 1978

Family $\uparrow$ Lanarkiidae Obruchev 1949 
$\dagger$ Lanarkiidae Obruchev 1949: 316 (family) †Lanarkia Traquair 1898

Family †Drepanolepididae Wilson \& Märss 2009

$\dagger$ Drepanolepididae Wilson \& Märss 2009: 306 (family) †Drepanolepis Wilson \& Caldwell 1998

Family $\uparrow$ Barlowodidae Märss, Wilson \& Thorsteinsson 2002

$\dagger$ Barlowodidae Märss, Wilson \& Thorsteinsson 2002: 113 (family) †Barlowodus Märss,

Wilson \& Thorsteinsson 2002 [family name sometimes seen as †Barlowodontidae]

Family $\uparrow$ Apalolepididae Turner 1976

$\uparrow$ Apalolepididae Turner 1976: 15 (family) $\uparrow$ Apalolepis Karatajūtė-Talimaa 1968

\section{Order $\uparrow$ Thelodontiformes}

Family $\uparrow$ Thelodontidae Jordan 1905, name in prevailing recent practice

$\uparrow$ Coelolepiden Pander 1856: 64 (family) †Coelolepis Pander 1856 [latinized to $†$ Coelolepidae by Woodward 1891a: 157 and by Berg 1940: 363; considered valid with this authorship by Woodward 1891a: 157, by Traquair 1900: 828, by Obruchev 1964a: 42, by Karatajūté-Talimaa 1968: 34 and by Halstead 1993: 576; family name sometimes seen as †Coelolepididae]

$\uparrow$ Thelodontidae Jordan 1905: 573 (family) †Thelodus Agassiz 1838 [Jordan 1923: 85 and Burrow et al. 2013: 1046 claim $\uparrow$ Thelodontidae as the correct family name; family name sometimes seen as $\uparrow$ Thelodontididae]

Family †Eestilepididae Märss, Wilson \& Thorsteinsson 2002

$\dagger$ Eestilepididae Märss, Wilson \& Thorsteinsson 2002: 112 (family) †Eestilepis Märss, Wilson \& Thorsteinsson 2002

Family $\uparrow$ Turiniidae Obruchev 1964

$\uparrow$ Turiniidae Camp \& Allison 1961: 502 (family) †Turinia Traquair 1896 [name only, published after 1960, not available, Art. 13.1.1]

$\dagger$ Turiniidae Obruchev 1964a: 43 (family) †Turinia Traquair 1896 [family name sometimes seen as $†$ Turinidae]

Family $\uparrow$ Talivaliidae Märss, Wilson \& Thorsteinsson 2002

$\dagger$ Talivaliidae Märss, Wilson \& Thorsteinsson 2002: 110 (family) †Talivalia Märss, Wilson \& Thorsteinsson 2002

\section{Order $†$ Sandiviiformes}

Family †Sandiviidae Karatajūtè-Talimaa \& Märss 2004

$\uparrow$ Sandiviinae Karatajūtè-Talimaa 1997b: 6 (subfamily) †Sandivia Karatajūtè-Talimaa 1997 [name only, published after 1960, not available, Art. 13.1.1]

$\uparrow$ Sandiviidae Karatajūtè-Talimaa \& Märss 2004: 21 (family) †Sandivia Karatajūtè-Talimaa 1997

Family $\uparrow$ Stroinolepididae Märss \& Karatajūtè-Talimaa 2004

$\uparrow$ Stroinolepididae Märss \& Karatajūtè-Talimaa in Karatajūtè-Talimaa \& Märss 2004: 22 (family) $\dagger$ Stroinolepis Märss \& Karatajūtè-Talimaa 2002

Family †Angaralepididae Märss \& Karatajūtè-Talimaa 2004

$\uparrow$ Angaralepidinae Karatajūtè-Talimaa 1997b: 14 (subfamily) †Angaralepis Karatajūtè-Talimaa 1997 [name only, published after 1960, not available, Art. 13.1.1]

†Angaralepididae Märss \& Karatajūtė-Talimaa in Karatajūtè-Talimaa \& Märss 2004: 22 (family) $\uparrow$ Angaralepis Karatajūtè-Talimaa 1997

\section{Order $\uparrow$ Loganelliiformes}

Family †Loganelliidae

$\dagger$ Loganiidae Karatajūtè-Talimaa 1978: 66 (family) $†$ Logania Gross 1967 [type genus preoccupied by Logania Distant 1884 in Lepidoptera; invalid, Art. 39]

$\nmid$ Loganellidae Halstead 1993: 576 (family) †Loganellia Fredholm 1990 [name only, published after 1960, not available, Art. 13.1.1] 
$\dagger$ Loganelliinae Karatajūtè-Talimaa 1997b: 14 (subfamily) †Loganellia Fredholm 1990 [name only, published after 1960, not available, Art. 13.1.1]

$\dagger$ Loganelliidae Märss, Wilson \& Thorsteinsson 2002: 90 (family) †Loganellia Fredholm 1990 [not published according to the rules, not available]

$\dagger$ Loganelliidae Karatajūtè-Talimaa \& Märss 2004: 23 (family) †Loganellia Fredholm 1990 [not published according to the rules, not available]

$\dagger$ Loganelliidae Nelson, Grande \& Wilson 2016: 32 (family) †Loganellia Fredholm 1990 [not published according to the rules, not available]

Family $\uparrow$ Nunavutiidae Märss, Wilson \& Thorsteinsson 2002

$\uparrow$ Nunavutiidae Märss, Wilson \& Thorsteinsson 2002: 94 (family) †Nunavutia Märss, Wilson \& Thorsteinsson 2002

\section{Order $\uparrow$ Shielliiformes}

Family †Shieliidae Märss, Wilson \& Thorsteinsson 2002

$†$ †hieliidae Märss, Wilson \& Thorsteinsson 2002: 95 (family) †Shielia Märss 1998

\section{Order $\uparrow$ Phlebolepidiformes}

Family $\uparrow$ Phlebolepididae Berg 1940, spelling in prevailing recent practice

$\dagger$ Phlebolepidae Berg 1940: 363 (family) $†$ Phlebolepis Pander 1856 [family name also seen as $\dagger$ Phlebolepididae

Family $\dagger$ Katoporodidae Märss, Wilson \& Thorsteinsson 2002

$\uparrow$ Katoporidae Karatajūtè-Talimaa 1970: 40 (family) †Katoporus Gross 1967 [type genus preoccupied by Katoporus John 1956 in Lepidoptera; invalid, Art. 39]

$\uparrow$ Katoporodidae Soehn, Märss, Caldwell \& Wilson 2001: 656 (family) †Katoporodus Turner in Turner \& Peel 1986 [not published according to the rules, not available]

$\dagger$ Katoporodidae Märss, Wilson \& Thorsteinsson 2002: 98 (family) †Katoporodus Turner \& Peel in Peel 1986 [family name sometimes seen as †Katoporididae and $\uparrow$ Katoporodontidae]

\section{SUPERCLASS †OSTEOSTRACOMORPHI \\ CLASS †OSTEOSTRACI \\ Order $\uparrow$ Cephalaspidiformes \\ Suborder $\uparrow$ Ateleaspidoidei}

Family $\uparrow$ Ateleaspididae Traquair 1900, spelling in prevailing recent practice

$\uparrow$ Ateleaspidae Traquair 1900: 834 (family) $\uparrow$ Ateleaspis Traquair 1900 [family name also seen as $\uparrow$ Ateleaspididae or $\uparrow$ Ateleasididae]

$\uparrow$ Hemicyclaspinae Heintz 1940: 98 (subfamily) $†$ Hemicyclaspis Lankester 1870 [family name also seen as $\uparrow$ Hemicyclaspididae]

$\dagger$ Hirellidae Heintz 1949: 321 (family) $\dagger$ Hirella Cossmann 1920

$\uparrow$ Aceraspidae Stensiö 1958: 216 (family) †Aceraspis Kiaer 1911 [family name sometimes seen as $\uparrow$ Aceraspididae]

Family $†$ Kalanaspididae Tinn \& Märss 2018

$†$ Kalanaspididae Tinn \& Märss 2018: [3] (family) †Kalanaspis Tinn \& Märss 2018

\section{Suborder $\uparrow$ Cephalaspidoidei}

Family $†$ Cephalaspididae Agassiz 1844, spelling in prevailing recent practice

$\uparrow$ Cephalaspides Agassiz 1844 Tome II pt. 1: 308 (family) †Cephalaspis Agassiz 1835 [family name sometimes seen as †Céphalaspides; latinized to $†$ Cephalaspidini by Bonaparte 1846: 4 (subfamily), latinized to †Cephalaspidae by Owen 1846: 50, Huxley 1861: 38 and Günther 1880: 353, latinized to $\dagger$ Cephalaspides by Giebel 1846: 64 and Giebel 1847: 261, latinized to $\uparrow$ Cephalaspida by Asmuss 1856: 10; considered valid with this authorship by Müller 1846: 122, 151, by M'Coy 1848: 6, by Bronn 1849: 656, by Quenstedt 1852: 231, by M'Coy 1855: 
598, by Pander 1856: 43, by Bleeker 1859: XI, by Denison 1951: 188 and by Halstead 1993: 578 Art. 11.7.2; not the order Cephalaspidea Fischer 1883 in Mollusca]

Family $\uparrow$ Parameteoraspididae Afanassieva 1991

$\uparrow$ Parameteoraspididae Afanassieva 1991: 79 (family) †Parameteoraspis Blieck, Goujet \& Janvier 1987

$†$ Escuminaspididae Arsenault \& Janvier 1995: 20 (family) †Escuminaspis Ørvig 1957

Family $\uparrow$ Superciliaspididae Scott \& Wilson 2014

$\uparrow$ Superciliaspididae Scott \& Wilson 2014: 169 (family) †Superciliaspis Adrain \& Wilson 1994

Suborder $\uparrow$ Scolenaspidoidei

Family $\uparrow$ Scolenaspididae Afanassieva 1991

$\dagger$ Scolenaspididae Afanassieva 1991: 91 (family) †Scolenaspis Jarvik 1954

Family $\uparrow$ Zenaspididae Stensiö 1958, spelling in prevailing recent practice

$\nmid$ Zenaspididae Stensiö 1958: 215 (family) †Zenaspis Lankester 1870 [family name also seen as

$†$ Zenaspidae]

\section{Order $\uparrow$ Thyestiformes}

Family $\uparrow$ Procephalaspididae Stensiö 1958

$†$ Procephalaspididae Stensiö 1958: 215 (family) $†$ Procephalaspis Denison 1951

Family $\uparrow$ Thyestidae Rohon 1892

$\uparrow$ Thyestidae Rohon 1892: 85 (family) †Thyestes Eichwald 1854 [Pierce in Fowler 1947: 4 and

Fowler 1965a: 179 used $\uparrow$ Thyesteidae; family name sometimes seen as $\uparrow$ Thyestiidae]

Family $\uparrow$ Witaaspididae Afanassieva 1991

†Witaaspididae Afanassieva 1991: 67 (family) †Witaaspis Robertson 1939

Family $\uparrow$ Tremataspididae Woodward 1891, spelling in prevailing recent practice

$\uparrow$ Tremataspidae Woodward 1891a: 201 (family) †Tremataspis Schmidt 1866 [family name also seen as $\uparrow$ Tremataspididae]

†Odontotodontidae Jordan 1905: 506 (family) †Odontotodus Pander 1856

†"new family" Patten 1931: 672 (family) $†$ Dartmuthia Patten 1931 [as a new family, but without the name, not available]

$\dagger$ Dartmuthiidae Robertson 1935: 282 (family) †Dartmuthia Patten 1931

†Oeselaspidae Robertson 1935: 282 (family) †Oeselaspis Robertson 1935 [family name sometimes seen as $\dagger$ Oeselaspididae or as subfamily $\dagger$ Oeselaspidinae]

$\uparrow$ Didymaspidae Berg 1940: 357 (family) $†$ Didymaspis Lankester 1867 [family name sometimes seen as $\uparrow$ Didymaspididae or as subfamily $\uparrow$ Didymaspidinae]

$\uparrow$ Sclerodidae Berg 1940: 357 (family) $\uparrow$ Sclerodus Agassiz 1839 [corrected to $\uparrow$ Sclerodontidae by Fowler 1947: 4, confirmed by Romer 1966: 347 and by Halstead 1993: 578]

$\dagger$ Stigmolepidae Fowler 1947: 4 (family) $†$ Stigmolepis Pander 1856

$\dagger$ Timanaspidinae Obruchev in Kossovoy \& Obruchev 1962: 1149 (subfamily) †Timanaspis Obruchev 1962 [English translation: 108; also as a new family, $†$ Timanaspididae, in Halstead 1993: 578]

$†$ Tannuaspididae Obruchev 1964a: 97 (family) †Tannuaspis Obruchev 1956

$\dagger$ Tyriaspididae Halstead 1993: 578 (family) †Tyriaspis Heintz 1967 [name only, published after 1960, not available, Art. 13.1.1]

$\uparrow$ Aestiaspidinae Afanassieva 1996: 567 (subfamily) †Aestiaspis Janvier \& Lelièvre 1994 [English edition: 69]

Family $\uparrow$ Kiaeraspididae Stensiö 1932, spelling in prevailing recent practice

$\uparrow$ Kiaeraspinae Stensiö 1932b: 75 (subfamily) †Kiaeraspis Stensiö 1927 [family name also seen as $\uparrow$ Kiaeraspididae] 
$\uparrow$ Nectaspididae Stensiö 1958: 220 (family) $\uparrow N e c t a s p i s$ Wängsjö 1952 [changed to $\uparrow$ Nectaspidae by Fowler 1965b: 348]

$\uparrow$ Axinaspididae Janvier 1981: 115 (family) †Axinaspis Wängsjö 1952

$\uparrow$ Acrotomaspididae Janvier 1981: 115 (family) †Acrotomaspis Wängsjö 1952 [also as new subfamily †Acrotomaspidinae]

\section{Order $\uparrow$ Benneviaspidiformes}

Family $\uparrow$ Mimetaspididae Stensiö 1958

$†$ Mimetaspididae Stensiö 1958: 213 (family) †Mimetaspis Stensiö 1958

Family $\uparrow$ Pattenaspididae Stensiö 1958

$\nmid$ Pattenaspididae Stensiö 1958: 216 (family) †Pattenaspis Stensiö 1958 [changed to $\dagger$ Pattenaspidae by Fowler 1965a: 177]

Family $\uparrow$ Benneviaspididae Denison 1951, spelling in prevailing recent practice

$\uparrow$ Benneviaspinae Denison 1951: 190 (subfamily) †Benneviaspis Stensiö 1927 [family name also seen as $\dagger$ Benneviaspididae]

Family $\uparrow$ Boreaspididae Berg 1955, spelling in prevailing recent practice

$\uparrow$ Boreaspidae Berg 1955: 17 (family) †Boreaspis Stensiö 1927 [corrected to $†$ Boreaspididae by Stensiö 1958: 213]

$\dagger$ Hoelaspididae Stensiö 1958: 214 (family) †Hoelaspis Stensiö 1927

\section{CLASS †GALEASPIDOMORPHI}

Incertae sedis:

$\dagger$ Hyperaspididae Pan 1992: 64 (family) †Hyperaspis Pan 1992 [type genus preoccupied by Hyperaspis

Redtenbacher 1843 in Coleoptera Coccinellidae; invalid, Art. 39]

\section{Order $\dagger$ Hanyangaspidiformes}

Family $\uparrow$ Hanyangaspididae Pan \& Liu 1975, spelling in prevailing recent practice

$\dagger$ Hanyangaspidae Pan \& Liu in Pan, Wang \& Liu 1975: 147 (family) $†$ Hanyangaspis Pan \& Liu 1975 [family name also seen as $\uparrow$ Hanyangaspididae]

Family $\uparrow$ Xiushuiaspididae Pan \& Wang 1983, spelling in prevailing recent practice

$\dagger$ Xiushuiaspidae Pan \& Wang 1983: 505 (family) †Xiushuiaspis Pan \& Wang 1983 [family name also seen as $\dagger$ Xiushuiaspididae]

Family $\uparrow$ Dayongaspididae Pan \& Zeng 1985, spelling in prevailing recent practice

$\dagger$ Dayongaspidae Pan \& Zeng 1985: 207, 212 (family) †Dayongaspis Pan \& Zeng 1985 [family name also seen as $\uparrow$ Dayongaspididae]

\section{Order $\uparrow$ Eugaleaspidiformes}

Family $\uparrow$ Sinogaleaspididae Pan \& Wang 1980, spelling in prevailing recent practice

$\uparrow$ Sinogaleaspidae Pan \& Wang 1980: 10 (family) †Sinogaleaspis Pan \& Wang 1980 [family name also seen as $\uparrow$ Sinogaleaspididae]

Family $\uparrow$ Eugaleaspididae Liu 1980, spelling in prevailing recent practice

$\uparrow$ Galeaspidae Liu 1965: 125, 130 (family) †Galeaspis Liu 1965 [type genus preoccupied by $\dagger$ Galeaspis Ivshin 1955 in Trilobita; invalid, Art. 39]

$\dagger$ Liuaspidinidae [†Liuaspididae] Whitley 1976: 48 (family) $†$ Liuaspis Whitley 1976 [type genus preoccupied by Liuaspis Borchsenius 1960 in Hemiptera; invalid, Art. 39]

$†$ Eugaleaspidae Liu 1980: 256 (family) †Eugaleaspis Liu 1980 [family name also seen as $\dagger$ Eugaleaspididae]

$\uparrow$ Tridensaspidae Liu 1986: 2, 9 (family) †Tridensaspis Liu 1986 [family name sometimes seen as $\dagger$ Tridensaspididae] 


\section{Superorder $\uparrow$ Polybranchiaspidida}

Incertae sedis:

Family $\uparrow$ Geraspididae Pan \& Chen 1993

$\dagger$ Geraspididae Pan \& Chen 1993: 226, 230 (family) †Geraspis Pan \& Chen 1993

\section{Order $\uparrow$ Polybranchiaspidiformes}

Family $\uparrow$ Pentathyraspididae Pan 1992, spelling in prevailing recent practice

$\uparrow$ Pentathyraspididae Pan 1992: 58 (family) †Pentathyraspis Pan 1992 [family name also seen as $\uparrow$ Pentathyraspidae]

$\uparrow$ Microhoplonaspididae Pan 1992: 60 (family) †Microhoplonaspis Pan 1992

Family $\uparrow$ Duyunolepididae Pan \& Wang 1982, spelling in prevailing recent practice

$\uparrow$ Duyunoaspidae Pan \& Wang 1978: 301 (family) †Duyunaspis Pan \& Wang 1978 [type genus preoccupied by $\uparrow$ Duyunaspis Zhang \& Qian 1977 in Trilobita; invalid, Art. 39]

$\uparrow$ Duyunolepidae Pan \& Wang 1982: 370 (family) $†$ Duyunolepis Pan \& Wang 1982 [family name also seen as $\uparrow$ Duyunolepididae]

Family $\uparrow$ Polybranchiaspididae Liu 1965 , spelling in prevailing recent practice

$\dagger$ Polybranchiaspidae Liu 1965: 128, 133 (family) †Polybranchiaspis Liu 1965 [family name also seen as $\uparrow$ Polybranchiaspididae]

$\uparrow$ Cyclodiscaspidae Liu 1975: 211 (family) †Cyclodiscaspis Liu 1975 [family name sometimes seen as $†$ Cyclodiscaspididae]

\section{Order $\uparrow$ Zhaotongaspidiformes}

Family $\uparrow$ Zhaotongaspididae Wang \& Zhu 1994

$\nmid$ Zhaotongaspididae Wang \& Zhu 1994: 234 (family) †Zhaotongaspis Wang \& Zhu 1994

\section{Order $\uparrow$ Huananaspidiformes}

Family $†$ Sanchaspididae Pan \& Wang 1981, spelling in prevailing recent practice

$\dagger$ Sanchaspidae Pan \& Wang 1981: 114, 120 (family) †Sanchaspis Pan \& Wang 1981 [family name also seen as $\dagger$ Sanchaspididae]

Family $\uparrow$ Gantarostrataspididae Wang \& Wang 1992, spelling in prevailing recent practice

$\dagger$ Gantarostrataspidae Wang \& Wang 1992: 186, 192 (family) †Gantarostrataspis Wang \& Wang 1992 [family name also seen as $\dagger$ Gantarostrataspididae]

Family $\uparrow$ Sanqiaspididae Liu 1975, spelling in prevailing recent practice

$\dagger$ Sanqiaspidae Liu 1975: 211 (family) †Sanqiaspis Liu 1975 [family name also seen as $†$ Sanqiaspididae]

Family $\uparrow$ Huananaspididae Liu 1973, spelling in prevailing recent practice

$†$ Huananaspidae Liu 1973: 133 (family) †Huananaspis Liu 1973 [family name also seen as $\dagger$ Huananaspididae]

$\dagger$ Lungmenshanaspididae Pan 1992: 45 (family) †Lungmenshanaspis Pan \& Wang 1975

$\uparrow$ Macrothyraspididae Pan 1992: 49 (family) †Macrothyraspis Pan 1992

†Qingmenaspididae Halstead 1993: 580 (family) †Qingmenaspis Pan \& Wang 1981 [name only, published after 1960, not available, Art. 13.1.1]

Family $\uparrow$ Nanpanaspididae Liu 1975, spelling in prevailing recent practice

$\uparrow$ Nanpanaspidae Liu 1975: 203 (family) †Nanpanaspis Liu 1965 [family name also seen as $\dagger$ Nanpanaspididae]

\section{Order $\uparrow$ Pituriaspidiformes}

Family $\uparrow$ Pituriaspididae

$†$ Pituriaspida Young 1991: 86 (Class) [no family-group name] 
$\dagger$ Pituriaspididae Davis 1994: 8 (family) †Pituriaspis Young 1991 [name only, published after 1960, not available, Art. 13.1.1]

$\uparrow$ Neeyambaspididae Davis 1994: 8 (family) $\uparrow$ Neeyambaspis Young 1991 [name only, published after 1960, not available, Art. 13.1.1]

\section{SUPERCLASS GNATHOSTOMATA}

\section{CLASS †PLACODERMI}

Incertae sedis:

$\dagger$ Cratoselachiidae Woodward 1932: 60 (family) $†$ Cratoselache Woodward 1924 [family name sometimes seen as $\uparrow$ Cratoselachidae]

\section{Order $\uparrow$ Brindabellaspida}

Family $\uparrow$ Brindabellaspidae

$\dagger$ Brindabellaspidae Gardiner 1993a: 583 (family) †Brindabellaspis Young 1980 [name only, published after 1960, not available, Art. 13.1.1]

$†$ Brindabellaspididae Davis 1994: 10 (family) †Brindabellaspis Young 1980 [name only, published after 1960, not available, Art. 13.1.1]

\section{Order $\uparrow$ Stensioelliformes}

Family $\uparrow$ Stensioellidae Berg 1940

$\uparrow$ Stensiöellidae Berg 1940: 370 (family) $†$ Stensioella Broili 1933 [type genus as $†$ Stensiöella, name must be corrected Art. 32.5.3; family name sometimes seen as $\dagger$ Stensiollidae]

\section{Order $\uparrow$ Pseudopetalichthyiformes}

Family $\uparrow$ Paraplesiobatidae Berg 1940, spelling in prevailing recent practice

$\uparrow$ Paraplesiobatidae Berg 1940: 363 (family) $†$ Paraplesiobatis Broili 1933 [family name also seen as $\uparrow$ Paraplesiobatididae; correct stem would be Paraplesiobatid-, no correction needed Art. 29.3.1.1]

$\uparrow$ Pseudopetalichthyidae Gregory 1973: 702,718 (family) $†$ Pseudopetalichthys Moy-Thomas 1939 [downgraded from ordinal series?; name only, published after 1960, not available, Art. 13.1.1]

$\uparrow$ Nessariostomidae Gregory 1973: 718 (family) †Nessariostoma Broili 1933 [name only, published after 1960, not available, Art. 13.1.1]

$\dagger$ Pseudopetalichthyidae Goujet 1984a: 213 (family) $†$ Pseudopetalichthys Moy-Thomas 1939 [name only, published after 1960, not available, Art. 13.1.1]

\section{Order $\uparrow$ Acanthothoraci}

Incertae sedis:

†Weejasperaspidae White 1978: 155 (family) †Weejasperaspis White 1978 [family name sometimes seen as $\uparrow$ Weejasperaspididae]

Family $\uparrow$ Radotinidae Arambourg 1958

$\uparrow$ Radotinidae Arambourg 1958: 2003 (family) †Radotina Gross 1950 [also as new family in Obruchev 1964b: 132]

Family $\uparrow$ Kolymaspididae Bystrow 1956, spelling in prevailing recent practice

$\dagger$ Kolymaspidae Bystrow 1956: 7 (family) †Kolymaspis Bystrow 1956 [family name also seen as $†$ Kolymaspididae]

Family $\uparrow$ Palaeacanthaspidae Stensiö 1944, spelling in prevailing recent practice

$\uparrow$ Palaeacanthaspidae Stensiö 1944: 70 (family) †Palaeacanthaspis Brotzen 1934 [family name also seen as $\uparrow$ Palaeacanthaspididae or $\uparrow$ Palaeacanthaspidea]

Family †Hagiangellidae Dupret, Phuong, Thanh, Phong, Janvier \& Clément 2011 
$†$ Hagiangellidae Dupret, Phuong, Thanh, Phong, Janvier \& Clément 2011: 532 (family) $\dagger$ Hagiangella Janvier 2005

\section{Order $\uparrow$ Rhenanida}

Family $\uparrow$ Asterosteidae Woodward 1891

$\uparrow$ Asterosteidae Woodward 1891a: 312 (family) †Asterosteus Newberry 1875

$\uparrow$ Gemuendinidae Traquair 1903: 734 (family) $†$ Gemuendina Traquair 1903

$\dagger$ Jagorinidae Woodward 1932: 59 (family) †Jagorina Jaekel 1921

\section{$\uparrow$ ANTIARCHI}

Incertae sedis:

$†$ Minicranidae ? Jia 2008: 14 (family) †Minicrania Zhu \& Janvier 1996 [correct stem would be Minicrani-; unavailable publication, family-group name not available]

Family $\nmid$ Liujiangolepidae Wang 1987, spelling in prevailing recent practice

$\dagger$ Liujiangolepidae Wang 1987: 82, 88 (family) †Liujiangolepis Wang 1987 [family name also seen as $\nmid$ Liujiangolepididae]

Family $\dagger$ Silurolepidae Zhang, Wang, Wang, Wang \& Zhu 2010, spelling in prevailing recent practice

$\dagger$ Silurolepidae Zhang, Wang, Wang, Wang \& Zhu 2010: 129 (family) †Silurolepis Zhang et al. 2010 [family name also seen as $†$ Silurolepididae]

\section{Order $\uparrow$ Yunnanolepidiformes}

Family $\uparrow$ Yunnanolepididae Gross 1965, spelling in prevailing recent practice

$\uparrow$ Yunnanolepidinae Gross 1965: 77 (subfamily) †Yunnanolepis Liu 1963 [also as a new family in Zhang 1978b: 148; family name also seen as †Yunnanolepidae]

Family $\uparrow$ Chuchinolepididae Zhang 1978, spelling in prevailing recent practice

$\uparrow$ Chuchinolepidae Zhang 1978a: 296 (family) †Chuchinolepis Zhang 1978 [family name also seen as $†$ Chuchinolepididae; author also seen as Chang]

$\uparrow$ Qujinolepidae Zhang 1978b: 173 (family) †Qujinolepis Zhang 1978 [family name sometimes seen as $†$ Qujinolepididae]

$\uparrow$ Procondylolepidae Zhang 1984: 82 (family) †Procondylolepis Zhang 1984

\section{Order Sinolepiformes}

Family $\uparrow$ Sinolepidae Liu \& Pan 1958, spelling in prevailing recent practice

$\uparrow$ Sinolepidae Liu \& Pan 1958: 8 (family) †Sinolepis Liu \& Pan 1958 [family name also seen as $\dagger$ Sinolepididae]

$\dagger$ Grenfellaspididae Davis 1994: 18 (family) †Grenfellaspis Ritchie, Wang, Young \& Zhang 1992 [name only, published after 1960, not available, Art. 13.1.1]

\section{Order $\uparrow$ Asterolepidiformes}

Family $\uparrow$ Asterolepididae Traquair 1888, name and spelling in prevailing recent practice

†Chelonichthyda Asmuss 1856: 10 (family) †Chelonichthys Agassiz 1844 [type genus inferred from the stem, Art. 11.7.1.1]

$\uparrow$ Asterolepidae Traquair 1888a: 485 (family) $\uparrow$ Asterolepis Eichwald 1840 [family name also seen as $\uparrow$ Astrolepidae, $\uparrow$ Astrolepididae and $\uparrow$ Asterolepididae]

Family $\uparrow$ Remigolepididae Stensiö 1931, spelling in prevailing recent practice

$\uparrow$ Remigolepidae Stensiö 1931: 156 (family) †Remigolepis Stensiö 1931 [family name also seen as $†$ Remigolepididae]

Family $\dagger$ Lepadolepididae Berg 1955, spelling in prevailing recent practice

$\dagger$ Ceratolepidae Gross 1933: 16 (family) †Ceratolepis Gross 1933 [type genus preoccupied by Ceratolepis Chapuis 1873 in Coleoptera; invalid, Art. 39] 
$\dagger$ Lepadolepinae Berg 1955: 38 (subfamily) †Lepadolepis White \& Moy-Thomas 1940 [senior objective synonym of $\uparrow$ Ceratolepichthyinae Fowler 1965 ; family name also seen as $\dagger$ Lepadolepididae]

†Ceratolepichthyinae Fowler 1965b: 347 (subfamily) †Ceratolepichthys Whitley 1940 [junior objective synonym of $\uparrow$ Lepadolepinae Berg 1955, invalid, Art. 61.3.2]

Family $\uparrow$ Pterichthyodidae Stensiö 1948

$\uparrow$ Pterichthidae M’Coy in Sedgwick \& M'Coy 1855: 579 (family) †Pterichthys Agassiz 1841 [type genus preoccupied by Pterichthys Swainson 1839 in fishes Apistidae; invalid, Art. 39; family name sometimes seen as $\uparrow$ Pterichthyidae]

$\uparrow$ Ceraspidae Woodward 1891a: 233 (family) †Ceraspis Schlüter 1887 [type genus preoccupied by Ceraspis Le Peletier \& Audinet Serville 1825 in Coleoptera; invalid, Art. 39; family name sometimes seen as $\dagger$ Ceraspididae]

$\uparrow$ Byssacanthidae Gross 1937: 19 (family) $\uparrow$ Byssacanthus Agassiz 1845 [type genus inferred from the stem, Art. 11.7.1.1; name only, but used as valid by Romer 1945: 575 and (as subfamily) by Stensiö 1948: 219 and by Gross 1965: 15 Art. 13.2.1; subfamily name sometimes seen as $\dagger$ Byssacanthidinae]

$\uparrow$ Cornaspidae Whitley 1940: 242 (family) †Cornaspis Whitley 1940 [name only, but as substitute for $\uparrow$ Ceraspidae; also used as valid by Fowler 1965b: 346 Art. 13.2.1; senior objective synonym of $\uparrow$ Grossaspidae Stensiö 1959]

$\dagger$ Pterichthyodidae Stensiö 1948: 38 (family) †Pterichthyodes Bleeker 1859

$\dagger$ Gerdalepinae Stensiö 1948: 219 (subfamily) †Gerdalepis Hoffmann 1916 [family name sometimes seen as $\dagger$ Gerdalepididae]

$\dagger$ Grossaspidae Stensiö 1959: 7 (family) †Grossaspis White \& Moy-Thomas 1940 [name only; junior objective synonym of $\dagger$ Cornaspidae Whitley 1940, invalid, Art. 61.3.2]

Family $\uparrow$ Asperaspididae Moloshnikov 2012

$\nmid$ Asperaspididae Moloshnikov 2012: 1124 (family) †Asperaspis Panteleyev 1993

Family $\uparrow$ Pambulaspidae Young 2010

$\uparrow$ Pambulaspididae Davis 1994: 52 (family) †Pambulaspis Young 1983 [name only, published after 1960, not available, Art. 13.1.1]

$†$ Pambulaspidae Young 2010: 85 (family) †Pambulaspis Young 1983

\section{Order $\dagger$ Bothriolepidiformes}

Family $\uparrow$ Dianolepididae Long 1983, name in prevailing recent practice

$\uparrow$ Wudinolepidae Chang 1965: 1, 6 (family) †Wudinolepis Chang 1965 [family name sometimes seen as $\uparrow$ Wudinolepididae]

$\uparrow$ Dianolepididae Long 1983a: 318 (family) $\uparrow$ Dianolepis Chang 1965

$\dagger$ Jiangxilepidae Zhang \& Liu 1991: 196 (family) †Jiangxilepis Zhang \& Liu 1991 [family name sometimes seen as $\dagger$ Jiangxilepididae]

$\dagger$ Tenizolepidinae Moloshnikov 2012: 1131 (subfamily) †Tenizolepis Malinovskaja 1977

Family $\uparrow$ Microbrachiidae Stensiö 1931

$\uparrow$ Microbrachiinae Stensiö 1931: 157 (subfamily) †Microbrachius Traquair 1888

Family $\uparrow$ Bothriolepididae Cope 1886

$\uparrow$ Bothriolepididae Cope 1886: 1031 (family) †Bothriolepis Eichwald 1840 [family name sometimes seen as $\uparrow$ Bothriolepidae]

$†$ Monarolepidinae Moloshnikov 2012: 1180 (subfamily) †Monarolepis Young 1988

$\nmid$ Livnolepidinae Moloshnikov 2012: 1180 (subfamily) †Livnolepis Moloshnikov 2008

Family $\uparrow$ Tubalepididae Moloshnikov 2011

$\dagger$ Tubalepididae Moloshnikov 2011: 303 (family) †Tubalepis Panteleyev 2003 


\section{Order $\uparrow$ Macropetalichthyiformes}

Family $\uparrow$ Macropetalichthyidae Eastman 1898

$\uparrow$ Acanthaspidae Woodward 1891: 3 (family) †Acanthaspis Newberry 1875 [type genus preoccupied by Acanthaspis Amyot \& Audinet Serville 1843 in Hemiptera; invalid, Art. 39]

$\uparrow$ Macropetalichthidae Eastman 1898: 372 (family) †Macropetalichthys Norwood \& Owen 1846 [correct stem is Macropetalichthy-]

†Polyaspidae Heintz 1929: 25, 69 (family) ? †Polyaspis ?? [no stem of the type genus, not available, Art. 11.7.1.1; also Polyaspis preoccupied by Berlese 1882 in mites Acari, then invalid, Art. 39]

$†$ Lunaspidae Gross 1933: 28 (family) †Lunaspis Broili 1929 [name only, but used as valid by Fowler 1965b: 356 Art. 13.2.1]

$\dagger$ Heintzaspidae Strand 1942: 384 (family) $\uparrow$ Heintzaspis Strand 1932

Family $\uparrow$ Quasipetalichthyidae Liu 1991

†Quasipetalichthyidae Liu 1991: 167 (family) $†$ Quasipetalichthys Liu 1973

\section{Order $\uparrow$ Ptyctodontiformes}

Family $\uparrow$ Ptyctodontidae Woodward 1891

$\uparrow$ Ptyctodontidae Woodward 1891a: 37 (family) $\uparrow$ Ptyctodus Pander 1858 [family name sometimes seen as $†$ Pyctodontidae]

$\dagger$ Rhynchodonta Jaekel 1907: 186 (Gruppe?) ? [not $\uparrow$ Rhynchodus Newberry 1873] [no stem of the type genus, not available, Art. 11.7.1.1]

$\uparrow$ Aulacosteidae Fowler 1947: 6 (family) $\uparrow$ Aulacosteus Eichwald 1846

\section{$\uparrow$ ARTHRODIRA}

Incertae sedis:

$\uparrow$ Mediaspidae Heintz 1929: 25, 66 (family) †Mediaspis Heintz 1929 [family name sometimes seen as $\dagger$ Mediaspididae]

$\dagger$ Pholidosteiden Jaekel 1907: 176 (family?) †Pholidosteus Jaekel 1907 [published not in latinized form after 1899, not available]

$\uparrow$ Pholidosteidae Gross 1932: 11 (family) †Pholidosteus Jaekel 1907

$\uparrow$ Prescottaspidae White 1961: 297 (family) †Prescottaspis White 1961 [corrected by Miles 1969: 125 to $\uparrow$ Prescottaspididae]

\section{Order $\uparrow$ Actinolepidiformes}

Family $\uparrow$ Antarctaspididae White 1968, spelling in prevailing recent practice

$\uparrow$ Antarctaspidae White 1968: 18 (family) †Antarctaspis White 1968 [family name also seen as $\uparrow$ Antarctaspididae]

Family $\uparrow$ Wuttagoonaspididae Ritchie 1973, spelling in prevailing recent practice

†Wuttagoonaspidae Ritchie 1973: 70 (family) †Wuttagoonaspis Ritchie 1973 [family name also seen as $\uparrow$ Wuttagoonaspididae]

Family $†$ Kujdanowiaspididae Berg 1955, spelling in prevailing recent practice

$\dagger$ Kujdanowiaspidae Berg 1955: 41 (family) $†$ Kujdanowiaspis Stensiö 1942 [family name also seen as $†$ Kujdanowiaspididae]

Family $\uparrow$ Gavinaspididae Dupret \& Zhu 2008

†Gavinaspididae Dupret \& Zhu 2008: 259 (family) †Gavinaspis Dupret \& Zhu 2008

Family $\uparrow$ Phyllolepididae Woodward 1891, spelling in prevailing recent practice

$†$ Phyllolepidae Woodward 1891a: 313 (family) †Phyllolepis Agassiz 1844 [family name also seen as $†$ Phyllolepididae] 
Family $\uparrow$ Actinolepididae Gross 1940, spelling in prevailing recent practice

$\uparrow$ Actinolepidae Gross 1940: 54 (family) †Actinolepis Agassiz 1845 [family name also seen as $\dagger$ Actinolepididae]

\section{Order $\uparrow$ Phlyctaeniiformes}

Suborder $\uparrow$ Phlyctaenioidei

Family $\uparrow$ Phlyctaeniidae Fowler 1947 (1929), name in prevailing recent practice, Art. 40.2

$\dagger$ Phlyctaenaspiden Jaekel 1903: 58 (? family) †Phlyctaenaspis Traquair 1890 [published not in latinized form after 1899, not available]

$\dagger$ Phlyctaenaspiden Jaekel 1907: 176 (? family) $†$ Phlyctaenaspis Traquair 1890 [published not in latinized form after 1899, not available]

$\dagger$ Phlyctaenaspi Jaekel 1911: 46 (suborder) [no family-group name]

$\uparrow$ Monaspidae Heintz 1929: 25, 26 (family) $\uparrow$ Monaspis Heintz 1929 [type genus preoccupied by Monaspis Smith 1849 in Reptilia; invalid, Art. 39]

$\uparrow$ Phlyctaenaspinae Hay 1929: 651 (subfamily) †Phlyctaenaspis Traquair 1890 [name only, but in reference to †phlyctaenaspids Stensiö 1925, and used as valid by Romer 1945: 574 and by Romer 1966: 348 Art. 13.2.1; senior objective synonym of $\uparrow$ Phlyctaeniidae]

$\dagger$ Jaekelaspidae Heintz 1932: 210 (family) †Jaekelaspis Heintz 1929

$\dagger$ Heterogaspidae Strand 1933: 120 (family) †Heterogaspis Strand 1932

$\dagger$ Phlyctaeniidae Fowler 1947: 5 (family) $\uparrow$ Phlyctaenius Traquair 1890 [junior objective synonym of $\uparrow$ Phlyctaenaspinae, invalid, Art. 61.3.2, but name in prevailing recent practice Art. 40.2; $\uparrow$ Phlyctaenius Traquair not preoccupied by $\uparrow$ Phlyctaenium Zittel 1879]

$\dagger$ Williamsaspidae White 1952: 254 (family) †Williamsaspis White 1952 [family name sometimes seen as $\dagger$ Williamsaspididae]

$\uparrow$ Aggeraspididae Miles 1969: 125, 145 (family) $\uparrow$ Aggeraspis Gross 1962 [name only, published after 1960, not available, Art. 13.1.1]

Family $\uparrow$ Arctolepididae Heintz 1937, spelling in prevailing recent practice

$\dagger$ Arctolepidae Heintz 1937: 14 (family) †Arctolepis Eastman 1908 [family name also seen as $\uparrow$ Arctolepididae]

Family $\uparrow$ Gronlandaspididae Obruchev 1964

$†$ Groenlandaspididae Obruchev 1964b: 143 (family) †Gronlandaspis Heintz 1932 [type genus as $\dagger$ Groenlandaspis, name must be corrected Art. 32.5.3 and 32.5.2.1; type genus corrected to $\uparrow$ Gronlandaspis by Snitting \& Blom 2009: 269]

$\uparrow$ Tiaraspididae Miles 1969: 125, 145 (family) †Tiaraspis Gross 1962 [name only, published after 1960, not available, Art. 13.1.1; family name sometimes seen as †Tiaraspidae]

Family $\uparrow$ Arctaspididae Goujet 1984

$†$ Arctaspididae Goujet 1978 (family) †Arctaspis Heintz 1929 [unpublished PhD thesis, not available]

$\uparrow$ Arctaspididae Goujet 1984b: 251 (family) †Arctaspis Heintz 1929

Family $\dagger$ Holonematidae Obruchev 1933, spelling in prevailing recent practice

$\uparrow$ Holonemidae Obruchev 1933: 97 (family) $\uparrow$ Holonema Newberry 1890 [family name also seen as $\dagger$ Holonematidae]

\section{Suborder $\uparrow$ Brachythoraci}

Family $\uparrow$ Homostiidae Eastman 1907

$\dagger$ Homosteiden Jaekel 1903: 58 (? family) $†$ Homostius Asmuss 1856 [type genus as $\uparrow$ Homosteus; published not in latinized form after 1899, not available]

$\uparrow$ Homosteidae Eastman 1907: 23 (family) $\uparrow$ Homostius Asmuss 1856 [type genus as $\uparrow$ Homosteus, name must be corrected Art. 32.5.3; corrected to $\uparrow$ Homostiidae by Heintz 1934: 85, confirmed by Camp \& Vanderhoof 1940: 489]

$\dagger$ Euleptaspididae Obruchev 1964b: 144 (family) †Euleptaspis White \& Moy-Thomas 1940 
$\uparrow$ Burrinjucosteidae White 1978: 177 (family) †Burrinjucosteus White 1978

$\uparrow$ Goodradigbeeonidae White 1978: 162 (family) †Goodradigbeeon White 1978

Family $\uparrow$ Buchanosteidae White 1952

$\uparrow$ Buchanosteidae White 1952: 266 (family) †Buchanosteus Stensiö 1945

$\uparrow$ Taemasosteidae White 1952: 276 (family) †Taemasosteus White 1952

Family $\uparrow$ Parabuchanosteidae Long, Mark-Kurik \& Young 2014

$\uparrow$ Parabuchanosteidae Long, Mark-Kurik \& Young 2014: 37 (family) †Parabuchanosteus

White \& Toombs 1972

Family $\dagger$ Gemuendenaspidae Miles 1962

†Gemuendenaspidae Miles 1962: 74 (family) †Gemuendenaspis Miles 1962 [family name sometimes seen as $†$ Gemuendenaspididae]

$\dagger$ Coccosteomorphi

Family $\uparrow$ Coccosteidae Asmuss 1856

$\uparrow$ Coccosteida Asmuss 1856: 6 (family) $†$ Coccosteus Agassiz 1841 [type genus inferred from the stem, Art. 11.7.1.1]

? †Solenosteidae Heintz 1932: 210 (family) ? †"Solenosteus" [no valid type genus, not available, Art. 11.7.1.1] [family uncertain]

$\uparrow$ Millerosteidae Stensiö 1963: 392 (family) †Millerosteus Stensiö 1959

Family $\uparrow$ Torosteidae Gardiner \& Miles 1990

$\uparrow$ Torosteidae Gardiner \& Miles 1990: 159 (family) †Torosteus Gardiner \& Miles 1990

Family $\uparrow$ Trematosteidae Gross 1932

$\dagger$ Trematosteidae Gross 1932: 30 (family) †Trematosteus Gross 1932

Family $\uparrow$ Camuropiscidae Dennis \& Miles 1979

$\uparrow$ Camuropiscidae Dennis \& Miles 1979: 297 (family) †Camuropiscis Dennis \& Miles 1979

$\dagger$ Incisoscutidae Denison 1984: 396 (family) †Incisoscutum Dennis \& Miles 1981 [also as a new family in Gardiner 1993a: 587]

Family $\uparrow$ Mylostomatidae Woodward 1891

$\uparrow$ Mylostomatidae Woodward 1891a: 315 (family) $†$ Mylostoma Newberry 1883 [family name sometimes seen as $\uparrow$ Mylostomidae]

$\uparrow$ Trachosteidae Dean 1901: 120 (family) †Trachosteus Newberry 1890

$\uparrow$ Kendrickichthyidae Gardiner 1993a: 587 (family) †Kendrickichthys Dennis \& Miles 1980 [name only, published after 1960, not available, Art. 13.1.1]

Family $\uparrow$ Bungartiidae Denison 1975

$\dagger$ Bungartiidae Denison 1975: 19 (family) †Bungartius Dunkle 1947

$\dagger$ Pachyosteomorphi

Family $\uparrow$ Rhachiosteidae Stensiö 1963

$\dagger$ Rhachiosteidae Stensiö 1963: 392 (family) $\uparrow$ Rhachiosteus Gross 1938 [family name sometimes seen as $\dagger$ Rachiosteidae]

Family $\uparrow$ Panxiosteidae Wang 1979

$\dagger$ Panxiosteidae Wang 1979: 179 (family) †Panxiosteus Wang 1979

†Plourdosteidae Vézina 1990: 677 (family) †Plourdosteus Ørvig 1951

Family $\dagger$ Dunkleosteidae Stensiö 1963, name in prevailing recent practice

$\dagger$ Heterosteiden Jaekel 1903: 58 (family) $\uparrow$ Heterostius Asmuss 1856 [type genus as $\uparrow$ Heterosteus; published not in latinized form after 1899, not available]

$\dagger$ Heterostii Jaekel 1919: 98 (no family-group name)

$\dagger$ Heterosteidae Gross 1932: 55 (family) $\uparrow$ Heterostius Asmuss 1856 [type genus as $\uparrow$ Heterosteus, name must be corrected Art. 32.5.3; corrected to $\dagger$ Heterostiidae by Gross 1933: 38, confirmed by Berg 1940: 368] 
$\dagger$ Ichthyosauroididae Fowler 1947: 5 (family) †Ichthyosauroides Kutorga 1837

$\dagger$ Dunkleosteidae Stensiö 1963: 387 (family) $†$ Dunkleosteus Lehman 1956

$\dagger$ Hussakofiidae Obruchev 1964b: 148 (family) †Hussakofia Cossman 1910

$\dagger$ Aspinothoracidi

Family $\uparrow$ Dinichthyidae Newberry 1885

†Dinichthidae Newberry 1885: 28 (family) †Dinichthys Newberry 1868 [corrected to $\dagger$ Dinichthyidae by Cope 1889: 856; correct stem is Dinichthy-]

Family $\uparrow$ Brachydeiridae Gross 1932

$\uparrow$ Auchenosteidae Jaekel 1911: 48 (family) $\uparrow$ Auchenosteus Jaekel 1919 [no valid type genus, not available, Art. 11.7.1.1]

$\uparrow$ Brachydiridae Gross 1932: 34 (family) †Brachydeirus Koenen 1880 [type genus as $\dagger$ Brachydirus, name must be corrected Art. 32.5.3; corrected to $\dagger$ Brachydeiridae by Fowler 1947: 6, confirmed by Fowler 1965b: 378]

Family $\uparrow$ Braunosteidae Stensiö 1963

$†$ †raunosteidae Stensiö 1963: 387 (family) †Braunosteus Stensiö 1959 [“represents a separate family", but not named by Stensiö 1959: 113]

Family $†$ Hadrosteidae Gross 1932

$\dagger$ Hadrosteidae Gross 1932: 26 (family) †Hadrosteus Gross 1932 [family name sometimes seen as $\dagger$ Hadrostiidae]

Family $\uparrow$ Selenosteidae Dean 1901

$\uparrow$ Selenosteidae Dean 1901: 120 (family) †Selenosteus Dean 1901

$\uparrow$ Pachyosteidae Gross 1932: 16 (family) †Pachyosteus Jaekel 1903

$\dagger$ Rhinosteidae Stensiö 1963: 387 (family) †Rhinosteus Jaekel 1911

Family †Leiosteidae Stensiö 1963

? †Leiosteidae Jaekel 1919: 106 (family) ? †Leiosteus Gross 1932 [type genus inferred from the stem, Art. 11.7.1.1; no valid type genus, not available, Art. 11.7.1.1] [family uncertain]

$\dagger$ Leiosteidae Stensiö 1963: 387 (family) †Leiosteus Gross 1932

$†$ Erromenosteidae Obruchev 1964b: 150 (family) $†$ Erromenosteus Jaekel 1919

Family $†$ Leptosteidae Gross 1932

$\dagger$ Leptosteidae Jaekel 1911: 48 (family) †Leptosteus Jaekel 1919 [no valid type genus, not available, Art. 11.7.1.1]

$†$ Leptosteidae Gross 1932: 27 (family) †Leptosteus Jaekel 1919

Family $\uparrow$ Titanichthyidae Dean 1901

$\dagger$ Titanichthyidae Dean 1901: 120 (family) †Titanichthys Newberry 1885

$\dagger$ Leiosteiden Jaekel 1919: 90 (family) †Oxyosteus Jaekel 1911 [no stem of type genus, not available, Art. 11.7.1.1; and published not in latinized form after 1899, not available]

†Oxyosteidae Gross 1932: 39 (family) †Oxyosteus Jaekel 1911

$\uparrow$ Synaucheniidae Gross 1932: 44 (family) †Synauchenia Jaekel 1919 [name only, but used as valid by Gross 1933: 45 (as †Synauchenidae), by Berg 1940: 368 and by Fowler 1965b: 379 Art. 13.2.1]

Family $\uparrow$ Bulbocanthidae Young \& Goujet 2003

$\dagger$ Bulbocanthidae Young \& Goujet 2003: 24 (family) $†$ Bulbocanthus Bryant 1932

\section{CLASS $\uparrow$ ACANTHODII}

Incertae sedis:

†Onchidae Jordan 1923: 92 (family) †Onchus Agassiz 1837

Family $\uparrow$ Machaeracanthidae Burrow \& Young 2005

$\uparrow$ Machaeracanthidae Burrow \& Young 2005: 17 (family) $\uparrow$ Machaeracanthus Newberry 1857 
VAN DER LAAN R., Family-group names of fossil fishes

Family $\uparrow$ Sinacanthidae Zhu 1998

$\uparrow$ Sinacanthidae Zhu 1998: 161 (family) †Sinacanthus Pan 1959

Family $\uparrow$ Tchunacanthidae Karatajūtè-Talimaa \& Smith 2003

$\dagger$ Lenacanthidae Karatajūtè-Talimaa \& Smith 2003: 281 (family) †Lenacanthus Karatajūtè-

Talimaa \& Smith 2003

$\uparrow$ Tchunacanthidae Karatajūtè-Talimaa \& Smith 2003: 286 (family) $†$ Tchunacanthus Karatajūtè-

Talimaa \& Smith 2003 [Valiukevičius \& Burrow 2005: 642 gave precedence to Tchunacanthidae over Lenacanthidae]

\section{Order $\uparrow$ Diplacanthiformes}

Family †Culmacanthidae Long 1983

$\uparrow$ Culmacanthidae Long 1983b: 52 (family) †Culmacanthus Long 1983

Family $\dagger$ Diplacanthidae Bleeker 1859

†Diplacanthidae Bleeker 1859: XVII (family) †Diplacanthus Agassiz 1844 [family name sometimes seen as $\dagger$ Diplocanthidae]

Family $\dagger$ Gladiobranchidae Bernacsek \& Dineley 1977

$\uparrow$ Gladobranchidae [ $\uparrow$ Gladiobranchidae] Bernacsek \& Dineley 1977: 13 (family) $\dagger$ Gladiobranchus Bernacsek \& Dineley 1977

\section{Order $\dagger$ Ischnacanthiformes}

Family $\uparrow$ Tetanopsyridae Gagnier, Hanke \& Wilson 1999

$\uparrow$ Tetanopsyridae Gagnier, Hanke \& Wilson 1999: 83 (family) †Tetanopsyrus Gagnier, Hanke \& Wilson 1999

Family †Ischnacanthidae Woodward 1891

$\dagger$ Ischnacanthidae Woodward 1891a: 20 (family) †Ischnacanthus Powrie 1864

$\dagger$ Ictinocephalidae Jordan 1923: 91 (family) †Ictinocephalus Page 1864 [name in synonymy; treated as available before 1961?; not available, Art. 11.6.1]

Family $\uparrow$ Acritolepidae Valiukevičius \& Burrow 2005

$\uparrow$ Acritolepidae Valiukevičius \& Burrow 2005: 644 (family) $†$ Acritolepis Valiukevičius 2003

[family name sometimes seen as $\uparrow$ Acritolepididae]

Family †Poracanthodidae Vergoossen 1997

†Poracanthodidae Vergoossen 1997: 44 (family) †Poracanthodes Brotzen 1934

\section{Order $\uparrow$ Acanthodiformes}

Family $\uparrow$ Mesacanthidae Moy-Thomas 1939

$\uparrow$ Mesacanthidae Moy-Thomas 1939: 34 (family) †Mesacanthus Traquair 1888

Family $\uparrow$ Cheiracanthidae Bleeker 1859

$\uparrow$ Cheiracanthoidei Bleeker 1859: XVII (family) $†$ Cheiracanthus Agassiz 1835

Family $\uparrow$ Acanthodidae Agassiz 1844

$\uparrow$ Acanthodiens Agassiz 1844 Tome II pt. 1: 308 (family) †Acanthodes Agassiz 1833 [latinized to $\uparrow$ Acanthoidini by Bonaparte 1846: 4 (subfamily), latinized to $\uparrow$ Acanthodei by Owen 1846: 50, latinized to $\uparrow$ Acanthodides by Giebel 1846: 65, latinized to $\uparrow$ Acanthodii by Giebel 1847: 227, latinized to $\uparrow$ Achantodontini [ $\uparrow$ Acanthodontini] by Bonaparte 1850a: 455; considered valid with this authorship by Müller 1846: 122, 151, by Bronn 1849: 656 and by Pander 1860: 69 Art. 11.7.2; senior objective synonym of †Acanthoessidae Hay 1902]

$\uparrow$ Acanthoëssidae Hay 1902: 273 (family) †Acanthoessus Agassiz 1832 [type genus as $\uparrow$ Acanthoëssus, name must be corrected Art. 32.5.3 to †Acanthoessidae; junior objective synonym of $\uparrow$ Acanthodidae Agassiz 1844, invalid, Art. 61.3.2]

$\dagger$ Howittacanthidae Zajíc 1995: 168 (family) †Howittacanthus Long 1986 


\section{Order $\uparrow$ Climatiiformes}

Family $\uparrow$ Climatiidae Berg 1940, name in prevailing recent practice

$\uparrow$ Brachyacanthidae Moy-Thomas 1939: 34 (family) †Brachyacanthus Egerton 1860

$\uparrow$ Climatiidae Berg 1940: 373 (family) $\uparrow$ Climatius Agassiz 1845

$\uparrow$ Parexidae Berg 1940: 373 (family) †Parexus Agassiz 1845

Family $\uparrow$ Gyracanthidae Woodward 1906

$\dagger$ Gyracanthidae Woodward 1906: 3 (family) †Gyracanthus Agassiz 1835 [family name sometimes seen as $\dagger$ Gyracanthididae]

$\dagger$ Gyracanthididae Miles 1971: 28 (family) †Gyracanthides McCoy 1890

Family $\uparrow$ Vesperaliidae Valiukevičius \& Burrow 2005

†Vesperaliidae Valiukevičius \& Burrow 2005: 643 (family) †Vesperalia Valiukevičius 2004

Family $†$ Euthacanthidae Berg 1940

$\uparrow$ Euthacanthidae Berg 1940: 373 (family) †Euthacanthus Powrie 1864

Family $\dagger$ Brochoadmonidae Bernacsek \& Dineley 1977

$\uparrow$ Brochoadmonidae Bernacsek \& Dineley 1977: 17 (family) †Brochoadmones Bernacsek \&

Dineley 1977

\section{CLASS CHONDRICHTHYES}

Incertae sedis:

Family $\uparrow$ Bandringidae Zangerl 1969

$\uparrow$ Bandringidae Zangerl 1969: 157 (family) †Bandringa Zangerl 1969

Family $†$ Kathemacanthidae Gagnier \& Wilson 1996

$\dagger$ Kathemacanthidae Gagnier \& Wilson 1996: 243 (family) †Kathemacanthus Gagnier \& Wilson 1996

Family $\uparrow$ Palaeospondylidae Gill 1896

$†$ Palaeospondylidae Gill 1896a: 11 (family) †Palaeospondylus Traquair 1890

Family $\uparrow$ Polysentoridae Zangerl 1979

$\uparrow$ Polysentoridae Zangerl 1979: 458, 460 (family) †Polysentor Zangerl 1979

Family $\uparrow$ Protodontidae Woodward 1932

$\uparrow$ Protodontidae Woodward 1932: 56 (family) †Protodus Woodward 1892

Family $\uparrow$ Wellingtonellidae Märss, Wilson \& Thorsteinsson 2002

$\dagger$ Wellingtonellidae Märss, Wilson \& Thorsteinsson 2002: 117 (family) †Wellingtonella Märss,

Wilson \& Thorsteinsson 2002

\section{Order $\uparrow$ Altholepidiformes}

Family $\uparrow$ Altholepididae Andreev, Coates, Shelton, Cooper, Smith \& Sansom 2015

$\uparrow$ Altholepididae Andreev, Coates, Shelton, Cooper, Smith \& Sansom 2015: 693 (family)

$\uparrow$ Altholepis Karatajūtè-Talimaa 1997

Family $†$ Tazakidae Andreev, Coates, Shelton, Cooper, Smith \& Sansom 2015

$\uparrow$ Tazakidae Andreev, Coates, Shelton, Cooper, Smith \& Sansom 2015: 694 (family) †Tazakia Andreev, Coates, Shelton, Cooper, Smith \& Sansom 2015 [correct stem would be Tazaki-]

\section{Order $\uparrow$ Elegestolepidida}

Family $\dagger$ Kannathalepididae Märss \& Gagnier 2001

$\dagger$ Kannathalepididae Märss \& Gagnier 2001: 695 (family) †Kannathalepis Märss \& Gagnier 2001

Family $†$ Elegestolepididae Andreev, Coates, Karatajūtè-Talimaa, Shelton, Cooper \& Sansom 2017

$†$ Elegestolepididae Andreev, Coates, Karatajūtè-Talimaa, Shelton, Cooper \& Sansom 2017: e1245664-4 (family) †Elegestolepis Karatajūtè-Talimaa 1973 


\section{Order $\uparrow$ Mongolepidiformes}

Family $\uparrow$ Mongolepididae Karatajūtė-Talimaa \& Novitskaya 1990

$†$ Mongolepididae Karatajūtè-Talimaa \& Novitskaya in Karatajūtè-Talimaa, Novitskaya, Rozman \& Sodov 1990: 79 (family) †Mongolepis Karatajūtè-Talimaa \& Novitskaya 1990 [family name sometimes seen as $\uparrow$ Mongolepidae]

Family $\uparrow$ Shiqianolepidae Sansom, Aldridge \& Smith 2000

$\dagger$ Shiqianolepidae Sansom, Aldridge \& Smith 2000: 259 (family) †Shiqianolepis Sansom, Aldridge \& Smith 2000 [family name sometimes seen as $\dagger$ Shiqianolepididae]

\section{Order $\uparrow$ Polymerolepidiformes}

Family $\uparrow$ Polymerolepididae Karatajūtè-Talimaa 1968

$†$ Polymerolepididae Karatajūtè-Talimaa 1968: 37 (family) †Polymerolepis Karatajūtè-Talimaa 1968

\section{Order $†$ Omalodontiformes}

Family †Omalodontidae Ginter, Liao \& Valenzuela-Ríos 2008

†Omalodontidae Ginter, Liao \& Valenzuela-Ríos 2008: 167 (family) †Omalodus Ginter \& Ivanov 1992

Family $\uparrow$ Aztecodontidae Hairapetian, Ginter \& Yazdi 2008

$\uparrow$ Aztecodontidae Hairapetian, Ginter \& Yazdi 2008: 174 (family) †Aztecodus Long \& Young 1995

\section{Order $\uparrow$ Antarctilamniformes}

Family $\uparrow$ Antarctilamnidae Ginter, Liao \& Valenzuela-Ríos 2008

$\uparrow$ Antarctilamnidae Davis 1994: 58 (family) $\uparrow$ Antarctilamna Young 1982 [name only, published after 1960, not available, Art. 13.1.1]

$\uparrow$ Antarctilamnidae Ginter, Liao \& Valenzuela-Ríos 2008: 169 (family) †Antarctilamna Young 1982

Family $\uparrow$ Coronodontidae Romer 1945

$\uparrow$ Coronodontidae Romer 1945: 576 (family) †Coronodus Bryant 1935 [name only, but used as valid by Schultz 1948: 224, by Harris 1951: 683, by Fowler 1966b: 331, by Romer 1966: 349 and by Andrews et al. 1967: 667 Art. 13.2.1]

\section{Order $\uparrow$ Phoebodontiformes}

Family $\uparrow$ Phoebodontidae Williams 1985 , name in prevailing recent practice

$\uparrow$ Diademodidae Arambourg 1958: 2021 (family) †Diademodus Harris 1951

$\uparrow$ Phoebodontidae Williams 1979 (family) †Phoebodus St. John \& Worthen 1875 [unavailable publication, family-group name not available]

$\uparrow$ Phoebodontidae Williams in Zangerl 1981: 54 (family) †Phoebodus St. John \& Worthen 1875

[name only, published after 1960, not available, Art. 13.1.1]

$\dagger$ Phoebodontidae Williams 1985: 124 (family) †Phoebodus St. John \& Worthen 1875

$\uparrow$ Thrinacodontidae Grogan \& Lund 2008: 971 (family) †Thrinacodus St. John \& Worthen 1875

Family $\uparrow$ Jalodontidae Ginter, Hairapetian \& Klug 2002

$\dagger$ Jalodontidae Ginter, Hairapetian \& Klug 2002: 188 (family) †Jalodus Ginter 1999

Superorder $\uparrow$ Cladoselachimorpha (†Cladodontomorphi)

\section{Order $\uparrow$ Cladoselachiformes}

Family $\uparrow$ Cladoselachidae Dean 1894

†Cladoselachidae Dean 1894: 111 (family) †Cladoselache Dean 1894 


\section{Order $\uparrow$ Symmoriiformes}

Family $\uparrow$ Symmoriidae Dean 1909

$\uparrow$ Symmoriidae Dean 1909: 242 (family) $†$ Symmorium Cope 1893

$†$ Stethacanthidae Lund 1974: 164 (family) †Stethacanthus Newberry 1890

Family $\uparrow$ Falcatidae Zangerl 1990, name in prevailing recent practice

$\dagger$ Deneidae Fournier \& Pruvost 1928: 16 (family) $\dagger$ Denaea Pruvost 1922 [type genus as $\uparrow$ Denea, name must be corrected Art. 32.5.3; corrected to $\uparrow$ Denaeidae by Berg 1940: 376, confirmed by Fowler 1966a: 121]

$\dagger$ Falcatidae Zangerl 1990: 139 (family) $\uparrow$ Falcatus Lund 1985

\section{Superorder $\uparrow$ Ctenacanthimorpha \\ Order $\uparrow$ Ctenacanthiformes}

Family $\uparrow$ Ctenacanthidae Bonaparte 1850 , name in prevailing recent practice

$\uparrow$ Cladodini Giebel 1846: 62 (family) †Cladodus Agassiz 1843 [stem changed to Cladodont- by Bonaparte 1850a: 454, confirmed by Woodward 1889a: 16 and by Lydekker in Nicholson \& Lydekker 1889: 927; †Cladodontidae used after 1900, e.g., by Hay 1902: 267 and by Jordan 1905: 520]

$\uparrow$ Ctenacanthini Bonaparte 1850a: 454 (subfamily) †Ctenacanthus Agassiz 1837 [type genus inferred from the stem, Art. 11.7.1.1; also as a new family in Dean 1909: 242]

$\uparrow$ Tamiobatidae Hay 1902: 315 (family) †Tamiobatis Eastman 1897 [also Woodward in Zittel 1902: 41]

Family $\uparrow$ Heslerodidae Maisey 2010

$\uparrow$ Heslerodidae Maisey 2010: 14 (family) †Heslerodus Ginter 2002 [family name sometimes seen as $\dagger$ Heslerodontidae]

\section{Order $\uparrow$ Squatinactiformes}

Family $†$ Squatinactidae Lund \& Zangerl 1974

$\dagger$ Squatinactidae Lund \& Zangerl 1974: 44 (family) $†$ Squatinactis Lund \& Zangerl 1974 [also as a new family in Cappetta, Duffin \& Zidek 1993: 598]

Superorder $\uparrow$ Xenacanthimorpha $(†$ Pleuracanthodii)

Order $\uparrow$ Bransonelliformes $\uparrow$ Bransonella Harlton 1933

\section{Order $\uparrow$ Xenacanthiformes}

Family $\uparrow$ Diplodoselachidae Dick 1981

†Orthacanthidae Jordan 1923: 91 (family) †Orthacanthus Agassiz 1843 [name in synonymy; treated as available before 1961?; not available, Art. 11.6.1]

$\uparrow$ Diplodoselachidae Dick in Zangerl 1981: 64 (family) †Diplodoselache Dick 1981 [also Dick 1981: 99]

†Orthacanthidae Heyler \& Poplin 1990: 70 (family) †Orthacanthus Agassiz 1843

$†$ Lebachacanthidae Soler-Gijón 1997: 3 (family) †Lebachacanthus Soler-Gijón 1997

Family $\uparrow$ Xenacanthidae Geinitz 1860

$\dagger$ Xenacanthi Geinitz 1860: 468 (family) †Xenacanthus Beyrich 1848 [corrected to $\dagger$ Xenacanthidae by Hoernes 1884: 430]

$†$ Pleuracanthidae Hasse 1878b: 169 (no family-group name)

$†$ Pleuracanthidae Woodward 1889a: 1 (family) †Pleuracanthus Agassiz 1837 [type genus preoccupied by Pleuracanthus Gray 1832 in Coleoptera; invalid, Art. 39] 
Subclass HOLOCEPHALI (Euchondrocephali / Subterbranchialia)

Incertae sedis:

$\dagger$ Desmiodontidae Hansen 1985: 523 (family) †Desmiodus St. John \& Worthen 1875 [also as a new family in Cappetta, Duffin \& Zidek 1993: 596; $\uparrow$ Desmiodus is a nomen dubium, no longer recognized as a valid genus]

Family †Gregoriidae Lund \& Grogan 2004

$\dagger$ Gregoriidae Lund \& Grogan 2004b: 506 (family) †Gregorius Lund \& Grogan 2004

Family $\uparrow$ Harpacanthidae Lund \& Grogan 2004

$\dagger$ Harpacanthidae Lund \& Grogan 2004a: 172 (family) $†$ Harpacanthus Traquair 1886

Family $\uparrow$ Lagarodontidae Lebedev 2008

$\dagger$ Lagarodontidae Lebedev 2008: 201 (family) †Lagarodus Jaekel 1898

\section{Order $\uparrow$ Iniopterygiformes}

Family †Iniopterygidae Zangerl \& Case 1973

$\uparrow$ Iniopterygidae Zangerl \& Case 1973: 6 (family) †Iniopteryx Zangerl \& Case 1973

Family $\uparrow$ Sibyrhynchidae Zangerl \& Case 1973

$\dagger$ †ibyrhynchidae Zangerl \& Case 1973: 35 (family) †Sibyrhynchus Zangerl \& Case 1973

\section{Order $\dagger$ Orodontiformes}

Family $\uparrow$ Leiodontidae Ginter, Hampe \& Duffin 2010

$\dagger$ Leiodontidae Ginter, Hampe \& Duffin 2010: 116 (family) †Leiodus St. John \& Worthen 1875 [not Leiodidae Fleming 1821 in Coleoptera]

Family $†$ Orodontidae de Koninck 1878

†Orodontidae de Koninck 1878: 29 (family) †Orodus Agassiz 1838

\section{Order $\uparrow$ Eugeneodontiformes}

Suborder $\uparrow$ Eugeneodontoidei

Family $\uparrow$ Caseodontidae Zangerl 1981

$\dagger$ Caseodontoidea Zangerl 1981: 79 (superfamily) $†$ Caseodus Zangerl 1981 [also as new family]

Family $\dagger$ Eugeneodontidae Zangerl 1981

\section{Suborder $\uparrow$ Edestoidei}

Family $\uparrow$ Helicoprionidae Karpinsky 1911

$\uparrow$ Helicoprionidae Karpinsky 1911: 1113 (family) †Helicoprion Karpinsky 1899 [family name sometimes seen as $\dagger$ Helicopriidae]

$\uparrow$ Agassizodontidae Zangerl 1981: 86 (family) $\uparrow$ Agassizodus St. John \& Worthen 1875

Family $\uparrow$ Edestidae Leidy 1857

$\dagger$ Edestina Leidy 1857: 302 (family) †Edestus Leidy 1856

\section{Order $\uparrow$ Petalodontiformes}

Family $\uparrow$ Belantseidae Lund 1989

$\uparrow$ Belantseidae Lund 1989: 352 (family) †Belantsea Lund 1989

Family $\uparrow$ Petalodontidae Newberry \& Worthen 1866

$\uparrow$ Petalodontes Giebel 1847: 344 (? subfamily) $\uparrow$ Petalodus Owen 1845 [published not in latinized form before 1900, not available, Art. 11.7.2]

$\uparrow$ Petalodontidae Newberry \& Worthen 1866: 31 (family) $\uparrow$ Petalodus Owen 1845

$\uparrow$ Polyrhizodontinae Jaekel 1898: 49 (subfamily) †Polyrhizodus M’Coy 1848 [subfamily name sometimes seen as $\uparrow$ Polyrhizontinae]

Family $\uparrow$ Pristodontidae Woodward 1889 
$\dagger$ Pristodontidae Woodward 1889a: 62 (family) †Pristodus Davis 1883

$\dagger$ Peripristidae Eastman 1902: 389 (family) $\dagger$ Peripristis St. John 1870

Family †Janassidae Jaekel 1898

$\dagger$ Janassinae Jaekel 1898: 49 (subfamily) †Janassa Münster 1839

Family $\uparrow$ Petalorhynchidae Lund, Grogan \& Fath 2014

$\dagger$ Petalorhynchidae Lund, Grogan \& Fath 2014: 1026 (family) †Petalorhynchus Morris \& Roberts 1862

Family $\uparrow$ Obruchevodidae Lund, Grogan \& Fath 2014

$\dagger$ Obruchevodidae Lund, Grogan \& Fath 2014: 1026 (family) †Obruchevodus Grogan, Lund \& Fath 2014

\section{Order $\uparrow$ Debeeriiformes}

Family $\uparrow$ Debeeriidae Grogan \& Lund 2000

$\uparrow$ Debeeriidae Grogan \& Lund 2000: 220 (family) †Debeerius Grogan \& Lund 2000

\section{Order $\uparrow$ Helodontiformes}

Family $\dagger$ Helodontidae Jaekel 1898

$\dagger$ Helodontinae Jaekel 1898: 50 (subfamily) †Helodus Agassiz 1838

\section{Superorder Holocephalimorpha}

Order $\uparrow$ Psammodontiformes

Family †Psammodontidae de Koninck 1878

$\uparrow$ Psammodonten Pander 1856: 77 (family) $†$ Psammodus Agassiz 1838 [published not in latinized form before 1900, not available, Art. 11.7.2]

$†$ Psammodontidae de Koninck 1878: 38 (family) †Psammodus Agassiz 1838

\section{Order $\uparrow$ Copodontiformes}

Family $\uparrow$ Copodontidae Davis 1883

†Copodontidae Davis 1883: 57 (family) †Copodus Morris \& Roberts 1862

$†$ Cochliodontomorpha

Order $\uparrow$ Squalorajiformes

Family $\uparrow$ Squalorajidae Hasse 1878

$\dagger$ Squalorajidae Hasse 1878a: 247, 257 (family) $†$ Squaloraja Riley 1833 [family name sometimes seen as $†$ Squaloraiidae]

\section{Order $\uparrow$ Chondrenchelyiformes}

Family $\uparrow$ Chondrenchelyidae Jordan 1923

†Chondrenchelyidae Jordan 1923: 92 (family) †Chondrenchelys Traquair 1888

\section{Order $\uparrow$ Menaspiformes}

Family $\uparrow$ Menaspidae Woodward 1891, spelling in prevailing recent practice

$\dagger$ Trachyacanthidae Jaekel 1890: 130 (family) †Menaspis Ewald 1848 [no stem of the type genus, not available, Art. 11.7.1.1]

$\uparrow$ Menaspidae Woodward 1891b: 424 (family) $\uparrow$ Menaspis Ewald 1848 [family name sometimes seen as $\dagger$ Menaspididae or $\dagger$ Manaspidae]

Family $\uparrow$ Deltoptygiidae Patterson 1965

$\dagger$ Deltoptygiidae Patterson 1965: 149 (family) †Deltoptygius Morris \& Roberts 1862

Family $\uparrow$ Traquairiidae Lund \& Grogan 1997

$\dagger$ Traquairiidae Lund \& Grogan 1997: 477 (family) †Traquairius Lund \& Grogan 1997 [family name sometimes seen as $\uparrow$ Traquairidae] 


\section{Order $\uparrow$ Cochliodontiformes}

Family $\uparrow$ Cochliodontidae Owen 1867

$\uparrow$ Cochliodontidae Owen 1867: 59 (family) †Cochliodus Agassiz 1838

$\uparrow$ Rhadamantidae Woodward 1932: 73 (family) †Radamas Münster 1843 [type genus as $\dagger$ Rhadamas, name must be corrected Art. 32.5.3; corrected to $\dagger$ Radamantidae by Berg 1940: 383, confirmed by Fowler 1966b: 317]

$\dagger$ Deltodontidae Zangerl 1981: 42, 46 (family) †Deltodus Morris \& Roberts 1862 [name only, published after 1960, not available, Art. 13.1.1]

Family $\uparrow$ Psephodontidae Jaekel 1898

$†$ Psephodontinae Jaekel 1898: 50 (subfamily) †Psephodus Morris \& Roberts 1862

Chimaerimorpha

\section{Order Chimaeriformes}

\section{Suborder $\uparrow$ Echinochimaeroidei}

Family $\uparrow$ Echinochimaeridae Lund 1977

$\uparrow$ Echinochimaeridae Lund 1977: 198 (family) $†$ Echinochimaera Lund 1977

\section{Suborder $\uparrow$ Myriacanthoidei}

Family $\uparrow$ Myriacanthidae Woodward 1889

$\uparrow$ Myriacanthidae Woodward 1889b: 279 (family) $\uparrow$ Myriacanthus Agassiz 1837

$\uparrow$ Acanthorhinidae Patterson 1965: 146 (family) $\uparrow$ Acanthorhina Fraas 1910

Family $\uparrow$ Chimaeropsidae Jaekel 1919

$\dagger$ Chimaeropsidae Jaekel 1919: 107 (family) $†$ Chimaeropsis Zittel 1887

\section{Suborder Chimaeroidei}

Family Callorhinchidae Garman 1901

$\dagger$ Edaphodontidae Owen 1846: 51 (family) $†$ Edaphodon Buckland 1838 [also as a new subfamily †Edaphodontinae in Stahl 1999: 138]

Family Rhinochimaeridae Garman 1901

Family Chimaeridae Rafinesque 1815

\section{Subclass EUSELACHII}

Incertae sedis:

Family $\nmid$ Listracanthidae Martill, Del Strother \& Gallien 2014

$\dagger$ Listracanthidae Martill, Del Strother \& Gallien 2014: 7 (family) †Listracanthus Newberry \& Worthen 1870

\section{Order $\uparrow$ Protacrodontiformes}

Family $\uparrow$ Protacrodontidae Zangerl 1981

$\uparrow$ Protacrodontoidea Zangerl 1981: 61 (superfamily) †Protacrodus Jaekel 1925 [also as a new family, †Protacrodontidae, in Cappetta, Duffin \& Zidek 1993: 596]

\section{Infraclass $\dagger$ HYBODONTA}

Order $\uparrow$ Hybodontiformes

Family $\uparrow$ Hybodontidae Agassiz 1843

$\uparrow$ Hybodontes Agassiz 1843 Tome III: 206 (family) $\uparrow$ Hybodus Agassiz 1837 [latinized to $\uparrow$ Hybodontidae by Owen 1846; latinized to †Hybodontes by Giebel 1846: 152 and Giebel 1847: 311, latinized to $\uparrow$ Hybondontini [ $\uparrow$ Hybodontini] by Bonaparte 1850a: 453; considered valid with this authorship by Bronn 1849: 650, by Bleeker 1859: XII and by de Koninck 1878: 26 Art. 11.7.2]

Family $\uparrow$ Thaiodontidae Cuny, Suteethorn, Kamha \& Buffetaut 2008 
$†$ Thaiodontidae Cuny, Suteethorn, Kamha \& Buffetaut 2008: 98 (family) †Thaiodus Cappetta, Buffetaut \& Suteethorn 1990

Family $\uparrow$ Tristychiidae Moy-Thomas 1939

$\dagger$ Tristychiidae Moy-Thomas 1939: 65 (family) †Tristychius Agassiz 1837

Family $\uparrow$ Distobatidae Werner 1989

$\uparrow$ Distobatidae Werner 1989: 88 (family) †Distobatus Werner 1989

Family $\uparrow$ Acrodontidae Giebel 1846

$\uparrow$ Acrodini Giebel 1846: 152 (family) $\uparrow$ Acrodus Agassiz 1834 [corrected to $\uparrow$ Acrodontidae by Fraas 1896: 17]

Family $\uparrow$ Polyacrodontidae Glickman 1964

$\uparrow$ Polyacrodontidae Glickman 1964a: 10 (family) †Polyacrodus Jaekel 1889 [also Glickman 1964b: 212]

Family $\uparrow$ Lonchidiidae Herman 1977

$\dagger$ Londichiidae [†Lonchidiidae] Herman 1977: 40, 44 (family) $\dagger$ Lonchidion Estes 1964 [family name sometimes seen as $\uparrow$ Lonchidionidae]

Family $\uparrow$ Steinbachodontidae Reif 1980

$†$ Steinbachodontidae Reif 1980: 63 (family) †Steinbachodus Reif 1980

Family $\uparrow$ Pseudodalatiidae Reif 1978

$\uparrow$ Pseudodalatiidae Reif 1978: 55 (family) $\uparrow$ Pseudodalatias Reif 1978

Family $\uparrow$ Homalodontidae Mutter, Neuman \& de Blanger 2008

†Wapitiodidae Mutter, de Blanger \& Neuman 2007: 309 (family) †Wapitiodus Mutter, de Blanger \& Neuman 2007 [type genus preoccupied by $\uparrow$ Wapitiodus Orchard 2005 in Conodonta; invalid, Art. 39]

$†$ Homalodontidae Mutter, Neuman \& de Blanger 2008: 419 (family) †Homalodontus Mutter, Neuman \& de Blanger 2008

Family $\uparrow$ Sphenacanthidae Maisey 1982, name in prevailing recent practice

? †Xenosynechodontidae Glickman 1980: ? (family) †Xenosynechodus Glickman 1980 [in $? †$ Xenacanthidae, family uncertain]

$\dagger$ Sphenacanthidae Maisey 1982: 21 (family) †Sphenacanthus Agassiz 1837

\section{Infraclass ELASMOBRANCHII (Neoselachii)}

Incertae sedis:

Family $\uparrow$ Agaleidae Rees 2000

$\uparrow$ Agaleidae Rees 2000: 412 (family) $\uparrow$ Agaleus Duffin \& Ward 1983

Family $\uparrow$ Anachronistidae Duffin \& Ward 1983

$\uparrow$ Anachronistidae Duffin \& Ward 1983: 95 (family) †Anachronistes Duffin \& Ward 1983 (=

$\uparrow$ Cooleyella Gunnell 1933) [family name sometimes seen as †Cooleyellidae]

Family $\nmid$ Lugalepididae Karatajūtè-Talimaa 1997

†Lugalepididae Karatajūtè-Talimaa 1997a: 25 (family) †Lugalepis Karatajūtè-Talimaa 1997

Family $\uparrow$ Mcmurdodontidae White 1968

$†$ Mcmurdodontidae White 1968: 9 (family) $†$ Mcmurdodus White 1968

Family $†$ Ostenoselachidae Duffin 1998

†Ostenoselachidae Duffin 1998: 5 (family) †Ostenoselache Duffin 1998

\section{Superorder Galeomorphi (Selachimorpha)}

Incertae sedis:

Family $\uparrow$ Ptychodontidae Jaekel 1898

$\uparrow$ Ptychodontinae Jaekel 1898: 50 (subfamily) $\uparrow$ Ptychodus Agassiz 1838

$†$ Hylaeobatidae Herman \& van Waes 2014: 161 (family) †Hylaeobatis Woodward 1916 [not published according to the rules, not available] 


\section{Order $\uparrow$ Synechodontiformes}

Family $\uparrow$ Palaeospinacidae Regan 1906

$\uparrow$ Palaeospinacinae Regan 1906: 723, 750 (subfamily) †Palaeospinax Egerton 1872 [ $\uparrow$ Palaeospinax is a nomen dubium, no longer recognized as a valid genus]

$\uparrow$ Synechodontidae Casier 1947b: 34 (family) †Synechodus Woodward 1888

†Orthacodidae Glickman 1957: 114 (family) †Orthacodus Woodward 1889 [corrected to †Orthacodontidae by Beaumont 1960: 4]

$\uparrow$ Paraorthacodidae Glickman 1958: 668 (family) †Paraorthacodus Glickman 1957 [family name sometimes seen as $\uparrow$ Paraorthacodontidae]

Family $\uparrow$ Pseudonotidanidae Underwood \& Ward 2004

$\uparrow$ Welcommiidae Leidner \& Delsate 2000: 46 (family) †Welcommia Capetta 1990 [unavailable publication, family-group name not available]

†Welcommiidae Delsate 2001: 59 (family) †Welcommia Capetta 1990 [not published according to the rules, not available]

$\dagger$ Pseudonotidanidae Underwood \& Ward 2004: 474 (family) †Pseudonotidanus Underwood \& Ward 2004

\section{Order Heterodontiformes}

Family Heterodontidae Gray 1851

$\uparrow$ Paracestrationidae Herman \& van Waes 2012c: 11 (family) $\uparrow$ Paracestracion Koken 1911 [type genus as $\uparrow$ Paracestration, name must be corrected Art. 32.5.3; corrected to $\uparrow$ Paracestracionidae by Herman \& van Waes 2014: 171]

\section{Order Orectolobiformes}

Incertae sedis:

$\uparrow$ Palaeocarcharidae Applegate 2001a (family) †Palaeocarcharias Beaumont 1960 [unavailable publication, family-group name not available]

Family $\uparrow$ Phorcynidae Herman \& van Waes 2014

$†$ Phorcynidae Herman \& van Waes 2014: 114, 135, 173 (family) †Phorcynis Thiollière 1854 [family name sometimes seen as $\uparrow$ Phorcinidae]

\section{Suborder Parascyllioidei}

Family Parascylliidae Gill 1862

\section{Suborder Orectoloboidei}

Family Brachaeluridae Applegate 1974

Family Orectolobidae Gill 1896

Family Hemiscylliidae Gill 1862

Family Ginglymostomatidae Gill 1862

Pseudoginglymostomatidae Herman \& van Waes 2014: 118 (family) Pseudoginglymostoma

Dingerkus 1986 [not published according to the rules, not available]

Family Stegostomatidae Gill 1862

Family Rhincodontidae Müller \& Henle 1841 [ICZN Opinion 1278]

\section{Order Lamniformes}

Family $\uparrow$ Haimirichiidae Vullo, Guinot \& Barbe 2016

$\uparrow$ Haimirichiidae Vullo, Guinot \& Barbe 2016: 1004 (family) $\uparrow$ Haimirichia Vullo, Guinot \& Barbe 2016

Family †Otodontidae Glickman 1964

†Otodontidae Glickman 1964a: 103 (family) †Otodus Agassiz 1838 
Family $\uparrow$ Xiphodolamiidae Glickman 1964

$\dagger$ Xipodolamiinae [ $†$ Xiphodolamiinae] Glickman 1964a: 105 (subfamily) $†$ Xiphodolamia Leidy 1877

Family $\uparrow$ Cardabiodontidae Siverson 1999

$\uparrow$ Cardabiodontidae Siverson 1999: 49 (family) †Cardabiodon Siverson 1999

Family $\uparrow$ Cretoxyrhinidae Glickman 1958

$\uparrow$ Cretoxyrhinidae Glickman 1958: 669 (family) †Cretoxyrhina Glickman 1958

Family $\uparrow$ Archaeolamnidae Underwood \& Cumbaa 2010

$\uparrow$ Archaeolamnidae Underwood \& Cumbaa 2010: 921 (family) $\uparrow$ Archaeolamna Siverson 1992

Family †Pseudoscapanorhynchidae Herman 1979

$†$ Pseudoscapanorhynchidae Herman 1979: 361 (family) †Pseudoscapanorhynchus Herman 1977

Family $\uparrow$ Anacoracidae Casier 1947

$\uparrow$ Coracidae sometimes used for fossil fishes? (family) †Corax Agassiz 1843 [type genus preoccupied by Corax Ledru 1810 in Aves; invalid, Art. 39; not Coraciidae Rafinesque 1815 in Aves]

$\uparrow$ Anacoracidae Casier 1947a: 5 (family) †Anacorax White \& Moy-Thomas 1940 [also Casier 1947b: 36; also as a new family in Glickman 1956; senior objective synonym of $\uparrow$ Squalicoracidae Patterson 1967]

$\uparrow$ Squalicoracidae Patterson in Andrews, Gardiner, Miles \& Patterson 1967: 671 (family) $\uparrow$ Squalicorax Whitley 1939 [junior objective synonym of $\uparrow$ Anacoracidae Casier 1947, invalid, Art. 61.3.2]

$\uparrow$ Ptychocoracidae Herman \& van Waes 2012a: 8 (family) $\uparrow$ Ptychocorax Glickman \& Istchenko 1980

$†$ Paracoracidae Herman \& van Waes 2014: 186 (family) †Paracorax Cappetta 1977 [not published according to the rules, not available; also type genus preoccupied by Paracorax Lambrecht 1933 in Aves; invalid, Art. 39]

Family $\uparrow$ Pseudocoracidae Capetta 2012

†Pseudocoracidae Capetta 2012: 249 (family) †Pseudocorax Priem 1897

Family Mitsukurinidae Jordan 1898

†Scapanorhynchidae White 1936a: 4 (family) †Scapanorhynchus Woodward 1889

$\uparrow$ Anomotodontidae Herman 1979: 362 (family) †Anomotodon Arambourg 1952

Family Odontaspididae Müller \& Henle 1839 [ICZN Opinion 723]

$\dagger$ Jekelotodontidae [†Jaekelotodontidae] Glickman 1964a: 11 (family) †Jaekelotodus Menner 1928

$\dagger$ Eostriatolamiidae Glickman in Zhelezko \& Glickman 1971: 179 (family) †Eostriatolamia Glickman 1979 [no valid type genus, Art. 11.7.1.1; also name only, published after 1960, not available, Art. 13.1.1]

$\uparrow$ Striatolamiidae Glickman, Mertiniene \& Nessov in Glickman, Mertiniene, Nessov, Rozhdestvensky, Khosatzky \& Yakovlev 1987: 252 (family) †Striatolamia Glickman 1964 [name only, published after 1960, not available, Art. 13.1.1]

$\uparrow$ Striatolamidae Zhelezko 1989: 16 (family) †Striatolamia Glickman 1964 [correct stem would be Striatolami-; name only, published after 1960, not available, Art. 13.1.1]

$\dagger$ Johnlonginae Shimada, Popov, Siversson, Welton \& Long 2015: 2 (subfamily) †Johnlongia Siverson 1996 [correct stem would be Johnlongi-]

Family Carchariidae Müller \& Henle 1838 [ICZN Opinion 1459]

Family Pseudocarchariidae Taylor, Compagno \& Struhsaker 1983

Family Alopiidae Bonaparte 1835

Family Megachasmidae Taylor, Compagno \& Struhsaker 1983

Family Cetorhinidae Gill 1861 
Family Lamnidae Bonaparte 1835

$\nmid$ Lamiostomatidae Glickman 1964a: 11, 105 (family) †Lamiostoma Glickman 1964

Family $\uparrow$ Paraisuridae Herman 1979

$\uparrow$ Paraisuridae Herman 1979: 361 (family) †Paraisurus Glickman 1957

Family $†$ Serratolamnidae Landemaine 1991

$\dagger$ Serratolamnidae Landemaine 1991: 12 (family) †Serratolamna Landemaine 1991

Family $†$ Eoptolamnidae Kriwet, Klug, Canudo \& Cuenca-Bescós 2008

$†$ Eoptolamnidae Kriwet, Klug, Canudo \& Cuenca-Bescós 2008: 280 (family) †Eoptolamna Kriwet, Klug, Canudo \& Cuenca-Bescós 2008 [family name sometimes seen as $\uparrow$ Eoleptolamnidae]

\section{Order Carcharhiniformes}

Family Scyliorhinidae Gill 1862

$\uparrow$ Megascyliorhinidae Pfeil 1984: 112 (family) †Megascyliorhinus Cappetta \& Ward 1977

$†$ Premontreinae Cappetta 1992a: 644 (subfamily) $\uparrow$ Premontreia Cappetta 1992

$†$ Pteroscylliinae Cappetta 1992a: 644 (subfamily) $†$ Pteroscyllium Cappetta 1980

$\uparrow$ Palaeoscylliidae Herman \& van Waes 2012d: 50 (family) †Palaeoscyllium Wagner 1857

Family Pentanchidae Smith 1912

Holohalaeluridae Herman \& van Waes 2012d: 51 (family) Holohalaelurus Fowler 1934 [not published according to the rules, not available; Recent, omitted in Van der Laan et al. 2014]

Family Proscylliidae Fowler 1941

Family Pseudotriakidae Gill 1893

Family Leptochariidae Gray 1851

Family Triakidae Gray 1851

Family Hemigaleidae Hasse 1878

Family Carcharhinidae Jordan \& Evermann 1896

Galeocerdidae Herman \& van den Eeckhaut 2010: 51 (family) Galeocerdo Müller \& Henle 1837

[Recent, omitted in Van der Laan et al. 2014]

Triaenodonidae Herman \& van den Eeckhaut 2010: 52 (family) Triaenodon Müller \& Henle 1834 [Recent, omitted in Van der Laan et al. 2014; correct stem would be Triaenodont-]

Family Sphyrnidae Bonaparte 1840

\section{Superorder Squalomorphi}

Order Hexanchiformes

Family Chlamydoselachidae Garman 1884

Family $\uparrow$ Crassodontidanidae Kriwet \& Klug 2016

$\uparrow$ Crassonotidae Kriwet \& Klug 2011: S108 (family) †Crassodontidanus Kriwet \& Klug 2011

[no stem of the type genus, not available, Art. 11.7.1.1]

$\uparrow$ Crassodontidanidae Kriwet \& Klug 2016: 1 (family) †Crassodontidanus Kriwet \& Klug 2011

Family Hexanchidae Gray 1851

$†$ Eonotidanidae Pfeil 1983: 25 (family) †Eonotidanus Pfeil 1983

Family Heptranchidae Barnard 1925

\section{Order Squaliformes}

Family †Eoscymnidae Herman \& van den Eeckhaut 2010

$\dagger$ Eoscymnidae Herman \& van den Eeckhaut 2010: 39 (family) †Eoscymnus Herman \& van den

Eeckhaut 2010 [family name sometimes seen as $\dagger$ Eoscymnorhinidae]

Family Centrophoridae Bleeker 1859

Family Etmopteridae Fowler 1934

Aculeolidae Herman \& van Waes 2012b: 8 (family) Aculeola de Buen 1959 [Recent, omitted in

Van der Laan et al. 2014] 
Family Somniosidae Jordan 1888

Family Oxynotidae Gill 1863

Family Dalatiidae Gray 1851

Family Squalidae de Blainville 1816

\section{Order $\uparrow$ Protospinaciformes}

Family $\uparrow$ Protospinacidae Woodward 1919

$†$ Protospinacidae Woodward 1919: 232 (family) †Protospinax Woodward 1919

\section{Order Echinorhiniformes}

Family †Pseudoechinorhinidae Herman \& van Waes 2014

$\dagger$ Pseudoechinorhinidae Herman \& van Waes 2014: 166 (family) $†$ Pseudoechinorhinus Pfeil 1983

Family †Orthechinorhinidae Herman \& van Waes 2014

†Orthechinorhinidae Herman \& van Waes 2014: 167 (family) †Orthechinorhinus Adnet 2006

Family Echinorhinidae Gill 1862

\section{Order Squatiniformes}

Family $\uparrow$ Pseudorhinidae Klug \& Kriwet 2012

$\uparrow$ Pseudorhinidae Klug \& Kriwet 2012: 94 (family) †Pseudorhina Jaekel 1898

Family Squatinidae de Blainville 1816

\section{Order Pristiophoriformes}

Family Pristiophoridae Bleeker 1859

\section{Division Batomorphi}

Incertae sedis:

$\uparrow$ Archaeobatidae Delsate \& Candoni 2001: 132 (family) †Toarcibatis Delsate \& Candoni 2001 [no stem of the type genus, not available, Art. 11.7.1.1; family name sometimes seen as †Archaeobatididae]

$\uparrow$ Arthropteridae Jordan 1905: 553 (family) $\uparrow$ Arthropterus Agassiz 1843 [type genus preoccupied by Arthropterus M'Leay 1838 in Carabidae Coleoptera; invalid, Art. 39]

$\uparrow$ Asterodermini Bonaparte 1850a: 453 (subfamily) $\uparrow$ Asterodermus Agassiz 1843 [type genus inferred from the stem, Art. 11.7.1.1; family name sometimes seen as $\uparrow$ Asterodermatidae and $\uparrow$ Astrodermidae]

$\dagger$ Belemnobatidae Suteethorn, Le Loeuff, Buffetaut, Suteethorn \& Wongko 2013: 461 (family) $\uparrow$ Belemnobatis Thiollière 1854 [not published according to the rules, not available; family name sometimes seen as $\dagger$ Belemnobatididae]

Centrobatidae Jaekel 1898: 51 [ref. 34311] (family) ? [no valid type genus, not available; Recent, omitted in Van der Laan et al. 2014]

Rhinorajidae Jaekel 1898: 51 (family) ? [not based on Rhinoraja; no valid type genus, not available; Recent, omitted in Van der Laan et al. 2014]

$\dagger$ Spathobatidae Dames 1888: 130 (family) †Spathobatis Thiollière 1854 [type genus inferred from the stem, Art. 11.7.1.1]

\section{Order Torpediniformes}

Family Torpedinidae Henle 1834

Family Hypnidae Gill 1862

Family Narcinidae Gill 1862

Family Narkidae Fowler 1934

\section{Order Rajiformes}

Family $\uparrow$ Cyclobatidae Jaekel 1911 
$\uparrow$ Cyclobatidae Jaekel 1911: 64 (family) †Cyclobatis Egerton 1844 [family name sometimes seen as $\dagger$ Cyclobatididae]

Family $\uparrow$ Hypsobatidae Cappetta 1992

$\uparrow$ Hypsobatidae Cappetta 1992b: 33 (family) $\uparrow$ Hypsobatis Cappetta 1992 [family name sometimes seen as $\uparrow$ Hypsobatididae]

Family $\uparrow$ Parapalaeobatidae Cappetta 1992

$\uparrow$ Parapalaeobatidae Cappetta 1992b: 50 (family) †Parapalaeobates Weiler 1930

Family Rajidae de Blainville 1816

Rostrorajinae Ishihara, Treloar, Bor, Senou \& Jeong 2012: 31 (subfamily) Rostroraja [not published according to the rules, not available; Recent, omitted in Van der Laan et al. 2014]

Family Anacanthobatidae von Bonde \& Swart 1923

Family Crurirajidae Hulley 1972

Family Arhynchobatidae Fowler 1934

Family Gurgesiellidae Buen 1959

\section{Order Rhinopristiformes}

Family Trygonorrhinidae Last, Séret \& Naylor 2016

Family Rhinobatidae Bonaparte 1835

Family Rhinidae Müller \& Henle 1841

Family Glaucostegidae Last, Séret \& Naylor 2016

Family Pristidae Bonaparte 1835

\section{Order $\uparrow$ Sclerorhynchiformes}

Family $\uparrow$ Sclerorhynchidae Cappetta 1974, name in prevailing recent practice $\dagger$ Ischyrhizidae Cope 1875: 280 (family) †Ischyrhiza Leidy 1856

$\uparrow$ Ganopristinae Arambourg 1941: 139 (subfamily) †Ganopristis Arambourg 1935 [sometimes seen as $\uparrow$ Ganopristiinae]

$\uparrow$ Sclerorhynchidae Cappetta 1974: 225 (family) †Sclerorhynchus Woodward 1889

$\uparrow$ Schizorhizinae Kirkland \& Aguillón-Martínez 2002: 21 (subfamily) †Schizorhiza Weiler 1930

Family $\uparrow$ Ptychotrygonidae Kriwet, Nunn \& Klug 2009

$\dagger$ Ptychotrygonidae Kriwet, Nunn \& Klug 2009: 333 (family) †Ptychotrygon Jaekel 1894 [also as a new family in Herman \& van Waes 2012a: 4]

\section{Order Myliobatiformes}

Suborder Platyrhinoidei

Family Platyrhinidae Jordan 1923

\section{Suborder Myliobatoidei}

Family $\uparrow$ Rhombodontidae Cappetta 1987

$\uparrow$ Rhombodontidae Cappetta 1987: 174 (family) $\uparrow$ Rhombodus Dames 1881

Family Zanobatidae Fowler 1934

Family Hexatrygonidae Heemstra \& Smith 1980

Family Dasyatidae Jordan \& Gilbert 1879

Family Potamotrygonidae Garman 1877

Family Urotrygonidae McEachran, Dunn \& Miyake 1996

Family Gymnuridae Fowler 1934

Family Plesiobatidae Nishida 1990

Family Urolophidae Müller \& Henle 1841

Family Aetobatidae Agassiz 1858

Family Myliobatidae Bonaparte 1835

$\dagger$ Hypolophitinae White 1935a: 34 (subfamily) $\dagger$ Hypolophites Stromer 1910 
$\dagger$ Rhizochlatridae Silva Santos \& Travassos 1960: 16 (family) $†$ Rhizochlatrus Silva Santos \& Travassos 1960

$\uparrow$ Brachyrhizodontidae Herman \& van Waes 2012a: 10 (family) †Brachyrhizodus Romer 1942

Family Rhinopteridae Jordan \& Evermann 1896

$\nmid$ Zygzabatidae Mendiola 1995: 2 (family) †Zygzabatis Mendiola 1995

Family Mobulidae Gill 1893

CLASS OSTEICHTHYES (Euteleostomi)

Incertae sedis:

$\uparrow$ Andreolepididae Märss 2001: 179 (family) †Andreolepis Gross 1968

$\uparrow$ Halecopsidae Casier 1946: 114 (family) $†$ Halecopsis Woodward 1901 [family name sometimes seen as $†$ Halecopsididae]

$\uparrow$ Histiothrissinae Arambourg 1955: 40 (subfamily) $\uparrow$ Histiothrissa Woodward 1901

$\uparrow$ Lophosteidae Gross 1969: 17 (family) †Lophosteus Pander 1856

$\uparrow$ Mesolepidae Young 1866: 316 (family) †Mesolepis Young 1866 [family name sometimes seen as $†$ Mesolepididae]

\section{Order $\dagger$ Dialipiniformes}

$\uparrow$ Dialipina Schultze 1968

\section{Subclass ACTINOPTERYGII}

Incertae sedis:

$\dagger$ Coccolepidae Berg 1940: 400 (family) †Coccolepis Agassiz 1844 [family name also seen as $\dagger$ Coccolepididae]

$\uparrow$ Tienshaniscidae Liu \& Wang 1978: 3 (family) †Tienshaniscus Liu \& Wang 1978 [Lu \& Chen 2010: 7 wrote $\uparrow$ Tienshanididae]

$\dagger$ †ighuroniscidae Su 1985: 65, 129 (family) †Uighuroniscus Su 1985

\section{Order $\uparrow$ Cheirolepidiformes}

Family $\uparrow$ Acropholidae Kazantseva-Selezneva 1977

$\uparrow$ Acropholidae Kazantseva-Selezneva 1977: 102 (family) $\uparrow$ Acropholis Aldinger 1935 [family name sometimes seen as $\uparrow$ Acropholididae]

Family $\uparrow$ Cheirolepididae Pander 1860, spelling in prevailing recent practice

$\uparrow$ Cheirolepiden Pander 1860: 69 (family) †Cheirolepis Agassiz 1835 [family name sometimes seen as $\uparrow$ Cheirolépidés Eichwald 1860: 1572; latinized to $\uparrow$ Cheirolepini by Huxley 1861: 40 and by Traquair 1875: 238 , latinized to $\dagger$ Chirolepidae by Hoernes 1884: 438 ; considered valid with this authorship by Huxley 1861: 40, by Traquair 1875: 238, by Berg, Kazantseva \& Obruchev 1964: 336, by Andrews et al. 1967: 644, by Kazantseva-Selezneva 1977: 99, by Beltan 1990: 79 and by Gardiner 1993b: 611 Art. 11.7.2; family name also seen as $†$ Cheirolepididae]

Family †Karaunguriidae Kazantseva-Selezneva 1977

$\dagger$ Karaunguriidae Kazantseva-Selezneva 1977: 102 (family) †Karaunguria KazantsevaSelezneva 1977

\section{Order $\uparrow$ Palaeonisciformes}

Incertae sedis:

Family $\uparrow$ Acrolepididae Aldinger 1937, spelling in prevailing recent practice

$\uparrow$ Acrolepidae Aldinger 1937: 32, 250 (family) †Acrolepis Agassiz 1833 [family name also seen as $\uparrow$ Acrolepididae]

Family $\uparrow$ Asarotidae Schaeffer 1968

$\uparrow$ Asarotidae Schaeffer 1968: 4 (family) †Asarotus Schaeffer 1968

Family $\uparrow$ Atherstoniidae Gardiner 1967

$\uparrow$ Atherstoniidae Gardiner 1967: 176 (family) †Atherstonia Woodward 1889 
Family $\dagger$ Birgeriidae Aldinger 1937

? †Xenesthidae Jordan 1923: 160 (family) $†$ Xenesthes Jordan 1907 [family uncertain]

$\dagger$ Birgeriidae Aldinger 1937: 377 (family) †Birgeria Stensiö 1919 [name only, but used as valid by Berg 1940: 172, by Romer 1945: 579, by Schultz 1948: 228 and by Andrews et al. 1967: 645 Art. 13.2.1]

Family $\uparrow$ Boreolepididae Aldinger 1937, spelling in prevailing recent practice

$\uparrow$ Boreolepidae Aldinger 1937: 122, 304 (family) †Boreolepis Aldinger 1937 [family name also seen as $\uparrow$ Boreolepididae]

Family $\uparrow$ Brazilichthyidae Cox \& Hutchinson 1991

$\dagger$ Brazilichthyidae Cox \& Hutchinson 1991: 563 (family) †Brazilichthys Cox \& Hutchinson 1991

Family $\uparrow$ Canobiidae Aldinger 1937

$\uparrow$ Canobiidae Aldinger 1937: 317 (family) †Canobius Traquair 1881

Family $\dagger$ Carbovelidae Romer 1945

$\dagger$ Carbovelidae Romer 1945: 579 (family) †Carbovelus White 1927 [name only, but used as valid by Schultz 1948: 229, by Romer 1966: 352, by Andrews et al. 1967: 645 and by Gardiner 1993b: 614 Art. 13.2.1]

Family $\uparrow$ Centrolepididae Gardiner 1960, spelling in prevailing recent practice

$\dagger$ Centrolepididae Gardiner 1960: 248 (family) †Centrolepis Egerton 1858 [family name also seen as $\dagger$ Centrolepidae]

Family $\dagger$ Coccocephalichthyidae Fowler 1951 (1945), name in prevailing recent practice, Art. 40.2

$\uparrow$ Cocconiscidae Romer 1945: 579 (family) †Cocconiscus White \& Moy-Thomas 1940 [name only, but used as valid by Schultz 1948: 228 Art. 13.2.1; senior objective synonym of $\dagger$ Coccocephalichthyidae Fowler 1951]

$†$ Coccocephalichthyidae Fowler 1951: 2 (family) †Coccocephalichthys Whitley 1940 [type genus inferred from the stem, Art. 11.7.1.1; junior objective synonym $\uparrow$ Cocconiscidae Romer 1945, invalid, Art. 61.3.2, but name in prevailing recent practice Art. 40.2; family name sometimes seen as $†$ Coccocephalichthidae]

$\uparrow$ Monesedeiphidae Beltan 1990: 79 (family) $\uparrow$ Monesedeiphus Beltan 1990

$\dagger$ Irajapintoseidae Beltan 1990: 81 (family) †Irajapintoseidon Beltan 1990 [correct stem is Irajapintoseid-; original, spelling a printing error?]

Family $†$ Commentryidae Gardiner 1963

†Commentryidae Gardiner 1963: 290 (family) †Commentrya Sauvage 1888

Family †Cornuboniscidae White 1939

†Cornuboniscidae White 1939: 42 (family) †Cornuboniscus White 1939

Family $\uparrow$ Cosmolepididae Gardiner 1967

$\uparrow$ Cosmolepididae Gardiner 1967: 177 (family) †Cosmolepis Egerton 1858 [family name sometimes seen as $\uparrow$ Cosmolepidae]

Family $\uparrow$ Cosmoptygiidae Gardiner 1963

†Cosmoptygiidae Gardiner 1963: 259 (family) †Cosmoptygius Traquair 1877 [family name sometimes seen as $\dagger$ Cosmoptygidae]

Family $\uparrow$ Cryphiolepididae Moy-Thomas 1939, spelling in prevailing recent practice

$\dagger$ Cryphiolepidae Moy-Thomas 1939: 112 (family) †Cryphiolepis Traquair 1881 [family name also seen as $\dagger$ Cryphiolepididae]

Family $\uparrow$ Dicellopygidae Romer 1945

†Dicellopygidae Romer 1945: 579 (family) †Dicellopyge Brough 1931 [name only, but used as valid by Schultz 1948: 228, by Romer 1966: 352, by Andrews et al. 1967: 647, by Fowler 1971c: 381 and by Kazantseva-Selezneva 1977: 107 Art. 13.2.1]

Family $†$ Eigiliidae Kazantseva-Selezneva 1977

$\dagger$ Eigiliidae Kazantseva-Selezneva 1977: 112 (family) †Eigilia Kazantseva-Selezneva 1977 [family name sometimes seen as $†$ Eigillidae] 
Family †Elonichthyidae Aldinger 1937

$\uparrow$ Elonichthyidae Aldinger 1937: 16, 204 (family) †Elonichthys Giebel 1848

Family $\dagger$ Eurynotoididae Minikh \& Minikh 1990

$\dagger$ Eurynotoidiidae [ $†$ Eurynotoididae] Minikh \& Minikh 1990: 86 (family) $†$ Eurynotoides Berg 1940

Family $\uparrow$ Gyrolepidotidae Gardiner 1967

$\uparrow$ Gyrolepidotidae Gardiner 1967: 175 (family) †Gyrolepidotus Rohon 1889

Family $\uparrow$ Holuridae Moy-Thomas 1939

$\dagger$ Holuridae Moy-Thomas 1939: 113 (family) $†$ Holurus Traquair 1881

Family †Lawniidae Gardiner 1967

$\dagger$ Lawniidae Gardiner 1967: 176 (family) †Lawnia Wilson 1953

Family $\uparrow$ Pygopteridae Fitzinger 1873

$\uparrow$ Pygopteri Fitzinger 1873: 52 (family) $\uparrow$ Pygopterus Agassiz 1833

Family $\uparrow$ Sceletophoridae Štamberg 2006

$\dagger$ Sceletophoridae Štamberg 2006: 217 (family) †Sceletophorus Frič 1894

Family $\uparrow$ Strepheoschemidae Gardiner 1985

$\uparrow$ Strepheoschemidae Gardiner 1985: 61 (family) †Strepheoschema White 1927 [family name sometimes seen as $†$ Strepheoschematidae]

Family $\dagger$ Trissolepididae Frič 1893, spelling in prevailing recent practice

$\dagger$ Trissolepidae Frič 1893: 76 (family) †Trissolepis Frič 1893 [senior objective synonym of $\dagger$ Sphaerolepidae Fowler 1951; family name also seen as $†$ Trissolepididae]

$\uparrow$ Gymnoniscidae Berg 1936: 345 (family) †Gymnoniscus Berg 1936

$\dagger$ Sphaerolepidae Fowler 1951: 2 (family) †Sphaerolepis Frič 1877 [name only; junior objective synonym of $\uparrow$ Trissolepidae Frič 1893, invalid, Art. 61.3.2; family name sometimes seen as $\uparrow$ Sphaerolepididae]

Family †Urosthenidae Woodward 1931

†Urosthenidae Woodward 1931: 366 (family) †Urosthenes Dana 1848 [family name sometimes seen as $\dagger$ Urostheneidae]

Family $\uparrow$ Varialepididae Minikh 1990

†Varialepididae Minikh 1990: 71 (family) †Varialepis Minikh 1986

Family $\uparrow$ Whiteichthyidae Kazantseva-Selezneva 1977

†Whiteichthyidae Kazantseva-Selezneva 1977: 108 (family) †Whiteichthys Moy-Thomas 1942

Family $\uparrow$ Stegotrachelidae Gardiner 1963, name in prevailing recent practice

$\dagger$ Tegeolepidae Romer 1945: 579 (family) $†$ Tegeolepis Miller 1892 [name only, but used as valid by Schultz 1948: 228, by Gardiner 1963: 299, by Romer 1966: 352, by Andrews et al. 1967: 645, by Moy-Thomas \& Miles 1971: 92, by Fowler 1971c: 383 and by Gardiner 1993b: 612 Art. 13.2.1; family name also seen as $\dagger$ Tegeolepididae]

$\uparrow$ Stegotrachelidae Gardiner 1963: 294 (family) †Stegotrachelus Woodward \& White 1926

Family $\uparrow$ Kentuckiidae

$\dagger$ Kentuckiidae Gardiner 1993b: 612 (family) †Kentuckia Rayner 1951 [name only, published after 1960, not available, Art. 13.1.1]

Family $\uparrow$ Howqualepididae Long, Choo \& Young 2008

$\dagger$ Howqualepididae Long, Choo \& Young 2008: 396 (family) †Howqualepis Long 1988

Family $\uparrow$ Moythomasiidae Kazantseva 1971

$\uparrow$ Moythomasiidae Kazantseva 1971: 164 (family) $\uparrow$ Moythomasia Gross 1950

† Mimiidae Gardiner 1993b: 611 (family) †Mimia Gardiner \& Bartram 1977 [name only, published after 1960, not available, Art. 13.1.1; also type genus preoccupied by Mimia Evans 1953 in Lepidoptera; invalid, Art. 39; type genus replaced by $\uparrow$ Mimipiscis Choo 2012, if found to belong to a separate family, a new family-group name is needed]

Family $\uparrow$ Rhabdolepididae Gardiner 1963, spelling in prevailing recent practice 
$\dagger$ Rhabdolepididae Gardiner 1963: 284 (family) $†$ Rhabdolepis Troschel 1857 [family name also seen as $\uparrow$ Rhabdolepidae]

†Osorioichthyidae Gardiner 1967: 175 (family) †Osorioichthys Casier 1954

$\dagger$ Willomorichthyidae Gardiner 1969: 423, 438 (family) †Willomorichthys Gardiner 1969

Family $\uparrow$ Aesopichthyidae Poplin \& Lund 2000

$\uparrow$ Aesopichthyidae Poplin \& Lund 2000: 429 (family) †Aesopichthys Poplin \& Lund 2000

Family $\uparrow$ Amblypteridae Romer 1945

$\dagger$ Heterocercoidei Bleeker 1859: XIV (family) $\uparrow$ Amblypterus Agassiz 1833 [no stem of the type genus, not available, Art. 11.7.1.1]

†Amblypteridae Romer 1945: 579 (family) †Amblypterus Agassiz 1833 [name only, but used as valid by Schultz 1948: 228, by Gardiner 1963: 290, by Romer 1966: 352, by Andrews et al. 1967: 646, by Fowler 1971c: 376, by Kazantseva-Selezneva 1977: 107 and by Gardiner 1993b: 614 Art. 13.2.1]

Family $\uparrow$ Paramblypteridae Blot 1966

$†$ Paramblypteridae Blot 1966: 15 (family) $†$ Paramblypterus Sauvage 1888

Family $\uparrow$ Rhadinichthyidae Romer 1945

$\uparrow$ Rhadinichthyidae Romer 1945: 579 (family) †Rhadinichthys Traquair 1877 [name only, but used as valid by Schultz 1948: 228, by Romer 1966: 352, by Andrews et al. 1967: 645, by Fowler 1971b: 40, by Kazantseva-Selezneva 1977: 107 and by Gardiner 1993b: 613 Art. 13.2.1]

Family $\uparrow$ Palaeoniscidae Bonaparte 1846

$\dagger$ Palaeoniscini Bonaparte 1846: 4 (subfamily) $†$ Palaeoniscum de Blainville 1818 [type genus inferred from the stem, Art. 11.7.1.1]

$\dagger$ Oxygnathidae Berg 1940: 400 (family) $†$ Oxygnathus Egerton 1854 [type genus preoccupied by Oxygnathus Dejean 1826 in Carabidae Coleoptera; invalid, Art. 39]

$\dagger$ Thrissonotidae Berg 1955: 121 (family) †Thrissonotus Agassiz 1844

$\dagger$ Turseoidae Bock 1959: 4 (family) †Turseodus Leidy 1857 [family name also seen as $\dagger$ Turseodidae; correct stem is Turseodont-]

Family $\uparrow$ Gonatodidae Gardiner 1967, spelling in prevailing recent practice

†Gonatodidae Gardiner 1967: 146 (family) †Gonatodus Traquair 1877 [family name also seen as $\uparrow$ Gonatodontidae]

\section{Order $\dagger$ Dorypteriformes}

Family $\uparrow$ Dorypteridae Fitzinger 1873

$\uparrow$ Doryopteri Fitzinger 1873: 51 (family) $\uparrow$ Dorypterus Germar 1842 [type genus as $\uparrow$ Doryopterus, name must be corrected Art. 32.5.3; corrected to $\uparrow$ Dorypteridae by Cope 1877a: 58, confirmed by Steinmann \& Döderlein 1890: 567]

\section{Order $\uparrow$ Platysomiformes}

Family $\uparrow$ Platysomidae Bleeker 1859

$\uparrow$ Platysomatoidei Bleeker 1859: XVII (family) †Platysomus Agassiz 1833 [not Platysomatidae Bickhardt 1914 in Diptera; family name corrected to $†$ Platysomidae by Young 1866: 316 and confirmed by Günther 1880: 370; family name corrected to $\uparrow$ Platysomidae or not by Nicholson 1879: x, 979 and erratum slip in Volume II for p. 134 and 138; senior objective synonym of $\dagger$ Stroteidae Fowler 1951]

$\dagger$ Eurysomidae Young 1866: 316 (family) †Eurysomus Agassiz 1833

$\uparrow$ Scroteidae [ $†$ Stroteidae] Jordan 1923: 112 (family) $†$ Strotes Gistel 1848 [name in synonymy, not available; or, if treated as available with the help of Art. 11.6.1, junior objective synonym of $\uparrow$ Platysomatoidei Bleeker 1859, invalid, Art. 61.3.2] 
$\dagger$ Cheirodidae Moy-Thomas 1939: 126 (family) ? †Cheirodopsis Traquair 1881 or ? †Chirodus M'Coy 1848 [name only, used as valid before 2000?; not available]

$\uparrow$ Stroteidae Fowler 1951: 2 (family) †Strotes Gistel 1848 [name only; junior objective synonym of $\uparrow$ Platysomatoidei Bleeker 1859, invalid, Art. 61.3.2]

†Uropterygidae Fowler 1958: 3 (family) †Uropteryx Agassiz 1835

$\uparrow$ Chirodontidae Moy-Thomas \& Miles 1971: 94 (family) †Chirodus M’Coy 1848 [family name sometimes seen as $\uparrow$ Cheirodontidae, this spelling preoccupied by Cheirodontinae Eigenmann 1915 in fishes]

Family $†$ Bobasatraniidae Stensiö 1932

$\uparrow$ Bobasatraniidae Stensiö in Koch 1931: 74 (family) $†$ Bobasatrania White 1932 [no valid type genus, not available, Art. 11.7.1.1]

$\uparrow$ Bobasatraniidae Stensiö 1932a: 96, 125 (family) †Bobasatrania White 1932

\section{Order $\uparrow$ Tarrasiiformes}

Family $\uparrow$ Tarrasiidae Traquair 1881

$\uparrow$ Tarrasiidae Traquair 1881a: 61 (family) †Tarrasius Traquair 1881 [family name sometimes seen as $\uparrow$ Tarrassiidae]

\section{Order $\uparrow$ Guildayichthyiformes}

Family $\uparrow$ Guildayichthyidae Lund 2000

$†$ Guildayichthyidae Lund 2000: 173 (family) †Guildayichthys Lund 2000

\section{Order $\uparrow$ Phanerorhynchiformes}

Family $†$ Phanerorhynchidae Stensiö 1931

$†$ Phanerorhynchidae Stensiö in Koch 1931: 74 (family) †Phanerorhynchus Gill 1923 [type genus inferred from the stem; name only, but used as valid by Stensiö 1932a: 74, 97, by Berg 1940: 402, by Romer 1966: 353 and by Andrews et al. 1967: 648 Art. 13.2.1]

\section{Order $\uparrow$ Saurichthyiformes}

Family $†$ Saurichthyidae Bleeker 1859

$\dagger$ Saurichthyoidei Bleeker 1859: XVI (family) †Saurichthys Agassiz 1834

$\uparrow$ Rhynchodontidae Zittel 1887: 219 (family) ? [not $\uparrow$ Rhynchodus Newberry 1873] [no stem of the type genus, not available, Art. 11.7.1.1]

$\uparrow$ Belonorhynchidae Woodward 1889c: 407 (family) $†$ Belonorhynchus Bronn 1858 [type genus inferred from the stem, Art. 11.7.1.1; family name sometimes seen as $†$ Belonorynchidae]

$†$ Saurorhynchidae Jordan 1905: 17 (family) †Saurorhynchus Reis 1892

Family $\uparrow$ Yelangichthyidae Wu, Chang, Sun \& Xu 2013

$\dagger$ Yelangichthyidae Wu, Chang, Sun \& Xu 2013: 2 (family) †Yelangichthys Wu et al. 2013

\section{Order $\uparrow$ Redfieldiiformes}

Family $\uparrow$ Redfieldiidae Berg 1940, name in prevailing recent practice

$†$ Catopteridae Woodward 1890a: 424 (family) †Catopterus Redfield 1837 [also Woodward 1890b: 15; type genus preoccupied by $\uparrow$ Catopterus Agassiz 1833 in fishes; invalid, Art. 39]

$\uparrow$ Dictyopygidae Hay 1899: 789 (family) †Dictyopyge Egerton 1847 [family name sometimes seen as $\dagger$ Dictypygidae]

$\uparrow$ Redfieldiidae Berg 1940: 404 (family) $\uparrow$ Redfieldius Hay 1899

$\uparrow$ Brookvaliidae Berg 1940: 404 (family) †Brookvalia Wade 1933

$\uparrow$ Schizurichthyidae Hutchinson 1973: 285 (family) †Schizurichthys Wade 1935

Family $\uparrow$ Igornichthyidae Heyler 1977

†Igornichthyidae Heyler 1977: 13 (family) †Igornichthys Heyler 1969 


\section{Order $\uparrow$ Ptycholepidiformes}

Family $\uparrow$ Ptycholepididae Brough 1939, spelling in prevailing recent practice

$\dagger$ Ptycholepidae Brough 1939: 57 (family) $†$ Ptycholepis Agassiz 1832 [family name also seen as $\dagger$ Ptycholepididae]

$\uparrow$ Boreosomidae Gardiner 1967: 177 (family) †Boreosomus Stensiö 1921

Family $\uparrow$ Chungkingichthyidae Su 1974

$\dagger$ Chungkingichthyidae Su 1974: 1, 14 (family) †Chungkingichthys Su 1974

\section{Infraclass CLADISTIA}

Order Polypteriformes

Family Polypteridae Bonaparte 1835

\section{Infraclass ACTINOPTERI}

Incertae sedis:

$\uparrow$ Dwykiidae Gardiner 1969: 423, 443 (family) †Dwykia Gardiner 1969

$\dagger$ Inichthyidae Kazantseva-Selezneva 1981: 579 (family) †Inichthys Kazantseva-Selezneva 1979

[English edition 1982: 7]

$\dagger$ Kenderlichthyidae Kazantseva-Selezneva 1977: 106 (family) †Kenderlichthys Kazantseva-Selezneva 1977

$\uparrow$ Nematoptychiidae Kazantseva-Selezneva 1977: 104 (family) $\uparrow$ Nematoptychius Traquair 1875

$\uparrow$ Palaeobergiidae Kazantseva 1968: 104 (family) $\uparrow$ Palaeobergia Matveeva 1958

\section{Order $\uparrow$ Paphosisciformes}

Family $\uparrow$ Paphosiscidae Grogan \& Lund 2015

$†$ Paphosiscidae Grogan \& Lund 2015: 112 (family) †Paphosiscus Grogan \& Lund 2015

\section{Order $\uparrow$ Discordichthyiformes}

Family $\uparrow$ Discordichthyidae Minikh 1998

$\dagger$ Discordichthyidae Minikh 1998: 49 (family) $†$ Discordichthys Minikh 1998

\section{Order $\uparrow$ Amphicentriformes}

Family $\uparrow$ Teleopterinidae Berg 1936

$\dagger$ Teleopterinidae Berg 1936: 346 (family) †Teleopterina Berg 1936

$\dagger$ Eurylepidae Moy-Thomas 1939: 112 (family) †Eurylepis Newberry 1857 [type genus preoccupied by Eurylepis Blyth 1854 in Reptilia; invalid, Art. 39]

Family $\uparrow$ Styracopteridae Moy-Thomas 1937

$\uparrow$ Styracopteridae Moy-Thomas 1937: 353 (family) †Styracopterus Traquair 1890

$†$ Chichiidae Lu \& Chen 2010: 7 (family) †Chichia Liu \& Wang 1978 [name only?]

Family $\uparrow$ Amphicentridae Young 1866

$\uparrow$ Amphicentridae Young 1866: 316 (family) †Amphicentrum Young 1866

\section{Order $\uparrow$ Scanilepidiformes}

Family $\uparrow$ Scanilepididae Aldinger 1937, spelling in prevailing recent practice

$\uparrow$ Scanilepidae Aldinger 1937: 220 (family) †Scanilepis Aldinger 1937 [family name also seen as $\dagger$ Scanilepididae]

Family $\uparrow$ Evenkiidae Selezneva 1985

$\dagger$ Evenkiidae Selezneva 1985: 71 (family) $\dagger$ Evenkia Berg 1941 [not Evenkidae based on $\uparrow$ Evenka Rasnitsyn 1983 in Protoptera Insecta]

\section{Order $\uparrow$ Aeduelliformes}

Family $\uparrow$ Aeduellidae Romer 1945 
†Aeduellidae Romer 1945: 579 (family) †Aeduella Westoll 1937 [name only, but used as valid by Schultz 1948: 228, by Romer 1966: 352, by Andrews et al. 1967: 645, by Heyler 1969: 97, by Moy-Thomas \& Miles 1971: 93, by Fowler 1971c: 379, by Kazantseva-Selezneva 1977: 107 and by Gardiner 1993b: 614 Art. 13.2.1]

$\uparrow$ Platysellidae Heyler \& Poplin 1983: 41 (family) †Platysella Heyler \& Poplin 1983

Family $\dagger$ Haplolepidae Westoll 1944, spelling in prevailing recent practice

$\dagger$ Haplolepidae Westoll 1944: 63 (family) $†$ Haplolepis Miller 1892 [family name also seen as $\dagger$ Haplolepididae]

\section{Superdivision CHONDROSTEI \\ Order $\uparrow$ Chondrosteiformes}

Family $\uparrow$ Chondrosteidae Huxley 1861

$\uparrow$ Gyrosteini Bonaparte 1850b (subfamily) †Gyrosteus Agassiz or Morris 1854 [type genus inferred from the stem; no valid type genus, not available, Art. 11.7.1.1]

$\uparrow$ Chondrosteidae Huxley 1861: 40 (family) †Chondrosteus Egerton 1858

Family $\uparrow$ Errolichthyidae Lehman 1952

$\dagger$ Errolichthyidae Lehman 1952: 122 (family) †Errolichthys Lehman 1952

\section{Order Acipenseriformes}

Suborder $\uparrow$ Peipiaosteoidei

Family $\uparrow$ Peipiaosteidae Liu \& Zhou 1965

$†$ Peipiaosteidae Liu \& Zhou 1965: 237, 244 (family) $†$ Peipiaosteus Liu \& Zhou 1965

$\uparrow$ Spherosteinae Grande \& Bemis 1996: 112 (subfamily) †Spherosteus Jakovlev 1977

$\dagger$ Stichopterinae Grande \& Bemis 1996: 112 (subfamily) †Stichopterus Reis 1910

\section{Suborder Acipenseroidei}

Family Polyodontidae Bonaparte 1835

$†$ Paleopsephurinae Grande \& Bemis 1991: 113 (subfamily) †Paleopsephurus MacAlpin 1941

†Protopsephurinae Grande \& Bemis 1996: 112 (subfamily) †Protopsephurus Lu 1994

Family Acipenseridae Bonaparte 1831

Sturionidae Owen 1840: 68 (family) Sturio Rafinesque 1810 [junior objective synonym of Acipenseridae Bonaparte 1831, invalid, Article 61.3.2; Recent, omitted in Van der Laan et al. 2014]

$\uparrow$ Psammorhynchinae Grande \& Hilton 2006: 5 (subfamily) †Psammorhynchus Grande \& Hilton 2006 [type genus preoccupied by Psammorhynchus Meixner in Turbellaria Platyhelminthes; invalid, art. 39]

$†$ Priscosturioninae Grande \& Hilton 2009: 318 (subfamily) †Priscosturion Grande \& Hilton 2009

Pseudoscaphirhynchinae Hilton, Grande \& Bemis 2011: 157 [ref. 31407] (subfamily) Pseudoscaphirhynchus Nikolskii 1900 [Recent, omitted in Van der Laan et al. 2014]

\section{Superdivision NEOPTERYGII}

Incertae sedis:

Family $\dagger$ Lombardinidae Saint-Seine 1955

$\dagger$ Lombardinidae Saint-Seine 1955: 73 (family) †Lombardina Saint-Seine 1955

Family $†$ Signeuxellidae Saint-Seine 1955

$†$ Signeuxellidae Saint-Seine 1955: 74 (family) †Signeuxella Saint-Seine 1955 [family name sometimes seen as $\dagger$ Signeusellidae]

Family $\uparrow$ Sphaerodontidae Giebel 1846

$\dagger$ Sphaerodini Giebel 1846: 294 (family) $†$ Sphaerodus Agassiz 1844 [correct stem is Sphaerodont-; family name also seen as $†$ Sphaerodontes] 


\section{Order $\dagger$ Pycnodontiformes}

Suborder $\uparrow$ Gyrodontoidei

Family $\uparrow$ Mesturidae Nursall 1996

$\dagger$ Mesturidae Nursall 1996: 134 (family) †Mesturus Wagner 1862

Family $\uparrow$ Gyrodontidae Berg 1940

$\uparrow$ Gyrodontidae Berg 1940: 413 (family) †Gyrodus Agassiz 1833

\section{Suborder $\uparrow$ Pycnodontoidei}

Family $\uparrow$ Gibbodontidae Nursall 1999

$\dagger$ Gibbodontidae Nursall 1999: 189, 199, 210 (family) †Gibbodon Tintori 1981

Family $\uparrow$ Brembodontidae Tintori 1981

$\uparrow$ Brembodontidae Tintori 1981: 796 (family) †Brembodus Tintori 1981 [family name sometimes seen as $\dagger$ Brembodidae]

Family $\uparrow$ Macromesodontidae Nursall \& Maisey 1991

$\uparrow$ Macromesodontidae Nursall \& Maisey in Maisey 1991: 130 (family) †Macromesodon Blake 1905

Family $\uparrow$ Coccodontidae Berg 1940

†Coccodontidae Berg 1940: 413 (family) †Coccodus Pictet 1850

$\uparrow$ Trewavasiidae Nursall 1996: 140 (family) †Trewavasia White \& Moy-Thomas 1941

Family $\uparrow$ Pycnodontidae Agassiz 1833

$\uparrow$ Pycnodontae Agassiz 1832: 141 (family) $†$ Pycnodus Agassiz 1833 [no valid type genus, not available, Art. 11.7.1.1]

$†$ Pycnodontes Agassiz 1833 Tome I: 2, 15 (family) †Pycnodus Agassiz 1833 [latinized to $\dagger$ Pycnodontidae by Bonaparte 1845: 387, by Owen 1846: 50 and by Günther 1880: 366, latinized to $\uparrow$ Pycnodontes by Giebel 1846: 156 and Giebel 1847: 158, latinized to $\uparrow$ Pycnodonti by M'Coy in Sedgwick \& M'Coy 1855: 579; considered valid with this authorship by Müller 1846: 151, by Bronn 1849: 665, by Cope 1877a: 57, by Quenstedt 1885: 325, by Gardiner 1993b: 618 and by Kriwet 1999: 217 Art. 11.7.2]

$†$ Palaeobalistidae Blot 1987: 87 (family) $†$ Palaeobalistum de Blainville 1818

$\uparrow$ Nursalliidae Blot 1987: 119 (family) $\uparrow$ Nursallia Blot 1987 [family name sometimes seen as $\uparrow$ Nursallidae; correct stem is Nursalli-]

$\uparrow$ Tepexichthyinae Applegate 2001b: 3 (subfamily) †Tepexichthys Applegate 1992 [unavailable publication, family-group name not available]

†Nahuaichthyinae Applegate 2001b: 3 (subfamily) ? [unavailable publication, family-group name not available]

†Huehueichthyinae Applegate 2001b: 3 (subfamily) ? [unavailable publication, family-group name not available]

$†$ Matzichthyinae Applegate 2001b: 3 (subfamily) ? [unavailable publication, family-group name not available]

$\uparrow$ Proscinetinae Poyato-Ariza \& Wenz 2002: 221 (subfamily) †Proscinetes Gistel 1848 [curiously as "new rank"; subfamily name also seen as †Proscinitinae]

$\uparrow$ Turboscinetinae Ebert 2016: 18 (subfamily) ? †Turboscinetes Ebert 2016 [incorrectly (?) cited with type genus $\uparrow$ Proscinetes Gistel 1848; unneeded family-group name if $\uparrow$ Proscinetes Gistel 1848 is also included in the clade]

Family $\uparrow$ Hadrodontidae Thurmond \& Jones 1981

$\uparrow$ Hadrodontidae Thurmond \& Jones 1981: 82 (family) †Hadrodus Leidy 1857

Family $\uparrow$ Gladiopycnodontidae Taverne \& Capasso 2013

$\dagger$ Gladiopycnodontidae Taverne \& Capasso 2013: 4 (family) †Gladiopycnodus Taverne \& Capasso 2013

Family $\uparrow$ Gebrayelichthyidae Nursall \& Capasso 2004

$\uparrow$ Gebrayelichthyidae Nursall \& Capasso 2004: 318 (family) †Gebrayelichthys Nursall \& Capasso 2004 


\section{Order $\uparrow$ Pholidopleuriformes}

Family $\uparrow$ Pholidopleuridae Abel 1919

$†$ Pholidopleuridae Abel 1919: x (family) $†$ Pholidopleurus Bronn 1858 [also as a new family in Wade 1932: 473]

$\uparrow$ Australosomidae Burton 1931: 45 (family) $\uparrow$ Australosomus Piveteau 1930

Family $\uparrow$ Brachydegmidae Gardiner 1967

$\uparrow$ Brachydegmidae Gardiner 1967: 177 (family) †Brachydegma Dunkle 1939 [family name sometimes seen as $\uparrow$ Brachydegmatidae or $\uparrow$ Brachidegmidae]

\section{Order $\uparrow$ Perleidiformes}

Family $\uparrow$ Platysiagidae Brough 1939

$\uparrow$ Platysiagidae Brough 1939: 14 (family) †Platysiagum Egerton 1872

Family $\uparrow$ Fuyuanperleididae Sun, Lombardo, Tintori, Jiang, Hao, Sun \& Lin 2012

$\uparrow$ Fuyuanperleididae Sun, Lombardo, Tintori, Jiang, Hao, Sun \& Lin 2012: 362 (family) $\uparrow$ Fuyuanperleidus Geng, Jin, Wu \& Wang 2012

Family $\uparrow$ Colobodontidae Andersson [= Stensiö] 1916

$\uparrow$ Colobodontidae Andersson 1916: 17 (family) $\uparrow$ Colobodus Agassiz 1844

$\uparrow$ Asterodontidae Jordan 1923: 112 (family) †Asterodon Münster 1841 [not Asterodon Perrier 1891 in Asteroidea: Odontasteridae]

Family $\uparrow$ Cleithrolepididae Wade 1935, spelling in prevailing recent practice

$\uparrow$ Cleithrolepidae Wade 1935: 47 (family) †Cleithrolepis Egerton 1864 [family name also seen as $\dagger$ Cleithrolepididae]

Family $\dagger$ Gabanelliidae Tintori \& Lombardo 1996

$\uparrow$ Gabanellidae Tintori \& Lombardo 1996: 229 (family) †Gabanellia Tintori \& Lombardo 1996 [correct stem is Gabanelli-]

Family $\uparrow$ Perleididae Brough 1931

$†$ Perleididae Brough 1931: 280 (family) $†$ Perleidus Deecke 1911

Family $\uparrow$ Hydropessidae Hutchinson 1973

$\uparrow$ Hydropessidae Hutchinson 1973: 316 (family) $\uparrow$ Hydropessum Broom 1909

Family $\uparrow$ Pseudobeaconiidae López-Arbarello \& Zavattieri 2008

$†$ Pseudobeaconiidae López-Arbarello \& Zavattieri 2008: 1031 (family) $†$ Pseudobeaconia Bortdas 1944

Family $†$ Eosemionotidae Bürgin, Eichenberger, Furrer \& Tschanz 1991

$†$ Eosemionotidae Bürgin, Eichenberger, Furrer \& Tschanz 1991: 953 (family) †Eosemionotus Stolley 1920

Family $\uparrow$ Cephaloxenidae Brough 1939

†Cephaloxenidae Brough 1939: 19 (family) †Cephaloxenus Brough 1939

\section{Order $\dagger$ Peltopleuriformes}

Family $†$ Polzbergiidae Griffith 1977

$\uparrow$ Polzbergiidae Griffith 1977: 11 (family) †Polzbergia Griffith 1977 [family name sometimes seen as $\uparrow$ Polzbergidae]

Family $\uparrow$ Peltopleuridae Brough 1939

$\uparrow$ Peltopleuridae Brough 1939: 23 (family) $\uparrow$ Peltopleurus Kner 1866

Family $\uparrow$ Habroichthyidae Gardiner 1967

$\dagger$ Habroichthyidae Gardiner 1967: 178 (family) †Habroichthys Brough 1939

Family $\uparrow$ Thoracopteridae Griffith 1977

$\uparrow$ Thoracopteridae Griffith 1977: 1 (family) †Thoracopterus Bronn 1858

Family †Venusichthyidae Xu \& Zhao 2016

†Venusichthyidae Xu \& Zhao 2016: 339 (family) †Venusichthys Xu \& Zhao 2016 


\section{Order $\uparrow$ Luganoiiformes}

Family $\dagger$ Luganoiidae Brough 1939

$\dagger$ Luganoiidae Brough 1939: 39 (family) $\uparrow$ Luganoia Brough 1939 [family name sometimes seen as $\dagger$ Luganoidae]

Family $\uparrow$ Aetheodontidae Brough 1939

$\uparrow$ Aetheodontidae Brough 1939: 51 (family) $\uparrow$ Aetheodontus Brough 1939 [also seen as $\uparrow$ Aethodontus and $\uparrow$ Aethodontidae or $\uparrow$ Aethedontidae]

\section{Division Ginglymodi}

Incertae sedis:

Family $\uparrow$ Kyphosichthyidae Sun \& Ni 2017

$†$ Kyphosichthyidae Sun \& Ni 2017: 67 (family) †Kyphosichthys Xu \& Wu 2012

\section{Order $\uparrow$ Dapediiformes}

Family $\uparrow$ Dapediidae Bleeker 1859

†Dapediden Vogt 1851: 133 (family) †Dapedium Leach 1822 [published not in latinized form before 1900, not available, Art. 11.7.2]

$\dagger$ Dapedioidei Bleeker 1859: XVII (family) $\dagger$ Dapedium Leach 1822 [type genus as $\dagger$ Dapedius Agassiz 1833]

\section{Order Lepisosteiformes}

Family †Obaichthyidae Grande 2010

†Obaichthyidae Grande 2010: 792 (family) †Obaichthys Wenz \& Brito 1992

Family Lepisosteidae Agassiz 1832 [correction of Van der Laan et al. 2014]

Lepidostei Agassiz 1832: 144 (family) Lepisosteus [type genus as Lepidosteus, name must be corrected Art. 32.5.3, see Van der Laan et al. 2014: 28; Agassiz 1833: 10 placed Lepidosteus in Sauroides]

$\uparrow$ Masillosteinae Grande 2010: 661 (subfamily) †Masillosteus Micklich \& Klappert 2001

$\uparrow$ Cuneatini Grande 2010: 792 (tribe) $†$ Cuneatus Grande 2010

\section{Order $†$ Semionotiformes}

Incertae sedis:

Family $\uparrow$ Uarbryichthyidae Bartram 1977

†Uarbryichthyidae Bartram 1977: 204 (family) †Uarbryichthys Wade 1941

Family $†$ Semionotidae Woodward 1890 , name in prevailing recent practice

†Lépidoïdes Agassiz 1833 Tome II pt. 1: 1 (family) †Lepidotes Agassiz 1832 [latinized to $\dagger$ Lepidoidei by Owen 1846: 50, latinized to †Lepidotini by Giebel 1847: 185, latinized to $\uparrow$ Lepidoidea by M'Coy 1855: 605, latinized to †Lepidotidae by Owen 1860: 143; considered valid with this authorship by Bronn 1849: 657, by Eichwald 1868: 1208 and by Cope 1877a: 57 Art. 11.7.2]

$\uparrow$ Stylodontes Wagner 1863: 613 (family) ? [no stem of the type genus, not available, Art. 11.7.1.1] $\uparrow$ Pleurolepididae Lütken 1871: 333 (family) †Pleurolepis Quenstedt 1852 [changed to $\uparrow$ Pleurolepidae by Günther 1880: 366; not the ordinal series name $\uparrow$ Pleurolepidae Quenstedt 1852 / †Lepidopleuridae Young 1866; to avoid confusion it is probably better not to treat $\dagger$ Pleurolepididae in the family-group series]

$\uparrow$ Stylodontidae Günther 1880: 368 (family) ? †Tetragonolepis Bronn 1830 [no stem of the type genus, not available, Art. 11.7.1.1; also preoccupied by $†$ Stylodontidae Marsh 1879 in Mammalia] 
$\dagger$ Sphaerodontidae Günther 1880: 368 (family) †Lepidotes Agassiz 1832 [type genus as $\uparrow$ Lepidotus; no stem of the type genus, not available, Art. 11.7.1.1; also preoccupied by $\dagger$ Sphaerodontidae Giebel 1846 in fishes]

$\uparrow$ Semionotidae Woodward 1890b: 30 (family) $\uparrow$ Semionotus Agassiz 1832

$\uparrow$ Acentrophoridae Berg 1936: 345 (family) $\uparrow$ Acentrophorus Traquair 1877

Family $\dagger$ Callipurbeckiidae López-Arbarello 2012

$\uparrow$ Paralepidotidae Stolley 1920: 53 (family) $\uparrow$ Paralepidotus Stolley 1920 [mentioned as a possible new family name, not proposed conditionally in the sense of Art. 15; not available]

$†$ Callipurbeckiidae López-Arbarello 2012: 35, 41 (family) †Callipurbeckia López-Arbarello 2012 [family name sometimes seen as $\uparrow$ Callipurbeckidae]

Family $\uparrow$ Macrosemiidae Wagner 1860 , name in prevailing recent practice

"une troisième famille qui réduira un peu le nombre de ces formes génériques encore flottantes. Elle aura pour type le genre $\uparrow$ Macrosemius, A." Thiollière 1858: 791 (family) $\dagger$ Macrosemius Agassiz 1834 [no family name used, not available]

$\uparrow$ Propteroidei Bleeker 1859: XVII (family) †Propterus Agassiz 1834

$\dagger$ Macrosemii Wagner 1860a: 399 (Gruppe) †Macrosemius Agassiz 1834 [corrected to $\uparrow$ Macrosemiidae by Cope 1889: 858]

\section{Division Halecomorphi}

Incertae sedis:

$\dagger$ Gigantodontidae Weiler 1935: 26, 29 (family) †Stromerichthys Weiler 1935 [no stem of the type genus, not available, Art. 11.7.1.1]

$†$ Pingolepidae Chang \& Chou 1974: 184 (family) †Pingolepis Chang \& Chou 1974 [also Chang \& Chou 1977: 23, 56]

†Praesemionotidae Jörg 1969: 89, 94 (family) †Praesemionotus Jörg 1969

\section{Order $\uparrow$ Parasemionotiformes}

Family $\uparrow$ Parasemionotidae Stensiö 1931

†Ospiidae Stensiö in Koch 1931: 74 (family) †Ospia Stensiö 1932 [no valid type genus, not available, Art. 11.7.1.1]

$\uparrow$ Bronghiidae [†Broughiidae] Koch 1931: 88 (family) ? †Broughia Stensiö 1932 [no valid type genus, not available, Art. 11.7.1.1]

$†$ Parasemionotidae Stensiö in Koch 1931: 74 (family) †Parasemionotus Piveteau 1929 [type genus inferred from the stem; name only, but used as valid by Stensiö 1932a: 97, 183, by Gardiner 1960: 333, by Griffith \& Patterson 1963: 33, by Romer 1966: 354, by Andrews et al. 1967: 652, by Fowler 1971c: 403 and by Gardiner 1993b: 618 Art. 13.2.1]

†Ospiidae Stensiö 1932a: 97, 183 (family) †Ospia Stensiö 1932

$\uparrow$ Tungusichthyidae Berg 1940: 406 (family) $†$ Tungusichthys Berg 1941 [no valid type genus, not available, Art. 11.7.1.1]

$\dagger$ Tungusichthyidae Berg 1941: 462 (family) †Tungusichthys Berg 1941

$†$ Promecosominidae Wade 1941a: 380 (family) †Promecosomina Wade 1935

$\dagger$ Paracentrophoridae Gardiner 1960: 347 (family) †Paracentrophorus Piveteau 1941

\section{Order $\dagger$ Panxianichthyiformes}

Family $\uparrow$ Panxianichthyidae Sun, Tintori, Xu, Lombardo, Ni \& Jiang 2016

$\uparrow$ Panxianichthyidae Sun, Tintori, Xu, Lombardo, Ni \& Jiang 2016: 224 (family) $†$ Panxianichthys Xu \& Shen 2015

\section{Order $\dagger$ Ophiopsiformes}

Incertae sedis:

$\dagger$ Furidae Jordan 1923: 116 (family) $\uparrow$ Furo Gistel 1848 [family name sometimes seen as $†$ Furonidae] 
†Ophiopsidae Bartram 1975: 201 (family) †Ophiopsis Agassiz 1834 [family name sometimes seen as $†$ Ophiopsididae]

Family $†$ Ophiopsiellidae Ebert 2018

†Ophiopsiellidae Ebert 2018: [15] (family) †Ophiopsiella Lane \& Ebert 2015

\section{Order Amiiformes}

Family †Ionoscopidae Lehman 1966

$\dagger$ Ionoscopidae Lehman 1966: 141 (family) †Ionoscopus Costa 1853

†Oshuniidae Grande \& Bemis 1998: 606 (family) †Oshunia Wenz \& Kellner 1986

\section{Suborder $\uparrow$ Caturoidei}

Family $\uparrow$ Caturidae Owen 1860 , name in prevailing recent practice

$\dagger$ Trapeziolepidoidei Bleeker 1859: XVI (family) †Caturus Agassiz 1834 [no stem of the type genus, not available, Art. 11.7.1.1]

†Lophiostomatoidei Bleeker 1859: XVII (family) †Lophiostomus Egerton 1852

$\uparrow$ Caturidae Owen 1860: 139 (family) $†$ Caturus Agassiz 1834 [also $†$ Caturini Wagner 1860a: 396, published later]

$\uparrow$ Cyclolepidoti Zittel 1887: 227 (family) ? †Caturus Agassiz 1834 [no stem of the type genus, not available, Art. 11.7.1.1]

$\dagger$ Eugnathidae Lydekker in Nicholson \& Lydekker 1889: 986 (family) †Eugnathus Agassiz 1844 [type genus preoccupied by Eugnathus Schönherr 1833 in Coleoptera; invalid, Art. 39]

$\dagger$ Isopholidae Hay 1899: 790 (family) †Isopholis Zittel 1887

$\dagger$ Liodesmidae Jordan 1905: 34 (family) †Liodesmus Wagner 1859

\section{Suborder Amioidei}

Family $\uparrow$ Sinamiidae Berg 1940

$\dagger$ Sinamiidae Berg 1940: 412 (family) $†$ Sinamia Stensiö 1935

Family Amiidae Bonaparte 1831

$†$ Megaluridae Zittel 1895: 584 (family) $†$ Megalurus Agassiz 1833 [type genus preoccupied by Megalurus Horsfield 1821 in Aves; invalid, Art. 39; not Megaluridae in Aves]

†Vidalamiinae Grande \& Bemis 1998: 337 (subfamily) †Vidalamia White \& Moy-Thomas 1941 [also as new tribe $\uparrow$ Vidalamiini]

†Solnhofenamiinae Grande \& Bemis 1998: 450 (subfamily) †Solnhofenamia Grande \& Bemis 1998 $\uparrow$ Amiopsinae Grande \& Bemis 1998: 483 (subfamily) †Amiopsis Kner 1863

$\uparrow$ Calamopleurini Grande \& Bemis 1998: 405 (tribe) Calamopleurus Agassiz 1841

\section{Superdivision TELEOSTEOMORPHA}

Incertae sedis:

$\uparrow$ Palaeolabridae Estes 1969b: 2 (family) †Palaeolabrus Casier 1967

$\uparrow$ Pseudoberycidae Berg 1940: 420 (family) $\uparrow$ Pseudoberyx Pictet \& Humbert 1866

\section{Division $\uparrow$ Aspidorhynchei}

Order $\uparrow$ Aspidorhynchiformes

Family $\uparrow$ Aspidorhynchidae Bleeker 1859

$\uparrow$ Aspidorhynchoidei Bleeker 1859: XVI (family) †Aspidorhynchus Agassiz 1833

$\uparrow$ Vinctiferidae Silva Santos 1990: 252 (family) †Vinctifer Jordan 1920

\section{Order $\uparrow$ Pachycormiformes}

Family $\dagger$ Pachycormidae Lydekker 1889 , name in prevailing recent practice

$\dagger$ Saurotomini [ $†$ Saurostomini] Bonaparte 1846: 4 (subfamily) †Saurostomus Agassiz 1843 [genus inferable from the stem?; corrected to †Saurostomini by Bonaparte 1850a: 455; corrected to $†$ Saurostomata by Fitzinger 1873: 50] 
$\dagger$ Pelecopteridae Cope 1875: 244A (family) †Pelecopterus Cope 1875

$\dagger$ Sauropsidae Cope 1877a: 60 (family) †Sauropsis Agassiz 1832

$\dagger$ Erisichtheidae Cope 1877b: 822 (family) $†$ Erisichthe Cope 1872 [family name sometimes seen as $\dagger$ Erisichthidae or $\dagger$ Eriscichtheidae]

$\uparrow$ Microlepidoti Zittel 1887: 223 (family) ? †Pachycormus Agassiz 1833 [no stem of the type genus, not available, Art. 11.7.1.1]

$\uparrow$ Pachycormidae Lydekker in Nicholson \& Lydekker 1889: 989 (family) †Pachycormus Agassiz 1833

†Protosphyraenidae Lydekker in Nicholson \& Lydekker 1889: 996 (family) †Protosphyraena Leidy 1857

$†$ Diphyodontidae Jordan 1923: 113 (family) †Diphyodus Lambe 1902

\section{Order $\uparrow$ Prohaleciteiformes}

Family $\uparrow$ Prohaleciteidae Arratia 2017

$\uparrow$ Prohaleciteidae Arratia 2017: 23 (family) †Prohalecites Deeke 1889

\section{Division Teleostei}

Incertae sedis:

Family $\uparrow$ Sorbinicharacidae Taverne 2003

†Sorbinicharacidae Taverne 2003: 29 (family) †Sorbinicharax Taverne 2003

\section{Order $\uparrow$ Pholidophoriformes}

$\dagger$ Mjollnirulidae Lund 1968 (family) †'Mjollnirulus' [unavailable publication, family-group name not available]

$\dagger$ Nybelineidae Lund 1968 (family) †'Nybelinea' [not Nybelinea Whitley 1976: 48; unavailable publication, family-group name not available]

$†$ †jalvidae Lund 1968 (family) †'Tjalvis' [unavailable publication, family-group name not available]

Family $\dagger$ Ligulellidae Saint-Seine 1955

$\dagger$ Ligulellidae Saint-Seine 1955: 103 (family) †Ligulella Saint-Seine 1955

Family $†$ Siyuichthyidae Su 1980

$\dagger$ Siyuichthyidae Su 1980: 77 (family) †Siyuichthys Su 1980 [also Su 1985: 73, 130]

Family $\dagger$ Eurycormidae Arratia 2017

$\dagger$ Eurycormidae Arratia 2017: 23 (family) †Eurycormus Wagner 1863

Family $\uparrow$ Pholidophoridae Wagner 1860

$\dagger$ Monostichii Giebel 1847: 203 (family) †Pholidophorus Agassiz 1832 [no stem of the type genus, not available, Art. 11.7.1.1]

$\dagger$ Pholidophori Wagner 1860b: 402 (Gruppe) †Pholidophorus Agassiz 1832

Family $\uparrow$ Archaeomaenidae Boulenger 1904

$\uparrow$ Archaeomaenidae Boulenger 1904a: 163 (family) †Archaeomaene Woodward 1895

$\uparrow$ Aetheolepidae Wade 1941b: 80 (family) †Aetheolepis Woodward 1895

$\uparrow$ Aphnelepidae Wade 1941b: 78 (family) $\uparrow$ Aphnelepis Woodward 1895 [family name sometimes seen as $\uparrow$ Aphnelepididae]

Family $\uparrow$ Protelopidae Saint-Seine 1949

$\uparrow$ Protelopidae Saint-Seine 1949: 261 (family) $†$ Protelops Laube 1885

Family $\uparrow$ Pleuropholidae Saint-Seine 1949

$†$ Pleuropholidae Saint-Seine 1949: 249, 255 (family) †Pleuropholis Egerton 1858 [family name sometimes seen as $\uparrow$ Pleuropholididae]

Family $\uparrow$ Galkiniidae Yakovlev 1962

$\dagger$ Galkiniidae Yakovlev 1962: 93 (family) †Galkinia Berg 1949 
Family $\uparrow$ Majokiidae Saint-Seine 1955

$\uparrow$ Majokiidae Saint-Seine 1955: 107 (family) †Majokia Saint-Seine 1955

Family $\uparrow$ Oligopleuridae Woodward 1895

†Oligopleuridae Woodward 1895: 490 (family) †Oligopleurus Thiollière 1850

Family $\uparrow$ Catervariolidae Saint-Seine 1955

$\uparrow$ Catervariolidae Saint-Seine 1955: 18 (family) †Catervariolus Saint-Seine 1955

Family $\uparrow$ Ichthyokentemidae Griffith \& Patterson 1963

$\dagger$ Ichthyokentemidae Griffith \& Patterson 1963: 6 (family) †Ichthyokentema Woodward 1941

[family name sometimes seen as $\uparrow$ Ichthyokentematidae]

Family $\uparrow$ Ankylophoridae Gaudant 1978

$\uparrow$ Ankylophoridae Gaudant 1967b: 373 (family) †Ankylophorus Gaudant 1978 [no valid type genus, not available, Art. 11.7.1.1]

$\uparrow$ Ankylophoridae Gaudant 1978: 113 (family) $\uparrow$ Ankylophorus Gaudant 1978

\section{Order $\dagger$ Dorsetichthyiformes}

Family $\uparrow$ Dorsetichthyidae

†Dorsetichthyidae Nelson, Grande \& Wilson 2016: 130 (family) †Dorsetichthys Arratia 2013 [not published according to the rules, not available]

$\uparrow$ Dorsetichthyidae Arratia 2017: 24 (family) †Dorsetichthys Arratia 2013 [not published according to the rules, not available]

\section{Order $\dagger$ Leptolepidiformes}

Family $\uparrow$ Leptolepididae Owen 1860, spelling in prevailing recent practice

$†$ Leptolépides Pictet 1854: 135 (family) †Leptolepis Agassiz 1832 [published not in latinized form before 1900, not available, Art. 11.7.2]

$\uparrow$ Cyclolepidoidei Bleeker 1859: XVI (family) †Leptolepis Agassiz 1832 [no stem of the type genus, not available, Art. 11.7.1.1]

$\dagger$ Leptolepidae Owen 1860: 144 (family) †Leptolepis Agassiz 1832 [family name also seen as $\dagger$ Leptolepididae]

$? \uparrow$ Ctenolepides Fitzinger 1873: 50 (family) $†$ Ctenolepis Agassiz 1844 [family uncertain]

Family $\uparrow$ Koonwarriidae Waldman 1971

$†$ Koonwarriidae Waldman 1971: 35 (family) †Koonwarria Waldman 1971

Family $\uparrow$ Ascalaboidae Arratia 2016

$\uparrow$ Ascalaboidae Arratia 2016: 33 (family) †Ascalabos Münster 1839 [family name sometimes seen as $\uparrow$ Ascalabidae]

\section{Order $\uparrow$ Crossognathiformes}

Incertae sedis:

$†$ Salminopsidae Gayet 1985b: 896 (family) †Salminops Gayet 1985 [also in Gayet 1985c: 93]

Family $\uparrow$ Varasichthyidae Arratia 1981

†Varasichthyidae Arratia 1981: 110 (family) †Varasichthys Arratia 1981

Family $\uparrow$ Chongichthyidae Arratia 1982

$\dagger$ Chongichthyidae Arratia 1982: 132 (family) †Chongichthys Arratia 1982

Family $\uparrow$ Crossognathidae Woodward 1901, name in prevailing recent practice

$†$ Pelecorapidae Cragin 1901: 30 (family) †Pelecorapis Cope 1875 [Hay 1929: 734 selected $\dagger$ Pelecorapidae over $\uparrow$ Syllaemidae, confirmed by Fowler 1973: 296; family name sometimes seen as $\dagger$ Pelycorapidae]

$\dagger$ Syllaemidae Cragin 1901: 25 (family) $†$ Syllaemus Cope 1875

$†$ Crossognathidae Woodward 1901: 348 (family) †Crossognathus Pictet 1858 
$\uparrow$ Apsopelicidae Romer 1966: 354 (family) †Apsopelix Cope 1871 [name only, published after 1960, not available, Art. 13.1.1]

Family $\uparrow$ Notelopidae Forey 1977

$\uparrow$ Notelopidae Forey 1977: 129 (family) †Notelops Woodward 1901

Family $\uparrow$ Pachyrhizodontidae Cope 1872

$\uparrow$ Pachyrhizodontidae Cope 1872: 343 (family) †Pachyrhizodus Dixon 1850 [family name sometimes seen as †Pachyrhizodidae or $\uparrow$ Pachyrhizolidae]

$\uparrow$ Thrissopatrinae Boulenger 1904b: 562 (subfamily) †Thrissopater Günther 1872 [family name sometimes seen as $\uparrow$ Thrissopateridae]

$\dagger$ Raphiosauridae Fowler 1911: 151 (family) $†$ Raphiosaurus Owen 1842

$\dagger$ Greenwoodellidae Taverne 1973: 5 (family) †Greenwoodella Taverne \& Ross 1973

\section{Order $\dagger$ Ichthyodectiformes}

Family $\uparrow$ Allothrissopidae Patterson \& Rosen 1977, name in prevailing recent practice

$\uparrow$ Thressopina [†Thrissopina] Bassani 1879a: 167 (Gruppe) †Thrissops Agassiz 1833 [corrected to $†$ Thrissopina by Bassani 1879b: 204]

$\uparrow$ Allothrissopidae Patterson \& Rosen 1977: 115, 163 (family) $\uparrow$ Allothrissops Nybelin 1964

†Occithrissopidae Taverne \& Chanet 2000: 32 (family) †Occithrissops Schaeffer \& Patterson 1984 [not published according to the rules, not available]

$\dagger$ Unamichthyidae Alvarado-Ortega 2004: 803 (family) †Unamichthys Alvarado-Ortega 2004

Family $\uparrow$ Chuhsiungichthyidae Yabumoto 1994

$\uparrow$ Chuhsiungichthiidae [ $†$ Chuhsiungichthyidae] Yabumoto 1994: 130 (family) $\uparrow$ Chuhsiungichthys Lew 1974

Family $\uparrow$ Cladocyclidae Maisey 1991

$\uparrow$ Cladocyclidae Maisey 1991: 190, 206 (family) †Cladocyclus Agassiz 1841

Family $\uparrow$ Saurodontidae Bonaparte 1846

$\dagger$ Saurodontidae Bonaparte 1846: 4 (family) †Saurodon Hays 1830 [type genus inferred from the stem, Art. 11.7.1.1; also as subfamily $\dagger$ Saurodontini]

$\uparrow$ Saurocephalidae Zittel 1888: 262 (family) †Saurocephalus Harlan 1824

$\dagger$ Gillicinae Taverne 2008: 224 (subfamily) †Gillicus Hay 1898

Family †Ichthyodectidae Crook 1892

$\dagger$ Ichthyodectidae Crook 1892: 111, 121 (family) †Ichthyodectes Cope 1870 [family name sometimes seen as $\uparrow$ Icthyodectidae based on $\uparrow$ Icthyodectes]

Family †Luisiellidae Sferco, López-Arbarello \& Báez 2015

†Luisiellidae Sferco, López-Arbarello \& Báez 2015: 13 (family) †Luisiella Bocchino 1967

\section{Order $\uparrow$ Tselfatiiformes}

Family $\uparrow$ Protobramidae Le Danois \& Le Danois 1964

$\uparrow$ Protobramidae Le Danois \& Le Danois 1964: 185 (family) †Protobrama Woodward 1942

Family $\uparrow$ Eoplethodidae Taverne \& Gayet 2005

$\dagger$ Eoplethodidae Taverne \& Gayet 2005: 66 (family) †Eoplethodus Taverne 2000 [family name sometimes seen as $\dagger$ Eoplethodontidae]

Family $\uparrow$ Plethodidae Loomis 1900 [ICZN Opinion 921]

$\uparrow$ Plethodidae Loomis 1900: 229 (family) †Plethodus Dixon 1850 [changed to †Plethodontidae by Hay 1929: 736, but Plethodontidae preoccupied in Amphibia; the incorrect subsequent spelling †Plethodontidae is invalid, ICZN Opinion 921]

$\uparrow$ Thryptodontidae Jordan 1905: 44 (family) †Thryptodus Loomis 1900 [family name sometimes seen as $\uparrow$ Thryptodidae]

$\uparrow$ Anogmiidae Jordan 1925a: 219, 220 (family) †Anogmius Cope 1877 [type genus preoccupied by $\uparrow$ Anogmius Cope 1871 in fishes; invalid, Art. 39] 
$\uparrow$ Niobraridae Jordan 1925a: 222 (family) $\uparrow$ Niobrara Jordan 1925

$\dagger$ Tselfatidae Arambourg 1944: 282 (family) $\uparrow$ Tselfatia Arambourg 1944 [family name also seen as $\dagger$ Tselfatiidae; correct stem is Tselfati-]

$\dagger$ Bananogmiidae Applegate 1970: 413 (family) †Bananogmius Whitley 1940 [family name sometimes seen as $\uparrow$ Ananogmiidae, possibly based on $\uparrow$ Ananogmius White \& Moy Thomas 1940]

\section{Order $\uparrow$ Araripichthyiformes}

Family $\uparrow$ Araripichthyidae Silva Santos 1985

$\uparrow$ Araripichthyidae Silva Santos 1985: 135 (family) $†$ Araripichthys Silva Santos 1985

\section{Cohort ELOPOMORPHA}

Family †Anaethalionidae Gaudant 1967

$\uparrow$ Anaethalionidae Gaudant 1967: 309 (family) †Anaethalion White 1938 [extended description in Gaudant 1968; family name sometimes seen as $\uparrow$ Anaethaliidae]

\section{Order Elopiformes}

Family Elopidae Valenciennes 1847

Family Megalopidae Jordan \& Gilbert 1883

$\dagger$ Sedenhorstiidae Goody 1969a: 3 (family) †Sedenhorstia White \& Moy-Thomas 1941

Family $\uparrow$ Phyllodontidae Sauvage 1875, spelling in prevailing recent practice

$\uparrow$ Phyllodidae Sauvage 1875: 615 (family) $\uparrow$ Phyllodus Agassiz 1839 [family name also seen as $\uparrow$ Phyllodontidae]

†Euphyllodontinae Dartevelle \& Casier 1949: 229 (subfamily) ? [no valid type genus, not available, Art. 11.7.1.1]

$†$ Pseudophyllodontinae Dartevelle \& Casier 1949: 229 (subfamily) ? [no valid type genus, not available, Art. 11.7.1.1]

$\uparrow$ Paralbulinae Estes 1969a: 321 (subfamily) $\uparrow$ Paralbula Blake 1940

\section{Order Albuliformes}

Family †Osmeroididae Forey 1973

†Osmeroididae Forey 1973: 94 (family) †Osmeroides Agassiz 1837

Family $\uparrow$ Eurokidae Bartholomai 2010

$\uparrow$ Eurokidae Bartholomai 2010: 71 (family) †Euroka Bartholomai 2010

Family Albulidae Bleeker 1849

\section{Order Notacanthiformes}

Family Halosauridae Günther 1868

Family Notacanthidae Rafinesque 1810

$\dagger$ Pronotacanthidae Jordan 1923: 128 (family) †Pronotacanthus Woodward 1900

\section{Order Anguilliformes}

Incertae sedis:

$\uparrow$ Derrhiidae Jordan 1925b: 15 (family) $\uparrow$ Derrhias Jordan 1925

Family $\uparrow$ Anguillavidae Hay 1903

$\uparrow$ Anguillavidae Hay 1903: 436 (family) $\uparrow$ Anguillavus Hay 1903

$\uparrow$ Encheliidae Hay 1903: 441 (family) $\uparrow$ Enchelion Hay 1903

$\uparrow$ Mylomyridae Berg 1940: 450 (family) $†$ Mylomyrus Woodward 1910

Family $\uparrow$ Georgidentidae Sytchevskaya \& Prokofiev 2004

$\uparrow$ Georgidentidae Sytchevskaya \& Prokofiev 2004: 23 (family) †Georgidens Sytchevskaya \& Prokofiev 2004 
Family $\dagger$ Libanechelyidae Taverne 2004

$\dagger$ Libanechelyidae Taverne 2004: 74 (family) †Libanechelys Taverne 2004

Family $\uparrow$ Milananguillidae Blot 1978

$\dagger$ Milananguillidae Blot 1976a: 510 (family) $†$ Milananguilla Blot 1978 [no valid type genus, not available, Art. 11.7.1.1]

$\dagger$ Milananguillidae Blot 1978: 75 (family) $†$ Milananguilla Blot 1978

Family †Urenchelyidae Jordan 1905

$†$ Urenchelyidae Jordan 1905: 142 (family) †Urenchelys Woodward 1900 [family name sometimes seen as $\dagger$ Urenchelidae]

\section{Suborder Protanguilloidei}

Family Protanguillidae Johnson, Ida \& Miya 2012

Suborder Synaphobranchoidei

Family Synaphobranchidae Johnson 1862

Suborder Moringuoidei

Family Moringuidae Gill 1885

Suborder Anguilloidei

Family $\uparrow$ Anguilloididae Blot 1978

$\uparrow$ Anguilloididae Blot 1976: 510 (family) $\uparrow$ Anguilloides Cadrobbi 1962 [name only, published after 1960, not available, Art. 13.1.1]

$\uparrow$ Anguilloididae Blot 1978: 100 (family) $\uparrow$ Anguilloides Cadrobbi 1962

Family $\uparrow$ Patavichthyidae Blot 1981

$\dagger$ Patavichthyidae Blot 1976: 510 (family) †Patavichthys Blot 1981 [no valid type genus, not available, Art. 11.7.1.1]

$\uparrow$ Patavichthidae Blot 1981: 349 (family) †Patavichthys Blot 1981 [family name also seen as $\uparrow$ Patavichthyidae; correct stem is Patavichthy-]

Family $\uparrow$ Proteomyridae Blot 1981

$\dagger$ Proteomyridae Blot 1976: 510 (family) †Proteomyrus Cadrobbi 1962 [name only, published after 1960, not available, Art. 13.1.1]

$\dagger$ Proteomyridae Blot 1981: 348 (family) †Proteomyrus Cadrobbi 1962

Family Nemichthyidae Kaup 1859

Family Anguillidae Rafinesque 1810

Suborder Muraenoidei

Family $\uparrow$ Paranguillidae Blot 1981

$\dagger$ Paranguillidae Blot 1976: 510 (family) $\uparrow$ Paranguilla Bleeker 1864 [name only, published after 1960, not available, Art. 13.1.1]

$\uparrow$ Paranguillidae Blot 1981: 346 (family) $†$ Paranguilla Bleeker 1864

Family Serrivomeridae Trewavas 1932

Family Heterenchelyidae Regan 1912

Family Myrocongridae Gill 1890

Family Muraenidae Rafinesque 1815

Suborder Chlopsoidei

Family Chlopsidae Rafinesque 1815

Suborder Congroidei

Family Congridae Kaup 1856

$\dagger$ Bolcyridae White in Blot 1984: 234 (family) $†$ Bolcyrus Blot 1978

Family Derichthyidae Gill 1884

Family Nettastomatidae Kaup 1859

Family Muraenesocidae Kaup 1859

Family $\uparrow$ Nardoechelyidae Taverne \& Capasso 2014

$\uparrow$ Nardoechelyidae Taverne \& Capasso 2014: 4 (family) $\uparrow$ Nardoechelys Taverne 2002 
Family Ophichthidae Günther 1870

$\uparrow$ Parechelidae Casier 1967: 8 (family) $†$ Parechelus Casier 1967

$\uparrow$ Asanoinae Sytchevskaya \& Prokofiev 2004: 29 (subfamily) $\uparrow$ Asanoa Sytchevskaya \& Prokofiev 2004

Suborder Saccopharyngoidei

Family Cyematidae Regan 1912

Family Monognathidae Trewavas 1937

Family Saccopharyngidae Bleeker 1859

Family Eurypharyngidae Gill 1883

\section{Cohort OSTEOGLOSSOMORPHA}

Incertae sedis:

Family $\dagger$ Jiuquanichthyidae Ma 1993

$\dagger$ Jiuquanichthyidae Ma 1993: 23, 92 (family) †Jiuquanichthys Ma 1993

Family $\uparrow$ Kipalaichthyidae Taverne 1976

$\dagger$ Kipalaichthyidae Taverne 1976: 25, 30 (family) $†$ Kipalaichthys Taverne 1976

Family $\uparrow$ Kuyangichthyidae Liu, Ma \& Liu 1982

$\dagger$ Kuyangichthyidae Liu, Ma \& Liu 1982: 101 (family) †Kuyangichthys Liu, Ma \& Liu 1982

\section{Order $\uparrow$ Lycopteriformes}

Family †Lycopteridae Cockerell 1924

$†$ Lycopteridae Cockerell 1924: 131 (family) †Lycoptera Müller 1848

$\uparrow$ Manchurichthynae [ $†$ Manchurichthyinae] Takai 1944: 248 (subfamily) $†$ Manchurichthys Saito 1936 [correct stem is Manchurichthy-]

Family $†$ Wakinichthyidae Yabumoto 1994

$\dagger$ Wakinichthiidae [†Wakinichthyidae] Yabumoto 1994: 179 (family) †Wakinichthys Yabumoto 1994 [correct stem is Wakinichthy-; family name sometimes seen as †Wakinichthidae or †Wakinoichthidae]

\section{Order Hiodontiformes}

Family Hiodontidae Valenciennes 1847

$\dagger$ Jiaohichthyidae Ma 1983: 28 (family) †Jiaohichthys Ma 1983

\section{Order Osteoglossiformes}

Family †Ostariostomidae Schaeffer 1949

†Ostariostomidae Schaeffer 1949: 3 (family) †Ostariostoma Schaeffer 1949

Family $\uparrow$ Huashiidae Chang \& Chou 1974

$\dagger$ Huashiidae Chang \& Chou 1974: 185 (family) †Huashia Chang \& Chou 1974 [also Chang \& Chou 1977: 39, 57]

Family Pantodontidae Peters 1876

$\uparrow$ Singididae Greenwood \& Patterson 1967: 213 (family) †Singida Greenwood \& Patterson 1967

Family Osteoglossidae Bonaparte 1845

$†$ Phareodontidae Jordan 1925a: 232 (family) $†$ Phareodus Leidy 1873 [family name sometimes seen as †Phareodidae]

$†$ Brychaetidae Bonde 1966: 199 (family) †Brychaetus Woodward 1901

$†$ Laeliichthyinae Silva Santos 1985b: 161 (subfamily) †Laeliichthys Silva Santos 1985

$†$ †oreyichthyidae Taverne 1998: 67 (family) Foreyichthys Taverne 1979

Family Notopteridae Bleeker 1851

Family Mormyridae Bonaparte 1831

Family Gymnarchidae Bleeker 1859 


\section{Cohort CLUPEOCEPHALA}

Incertae sedis:

$\nmid$ Lycoclupeidae Gowda 1967: 119, 123 (family) †Lycoclupea Gowda 1967

\section{Subcohort Otomorpha}

Incertae sedis:

Family $\uparrow$ Clupavidae Bertin \& Arambourg 1958

†Clupavidae Bertin \& Arambourg 1958: 2230 (family) †Clupavus Arambourg 1950

Family $\uparrow$ Ganolytidae Jordan 1923

$\dagger$ Ganolytidae Jordan 1923: 118 (family) †Ganolytes Jordan 1919

\section{Superorder Clupei}

Order $\uparrow$ Ellimichthyiformes

Family $†$ Sorbinichthyidae Bannikov \& Bacchia 2000

$†$ Sorbinichthyidae Bannikov \& Bacchia 2000: 4 (family) †Sorbinichthys Bannikov \& Bacchia 2000

Suborder $\uparrow$ Armigatoidei

Family $\uparrow$ Armigatidae Murray \& Wilson 2013

$†$ Diplomystidae Patterson 1970: 179 (family) †Diplomystus Cope 1877 [Diplomystidae preoccupied in fishes; not to be used, Art. 55.3]

$\uparrow$ Armigatidae Murray \& Wilson 2013: 282 (family) $†$ Armigatus Grande 1982

Suborder $\dagger$ Ellimichthyoidei

Family $\uparrow$ Paraclupeidae Chang \& Chou 1974

$\uparrow$ Paraclupeidae Chang \& Chou 1974: 185 (family) $\uparrow$ Paraclupea Du $1950[=? \uparrow$ Paraclupea Sun 1956] [also Chang \& Chou 1977: 28, 56]

$†$ Ellimmichthyidae Grande 1982: 5 (family) †Ellimichthys Jordan 1919 [type genus as $\dagger$ Ellimmichthys, name must be corrected Art. 32.5.3; correct stem is Ellimichthy-]

†Scutatuspinosinae Silva Santos \& Silva Corréa 1985: 169 (subfamily) †Scutatuspinosus Silva Santos \& Silva Corréa 1985

$\dagger$ Ellimminae Murray \& Wilson 2013: 282 (subfamily) †Ellimma Jordan 1913

$\uparrow$ Thorectichthyinae Murray \& Wilson 2013: 282 (subfamily) †Thorectichthys Murray \& Wilson 2013

$\dagger$ Triplomystini Murray \& Wilson 2013: 282 (tribe) $†$ Triplomystus Forey, Lu, Patterson \& Davies 2003

\section{Order Clupeiformes}

\section{Suborder Denticipitoidei}

Family Denticipitidae Clausen 1959

\section{Suborder Clupeoidei}

Family $\uparrow$ Garganoclupeidae Taverne 2014

† Garganoclupeidae Taverne 2014: 28 (family) †Garganoclupea Taverne 2014

Family Pristigasteridae Bleeker 1872

Family Engraulidae Gill 1861

Family Chirocentridae Bleeker 1849

Family Clupeidae Cuvier 1816

\section{Superorder Alepocephali}

Order Alepocephaliformes

Family Alepocephalidae Bonaparte 1846

Family Platytroctidae Koefoed 1927 
Order $\uparrow$ Sorbininardiformes

Family †Sorbininardidae Taverne 1999

†Sorbininardidae Taverne 1999: 77 (family) †Sorbininardus Taverne 1999

\section{Superorder Ostariophysi}

Incertae sedis:

$\uparrow$ Ancylostylidae Jordan 1923: 119 (family) †Ancylostylos Gorjanović-Kramberger 1895

$\dagger$ Erythrinolepidae Cockerell 1919: 182 (family) †Erythrinolepis Cockerell 1919

Section Anotophysa

Order Gonorynchiformes

Family Chanidae Günther 1868

$\uparrow$ Rubiesichthyinae Poyato-Ariza 1996: 5, 35 (subfamily) $\uparrow$ Rubiesichthys Wenz 1984

Family $\uparrow$ Apulichthyidae Taverne 1997

$\uparrow$ Apulichthyidae Taverne 1997: 402 (family) $†$ Apulichthys Taverne 1997

Family Gonorynchidae Fowler 1941 (1848) [correction of Van der Laan et al. 2014]

$\uparrow$ Notogoneidae Cockerell in Jordan 1923: 120 (family) $\uparrow$ Notogoneus Cope 1885

$\dagger$ Judeichthyidae Gayet 1985a: 66 (family) $†$ Judeichthys Gayet 1985

$\uparrow$ Charitosomidae Gayet 1993: 1 (family) †Charitosomus von der Marck 1885

Family Kneriidae Günther 1868

Section Otophysa

Family $†$ Chanoididae Taverne 2005

$\uparrow$ Chanoididae Taverne 2005: 39 (family) †Chanoides Woodward 1901

\section{Order Cypriniformes}

Suborder Cyprinoidei

Family Psilorhynchidae Hora 1926

Family Cyprinidae Rafinesque 1815

Family Danionidae Bleeker 1863

Family Paedocyprididae Mayden \& Chen 2010

Family Sundadanionidae Mayden \& Chen 2010

Family Leptobarbidae Bleeker 1864

Family Xenocyprididae Günther 1868

Family Acheilognathidae Bleeker 1863

Family Tincidae Jordan 1878

Family Gobionidae Bleeker 1863

Family Tanichthyidae Mayden \& Chen 2010

Family Leuciscidae Bonaparte 1835

\section{Suborder Cobitoidei}

Family $\uparrow$ Jianghanichthyidae Liu, Chang, Wilson \& Murray 2015

$\dagger$ Jianghanichthyidae Liu, Chang, Wilson \& Murray 2015: 4 (family) †Jianghanichthys Lei 1987

Family Catostomidae Agassiz 1850

Family Gyrinocheilidae Gill 1905

Family Botiidae Berg 1940

Family Vaillantellidae Nalbant \& Bănărescu 1977

Family Cobitidae Swainson 1838 [ICZN Opinion 1500]

$\dagger$ Acanthopsides Heckel \& Kner 1857: 296 (family) $†$ Acanthopsis Agassiz 1832

Family Balitoridae Swainson 1839

Family Barbuccidae Kottelat 2012 
Family Gastromyzontidae Fowler 1905

Family Serpenticobitidae Kottelat 2012

Family Ellopostomatidae Bohlen \& Šlechtová 2009

Family Nemacheilidae Regan 1911

Order Characiformes

Suborder Citharinoidei

Family Distichodontidae Günther 1864

Family Citharinidae Günther 1864

Suborder Characoidei

Family Crenuchidae Günther 1864

Family Ctenoluciidae Schultz 1944

Family Lebiasinidae Gill 1889

Family Chalceidae Fowler 1958

Family Acestrorhynchidae Eigenmann 1912

Family Iguanodectidae Eigenmann 1909

Family Triportheidae Fowler 1940

Family Gasteropelecidae Bleeker 1859

Family Bryconidae Eigenmann 1912

Family Characidae Latreille 1825

Family Alestidae Cockerell 1910

Family Hepsetidae Hubbs 1939 (1909)

Family Tarumaniidae de Pinna, Zuanon, Rapp Py-Daniel \& Petry, 2017

Family Erythrinidae Valenciennes 1847

Family Cynodontidae Eigenmann 1903

Family Hemiodontidae Bleeker 1859

Family Serrasalmidae Bleeker 1859

Family Parodontidae Eigenmann 1910

Family Prochilodontidae Eigenmann 1909

Family Curimatidae Gill 1858

Family Chilodontidae Eigenmann 1903

Family Anostomidae Günther 1864

Order Gymnotiformes

Suborder Gymnotoidei

Family Gymnotidae Rafinesque 1815

Suborder Sternopygoidei

Family Rhamphichthyidae Regan 1911

Family Hypopomidae Eigenmann 1912

Family Sternopygidae Cope 1871

Family Apteronotidae Jordan 1923

Order Siluriformes

Family †Vorhisiidae Frizzell 1965

$\uparrow$ Vorhisiidae Frizzell 1965: 179 (family) †Vorhisia Frizzell 1965

Family $\uparrow$ Andinichthyidae Gayet 1988

$\uparrow$ Andinichthyidae Gayet 1988: 833 (family) †Andinichthys Gayet 1988

Suborder Diplomystoidei

Family $\uparrow$ Bachmanniidae Azpelicueta \& Cione 2011

$\uparrow$ Bachmanniidae Azpelicueta \& Cione 2011: 259 (family) †Bachmannia Dolgopol 1941

Family Diplomystidae Eigenmann 1890 


\section{Suborder $\uparrow$ Hypsidoroidei}

Family $\uparrow$ Hypsidoridae Grande 1987

$\dagger$ Hypsidoridae Grande 1987: 28 (family) †Hypsidoras Lundberg \& Case 1970 [family name sometimes seen as $\dagger$ Hypsidorididae]

\section{Suborder Cetopsoidei}

Family Cetopsidae Bleeker 1858

\section{Suborder Loricarioidei}

Family Trichomycteridae Bleeker 1858

Family Nematogenyidae Bleeker 1862

Family Callichthyidae Bonaparte 1835

Family Scoloplacidae Bailey \& Baskin 1976

Family Astroblepidae Bleeker 1862

Family Loricariidae Rafinesque 1815

\section{Suborder Siluroidei}

Family Siluridae Rafinesque 1815

Family Kryptoglanidae Britz, Kakkassery \& Raghavan 2014

Family Austroglanididae Mo 1991

Family Pangasiidae Bleeker 1858

Family Chacidae Bleeker 1858

Family Plotosidae Bleeker 1858

Family Ritidae Bleeker 1862

Family Ailiidae Bleeker 1858

Family Horabagridae Jarayam 2006

Family Bagridae Bleeker 1858 [ICZN Opinion 1402]

Family Akysidae Gill 1861

Family Amblycipitidae Day 1873

Family Sisoridae Bleeker 1858

Family Amphiliidae Regan 1911

Family Malapteruridae Bleeker 1858

Family Mochokidae Regan 1912

Family Schilbeidae Bleeker 1858

Family Auchenoglanididae Jarayam 1966

Family Claroteidae Bleeker 1862

Family Lacantuniidae Rodiles-Hernández, Hendrickson \& Lundberg 2005

Family Clariidae Bonaparte 1845

Family Heteropneustidae Hora 1936

Family Anchariidae Glaw \& Vences 1994

Family Ariidae Bleeker 1858

Torpedinidae Gill 1896b: 162 (family) Torpedo Forsskål 1775 [preoccupied by Torpedines Henle 1834 in fishes; not to be used, Art. 55.3; Recent, omitted in Van der Laan et al. 2014]

Family Aspredinidae Adams 1854

Family Doradidae Bleeker 1858

Family Auchenipteridae Bleeker 1862

Family Cranoglanididae Myers 1931

Family $\uparrow$ Astephidae Grande \& Lundberg 1988

†Astephinae Grande \& Lundberg 1988: 146 (subfamily) †Astephus Cope 1873

Family Ictaluridae Gill 1861

Family Heptapteridae Gill 1861

Family Phreatobiidae Reichel 1927

Family Pimelodidae Bonaparte 1835

Family Pseudopimelodidae Fernández-Yépez \& Antón 1966 


\section{Subcohort EUTELEOSTEOMORPHA}

Incertae sedis:

$\dagger$ Erichalcidae ?? (family) †Erichalcis Forey 1973 [correct stem would be Erichalcid-] [author / date not found]

\section{Infracohort Lepidogalaxii}

Order Lepidogalaxiiformes

Family Lepidogalaxiidae Rosen 1974

\section{Infracohort Protacanthopterygii}

Incertae sedis:

$\dagger$ Bernardichthyidae Huddleston 1981: 38 (family) †Bernardichthys Huddleston 1981

Family $\uparrow$ Ferrifronsidae Arratia \& Chorn 1998

$\uparrow$ Ferrifronsidae Arratia \& Chorn 1998: 302 (family) $\uparrow$ Ferrifrons Jordan 1925

Family †Orthogonikleithridae Arratia 1997

†Orthogonikleithridae Arratia 1997: 89 (family) †Orthogonikleithrus Arratia 1987

\section{Order Argentiniformes}

Family $\uparrow$ Pattersonellidae Taverne 1982

$\dagger$ Pattersonellidae Taverne 1974: 57, 58 (family) †Pattersonella Taverne 1982 [no valid type genus, not available, Art. 11.7.1.1]

$\dagger$ Pattersonellidae Taverne 1975: 488 (family) $\uparrow$ Pattersonella Taverne 1982 [no valid type genus, not available, Art. 11.7.1.1]

$\dagger$ Pattersonelloidea Taverne 1982: 1 (superfamily) $†$ Pattersonella Taverne 1982

Family Bathylagidae Gill 1884

Family Microstomatidae Bleeker 1859

Family Opisthoproctidae Schmidt 1918

Family Argentinidae Bonaparte 1846

\section{Order Galaxiiformes}

Family Galaxiidae Müller 1845

\section{Order Esociformes}

Family $\uparrow$ Palaeoesocidae Berg 1940

$\uparrow$ Palaeoesocidae Berg 1940: 430 (family) †Palaeoesox Voigt 1934 [family name sometimes seen as $\dagger$ Palaesocidae]

Family Esocidae Rafinesque 1815

Family Umbridae Bonaparte 1845

\section{Order Salmoniformes}

Family Salmonidae Jarocki or Schinz 1822

\section{Infracohort Stomiati}

Order Stomiiformes

Suborder Gonostomatoidei

Family Gonostomatidae Cocco 1838

Suborder Stomioidei

Family Phosichthyidae Weitzman 1974

Family Sternoptychidae Duméril 1805

Family Stomiidae Bleeker 1859 


\section{Order Osmeriformes}

Incertae sedis:

Family $\uparrow$ Spaniodontidae Jordan 1905

$\uparrow$ Spaniodontidae Jordan 1905: 43 (family) †Spaniodon Pictet 1850

\section{Suborder Osmeroidei}

Family Osmeridae Regan 1913

Family Plecoglossidae Bleeker 1859

Family Salangidae Bleeker 1859

\section{Suborder Retropinnoidei}

Family Retropinnidae Gill 1862

\section{Infracohort NEOTELEOSTEI}

Incertae sedis:

$\uparrow$ Protostomiatidae Arambourg 1955: 91 (family) †Protostomias Arambourg 1943 [family name sometimes seen as $\uparrow$ Protostomiidae or $\uparrow$ Protostomatidae]

$\uparrow$ Thaumaturidae Voigt 1934: 42 (family) †Thaumaturus Reuss 1844

$\uparrow$ Tomognathidae Jordan 1923: 126 (family) †Tomognathus Agassiz 1850

\section{Order Ateleopodiformes}

Family Ateleopodidae Bonaparte 1850

\section{Supersection EURYPTERYGIA}

Incertae sedis:

Family $\uparrow$ Cheirothricidae Woodward 1901

$\uparrow$ Chirothricidae Woodward 1901: 279 (family) $†$ Cheirothrix Pictet \& Humbert 1866 [type genus as $\uparrow$ Chirothrix, name must be corrected Art. 32.5.3; corrected to $\uparrow$ Cheirothricidae by Jordan 1923: 153; family name sometimes seen as $\uparrow$ Chirothrichidae]

Section Cyclosquamata

\section{Order Aulopiformes}

Incertae sedis:

Family $\uparrow$ Nardorexidae Taverne 2004

$\uparrow$ Nardorexidae Taverne 2004a: 29 (family) †Nardorex Taverne 2004 [family name sometimes seen as $\uparrow$ Nardorecidae]

\section{Suborder $\uparrow$ Ichthyotringoidei}

\section{Family $\uparrow$ Ichthyotringidae Harry 1953}

$\uparrow$ Rhinellidae Jordan 1905: 134 (family) †Rhinellus Agassiz 1844 [type genus preoccupied by Rhinellus Bonaparte 1831 in Amphibia; invalid, Art. 39]

$†$ Ichthyotringidae Harry 1953: 243 (family) †Ichthyotringa Cope 1878

$\uparrow$ Apateopholidae Goody 1969b: 30 (family) †Apateopholis Woodward 1891 [family name sometimes seen as $\uparrow$ Apateopholididae]

Family $\uparrow$ Dercetidae von der Marck 1863

$\dagger$ Dercetiformes von der Marck 1863: 58 (family) †Dercetis Agassiz 1834 [family name sometimes seen as $\dagger$ Dercetididae]

$\uparrow$ Hoplopleurides Pictet \& Humbert 1866: 90 (family) ? †Dercetis Agassiz 1834 [published not in latinized form before 1900, not available, Art. 11.7.2; also no stem of type genus]

$\dagger$ Stratodontidae Cope 1872: 348 (family) $\uparrow$ Stratodus Cope 1872

$†$ Hoplopleuridae Günther 1880: 665 (family) ? †Dercetis Agassiz 1834 [no stem of the type genus, not available, Art. 11.7.1.1] 
Family $\uparrow$ Prionolepididae Goody 1969

$\dagger$ Prionolepididae Goody 1969b: 178 (family) †Prionolepis Egerton 1850

\section{Suborder $\uparrow$ Halecoidei}

Family $\dagger$ Halecidae Agassiz 1844

$\dagger$ Halécoides Agassiz 1844 Tome V pt. 1: 13 (family) †Halec Agassiz 1844 [latinized to $\dagger$ Halecoides by Giebel 1846: 160, 298; latinized to $\uparrow$ Halecoidei by Giebel 1847: 120; latinized to $†$ Halecidae by Goody 1969b: 126; considered valid with this authorship by Owen 1840: 5 and by Pander 1856: 12 Art. 11.7.2]

$\dagger$ Serrilepidae Chalifa 1989: 11 (family) $†$ Serrilepis Chalifa 1989 [family name sometimes seen as $\dagger$ Serrilepididae]

\section{Suborder Aulopoidei}

Family Synodontidae Gill 1861

Family Aulopidae Bonaparte 1831

Family Pseudotrichonotidae Yoshino \& Araga 1975

\section{Suborder Paraulopoidei}

Family Paraulopidae Sato \& Nakabo 2002

Suborder Alepisauroidei

Incertae sedis:

$†$ Polymerichthyidae Uyeno 1967: 384 (family) †Polymerichthys Uyeno 1967

Family Ipnopidae Gill 1884

Family Bathysauropsidae Sato \& Nakabo 2002

Family Giganturidae Brauer 1906

Family Bathysauroididae Sato \& Nakabo 2002

Family Bathysauridae Fowler 1944

Family Chlorophthalmidae Garman 1899

Family Notosudidae Parr 1928

Family Scopelarchidae Alcock 1896

Family Evermannellidae Fowler 1901

Family Sudidae Regan 1911

Family Paralepididae Bonaparte 1835

$\uparrow$ Holosteinae Prokofiev 2005: 293 (subfamily) $\uparrow$ Holosteus Agassiz 1835

Family $\dagger$ Enchodontidae Lydekker 1889, name in prevailing recent practice

$\dagger$ Saurorhamphoidei Bleeker 1859: XIV (family) †Saurorhamphus Heckel 1850

$\dagger$ Enchodontidae Lydekker in Nicholson \& Lydekker 1889: 997 (family) †Enchodus Agassiz 1835 [family name sometimes seen as $\dagger$ Enchodidae]

$\uparrow$ Cimolichthyidae Goody 1969b: 36 (family) †Cimolichthys Leidy 1857 [family name sometimes seen as $\uparrow$ Cymolichthyidae]

$\dagger$ Eurypholidae Goody 1969b: 99 (family) $†$ Eurypholis Pictet 1850 [family name sometimes seen as $\dagger$ Eurypholididae]

$\dagger$ Rharbichthinae Fielitz 2004: 631 (subfamily) †Rharbichthys Arambourg 1955 [correct stem would be Rharbichthy-]

$†$ Palaeolycinae Fielitz 2004: 632 (subfamily) †Palaeolycus von der Marck 1863

Family Alepisauridae Swainson 1839

Family Lestidiidae Harry 1953

Section Ctenosquamata

Subsection Myctophata

\section{Order Myctophiformes}

Family Neoscopelidae Jordan 1901

$\uparrow$ Sardinioididae Goody 1969b: 153 (family) †Sardinioides von der Marck 1858 
$\uparrow$ Neocassandridae Prokofiev 2002: 64 (family) $\uparrow N e o c a s s a n d r a$ Daniltshenko 1968

Family Myctophidae Gill 1893

$†$ Eomyctophinae Prokofiev 2006: S57 (subfamily) †Eomyctophum Daniltshenko 1947

Subsection Acanthomorphata

Incertae sedis:

Family $\uparrow$ Asineopidae Cope 1877

$\uparrow$ Asineopidae Cope 1877c: 570 (family) $\uparrow$ Asineops Cope 1870

Family $\uparrow$ Bajaichthyidae Bannikov \& Sorbini 2014

$\uparrow$ Bajaichthyidae Bannikov \& Sorbini in Bannikov 2014b: 625 (family) †Bajaichthys Sorbini 1983

Family $\uparrow$ Pateropercidae Gaudant 1978

$\dagger$ Pateropercidae Gaudant 1978a: 189 (family) †Pateroperca Woodward 1942

Family $\uparrow$ Pattersonichthyidae Gaudant 1976

$\uparrow$ Pattersonichthyidae Gaudant 1976: 1629 (family) $†$ Pattersonichthys Goody 1969 [described in more detail by Gaudant 1978c: 83]

\section{Order $\uparrow$ Ctenothrissiformes}

Family $\uparrow$ Ctenothrissidae Woodward 1901

$\dagger$ Ctenothrissidae Woodward 1901: 119 (family) †Ctenothrissa Woodward 1899

$\uparrow$ Aulolepidae Patterson 1964: 247 (family) †Aulolepis Agassiz 1844 [family name sometimes seen as $\uparrow$ Aulolepididae]

\section{Division Lampripterygii}

Family $\uparrow$ Pycnosteroididae Patterson 1964

$\uparrow$ Pycnosteroididae Patterson 1964: 389 (family) $\uparrow$ Pycnosteroides Woodward 1942

Family $\uparrow$ Pharmacichthyidae Patterson 1964

$\uparrow$ Pharmacichthyidae Patterson 1964: 362, 398 (family) †Pharmacichthys Woodward 1942

Family $\uparrow$ Aipichthyidae Patterson 1964

$\uparrow$ Aipichthyidae Patterson 1964: 303 (family) †Aipichthys Steindachner 1860

Family $\uparrow$ Aipichthyoididae Gayet 1980

$\uparrow$ Aipichthyoididae Gayet 1980a: 108 (family) $\uparrow$ Aipichthyoides Gayet 1980

\section{Order Lampriformes}

Family $\uparrow$ Turkmenidae Daniltshenko 1968

$\dagger$ Turkmenidae Daniltshenko 1968: 127 (family) †Turkmene Daniltshenko 1968

Family Veliferidae Bleeker 1859

Metaveliferinae Bannikov 1991c: 162 (subfamily) Metavelifer Walters 1960 [Recent, omitted in

Van der Laan et al. 2014; subfamily name sometimes seen as Metavelifrinae]

Family $\uparrow$ Palaeocentrotidae Bonde 1966

$\uparrow$ Palaeocentrotidae Bonde 1966: 200 (family) †Palaeocentrotus Kühne 1941

Family Lophotidae Bonaparte 1845

Family Lampridae Gill 1862

Family Radiicephalidae Osório 1917

Family Trachipteridae Swainson 1839

Family Regalecidae Gill 1884

\section{Division Paracanthopterygii}

Incertae sedis:

$\uparrow$ Protosyngnathidae Boulenger 1902a: 151 (family) †Protosyngnathus von der Marck 1878

$\dagger$ Trebicianiidae Sorbini \& Bannikov 1996: 47 (family) †Trebiciania Sorbini \& Bannikov 1996 
Order $\uparrow$ Sphenocephaliformes

Family $\uparrow$ Sphenocephalidae Patterson 1964

$†$ Sphenocephalidae Patterson 1964: 383 (family) †Sphenocephalus Agassiz 1838

\section{Order Percopsiformes}

Family Percopsidae Agassiz 1850

$\dagger$ Erismatopteridae Jordan 1905: 242 (family) †Erismatopterus Cope 1870

$\dagger$ Libotoniidae Grande 1988: 122, 128 (family) †Libotonius Wilson 1977

Family $\uparrow$ Mcconichthyidae Grande 1988

$\dagger$ Mcconichthyidae Grande 1988: 119 (family) $†$ Mcconichthys Grande 1988

Family Aphredoderidae Bonaparte 1845

Family Amblyopsidae Bonaparte 1845

\section{Order Zeiformes}

Suborder Cyttoidei

Family †Cretazeidae Tyler, Bronzi \& Ghiandoni 2000

$\uparrow$ Cretazeidae Tyler, Bronzi \& Ghiandoni 2000: 12 (family) †Cretazeus Tyler, Bronzi \& Ghiandoni 2000

Family †Archaeozeidae Tyler \& Santini 2005

$\uparrow$ Archaeozeidae Tyler \& Santini 2005: 166 (family) †Archaeozeus Bonde \& Tyler 2000

Family $\uparrow$ Protozeidae Tyler \& Santini 2005

$†$ Protozeidae Tyler \& Santini 2005: 166 (family) †Protozeus Bonde \& Tyler 2000

Family Cyttidae Günther 1860

\section{Suborder Zeoidei}

Family Oreosomatidae Bleeker 1859

Family Zeniontidae Myers 1960

Family Parazenidae McAllister 1968

$\dagger$ Isozenidae Schwarzhans 1996: 427 (family) †Isozen Schwarzhans 2010 [no valid type genus, not available, Art. 11.7.1.1; also name only, published after 1960, not available, Art. 13.1.1]

Family Grammicolepididae Poey 1873

Family Zeidae Rafinesque 1815

\section{Order Stylephoriformes}

Family Stylephoridae Swainson 1839

\section{Order Gadiformes}

Suborder Melanonoidei

Family Melanonidae Goode \& Bean 1896

Suborder Macrouroidei

Family Steindachneriidae Parr 1942

Family Bathygadidae Jordan \& Evermann 1898

Family Macrouridae Bonaparte 1831

Family Trachyrincidae Goode \& Bean 1896

\section{Suborder Gadoidei}

Family Muraenolepididae Regan 1903

Family Euclichthyidae Cohen 1984

Family Moridae Moreau 1881

Family Macruronidae Regan 1903

Family Merlucciidae Rafinesque 1815

Family Ranicipitidae Bonaparte 1835

Family Bregmacerotidae Gill 1872 
Family Phycidae Swainson 1838

Family Gaidropsaridae Jordan \& Evermann 1898

Family Lotidae Bonaparte 1835

Family Gadidae Rafinesque 1810

\section{Division Polymixiipterygii}

Order Polymixiiformes

Family $\uparrow$ Dinopterygidae Jordan 1923

$\dagger$ Dinopterygidae Jordan 1923: 173 (family) †Dinopteryx Woodward 1901 [family name sometimes seen as $\dagger$ Dinopterygiidae]

Family $\uparrow$ Digoriidae Bannikov \& Daniltshenko 1985

$\dagger$ Digoriidae Bannikov \& Daniltshenko 1985: 197 (family) $†$ Digoria Daniltshenko 1980

Family $\uparrow$ Boreiohydriidae Murray \& Cumbaa 2013

$\uparrow$ Boreiohydriidae Murray \& Cumbaa 2013: 294 (family) $†$ Boreiohydrias Murray \& Cumbaa 2013

Family Polymixiidae Bleeker 1859

$\uparrow$ Berycopsidae Regan 1911: 4 (family) †Berycopsis Dixon 1850

$\uparrow$ Homonotichthyidae Whitley 1933: 146 (family) $†$ Homonotichthys Whitley 1933

†Dalmatichthyidae Radovčić 1975: 35 (family) †Dalmatichthys Radovčić 1975

†Omosomopsidae Gaudant 1978b: 86 (family) †Omosomopsis Gaudant 1978

\section{Division Acanthopterygii}

Subdivision Berycimorphaceae

Order Beryciformes

Suborder Berycoidei

Family $\uparrow$ Quaesitoberycidae Bannikov \& Sorbini 2005

$\uparrow$ Quaesitoberycidae Bannikov \& Sorbini 2005: 29 (family) $\uparrow Q u a e s i t o b e r y x$ Bannikov \& Sorbini 2005

Family Berycidae Lowe 1839

Family Melamphaidae Gill 1893

Suborder Stephanoberycoidei

Family Gibberichthyidae Par 1933

Family Hispidoberycidae Kotlyar 1981

Family Rondeletiidae Goode \& Bean 1895

Family Stephanoberycidae Gill 1884

Family Barbourisiidae Parr 1945

Family Cetomimidae Goode \& Bean 1895

\section{Order Trachichthyiformes}

Family Diretmidae Gill 1893

Family Anoplogastridae Gill 1893

Family Trachichthyidae Bleeker 1856

$\dagger$ Hoplopterygidae Cockerell 1919: 186 (family) †Hoplopteryx Agassiz 1838 [family name sometimes seen as $\dagger$ Hoplopterygiidae]

$\dagger$ Lissoberycinae Gayet 1980b: 99 (subfamily) †Lissoberyx Patterson 1967

Family Anomalopidae Gill 1889

Family †Pseudomonocentrididae González-Rodríguez, Schultze \& Arratia 2013

$†$ Pseudomonocentrididae González-Rodríguez, Schultze \& Arratia 2013: 466 (family) $\dagger$ Pseudomonocentris González-Rodríguez, Schultze \& Arratia 2013 [family name sometimes seen as $\uparrow$ Pseudomonocentridae]

Family Monocentridae Gill 1859 
Subdivision Holocentrimorphaceae

Order Holocentriformes

Family $\uparrow$ Tenuicentridae Gayet 1982

$\uparrow$ Tenuicentrinae Gayet 1982a: 28 (subfamily) †Tenuicentrum Sorbini 1975

Family $\uparrow$ Caproberycidae Patterson 1967

$\uparrow$ Caproberycinae Patterson 1967: 88 (subfamily) †Caproberyx Regan 1911

$\uparrow$ Alloberycinae Gayet 1982a: 28 (subfamily) $\uparrow$ Alloberyx Gaudant 1969

$\uparrow$ Stichocentridae Gayet 1982a: 32 (family) †Stichocentrus Patterson 1967 [name only, published after 1960, not available, Art. 13.1.1]

$\uparrow$ Stichocentridae Gayet 1982b: 99 (family) †Stichocentrus Patterson 1967

Family Holocentridae Bonaparte 1833

Subdivision Percomorphaceae

Series Ophidiaria

Order Ophidiiformes

Suborder Ophidioidei

Family Ophidiidae Rafinesque 1810

Hypopleuroninae Prokofiev 2004: 38 (subfamily) Hypopleuron Smith \& Radcliffe 1913 [Recent, omitted in Van der Laan et al. 2014]

\section{Suborder Bythitoidei}

Family Dinematichthyidae Whitley 1928

Family Bythitidae Gill 1861

Series Batrachoidaria

Order Batrachoidiformes

Family Batrachoididae Jordan 1896 (1835)

Series Pelagiaria

Order Scombriformes

Family Amarsipidae Haedrich 1969

Family Ariommatidae Haedrich 1967

Family Arripidae Gill 1893

Family Bramidae Bonaparte 1831

Family †Carangodidae Blot 1969

$\uparrow$ Carangodidae Blot 1969: 426 (family) †Carangodes Heckel 1856 [senior objective synonym of $\uparrow$ Arambourgellidae Blot 1981]

$\uparrow$ Arambourgellidae Blot 1981: 377 (family) $\uparrow$ Arambourgella Blot 1981 [junior objective synonym of $\dagger$ Carangodidae Blot 1969, invalid, Art. 61.3.2]

Family Caristiidae Gill \& Smith 1905

Family Centrolophidae Bonaparte 1846

Family Chiasmodontidae Jordan \& Gilbert 1883

Family $†$ Euzaphlegidae Daniltshenko 1960

$†$ Zaphlegidae Jordan \& Gilbert 1920: 23 (family) †Zaphleges Jordan 1920 [type genus preoccupied by Zaphleges Foerster 1868 in Hymenoptera; invalid, Art. 39]

$\dagger$ Euzaphlegidae Daniltshenko 1960: 135 (family) †Euzaphleges White \& Moy-Thomas 1941 [family name sometimes seen as $†$ Eusaphlegidae]

†Dipterichthyidae Arambourg 1967: 111 (family) †Dipterichthys Arambourg 1967

Family Gempylidae Gill 1862

Family Icosteidae Jordan \& Gilbert 1880 
VAN DER LAAN R., Family-group names of fossil fishes

Family Nomeidae Günther 1860

Family Pomatomidae Gill 1863

Family $\uparrow$ Propercarinidae Bannikov 1995

†Propercarinidae Bannikov 1995: 179 (family) †Propercarina Paucă 1929

Family Scombridae Rafinesque 1815

$†$ Eocoelopomini Monsch \& Bannikov 2012: 277 (tribe) †Eocoelopoma Woodward 1901

Family Scombrolabracidae Fowler 1925

Family Scombropidae Gill 1862

Family Stromateidae Rafinesque 1810

Family Tetragonuridae Risso 1827

Family Trichiuridae Rafinesque 1810

$\uparrow$ Anenchelini Bonaparte 1850b (subfamily) †Anenchelum de Blainville 1818 [type genus inferred from the stem, Art. 11.7.1.1]

Series Syngnatharia

Order Syngnathiformes

Incertae sedis:

Family $\uparrow$ Aulorhamphidae Tyler 2004

$\uparrow$ Aulorhamphidae Tyler 2004: 37 (family) $\uparrow$ Aulorhamphus de Zigno 1887

Family $\uparrow$ Gerpegezhidae Bannikov \& Carnevale 2012

$\uparrow$ Gerpegezhidae Bannikov \& Carnevale 2012: 381 (family) †Gerpegezhus Bannikov \& Carnevale 2012

Family $\uparrow$ Paraeoliscidae Blot 1981

$†$ Paraeoliscidae Blot 1981: 361 (family) †Paraeoliscus Blot 1981

Family $\uparrow$ Rhamphosidae Gill 1884

$\dagger$ Rhamphosidae Gill 1884: 165 (family) †Rhamphosus Agassiz 1844 [family name sometimes seen as $\uparrow$ Ramphosidae]

Family †Urosphenidae Gill 1884

†Urosphenidae Gill 1884: 165 (family) †Urosphen Agassiz 1844

\section{Suborder Dactylopteroidei}

Family †Pterygocephalidae Clark Hubbs 1952

$\uparrow$ Pterygocephalidae Clark Hubbs 1952: 50 (family) †Pterygocephalus Agassiz 1839 [also as a new family in Blot 1981: 367]

Family Dactylopteridae Gill 1861

Family Pegasidae Bonaparte 1831

\section{Suborder Callionymoidei}

Family Callionymidae Bonaparte 1831

Family Draconettidae Jordan \& Fowler 1903

\section{Suborder Mulloidei}

Family Mullidae Rafinesque 1815

\section{Suborder Syngnathoidei}

Family †Eekaulostomidae Cantalice \& Alvarado-Ortega 2016

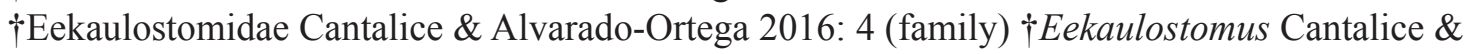
Alvarado-Ortega 2016

Family Fistulariidae Stark 1828

$\uparrow$ Parasynarcualidae Blot 1981: 363 (family) $\uparrow$ Parasynarcualis Blot 1981

$\uparrow$ Fistularioididae Blot 1981: 363 (family) $\uparrow$ Fistularioides Blot 1981

Family Aulostomidae Rafinesque 1815

Family Centriscidae Bonaparte 1831 
Family Solenostomidae Nardo 1843

$\dagger$ Solenorhynchinae Bannikov \& Carnevale 2017: 320 (subfamily) †Solenorhynchus Heckel 1854

Family Syngnathidae Bonaparte 1831

$\dagger$ Eogastrophinae Jerzmańska 1969: 436 (subfamily) †Hipposyngnathus Daniltshenko 1960 [no stem of the type genus, not available, Art. 11.7.1.1]

$\dagger$ Hipposyngnathinae Fritzsche 1980: 216 (subfamily) $†$ Hipposyngnathus Daniltshenko 1960

$†$ Pshekhagnathinae Bannikov, Carnevale \& Popov 2017: 80 (subfamily) †Pshekhagnathus Bannikov, Carnevale \& Popov 2017

Series Gobiaria

Order Kurtiformes

Suborder Kurtoidei

Family Kurtidae Bleeker 1859

Suborder Apogonoidei

Family Apogonidae Günther 1859

$\dagger$ Eoapogonini Bannikov 2005: 120 (tribe) †Eoapogon Bannikov 2005

\section{Order Gobiiformes}

Suborder Trichonotoidei

Family Trichonotidae Günther 1861

Suborder Gobioidei

Family Rhyacichthyidae Jordan 1905

Family Odontobutidae Hoese \& Gill 1993

Family Milyeringidae Whitley 1945

Family Eleotridae Bonaparte 1835

†Pirskeniidae Obrhelová 1961: 107 (family) †Pirskenius Obrhelová 1961

Family Butidae Bleeker 1874

Family Thalasseleotrididae Gill \& Mooi 2012

Family Oxudercidae Günther 1861

Family Gobiidae Cuvier 1816

Series Anabantaria

\section{Order Synbranchiformes}

Suborder Mastacembeloidei

Family Mastacembelidae Swainson 1839

Family Chaudhuriidae Annandale 1918

Suborder Indostomoidei

Family Indostomidae Prashad \& Mukerji 1929

Suborder Synbranchoidei

Family Synbranchidae Bonaparte 1835

\section{Order Anabantiformes}

Incertae sedis:

Family $\uparrow$ Nardoichthyidae Sorbini \& Bannikov 1991

$\uparrow$ Nardoichthyidae Sorbini \& Bannikov 1991: 240 (family) $\uparrow$ Nardoichthys Sorbini \& Bannikov 1991

\section{Suborder Anabantoidei}

Family Anabantidae Bonaparte 1831

Family Helostomatidae Gill 1872

Family Osphronemidae van der Hoeven 1832 


\section{Suborder Channoidei}

Family Channidae Fowler 1934 (1831)

Suborder Nandoidei

Family Pristolepididae Regan 1913

Family Nandidae Bleeker 1852

Family Badidae Barlow, Liem \& Wickler 1968

Series Carangaria

Incertae sedis:

†Pygaeidae Jordan 1905: 405 (family) †Pygaeus Agassiz 1838

Family Centropomidae Poey 1867

Family Lactariidae Boulenger 1904

Family Leptobramidae Ogilby 1913

Family Menidae Fitzinger 1873

Family Polynemidae Rafinesque 1815

Family Sphyraenidae Rafinesque 1815

Family Toxotidae Bleeker 1859

\section{Order Istiophoriformes}

Family $\uparrow$ Palaeorhynchidae Günther 1880

$\uparrow$ Palaeorhynchidae Günther 1880: 437 (family) †Palaeorhynchus de Blainville 1818

$\uparrow$ Aglyptorhynchinae Fierstine \& Weems 2009: 84 (subfamily) †Aglyptorhynchus Casier 1966

Family $\uparrow$ Hemingwayidae Sytchevskaya \& Prokofiev 2002

$\dagger$ Hemingwayidae Sytchevskaya \& Prokofiev 2002: 227 (family) $†$ Hemingwaya Sytchevskaya \& Prokofiev 2002

Family Istiophoridae Rafinesque 1815

Family Xiphiidae Rafinesque 1815

$\uparrow$ Xiphiorhynchidae Regan 1909: 75 (family) †Xiphiorhynchus van Beneden 1871

Family $\uparrow$ Blochiidae Bleeker 1859

$\dagger$ Rostrati Giebel 1847: 156 (family) †Blochius Volta 1796 [no stem of the type genus, not available, Art. 11.7.1.1]

$\dagger$ Blochioides Pictet 1854: 124 (family) †Blochius Volta 1796 [published not in latinized form before 1900, not available, Art. 11.7.2]

$\uparrow$ Blochioidei Bleeker 1859: XVI (family) $\uparrow$ Blochius Volta 1796

\section{Order Carangiformes}

Family Nematistiidae Gill 1862

Family Carangidae Rafinesque 1815

†Vomeropsinae Bannikov 1984: 319 (subfamily) †Vomeropsis Heckel 1854

$\uparrow$ Archaeinae Bannikov 1990: 14 (subfamily) †Archaeus Agassiz 1844 [family-group name preoccupied by Archaeidae Koch \& Berendt 1854 in Arachnida spiders, not to be used, Art. 55.3]

$\uparrow$ Paratrachinotini Springer \& Smith-Vaniz 2008: 1 (tribe) $\uparrow$ Paratrachinotus Blot 1969

Family $\uparrow$ Ductoridae Blot 1969

$\uparrow$ Ductoridae Blot 1969: 95 (family) $†$ Ductor Agassiz 1844

Family Rachycentridae Gill 1896

Family Coryphaenidae Rafinesque 1815

Family †Opisthomyzonidae Jordan 1923

†Opisthomyzonidae Jordan 1923: 227 (family) †Opisthomyzon Cope 1889 [family name sometimes seen as †Opisthomyzontidae or †Opisthomyzidae]

Family Echeneidae Rafinesque 1810 
Order Pleuronectiformes

Family $\uparrow$ Amphistiidae Boulenger 1902

Suborder Psettodoidei

$\dagger$ Amphistiidae Boulenger 1902b: 300 (family) †Amphistium Agassiz 1835

Family Psettodidae Regan 1910

Suborder Pleuronectoidei

Family †Joleaudichthyidae Chabanaud 1937

$\dagger$ Joleaudichthyidae Chabanaud 1937: 51 (family) †Joleaudichthys Chabanaud 1937

Family Citharidae de Buen 1935

Eucitharidae Romer 1966: 361 (family) Eucitharus Gill 1889 [name only, published after 1960, not available, Article 13.1.1; Recent, omitted in Van der Laan et al. 2014]

Family Paralichthyidae Regan 1910

Family Pleuronectidae Rafinesque 1815

Family Bothidae Smitt 1892

Family Paralichthodidae Regan 1920

Family Scophthalmidae Chabanaud 1933

Family Rhombosoleidae Regan 1910

Family Achiropsettidae Heemstra 1990

Family Achiridae Rafinesque 1815

Family Samaridae Jordan \& Goss 1889

Family Poecilopsettidae Norman 1934

Family Cynoglossidae Jordan 1888

Family Soleidae Bonaparte 1833

$\dagger$ Eobuglossidae Chabanaud 1937: 73 (family) $†$ Eobuglossus Chabanaud 1931

Series Ovalentaria

Incertae sedis:

Family Ambassidae Klunzinger 1870

Family Congrogadidae Günther 1862

Family Embiotocidae Agassiz 1853

Family Grammatidae Jordan 1887

Family Opistognathidae Bonaparte 1835

Family Plesiopidae Günther 1861

Family Polycentridae Gill 1858

Family Pomacentridae Bonaparte 1831

Family Pseudochromidae Müller \& Troschel 1849

\section{Superorder Cichlomorphae}

Order Cichliformes

Incertae sedis:

Family $\uparrow$ Priscacaridae Jordan 1923

$\uparrow$ Priscacaridae Jordan 1923: 218 (family) †Priscacara Cope 1877

Family Cichlidae Bonaparte 1835

Family Pholidichthyidae Jordan 1896

\section{Superorder Atherinomorphae}

Incertae sedis:

Family $\uparrow$ Mesogasteridae Bannikov 2008

$\dagger$ Mesogasteridae Bannikov 2008: 83 (family) $†$ Mesogaster Agassiz 1844 
Family $\uparrow$ Rhamphognathidae Bannikov 2008

$\dagger$ Rhamphognathidae Bannikov 2008: 78 (family) $†$ Rhamphognathus Agassiz 1844

\section{Order Atheriniformes}

Suborder Atherinopsoidei

Family Atherinopsidae Fitzinger 1873

Suborder Atherinoidei

Family Isonidae Rosen 1964

Family Bedotiidae Jordan \& Hubbs 1919

Family Melanotaeniidae Gill 1894

Family Pseudomugilidae Kner 1867

Family Telmatherinidae Munro 1958

Family Atherionidae Schultz 1948

Family Dentatherinidae Patten \& Ivantsoff 1983

Family Phallostethidae Regan 1916

Family Atherinidae Risso 1827

\section{Order Beloniformes}

Family $\uparrow$ Cobitopsidae Jordan 1905

$\uparrow$ Cobitopsidae Jordan 1905: 224 (family) $†$ Cobitopsis Pomel 1853

Family $\uparrow$ Forficidae Jordan \& Gilbert 1919

$\uparrow$ Forficidae Jordan \& Gilbert 1919: 36 (family) †Forfex Jordan \& Gilbert 1919 [family name

Suborder Adrianichthyoidei sometimes seen as $\uparrow$ Forfecidae or $\dagger$ Forficiidae]

Family Adrianichthyidae Weber 1913

Suborder Belonoidei

Family Exocoetidae Risso 1827

$\uparrow$ Rhamphexocoetinae Bannikov, Parin \& Pinna 1985: 154 (subfamily) $†$ Rhamphexocoetus Bannikov, Parin \& Pinna 1985

Family Hemiramphidae Gill 1859

Family Zenarchopteridae Fowler 1934

Family Belonidae Bonaparte 1835

\section{Order Cyprinodontiformes}

Suborder Aplocheiloidei

Family Aplocheilidae Bleeker 1859

Family Nothobranchiidae Garman 1895

Family $\uparrow$ Kenyaichthyidae Altner \& Reichenbacher 2015

$\dagger$ Kenyaichthyidae Altner \& Reichenbacher 2015: 6 (family) †Kenyaichthys Altner \& Reichenbacher 2015

Family Rivulidae Myers 1925 [Case 3747 ICZN; also seen as Cynolebiidae]

\section{Suborder Cyprinodontoidei}

Family Pantanodontidae Myers 1955

Family Cyprinodontidae Wagner 1828

Family Fundulidae Günther 1866

Family Orestiidae Bleeker 1859

Family Profundulidae Hoedeman \& Bronner 1951

Family Goodeidae Jordan \& Gilbert 1883

Family Valenciidae Parenti 1981

Family Aphaniidae Hoedeman 1949 
Family Procatopodidae Fowler 1916

Family Fluviphylacidae Roberts 1970

Family Poeciliidae Bonaparte 1831

Family Anablepidae Bonaparte 1831

\section{Superorder Mugilomorphae \\ Order Mugiliformes}

Family Mugilidae Jarocki 1822

\section{Superorder Blenniomorphae \\ Order Gobiesociformes}

Family Gobiesocidae Bleeker 1859

\section{Order Blenniiformes}

Family Tripterygiidae Whitley 1931

Family Clinidae Swainson 1839

Family Dactyloscopidae Gill 1859

Family Labrisomidae Clark Hubbs 1952

Family Chaenopsidae Gill 1865

Family Blenniidae Rafinesque 1810

Series Eupercaria

Incertae sedis:

$\uparrow$ Asianthidae Sytchevskaya \& Prokofiev 2003: 2 (family) $\uparrow$ Asianthus Sytchevskaya \& Prokofiev 2003 Family Callanthiidae Ogilby 1899

Family †Caucasichthyidae Bannikov, Carnevale \& Parin 2011

$\uparrow$ Caucasichthyidae Bannikov, Carnevale \& Parin 2011: 83 (family) †Caucasichthys Bannikov, Carnevale \& Parin 2011

Family Centrogenyidae Fowler 1907

Family Dinolestidae Whitley 1948

Family Dinopercidae Heemstra \& Hecht 1986

Family Emmelichthyidae Poey 1867

Family $†$ Eotrigonodontidae White 1935

$\uparrow$ Eotrigonodontidae White 1935: 19, 56 (family) †Eotrigonodon Weiler 1929 [family name sometimes seen as $\dagger$ Eotrigonidae or $\dagger$ Eotrogonidae]

Family $†$ Exelliidae Blot 1969

$\dagger$ Semiophoridae Jordan 1905: 245 (family) $†$ Semiophorus Agassiz 1838 [type genus preoccupied by Semiophorus Wagler 1830 in Agamidae Reptilia; invalid, Art. 39]

$\dagger$ Exelliidae Blot 1969: 120 (family) †Exellia White \& Moy-Thomas 1941 [family name sometimes seen as $†$ Exellidae]

Family Malacanthidae Poey 1861

Family Monodactylidae Jordan \& Evermann 1898

Family Moronidae Jordan \& Evermann 1896

Family Parascorpididae Smith 1949

Family $\uparrow$ Pietschellidae Carnevale \& Bannikov 2015

$\uparrow$ Pietschellidae Carnevale \& Bannikov 2015: 18 (family) †Pietschellus Bannikov \& Carnevale 2011

Family Pomacanthidae Jordan \& Evermann 1898

Family $\uparrow$ Quasimullidae Bannikov 1999

†Quasimullidae Bannikov 1999: 119 (family) †Quasimullus Bannikov 1999 
Family $\uparrow$ Repropcidae Bannikov 1991

$†$ Repropcidae Bannikov 1991b: 90 (family) †Repropca Bannikov 1991

Family Scatophagidae Gill 1883

$\uparrow$ Ruffoichthyinae Bannikov \& Tyler 2002: 43 (subfamily) $\uparrow R$ uffoichthys Sorbini 1983

Family Siganidae Richardson 1837

Family Sillaginidae Richardson 1846

\section{Order Gerreiformes}

Family Gerreidae Bleeker 1859 [ICZN Opinion 962]

\section{Order Uranoscopiformes}

Family Uranoscopidae Bonaparte 1831

Family Ammodytidae Bonaparte 1835

Family Cheimarrichthyidae Regan 1913

Family Pinguipedidae Günther 1860

\section{Order Labriformes}

Family $\uparrow$ Tortonesiidae Sorbini, Boscaini \& Bannikov 1991

$\uparrow$ Tortonesidae Sorbini, Boscaini \& Bannikov 1991: 116 (family) †Tortonesia Sorbini 1983 [correct stem is Tortonesi-]

Family Labridae Cuvier 1816

$\uparrow$ Pharyngodopilidae Cocchi 1864: 25 (family) †Pharyngodopilus Cocchi 1864

$\dagger$ Trigonodontidae Arambourg 1927: 221 (family) †Trigonodon Sismonda 1847

\section{Order Ephippiformes}

Family Drepaneidae Gill 1872 [ICZN Opinion 1046]

Family Ephippidae Bleeker 1859

\section{Order Chaetodontiformes}

Family Leiognathidae Gill 1893

$†$ Eoleiognathinae Bannikov 2014a: 36 (subfamily) †Eoleiognathus Bannikov 2014

Family Chaetodontidae Rafinesque 1815

\section{Order Acanthuriformes}

Family $\uparrow$ Acanthonemidae Bannikov 1991

$\uparrow$ Acanthonemidae Bannikov 1991a: 50 (family) †Acanthonemus Agassiz 1834

Family $\dagger$ Sorbinipercidae Tyler 1998

†Sorbinipercidae Tyler 1998: 523 (family) †Sorbiniperca Tyler 1998

Family $\uparrow$ Zorzinichthyidae Tyler \& Bannikov 2002

$\nmid$ Zorzinichthyidae Tyler \& Bannikov 2002: 24 (family) †Zorzinichthys Tyler \& Bannikov 2002

Family Luvaridae Gill 1885

$\dagger$ Beerichthyidae Casier 1966: 158 (family) †Beerichthys Casier 1966

Family $\dagger$ Kushlukiidae Daniltshenko 1968

$†$ Kushlukiidae Daniltshenko 1968: 147 (family) $†$ Kushlukia Daniltshenko 1968

Family $\dagger$ Caprovesposidae Bannikov \& Fedotov 1984

$\uparrow$ Caprovesposidae Bannikov \& Fedotov 1984: 708 (family) †Caprovesposus Daniltshenko 1960

Family † Massalongiidae Tyler \& Bannikov 2005

$†$ Massalongiidae Tyler \& Bannikov 2005: 75 (family) †Massalongius Tyler \& Bannikov 2005

Family Zanclidae Bleeker 1876

Family Acanthuridae Bonaparte 1835 
Order Lutjaniformes

Family Haemulidae Gill 1885

Family Lutjanidae Gill 1861

Family Sciaenidae Cuvier 1829

†Ioscionidae David 1943: 149 (family) †Ioscion Jordan 1921 [family name sometimes seen as $\dagger$ Iosciidae]

†Carnevalellinae Bannikov 2013: 192 (subfamily) †Carnevalella Bannikov 2013

\section{Order Lobotiformes}

Family Datnioididae Fowler 1931

Family Lobotidae Gill 1861

Family Hapalogenyidae

\section{Order Spariformes}

Family Nemipteridae Regan 1913

Family Lethrinidae Bonaparte 1831

Family Sparidae Rafinesque 1818

\section{Order Priacanthiformes}

Family Cepolidae Rafinesque 1815

Family Priacanthidae Günther 1859

\section{Order Caproiformes}

Family Caproidae Bonaparte 1835

\section{Order Lophiiformes}

\section{Suborder Lophioidei}

Family Lophiidae Rafinesque 1810

\section{Suborder Antennarioidei}

Family Antennariidae Jarocki 1822

Family Tetrabrachiidae Regan 1912

Family Lophichthyidae Boeseman 1964

Family Brachionichthyidae Gill 1863

Suborder Chaunacoidei

Family Chaunacidae Gill 1863

\section{Suborder Ogcocephaloidei}

Family Ogcocephalidae Gill 1893

\section{Suborder Ceratioidei}

Family Caulophrynidae Goode \& Bean 1896

Family Neoceratiidae Regan 1926

Family Melanocetidae Gill 1878

Family Himantolophidae Gill 1861

Family Diceratiidae Regan \& Trewavas 1932

Family Oneirodidae Gill 1878

Family Thaumatichthyidae Smith \& Radcliffe 1912

Family Centrophrynidae Bertelsen 1951

Family Ceratiidae Gill 1861

Family Gigantactinidae Boulenger 1904

Family Linophrynidae Regan 1925 


\section{Order Tetraodontiformes}

\section{Suborder $\uparrow$ Plectocretacicoidei}

Family $\uparrow$ Cretatriacanthidae Tyler \& Sorbini 1996

$\dagger$ Cretatriacanthidae Tyler \& Sorbini 1996: 4 (family) †Cretatriacanthus Tyler \& Sorbini 1996

Family $\uparrow$ Plectocretacicidae Tyler \& Sorbini 1996

$\uparrow$ Plectocretacicoidea Tyler \& Sorbini 1996: 3 (superfamily) $†$ Plectocretacicus Sorbini 1979

Family $\uparrow$ Protriacanthidae Tyler \& Sorbini 1996

$\uparrow$ Protriacanthidae Tyler \& Sorbini 1996: 27 (family) †Protriacanthus d'Erasmo 1946

\section{Suborder Triacanthodoidei}

Family Triacanthodidae Gill 1862

Suborder Triacanthoidei

Family Triacanthidae Bleeker 1859

$\uparrow$ Protacanthodinae Tyler 1968: 238 (subfamily) †Protacanthodes Gill 1888

$\dagger$ Cryptobalistinae Tyler 1968: 243 (subfamily) †Cryptobalistes Tyler 1968

\section{Suborder Balistoidei}

Family †Moclaybalistidae Santini \& Tyler 2003

$\uparrow$ Moclaybalistidae Santini \& Tyler 2003: 603 (family) †Moclaybalistes Tyler \& Santini 2002

Family $\uparrow$ Bolcabalistidae Tyler \& Sorbini 1998

†Bolcabalistidae Tyler \& Sorbini 1998: 43 (family) †Bolcabalistes Tyler \& Sorbini 1998

Family $\dagger$ Eospinidae Santini \& Tyler 2003

$†$ Eospinidae Santini \& Tyler 2003: 603 (family) †Eospinus Tyler \& Bannikov 1992

Family Balistidae Rafinesque 1810

$\uparrow$ Acanthodermidae Bonaparte 1850b (family) $\uparrow$ Acanthoderma Agassiz 1844

Family Monacanthidae Nardo 1843

\section{Suborder Ostracioidei}

Family $\uparrow$ Spinacanthidae Jordan 1905

$\uparrow$ Spinacanthidae Jordan 1905: 415 (family) $\uparrow$ Spinacanthus Agassiz 1835

Family $\uparrow$ Protobalistidae Gill 1888

$†$ Protobalistidae Gill 1888: 447 (family) †Protobalistum Zigno 1887

Family Aracanidae Hollard 1860

Family Ostraciidae Rafinesque 1810

\section{Suborder Tetraodontoidei}

\section{Incertae sedis:}

Family $\uparrow$ Avitoplectidae Bemis, Tyler, Bemis, Kumar, Singh Rana \& Smith 2018

$\uparrow$ Avitoplectidae Bemis, Tyler, Bemis, Kumar, Singh Rana \& Smith 2018: [3] (family)

$\uparrow$ Avitoplectus Bemis, Tyler, Bemis, Kumar, Singh Rana \& Smith 2018

Family †Eoplectidae Tyler 1973

$†$ †oplectinae Tyler 1973: 146 (subfamily) †Eoplectus Tyler 1973

Family $\uparrow$ Zignoichthyidae Winterbottom 1974

$\dagger$ Zignoichthyidae Winterbottom 1974: 99 (family) †Zignoichthys Tyler 1973

Family Triodontidae Bleeker 1859

Family Molidae Bonaparte 1835

Family $\uparrow$ Balkariidae Bannikov, Tyler, Arcila \& Carnevale 2016

$\dagger$ Balkariidae Bannikov, Tyler, Arcila \& Carnevale 2016: [5] (family) †Balkaria Bannikov, Tyler, Arcila \& Carnevale 2016

Family Tetraodontidae Bonaparte 1831

Family Diodontidae Billberg 1833 [correction of Van der Laan et al. 2014]

Diodontiides Billberg 1833: 52 (Natio $\approx$ family) Diodon Linnaeus 1758 [Recent, omitted in Van der Laan et al. 2014] 
$\dagger$ Eodiodontidae Tavani 1955: 178 (family) †Eodiodon Casier 1952

$\uparrow$ Progymnodontinae Tavani 1955: 178 (subfamily) †Progymnodon Dames 1883

$\uparrow$ Pshekhadiodontinae Bannikov \& Tyler in Tyler \& Bannikov 2009: 703 (subfamily)

$\dagger$ Pshekhadiodon Bannikov \& Tyler 1997

\section{Order Pempheriformes}

Family Acropomatidae Gill 1893

Family Banjosidae Jordan \& Thompson 1912

Family Bathyclupeidae Gill 1896

Family Champsodontidae Jordan \& Snyder 1902

Family Creediidae Waite 1899

Family Epigonidae Poey 1861

Family Glaucosomatidae Jordan \& Thompson 1911

Family Hemerocoetidae Kaup 1873

Family Howellidae Ogilby 1899

Family Lateolabracidae

Family Leptoscopidae Gill 1859

Family Ostracoberycidae Fowler 1934

Family Pempheridae Bleeker 1859

Family Pentacerotidae Bleeker 1859

Family Polyprionidae Bleeker 1874

Family Symphysanodontidae Katayama 1984

\section{Order Centrarchiformes}

Suborder Percolatoidei

\section{Suborder Percichthyoidei}

Family Percichthyidae Jordan \& Eigenmann 1890

\section{Suborder Terapontoidei}

Family Girellidae Gill 1862

Family Dichistiidae Smith 1935

Family Kuhliidae Jordan \& Evermann 1896

Family Oplegnathidae Bleeker 1853

Family Kyphosidae Jordan 1887

Family Microcanthidae Bleeker 1876

Family Scorpididae Günther 1860

Family Terapontidae Richardson 1842

\section{Suborder Centrarchoidei}

Family Centrarchidae Bleeker 1859

Family Elassomatidae Jordan 1877

Family Enoplosidae Gill 1893

Family Sinipercidae Jordan \& Richardson 1910

\section{Suborder Cirrhitioidei}

Family Cirrhitidae Macleay 1841

Family Latridae Gill 1862

Family Cheilodactylidae Bonaparte 1850

Family Chironemidae Gill 1862

Family Aplodactylidae Günther 1859 


\section{Order Perciformes}

Incertae sedis:

Family $\uparrow$ Callipterygidae Jordan 1905

$\uparrow$ Callipterygidae Jordan 1905: 501 (family) †Callipteryx Agassiz 1838

Family $\dagger$ Eocottidae Bannikov 2004

$\dagger$ Eocottidae Bannikov 2004: 18 (family) †Eocottus Woodward 1901 [also as new subfamily]

$\uparrow$ Bassaniinae Bannikov 2004: 25 (subfamily) $†$ Bassania Bannikov 2004 [type genus preoccupied by Bassania Walker 1860 in Lepidoptera; invalid, Art. 39]

$\uparrow$ Bassanichthyinae Bannikov 2006: 340 (subfamily) †Bassanichthys Bannikov 2006

Family $\uparrow$ Robertanniidae Bannikov 2011

$\uparrow$ Robertanniidae Bannikov 2011: 8 (family) $\uparrow$ Robertannia Bannikov 2011

Family $\uparrow$ Trispinachidae Nazarkin 2002

$\uparrow$ Trispinachidae Nazarkin 2002: 419 (family) †Trispinax Nazarkin 2002

\section{Suborder Bembropoidei}

Family Bembropidae Regan 1913

Suborder Normanichthyoidei

Family Normanichthyidae Clark 1937

Suborder Serranoidei

Family Serranidae Swainson 1839

Suborder Percoidei

Family Niphonidae Jordan 1923

Family Percidae Rafinesque 1815

Family Trachinidae Rafinesque 1815

Suborder Notothenioidei

Family Bovichtidae Gill 1862

Family Pseudaphritidae McCulloch 1929

Family Eleginopsidae Gill 1893

Family Nototheniidae Günther 1861

Family Harpagiferidae Gill 1861

Family Artedidraconidae Andriashev 1967

Family Bathydraconidae Regan 1913

Family Channichthyidae Gill 1861

Family Percophidae Swainson 1839

Suborder Congiopodoidei

Family Congiopodidae Gill 1889

Suborder Scorpaenoidei

Family Sebastidae Kaup 1873

Family Setarchidae Matsubara 1943

Family Neosebastidae Matsubara 1943

Family Plectrogeniidae Fowler 1938

Family Scorpaenidae Risso 1827

Family Apistidae Gill 1859

Family Synanceiidae Swainson 1839

Family Eschmeyeridae Mandrytsa 2001

Family Perryenidae Honma, Imamura \& Kawai 2013

Family Aploactinidae Jordan \& Starks 1904

Family Pataecidae Gill 1872

Family Gnathanacanthidae Gill 1892

Family Bembridae Kaup 1873 
Family Triglidae Rafinesque 1815

Family Peristediidae Jordan \& Gilbert 1883

Family Hoplichthyidae Kaup 1873

Family Platycephalidae Swainson 1839

Suborder Cottoidei

Infraorder Anoplopomatales

Family Anoplopomatidae Jordan \& Gilbert 1883

Infraorder Zoarcales

Family Zoarcidae Swainson 1839

Family Anarhichadidae Bonaparte 1835

Family Neozoarcidae Jordan \& Snyder 1902

Family Eulophiidae Smith 1902

Family Stichaeidae Gill 1864

Family Lumpenidae Jordan \& Evermann 1898

Family Opisthocentridae Jordan \& Evermann 1898

Family Pholidae Gill 1893

Family Ptilichthyidae Jordan \& Gilbert 1883

Family Zaproridae Jordan 1896

Family Cryptacanthodidae Gill 1861

Family Cebidichthyidae Gill 1862

Family Scytalinidae Jordan \& Starks 1895

Family Bathymasteridae Jordan \& Gilbert 1883

Infraorder Gasterosteales

Family Aulorhynchidae Gill 1861

Family Hypoptychidae Steindachner 1880

Family Gasterosteidae Bonaparte 1831

Infraorder Zaniolepidales

Family Zaniolepididae Jordan \& Gilbert 1883

Infraorder Hexagrammales

Family Hexagrammidae Jordan 1888

Infraorder Cottales

Family Trichodontidae Bleeker 1859

Family Cyclopteridae Bonaparte 1831

Family Liparidae Gill 1861 [ICZN Opinion 1673]

Family Jordaniidae Jordan \& Evermann 1898

Family Rhamphocottidae Jordan \& Gilbert 1883

Family Scorpaenichthyidae Jordan \& Evermann 1898

Family Agonidae Swainson 1839

Family Cottidae Bonaparte 1831

Family Psychrolutidae Günther 1861

\section{Subclass SARCOPTERYGII}

\section{Infraclass ACTINISTIA [Coelacanthida or Coelacanthimorpha]}

Family $\uparrow$ Miguashaiidae Forey 1998

$†$ Miguashaiidae Schultze 1993: 660 (family) †Miguashaia Schultze 1973 [name only, published after 1960, not available, Art. 13.1.1]

$†$ Miguashaiidae Cloutier \& Ahlberg in Stiassny, Parenti \& Johnson 1996: 451 (family) $\uparrow$ Miguashaia Schultze 1973 [name only, published after 1960, not available, Art. 13.1.1]

$\uparrow$ Miguashaiidae Forey 1998: 223 (family) †Miguashaia Schultze 1973 [family name sometimes seen as $\dagger$ Miguashaididae] 
Family $\uparrow$ Diplocercididae Berg 1940

$\uparrow$ Diplocercidae Berg 1940: 391 (family) †Diplocercides Stensiö 1922 [corrected to $\dagger$ Diplocercididae by Camp, Welles \& Green 1949: 271, confirmed by Fowler 1971b: 14; family name sometimes seen as $\uparrow$ Diploceriidae]

$\dagger$ Euporosteidae Romer 1966: 362 (family) †Euporosteus Jaekel 1927 [name only, published after 1960, not available, Art. 13.1.1]

Family $\uparrow$ Hadronectoridae Lund \& Lund 1984

$†$ Hadronectoridae Lund \& Lund 1984: 237 (family) †Hadronector Lund \& Lund 1984

Family $\uparrow$ Rhabdodermatidae Berg 1955, spelling in prevailing recent practice

$\dagger$ Rhabdodermidae Berg 1955: 100 (family) $†$ Rhabdoderma Reis 1888 [family name also seen as $\uparrow$ Rhabdodermatidae]

Family $\uparrow$ Sasseniidae Forey 1998

$†$ Sasseniidae Forey 1998: 299 (family) †Sassenia Stensiö 1921

Family $\dagger$ Laugiidae Berg 1940

$\dagger$ Laugiidae Berg 1940: 392 (family) †Laugia Stensiö 1932

$\uparrow$ Coccodermidae White 1956: 66 (family) †Coccoderma Zittel 1887

\section{Order Coelacanthiformes}

Suborder $\uparrow$ Coelacanthoidei

Family $\uparrow$ Whiteiidae Forey 1998

$\dagger$ Whiteiidae Maisey 1991: 312 (family) †Whiteia Moy-Thomas 1935 [name only, published after 1960, not available, Art. 13.1.1]

$\uparrow$ Whiteiidae Schultze 1993: 660 (family) †Whiteia Moy-Thomas 1935 [name only, published after 1960, not available, Art. 13.1.1]

†Whiteiidae Cloutier \& Ahlberg in Stiassny, Parenti \& Johnson 1996: 450 (family) †Whiteia Moy-Thomas 1935 [name only, published after 1960, not available, Art. 13.1.1]

$\dagger$ Whiteiidae Forey 1998: 225 (family) †Whiteia Moy-Thomas 1935

Family $\uparrow$ Rebellatricidae Wendruff \& Wilson 2012

$\uparrow$ Rebellatricidae Wendruff \& Wilson 2012: 500 (family) †Rebellatrix Wendruff \& Wilson 2012

Family †Coelacanthidae Agassiz 1844

†Célacanthes Agassiz 1844 Tome II pt. 2: 168 (family) †Coelacanthus Agassiz ?1839 [latinized to $†$ Caelacanthidae by Bonaparte 1846: 4; latinized to $†$ Coelacanthoides by Giebel 1846: 65, latinized to $\dagger$ Coelacanthi by $M^{\prime}$ Coy 1848: 2 , latinized to $†$ Coelacanthoidei by Bleeker 1859: XVII, latinized to †Coelacanthidae by Günther 1880: 365 ; considered valid with this authorship by Müller 1846: 151, by Bronn 1849: 654, by Quenstedt 1852: 228, by Morris 1856: 315, by Pander 1857: 42, by Andrews et al. 1967: 642, by Schultze et al. 1982: 46, by Maisey 1991: 303 and by Lambers 1996: 396 Art. 11.7.2]

\section{Suborder Latimerioidei}

Family $\uparrow$ Mawsoniidae Forey 1998

$\dagger$ Diplurinae Maisey 1991: 312 (subfamily) †Diplurus Newberry 1878 [family-group name preoccupied by Dipluridae Simon 1892 in spiders Arachnida, not to be used]

$\uparrow$ Mawsoniidae Schultze 1993: 660 (family) †Mawsonia Woodward 1907 [name only, published after 1960, not available, Art. 13.1.1]

$\uparrow$ Mawsoniidae Cloutier \& Ahlberg in Stiassny, Parenti \& Johnson 1996: 450 (family) $\uparrow$ Mawsonia Woodward 1907 [name only, published after 1960, not available, Art. 13.1.1]

$\uparrow$ Mawsoniidae Forey 1998: 300 (family) †Mawsonia Woodward 1907

Family Latimeriidae Berg 1940, name in prevailing recent practice

$\dagger$ Macropomidae Owen 1860: 144 (family) †Macropoma Agassiz 1835

$\dagger$ Undinidae Whitley 1940: 242 (family) †Undina Münster 1834 [type genus inferred from the stem; name only, used as valid before 2000?; not available] 


\section{Infraclass $\uparrow$ ONYCHODONTIDA}

Order $\uparrow$ Onychodontiformes

Family †Onychodontidae Woodward 1891

$\dagger$ Onychodontidae Woodward 1891a: 391 (family) †Onychodus Newberry 1857 [family name sometimes seen as $\dagger$ Onychodidae]

$\dagger$ Pycnacanthidae Lebedev 1995: 362 (family) †Pycnacanthus Fischer von Waldheim 1852

\section{Infraclass DIPNOMORPHA}

\section{Superorder $\uparrow$ Porolepidimorpha}

Order $\uparrow$ Porolepidiformes

Family $\uparrow$ Porolepididae Berg 1940, spelling in prevailing recent practice

$†$ Porolepidae Berg 1940: 390 (family) †Porolepis Woodward 1891 [family name also seen as $\dagger$ Porolepididae]

Family $\dagger$ Holoptychiidae Giebel 1847

$\dagger$ Holoptychii Giebel 1847: 270 (family) $\dagger$ Holoptychius Agassiz 1839 [changed to $\dagger$ Holoptychidae by Owen 1860: 156, corrected to †Holoptychiidae by Günther 1880: 365 ; correct stem is Holoptychi-]

$\dagger$ Dendrodonten Pander 1860: 24 (family) †Dendrodus Owen 1841 [published not in latinized form before 1900, not available, Art. 11.7.2; also seen as †Dendrodontés Eichwald 1860: 1557; the genus $\uparrow$ Dendrodus Owen 1841 is not recognized anymore because of uncertainty of typification; not $\uparrow$ Dendrodus Whiteaves 1897 in $\uparrow$ Protodontidae]

$\uparrow$ Glyptolépides Eichwald 1860: 1566 (family) †Glyptolepis Agassiz 1844 [published not in latinized form before 1900, not available, Art. 11.7.2]

$\dagger$ Dendrodontidae Traquair 1889: 490 (family) †Dendrodus Owen 1841 [as the type genus Dendrodus Owen 1841 is not recognized anymore because of uncertainty of typification, the family-group name is not to be used; not $\uparrow$ Dendrodus Whiteaves 1897 in $\uparrow$ Protodontidae]

Superorder Dipnoi [Ceratodontae]

Family †Powichthyidae Jessen 1980

$†$ Powichthyiidae [†Powichthyidae] Jessen 1980: 209 (family) †Powichthys Jessen 1975

Family †Youngolepididae Gardiner 1984

†Youngolepididae Gardiner 1984: 404 (family) †Youngolepis Chang \& Yu 1981 [family name sometimes seen as $\uparrow$ Youngolepidae]

\section{Order $\uparrow$ Diabolepidiformes}

Family $\uparrow$ Diabolepididae

$\uparrow$ Diabolepididae Schultze 1993: 657 (family) †Diabolepis Zhang \& Yu 1987 [name only, published after 1960, not available, Art. 13.1.1]

$\uparrow$ Diabolepididae Cloutier \& Ahlberg 1996: 451 (family) †Diabolepis Zhang \& Yu 1987 [name only, published after 1960, not available, Art. 13.1.1]

†Diabolepididae Nelson, Grande \& Wilson 2016: 107 (family) †Diabolepis Zhang \& Yu 1987 [not published according to the rules, not available]

\section{Order $\uparrow$ Dipnorhynchiformes}

Family †Uranolophidae Miles 1977

†Uranolophidae Miles 1977: 308 (family) †Uranolophus Denison 1968 [family name sometimes seen as $\uparrow$ Melanognathidae]

Family $\dagger$ Dipnorhynchidae Berg 1940

$\dagger$ Dipnorhynchidae Berg 1940: 385 (family) †Dipnorhynchus Jaekel 1927

$\dagger$ Speonesydrionidae Campbell \& Barwick 1990: 165 (family) †Speonesydrion Campbell \& Barwick 1983 [family name sometimes seen as $\uparrow$ Speonesydriidae] 


\section{Order $\uparrow$ Dipteriformes}

Family $\dagger$ Stomiahykidae Bernacsek 1977

$\dagger$ †Stomiahykidae Bernacsek 1977: 177 (family) †Stomiahykus Bernacsek 1977

Family $†$ Dipteridae Agassiz 1844

†Diptériens Agassiz 1844 Tome II pt. 1: 308 (family) †Dipterus Sedgwick \& Murchison 1828 [latinized to $†$ Dipteri by Eichwald 1846: 307; latinized to $\uparrow$ Dipteridae by Owen 1846: 50, latinized to $\uparrow$ Dipterida by Vogt 1851: 131, latinized to $†$ Dipterini by Pander 1856: 79 and Pander 1858: 5; considered valid with this authorship by Müller 1846: 151, by Bronn 1849: 655, by Pander 1856: 79 and by Quenstedt 1885: 349 Art. 11.7.2]

$\dagger$ Ctenododipteridae Günther 1880: 359 (family) ? †Dipterus Sedgwick \& Murchison 1828 [no stem of the type genus, not available, Art. 11.7.1.1]

$\dagger$ Rhinodipteridae Campbell \& Barwick 1990: 165 (family) $†$ Rhinodipterus Gross 1956

†Orlovichthyinae Krupina 2004: 395 (subfamily) †Orlovichthys Krupina 1980

Family $\uparrow$ Chirodipteridae Campbell \& Barwick 1990

$\uparrow$ Chirodipteridae Campbell \& Barwick 1990: 165 (family) †Chirodipterus Gross 1933

$\dagger$ Pillararhynchinae Long 1992: 12 (subfamily) †Pillararhynchus Campbell \& Barwick 1990

$\dagger$ Conchodontinae Krupina 2004: 398 (subfamily) †Conchodus M'Coy 1848

Family $\uparrow$ Rhynchodipteridae Moy-Thomas 1939

$†$ Rhynchodipteridae Moy-Thomas 1939: 100 (family) $†$ Rhynchodipterus Säve-Söderbergh 1937

Family $\dagger$ Holodipteridae

$\dagger$ Holodontidae Gorizdro-Kulczycka 1950: 54 (family) †Holodus Pander 1858 [type genus preoccupied by $\uparrow$ Holodus Agassiz 1845 in fishes; invalid, Art. 39]

$\dagger$ Holodipterida Moy-Thomas \& Miles 1971: 141 (order) $\uparrow$ Holodipterus White \& Moy-Thomas 1940 [no family-group name; also name only]

$\dagger$ tholodipterids Moy-Thomas \& Miles 1971: 149 (family?) $†$ Holodipterus White \& Moy-Thomas 1940 [published not in latinized form after 1899, not available]

$\dagger$ †holodipterids Miles 1977: 241, 307 (family?) †Holodipterus White \& Moy-Thomas 1940 [published not in latinized form after 1899, not available]

Family $\uparrow$ Phaneropleuridae Huxley 1861

$\uparrow$ Phaneropleurini Huxley 1861: 24 (family) $†$ Phaneropleuron Huxley 1859

$\dagger$ Scaumenaciidae Berg 1940: 385 (family) $†$ Scaumenacia Traquair 1893 [family name sometimes seen as $†$ Scaumenacidae]

Family $\uparrow$ Fleurantiidae Moy-Thomas 1939

$†$ Fleurantidae Moy-Thomas 1939: 100 (family) †Fleurantia Graham-Smith \& Westoll 1937 [stem corrected to Fleuranti- by Berg 1940: 385, confirmed by Fowler 1971a: 210]

\section{Order $\uparrow$ Ctenodontiformes}

Family $†$ Sagenodontidae Jaekel 1911

$\uparrow$ Sagenodontidae Jaekel 1911: 78 (family) $†$ Sagenodus Owen 1867

Family $\dagger$ Ctenodontidae Traquair 1890

$\uparrow$ Ctenodipteri Pander 1856: 79 (family) $\uparrow$ Ctenodus Agassiz 1838 [also seen as $†$ Ctenodipterini

Pander 1857: 1; no stem of the type genus, not available, Art. 11.7.1.1]

$†$ Cténodiptériens Eichwald 1860: 1534 (family) †Ctenodus Agassiz 1838 [published not in latinized form before 1900, not available, Art. 11.7.2]

$\dagger$ Ctenododipteridae Günther 1880: 359 (family) ? †Ctenodus Agassiz 1838 [no stem of the type genus, not available, Art. 11.7.1.1]

$\dagger$ Ctenodontidae Traquair 1890: 15 (family) $\dagger$ Ctenodus Agassiz 1838 [family name sometimes seen as $\uparrow$ Ctenodidae; not $\uparrow$ Ctenodontidae Wohrmann 1893 in Mollusca]

Family $\uparrow$ Conchopomatidae Berg 1940, spelling in prevailing recent practice

†Conchopomidae Berg 1940: 386 (family) †Conchopoma Kner 1868 [family name also seen as $\dagger$ Conchopomatidae] 
Family †Ganopristodidae Fowler 1958

$\dagger$ Uronemidae Traquair 1891: 387 (family) †Uronemus Agassiz 1844 (type genus preoccupied by Uronemus Rafinesque 1815 in Vermes; invalid, Art. 39]

$\dagger$ Ganopristodidae Fowler 1958: 3 (family) †Ganopristodus Traquair 1881

\section{Order Ceratodontiformes}

Family Lepidosirenidae Bonaparte 1841

Family Protopteridae Peters 1855

Family $\uparrow$ Gnathorhizidae Olson 1951

$\uparrow$ Gnathorhizidae Olson 1951: 180 (family) †Gnathorhiza Cope 1883 [name only, but used as valid by Miles 1977: 308 and by Smith 1979: 32 Art. 13.2.1]

Family Neoceratodontidae Schultz 1948

Family †Ceratodontidae Günther 1871

$\uparrow$ Ceratodontina Günther 1871: 554 (subfamily) †Ceratodus Agassiz 1838 [family name sometimes seen as $\uparrow$ Ceratodidae]

$\uparrow$ Protoceratodontidae Miles 1977: 308 (family) †Ceratodus sturii [no valid type genus, not available, Art. 11.7.1.1]

$\uparrow$ Arganodontidae Martin 1982a: 414 (family) $\uparrow$ Arganodus [also in Martin 1982b]

$\uparrow$ Asiatoceratodontidae Castro, Toledo, de Sousa \& Medeiros 2004: 264 (family) $\uparrow$ Asiatoceratodus Vorobyeva 1967 [not published according to the rules, not available; the name $\uparrow$ Asiatoceratodontidae is, contrary to numerous citations, not mentioned in Vorobyeva 1967]

$\uparrow$ Asiatoceratodontidae Agnolin 2010: 189 (family) †Asiatoceratodus Vorobyeva 1967 [not published according to the rules, not available]

$\uparrow$ Asiatoceratodontidae Nelson, Grande \& Wilson 2016: 109 (family) $\uparrow$ Asiatoceratodus Vorobyeva 1967 [not published according to the rules, not available]

Family $\uparrow$ Ptychoceratodontidae Martin 1982

$\nmid$ Ptychoceratodontidae Martin 1982a: 414 (family) †Ptychoceratodus Jaekel 1926 [also in Martin 1982b]

\section{Infraclass $\uparrow$ RHIZODONTIDA}

\section{Order $\uparrow$ Rhizodontiformes}

Family $\uparrow$ Rhizodontidae Traquair 1881

$†$ Rhizodontidae Traquair 1881a: 18 (family) †Rhizodus Owen 1840

\section{Infraclass $\uparrow$ OSTEOLEPIDIDA}

\section{Order $\uparrow$ Osteolepidiformes}

Incertae sedis:

$\uparrow$ Diplopteroidei Bleeker 1859: XVII (family) †Diplopterus Agassiz 1835 [type genus preoccupied by Diplopterus Boie 1826 in Aves; invalid, Art. 39]

†Glyptolaemini Traquair 1888b: 515 (subfamily) †Glyptolaemus Huxley 1859 [type genus inferred from the stem, Art. 11.7.1.1]

$\dagger$ Lamprotolepididae Vorobyeva 1975: 54 (family) †Lamprotolepis Vorobyeva 1977 [no valid type genus, not available, Art. 11.7.1.1]

$\dagger$ Lamprotolepididae Young, Long \& Ritchie 1992: 61 (family) †Lamprotolepis Vorobyeva 1977 [name only, published after 1960, not available, Art. 13.1.1]

$\uparrow$ Megistolepidinae Vorobyeva 1975: 54 (subfamily) $\uparrow$ Megistolepis Obruchev 1955 [name only, published after 1960, not available, Art. 13.1.1]

$\dagger$ Pleiopteri Fitzinger 1873: 52 (family) $\uparrow$ Pleiopterus Agassiz 1835 [also as a new family, $\uparrow$ Pleiopteridae, in Whitley 1951: 67] 
$\uparrow$ Rhizodopsidae Berg 1940: 389 (family) $\uparrow$ Rhizodopsis Young 1866 [sometimes seen as $\uparrow$ Rhizodopidae or $\uparrow$ Rhizodopsididae]

$\dagger$ Rhombodipteridae Lütken 1871: 334 (family) ? [no stem of the type genus, not available, Art. 11.7.1.1]

$\dagger$ Saurodipteridae M’Coy 1855: 585 (family) ? †Diploterax M'Coy 1855 [no stem of the type genus, not available, Art. 11.7.1.1]

†Saurodipteridae Günther 1880: 365 (family) ? [no stem of the type genus, not available, Art. 11.7.1.1]

$\uparrow$ Thysanolepidinae Vorobyeva 1975: 54 (subfamily) †Thysanolepis Vorobyeva 1977 [no valid type genus, not available, Art. 11.7.1.1]

$\uparrow$ Thysanolepididae Young, Long \& Ritchie 1992: 61 (family) †Thysanolepis Vorobyeva 1977 [name only, published after 1960, not available, Art. 13.1.1]

$\dagger$ †ilulichthyinae Vorobyeva 1977a: 165 (subfamily) †Vilulichthys Vorobyeva 1977

\section{Suborder $\uparrow$ Osteolepidoidei}

Family $\uparrow$ Thursiidae Borgen \& Nakrem 2016

$\dagger$ Thursiidae Borgen \& Nakrem 2016: 383 (family) $†$ Thursius Traquair 1888

Family $\uparrow$ Osteolepididae Cope 1887, spelling in prevailing recent practice

$\dagger$ Osteolepididae Cope 1887: 1018 (family) $†$ Osteolepis Valenciennes 1829 [type genus inferred from the stem, Art. 11.7.1.1; family name also seen as †Osteolepidae]

$\uparrow$ Glyptopomidae Goodrich 1909: 285 (family) †Glyptopomus Agassiz 1844

Family $\uparrow$ Megalichthyidae Hay 1902

$\dagger$ Megalichthyidae Hay 1902: 359 (family) $†$ Megalichthys Agassiz 1835

$\dagger$ Ectosteorhachidae Berg 1940: 389 (family) $†$ Ectosteorhachis Cope 1880 [also as a new subfamily, †Ectosteorhachinae, in Borgen \& Nakrem 2016: 412]

$\uparrow$ Parabatrachidae Berg 1955: 92 (family) $†$ Parabatrachus Owen 1853

$\uparrow$ Askerichthyinae Borgen \& Nakrem 2016: 419 (subfamily) †Askerichthys Borgen \& Nakrem 2016

\section{Suborder $\uparrow$ Cyclolepidoidei}

†Eopodoidea Borgen \& Nakrem 2016: 420 (superfamily) ? [no stem of the type genus, not available, Art. 11.7.1.1]

$\dagger$ Parapodoidea Borgen \& Nakrem 2016: 463 (superfamily) ? [no stem of the type genus, not available, Art. 11.7.1.1]

Family $\uparrow$ Gyroptychiidae Berg 1955

$\uparrow$ Gyroptychiidae Berg 1955: 90 (family) †Gyroptychius M’Coy 1848

Family $\uparrow$ Panderichthyidae Vorobyeva 1968, name in prevailing recent practice

$\dagger$ Elpistostegidae Romer 1945: 590 (family) †Elpistostege Westoll 1938 [name only, but used as valid by Romer 1947: 311, by Romer 1966: 362 and by Cloutier \& Ahlberg in Stiassny, Parenti \& Johnson 1996: 460 Art. 13.2.1; also as a new subfamily, †Elpistosteginae, in Borgen \& Nakrem 2016: 335]

$\dagger$ Panderichthyidae Vorobyeva in Vorobyeva \& Lyarskaya 1968: 74 (family) $†$ Panderichthys Gross 1941 [Borgen \& Nakrem 2016: 3 use the name $\uparrow$ Panderichthyidae for the family, because "the name is in common use and is more informative". Furthermore, the genus $\dagger$ Elpistostege could be a tetrapod.]

Family $\uparrow$ Chrysolepididae Borgen \& Nakrem 2016

†Chrysolepididae Borgen \& Nakrem 2016: 438 (family) †Chrysolepis Lebedev 1983

Family $\uparrow$ Tristichopteridae Cope 1887

$\dagger$ Tristichopteridae Cope 1887: 1018 (family) $\dagger$ Tristichopterus Egerton 1861 [type genus inferred from the stem, Art. 11.7.1.1] 
$\dagger$ Eustenopteridae Berg 1955: 94 (family) †Eustenopteron Whiteaves 1881 [Borgen \& Nakrem 2016: 4 prefer the family name $\dagger$ Eustenopteridae being 'more convenient' and by referring to the first (!) edition of the Code]

$\uparrow$ Platycephalichthyinae Vorobyeva 1975: 54 (subfamily) †Platycephalichthys Vorobyeva 1959

$\uparrow$ Notorhizodontidae Davis 1994: 70 (family) †Notorhizodon Young, Long \& Ritchie 1992 [name only, published after 1960, not available, Art. 13.1.1]

$\uparrow$ Mandageriinae Young 2008: 321 (subfamily) $\uparrow$ Mandageria Johanson \& Ahlberg 1997

Family $\uparrow$ Medoeviidae Borgen \& Nakrem 2016

$\dagger$ Medoevididae [†Medoeviidae] Borgen \& Nakrem 2016: 463 (family) †Medoevia Lebedev 1995 [although family-group names proposed after 1999 cannot be corrected (Arts 29.3.3 and

29.4), I think a simple typing error can explain the incorrectly spelled family-group name; the correct stem is deemed to be Medoevi-]

Family $\uparrow$ Canowindridae Young, Long \& Ritchie 1992

$†$ Canowindridae Young, Long \& Ritchie 1992: 9 (family) †Canowindra Thomson 1973

\section{Acknowledgements}

I would like to thank the following persons for their help and information: Plamen Andreev, Alexandre F. Bannikov, William Bemis, Philippe Bouchet, Martin Ebert, Bill Eschmeyer, John Finarelli, Ron Fricke, Guillaume Guinot, Kishor Kumar, Paul van der Laan, Tiiu Märss, Alla Minikh, Ken Monsch, HansPeter Schultze, Andrea Tintori, Susan Turner, Mark Wilson and Elsbeth Zwart.

Special thanks to the staff of the libraries of the Naturalis Biodiversity Center, Leiden and Denieuwebibliotheek, Almere for their great help in obtaining numerous references. I am also obliged to all contributors to the Biodiversity Heritage Library in making the task to check the literature a lot easier. I am grateful to the reviewers for their helpful suggestions to improve the manuscript.

Finally, I remain very grateful to my wife Hana for inspiring me and helping me to work on the family names of fossil fishes. Sadly, she passed away before I could finish the manuscript, but I think she would be pleased with the result.

\section{References}

Abel O. 1919. Die Stämme der Wirbeltiere. Walter de Gruyter \& Co, Berlin. https://doi.org/10.5962/bhl.title.2114

Afanassieva O.B. 1991. [The Osteostracans of the USSR (Agnatha)]. Transactions of the Palaeontological Institute (Academy of Sciences of the USSR) / Trudy Paleontologicheskogo Instituta, Akademia Nauk SSSR 248: 1-144. [In Russian, with English summary and contents. Author also seen as Afanas'eva.]

Afanassieva O.B. 1996. On the morphology and systematic position of tremataspid osteostracan Aestiaspis viitaensis (Agnatha). Paleontological Journal 30 (5): 566-570. [Also in Russian Paleontologicheskii Zhurnal 1996 (4): 68-72.]

Afanassieva O.B. 2004. [Agnathans and early fishes: subclass Osteostraci (osteostracans)]. In: Novitskaya L.I. \& Afanassieva O.B. (eds) Iskopayemyye pozvonochnyye Rossii i sopredel'nykh stran. Beschelyustnyye i drevniye ryby. Spravochnik dlya paleontologov, biologov i geologov [Fossil Vertebrates of Russia and adjacent countries. The reference book for paleontologists, biologists and geologists]: 210-267. GEOS, Moscow. [In Russian.]

Agassiz L. 1832. Untersuchungen über die fossilen Fische aus der Lias-Formation. Jahrbuch für Mineralogie, Geognosie, Geologie und Petrefaktenkunde 3: 139-149. 
Agassiz L. 1833-43. Recherches sur les poissons fossiles. Neuchâtel, Switzerland, 5 vols with atlas. [Dates as reported by Brown in Woodward \& Sherborn (1890: xxv-xxix) with some correction by Sherborn (1922: xv).] https://doi.org/10.5962/bhl.title.4275

Agnolin F. 2010. A new species of the genus Atlantoceratodus (Dipnoiformes: Ceratodontoidei) from the Uppermost Cretaceous of Patagonia and a brief overview of fossil dipnoans from the Cretaceous and Paleogene of South America. Brazilian Geographical Journal: Geosciences and Humanities Research Medium 1 (2): 162-210. Available from https://dialnet.unirioja.es/descarga/articulo/3638695.pdf [accessed 27 Jul. 2018].

Aldinger H. 1937. Permische Ganoidfische aus Ostgrönland. Meddelelser om Grønland 102 (3): 1-392.

Altner M. \& Reichenbacher B. 2015. †Kenyaichthyidae fam. nov. and †Kenyaichthys gen. nov. First record of a fossil aplocheiloid killifish (Teleostei, Cyprinodontiformes). PLoS One 10 (4): e0123056. https://doi.org/10.1371/journal.pone.0123056

Alvarado-Ortega J. 2004. Description and relationships of a new ichthyodectiform fish from the Tlayúa Formation (Early Cretaceous: Albian), Puebla, Mexico. Journal of Vertebrate Paleontology 24 (4): 802813. https://doi.org/10.1671/0272-4634(2004)024[0802:DAROAN]2.0.CO;2

Andersson E. [= Stensiö E.] 1916. Über einige Trias-Fische aus der Cava Trefontane, Tessin. Bulletin of the Geological Institution of the University of Uppsala 15: 13-33.

Andreev P.S., Coates M.I., Shelton R.M., Cooper P.R., Smith M.P. \& Sansom I.J. 2015. Upper Ordovician chondrichthyan-like scales from North America. Palaeontology 58 (4): 691-704. https://doi.org/10.1111/pala.12167

Andreev P.S., Coates M.I., Karatajūtè-Talimaa V., Shelton R.M., Cooper P.R. \& Sansom I.J. 2017. Elegestolepis and its kin, the earliest monodontode chondrichthyans. Journal of Vertebrate Paleontology 37 (1): e1245664. https://doi.org/10.1080/02724634.2017.1245664

Andrews S.M., Gardiner B.G., Miles R.S. \& Patterson C. 1967. Chapter 26 Pisces. In: Harland W.B. et al. (eds) The Fossil Record: 637-683. Geological Society of London, London.

Applegate S.P. 1970. The vertebrate fauna of the Selma Formation of Alabama, Part VIII The fishes. Fieldiana Geology Memoires 3 (8): 381-433. Available from https://www.biodiversitylibrary.org/item/25157 [accessed 27 Jul. 2018].

Applegate S.P. 2001a. The Origin of the Lamniform Sharks, a Study in Morphology and Paleontology of Recent and Fossil Genera. Abstracts of the Annual Meeting of the American Elasmobranch Society 2001. [Work not published according to ICZN Art. 8.]

Applegate S.P. 2001b. The Pycnodont Fauna from the lower Cretaceous of Tepexi de Rodriguez (Puebla, Mexico): 3. Abstracts of the International Meeting on Mesozoic Fishes Systematics, Paleoenvironments and Biodiversity. Serpiano-Monte San Giorgio (TI-CH) 26-31 August 2001. [Work not published according to ICZN Art. 8.]

Arambourg E. 1927. Les poissons fossiles d'Oran, matériaux pour la carte géologique de l'Algérie (1 ${ }^{e}$ série Paléontologie no. 6). J. Carbonel, Algiers.

Arambourg C. 1941. Le groupe des Ganopristinés. Bulletin de la Société géologique de France (série 5) 10: $127-147$.

Arambourg C. 1944. Note préliminaire sur quelques poissons fossiles nouveaux. I. Les poissons du Jebel Tselfat (Maroc). Bulletin de la Société géologique de France (série 5) 13: 281-288.

Arambourg C. 1955. Les poissons crétacés du Jebel Tselfat (Maroc). Notes et Mémoires du Service géologique du Maroc No. 118: 1-188. 
Arambourg C. 1958. Classe des Placodermes (Placodermi). In: Grassé P.P. (ed.) Traité de Zoologie Tome 13 Fascicule 3: 1990-2009. Masson \& Cie, Paris.

Arambourg C. 1967. Résultats scientifiques de la mission C. Arambourg en Syrie et en Iran (19381939). II. Les poissons oligocènes de l'Iran. Notes et Mémoires sur le Moyen-Orient 8: 11-247.

Arambourg C. \& Bertin L. 1958. Super-ordres des holostéens et des halecostomes (Holostei et Halecostomi). In: Grassé P.P. (ed.) Traité de Zoologie Tome 13 Fascicule 3: 2173-2203. Masson \& Cie, Paris.

Arratia G. 1981. Varasichthys ariasi n. gen. et sp. from the Upper Jurassic of Chile (Pisces, Teleostei, Varasichthyidae n. fam.). Palaeontographica (Abteilung A: Paläozoologie-Stratigraphie) 175 (4/6): $107-139$.

Arratia G. 1982. Chongichthys dentatus, new genus and species from the Late Jurassic of Chile (Pisces, Teleostei: Chongichthyidae, new family). Journal of Vertebrate Paleontology 2 (2): 133-149. Available from http://www.jstor.org/stable/4522889 [accessed 27 Jul. 2018].

Arratia G. 1997. Basal teleosts and teleostean phylogeny. Palaeo Ichthyologica 7: 1-168.

Arratia G. 2016. New remarkable Late Jurassic teleosts from southern Germany: Ascalaboidae n. fam., its content, morphology, and phylogenetic relationships. Fossil Record 19: 31-59. https://doi.org/10.5194/fr-19-31-2016

Arratia G. 2017. New Triassic teleosts (Actinopterygii, Teleosteomorpha) from northern Italy and their phylogenetic relationships among the most basal teleosts. Journal of Vertebrate Paleontology 37 (2): [1-24] e1312690. https://doi.org/10.1080/02724634.2017.1312690

Arratia G. \& Chorn J. 1998. A new primitive acanthomorph fish from the Greenhorn Formation (Late Cretaceous) of Nebraska. Journal of Vertebrate Paleontology 18 (2): 301-314. Available from http://www.jstor.org/stable/4523900 [accessed 27 Jul. 2018].

Arratia G. \& Schultze H.-P. 2015. A new fossil actinistian from the Early Jurassic of Chile and its bearing on the phylogeny of Actinistia. Journal of Vertebrate Paleontology 35 (5): e983524. https://doi.org/10.1080/02724634.2015.983524

Arsenault M. \& Janvier Ph. 1995. Combien d'Ostéostracés à Miguasha? In: Lelièvre H., Wenz S. \& Cloutier R. (eds) Premiers Vertébrés et Vertébrés inférieurs. Geobios Mémoire Spécial 19: 19-22.

Asmuss H. 1856. Das vollkommenste Hautskelett der bisher bekannten Thierreihe, an fossilen Fischen des alten rothen Sandsteins aufgefunden und aus ihren Resten erläutert. Abhandlung zur Erlangung der Magisterwürde. Schünmanns Wittwe \& Mattiesen, Dorpat.

Azpelicueta M.M. \& Cione A.L. 2011. Redescription of the Eocene catfish Bachmannia chubutensis (Teleostei: Bachmanniidae) of southern South America. Journal of Vertebrate Paleontology 31 (2): 258-269. https://doi.org/10.1080/02724634.2011.550351

Bannikov A.F. 1984. The new subfamily of carangid fishes. Miscellanea Paleontologica n. 2. Studi e ricerche sui giacimenti terziari di Bolca IV: 319-321.

Bannikov A.F. 1990. Iskopayemye stavridovye i voron-ryby SSSR [Fossil carangids and apolectids of the USSR]. Trudy Paleontologicheskogo Instituta (Akademiya Nauk SSSR) [Transactions of the Paleontological Institute of the Academy of Sciences of the USSR] 244: 1-108. [In Russian.]

Bannikov A.F. 1991a. On the systematic position of the family Caproidae with reference to the Eocene genus Acanthonemus. Voprosy ikhtiologii 31 (2): 179-188. [In Russian; English translation in Journal of Ichthyology: 47-58.] 
Bannikov A.F. 1991b. A new family of the Oligocene perciform fishes. Paleontologicheskii Zhurnal 1991 (4): 88-94.

Bannikov A.F. 1991c. An Eocene veliferoid (Teleostei, Lampridiformes) from Bolca. Miscellanea Paleontologica n. 3. Studi e ricerche sui giacimenti terziari di Bolca VI (for 1990): 161-174.

Bannikov A.F. 1995. Morphology and phylogeny of fossil stromateoid fishes (Perciformes). Geobios 28 (Supplement 2): 177-181. https://doi.org/10.1016/S0016-6995(95)80109-X

Bannikov A.F. 1999. Unusual new percoid fish from the Eocene of Bolca. Miscellanea Paleontologica n. 5. Studi e ricerche sui giacimenti terziari di Bolca VIII: 117-128.

Bannikov A.F. 2004. Eocottidae, a new family of perciform fishes (Teleostei) from the Eocene of northern Italy (Bolca). Miscellanea Paleontologica n. 7. Studi e ricerche sui giacimenti terziari di Bolca $\mathrm{X}: 17-35$.

Bannikov A.F. 2005. New cardinalfishes (Perciformes, Apogonidae) from the Eocene of Bolca, northern Italy. Miscellanea Paleontologica n. 8. Studi e ricerche sui giacimenti terziari di Bolca XI: 119-140.

Bannikov A.F. 2006. Bassanichthys, a new replacement generic name for the Eocene fish Bassania Bannikov, 2004 (Teleostei, Perciformes). Paleontological Journal 40 (3): 340. https://doi.org/10.1134/S0031030106030166

Bannikov A.F. 2008. Revision of the atheriniform fish genera Rhamphognathus Agassiz and Mesogaster Agassiz (Teleostei) from the Eocene of Bolca, northern Italy. Miscellanea Paleontologica n. 9. Studi e ricerche sui giacimenti terziari di Bolca XII: 77-97. Available from http://museodistorianaturale.comune.verona.it/media/_Musei/_StoriaNaturale/_Allegati/83337_Bolca _p_77-98.pdf [accessed 27 Jul. 2018].

Bannikov A.F. 2011. A new percoid fish (Perciformes) related to Hendrixella from the Eocene of Bolca, Italy. Miscellanea Paleontologica n. 10. Studi e ricerche sui giacimenti terziari di Bolca XIII: 7-16. Available from http://museodistorianaturale.comune.verona.it/media/_Musei/_StoriaNaturale/_Allegati/ 01.Bannikov_007-016.indd.pdf [accessed 27 Jul. 2018].

Bannikov A.F. 2013. A new late neogene genus of croakers (Perciformes, Sciaenidae) from the Eastern Black Sea Region. Paleontological Journal 47 (2): 190-198. [Also in Russian Paleontologicheskii Zhurnal 47 (2): 60-69.] https://doi.org/10.1134/S0031030113020032

Bannikov A.F. 2014a. The new genus Eoleiognathus for the percoid fish Pygaeus dorsalis Agassiz from the Eocene of Bolca in northern Italy, a putative ponyfish (Perciformes, Leiognathidae). Miscellanea Paleontologica n. 12. Studi e ricerche sui giacimenti terziari di Bolca XV: 35-42. Available from http://museodistorianaturale.comune.verona.it/media/_Musei/_StoriaNaturale/_Allegati/Biblioteca/Studi\% 20Bolca/Vol.\%2015/04_Bannikov_35_42.pdf [accessed 27 Jul. 2018].

Bannikov A.F. 2014b. A new genus of the family Palaeocentrotidae (Teleostei, Lampridiformes) from the Oligocene of the Northern Caucasus and comments on other fossil Veliferoidei. Paleontological Journal 48 (6): 624-632. [Also in Russian Paleontologicheskii Zhurnal 48 (6): 50-58.] https://doi.org/10.1134/S0031030114060021

Bannikov A.F. \& Bacchia F. 2000. A remarkable clupeomorph fish (Pisces, Teleostei) from a new Upper Cretaceous marine locality in Lebanon. Senckenbergiana lethaea 80 (1): 3-11.

Bannikov A.F. \& Carnevale G. 2012. A long-bodied centriscoid fish from the basal Eocene of KabardinoBalkaria, northern Caucasus, Russia. Naturwissenschaften 99 (5): 379-389.

https://doi.org/10.1007/s00114-012-0912-6

Bannikov A.F. \& Carnevale G. 2017. Eocene ghost pipefishes (Teleostei, Solenostomidae) from Monte Bolca, Italy. Bollettino della Società Paleontologica Italiana 56 (3): 319-331. 
Bannikov A.F. \& Daniltshenko P.G. 1985. A new family of cenozoic bream (order Beryciformes). Transactions of the USSR Academy of Sciences (Earth science) 283: 197-199. [Second author also seen as Danil'chenco.]

Bannikov A.F. \& Fedotov V.F. 1984. Novoye semeystvo iskopayemykh kolyuchepyorykh ryb [A new family of fossil acanthopterygian fishes]. Doklady Akademii Nauk SSSR / Comptes rendus de l'Académie des sciences de l'URSS 276 (3): 708-710. [In Russian, with English summary, English edition: 178180.]

Bannikov A.F. \& Sorbini C. 2005. A new beryciform genus and family from the Cenomanian of Hajula (Lebanon). In: Extended abstracts of the fourth international meeting on Mesozoic fishes - systematics, homology, and nomenclature, August $8^{\text {th }}-14^{\text {th }} 2005$, Madrid, Spain, UAM Ediciones: 29-32. [Available publication?]

Bannikov A.F. \& Tyler J.C. 2002. A new genus and species of rabbitfish (Acanthuroidei: Siganidae) from the Eocene of Monte Bolca, Italy. Miscellanea Paleontologica n. 6. Studi e ricerche sui giacimenti terziari di Bolca IX: 37-45.

Bannikov A.F., Parin N.V. \& Pinna J. 1985. Rhamphexocoetus volans, gen. et sp. nov. a new beloniform fish (Beloniformes, Exocoetoidei) from the Lower Eocene of Italy. Voprosy Ikhtiologii 25 (2): 343-346. [In Russian, translated in Journal of Ichthyology: 150-155.]

Bannikov A.F., Carnevale G. \& Parin N.V. 2011. The new family Caucasichthyidae (Pisces, Perciformes) from the Eocene of the North Caucasus. Paleontological Journal 45 (1): 83-89. https://doi.org/10.1134/S0031030111010047

Bannikov A.F., Tyler J.C., Arcila D. \& Carnevale G. 2016. A new family of gymnodont fish (Tetraodontiformes) from the earliest Eocene of the Peri-Tethys (Kabardino-Balkaria, northern Caucasus, Russia). Journal of Systematic Palaeontology 15 (2): 129-146. [Published online 3 Mar. 2016: 1-18.] https://doi.org/10.1080/14772019.2016.1149115

Bannikov A.F., Carnevale G. \& Popov Y.A. 2017. An extraordinary pipefish (Teleostei, Syngnathidae) with fully developed anal fin from the Oligocene of the North Caucasus (SW Russia). Bollettino della Società Paleontologica Italiana 56 (1): 79-88.

Bardack D. \& Zangerl R. 1971. Lampreys in the fossil record. In: Hardisty M.W. \& Potter I.C. (eds) The Biology of Lampreys Volume 1: 67-84. Academic Press, London.

Bartholomai A. 2010. A new Albian teleost, Euroka dunravenensis gen. et sp. nov. and a new family, Eurokidae, from the Eromanga Basin of Queensland. Memoirs of the Queensland Museum-Nature 55 (1): 69-85. Available from https://biodiversitylibrary.org/page/54367009 [accessed 9 Sep. 2018].

Bartram A.W.H. 1975. The holostean fish genus Ophiopsis Agassiz. Zoological Journal of the Linnean Society 56 (3): 183-205. https://doi.org/10.1111/j.1096-3642.1975.tb00263.x

Bartram A.W.H. 1977. The Macrosemiidae, a Mesozoic family of holostean fishes. Bulletin of the British Museum (Natural History), Geology 29 (2): 137-234.

Available from https://biodiversitylibrary.org/page/36620553 [accessed 20 Sep. 2018].

Bassani Fr. 1879a. Vorläufige Mittheilungen über die Fischfauna der Insel Lesina. Verhandlungen der Kaiserlich Königlichen Geologischen Reichsanstalt 1879 (8): 162-170.

Available from http://www.zobodat.at/pdf/VerhGeolBundesanstalt_1879_0162-0170.pdf [accessed 20 Sep. 2018].

Bassani Fr. 1879b. Ueber einige fossile Fische von Comen. Verhandlungen der Kaiserlich Königlichen Geologischen Reichsanstalt 1879 (9): 204-205. 
Available from http://www.zobodat.at/pdf/VerhGeolBundesanstalt_1879_0204-0205.pdf [accessed 20 Sep. 2018].

Beaumont G. de 1960. Contribution à l'étude des genres Orthacodus Woodward et Notidanus Cuvier (Selachii). Mémoire suisse de Paléontologie 77: 4-36.

Beltan L. 1990. New Permian actinopterygian families from Uruguay. Acta Musei Reginaehradecensis series A Scientiae naturales 22 [for 1989]: 79-86.

Bemis W.E. 2016. Species and the fossil record of Fishes. In: Allmon W.D. \& Yacobucci M.M. (eds) Species and Speciation in the Fossil Record: 312-339. University of Chicago Press, Chicago.

Bemis K.E., Tyler J.C., Bemis W.E., Kumar K., Singh Rana R. \& Smith T. 2018. A gymnodont fish jaw with remarkable molariform teeth from the early Eocene of Gujarat, India (Teleostei, Tetraodontiformes). Journal of Vertebrate Paleontology 37 (6): [1-10] e1369422.

https://doi.org/10.1080/02724634.2017.1369422

Benton M.J. (ed.) 1993. The Fossil Record 2. Chapman \& Hall, London.

Berg L.S. 1936. Teleopterina n. g., a highly organized acanthopterygian from the Carboniferous of North America. Doklady Akademii Nauk SSSR / Comptes rendus de l'Académie des sciences de l'URSS (N.S.) 4 (7): 345-347.

Berg L.S. 1940. Sistema ryboobraznykh i ryb, nyne zhivushchikh i iskopaemykh / Classification of fishes, both recent and fossil. Trudy Zoologicheskogo instituta Akademiia nauk Soiuza Sovetskikh Sotsialisticheskikh Respublik / Travaux de l'Institut Zoologique de l'Académie des Sciences de l'URSS 5 (2): 87-517. [In Russian, with complete English translation.] [Published after 23 July.]

Berg L.S. 1941. Lower Triassic fishes of the Tunguska coal basin, Yenisei, Siberia [Fische aus der Untertrias der Tunguska-Niederung]. Bulletin of the Academy of Sciences USSR, Biological Sciences (3): 458-474. [In Russian.]

Berg L.S. 1955. Sistema ryboobraznykh i ryb, nyne zhivushchikh i isokopaemykh. 2-e izdanie [Classification of fishes and fish-like animals, living and fossil, $2^{\text {nd }}$ edition]. Trudy Zoologicheskogo Instituta Akademiia nauk Soiuza Sovetskikh Sotsialisticheskikh Respublik / Travaux de l'Institut zoologique de l'Académie des Sciences de l'URSS] 20: 1-286. [In Russian, German translation 1958: System der rezenten and fossilen Fischartigen und Fische. Deutscher Verlag der Wissenschaften, Berlin; citation to the 'German page numbers'.]

Berg L.S., Kazantseva A.A. \& Obruchev D.V. 1964. [Palaeonisci]. In: Obruchev D.V. (ed.) Osnovy paleontologii [Fundamentals of Paleontology] Vol. XI Agnatha, Pisces: 336-370. Izdatel'stvo 'Nauka', Moscow. [In Russian, English translation 1967, Fundamentals of Paleontology, Vol. XI. Agnatha, Pisces. Israel Program for Scientific Translations, Jerusalem.]

Bernacsek G.M. 1977. A lungfish cranium from the Middle Devonian of the Yukon Territory, Canada. Palaeontographica (Abteilung A: Paläozoologie-Stratigraphie) 157 (4/6): 175-200.

Bernacsek G.M. \& Dineley D.L. 1977. New acanthodians from the Delorme Formation (Lower Devonian) of N.W.T., Canada. Palaeontographica (Abteilung A: Paläozoologie- Stratigraphie) 158 (1-3): 1-25.

Bertin L. \& Arambourg C. 1958. Super-ordre des téléostéens (Teleostei). In: Grassé P.P. (ed.) Traité de Zoologie Tome 13 Fascicule 3: 2204-2500. Masson \& Cie, Paris.

Betancur-R. R., Broughton R.E., Wiley E.O., Carpenter K., Lopez J.A., Li C., Holcroft N.I., Arcila D., Sanciangco M., Cureton J., Zhang F., Buser T., Campbell M., Rowley T., Ballesteros J.A., Lu G., Grande T., Arratia G. \& Ortí G. 2013. The tree of life and a new classification of bony fishes. PLoS Currents Tree of Life. [28 April 2013] https://doi.org/10.1371/currents.tol.53ba26640df0ccaee75bb165c8c26288 
Betancur-R R., Wiley E.O., Bailly N., Acero A., Miya M., Lecointre G. \& Ortí G. 2016. Phylogenetic Classification of Bony Fishes. Version 4.

Available from https://sites.google.com/site/guilleorti/classification-v-4 [accessed 17 Jul. 2018].

Betancur-R R., Wiley E.O., Arratia G., Acero A., Bailly N., Miya M., Lecointre G. \& Ortí G. 2017. Phylogenetic classification of bony fishes. BMC Evolutionary Biology 17 (162): 1-41. https://doi.org/10.1186/s12862-017-0958-3

Billberg G.J. 1833. Om ichthyologien och beskrifning öfver några nya fiskarter af samkäksslägtet Syngnathus. Linnéska Samfundets Handlingar 1: 47-55. [In Swedish.] Available from https://archive.org/download/linnskasamfunde00samfgoog/linnskasamfunde00samfgoog.pdf [accessed 17 Jul. 2018].

Bleeker P. 1859. Enumeratio specierum piscium hucusque in Archipelago indico observatarum, adjectis habitationibus citationibusque, ubi descriptiones earum recentiores reperiuntur, nec non speciebus Musei Bleekeriani Bengalensibus, Japonicis, Capensibus Tasmanicisque. Acta Societatis Regiae Scientiarum Indo-Neêrlandicae [Verhandelingen der Natuurkundige Vereeniging in Nederlandsch Indië] 6. Natuurkundige Vereeniging in Nederlandsch Indië, Batavia.

Available from https://biodiversitylibrary.org/page/47271149 [accessed 25 Sep. 2018].

Blom H., Märss T. \& Miller C.G. 2002. Silurian and earliest Devonian birkeniid anaspids from the Northern Hemisphere. Transactions of the Royal Society of Edinburgh, Earth Sciences 92 [2001]: 263323. https://doi.org/10.1017/S0263593300000250

Blom H., Märss T. \& Miller C.G. 2003. A new birkeniid anaspid from the Upper Silurian of Skåne, south Sweden. GFF 125 (2): 57-61. https://doi.org/10.1080/11035890301252057

Blot J. 1966. Étude des Palaeonisciformes du Bassin Houiller de Commentry (Allier, France). Cahiers de Paléontologie, Édition Centre Nationale de la Recherche Scientifique, Paris.

Blot J. 1969. Les poissons fossiles du Monte Bolca, classés jusqu'ici dans les familles des Carangidae, Menidae, Ephippidae, Scatophagidae. Studi e ricerche sui giacimenti terziari di Bolca I: 1-525.

Blot J. 1976. Les Anguilliformes fossiles du Monte Bolca (Eocène inférieur). 26 ${ }^{\text {th }}$ Congress of European Ichthyologists, Paris, 1976. Revue des travaux de l'Institut des pêches maritimes 40 (3-4): 509-511.

Blot J. 1978. Les Apodes fossiles du Monte Bolca I. Miscellanea Paleontologica n. 1. Studi e ricerche sui giacimenti terziari di Bolca III: 1-260.

Blot J. 1981. La faune ichthyologique des gisements du Monte Bolca (Province de Vérone, Italie), Catalogue systématique présentant l'état actuel des recherches concernant cette fauna. Bulletin du Muséum national d'Histoire naturelle, Section C Sciences de la terre, paléontologie, géologie, minéralogie (série 4) 2 (4) [for 1980]: 339-396.

Available from http://bibliotheques.mnhn.fr/EXPLOITATION/infodoc/ged/viewportalpublished. ashx?eid=IFD_FICJOINT_BMCTE_S004_1980_T002_N004_1 [accessed 16 Sep. 2018].

Blot J. 1984. Les Apodes fossiles du Monte Bolca II. Miscellanea Paleontologica n. 2. Studi e ricerche sui giacimenti terziari di Bolca IV: 61-238.

Blot J. 1987. L'ordre des Pycnodontiformes. Miscellanea Paleontologica n. 3. Studi e ricerche sui giacimenti terziari di Bolca $\mathrm{V}: 1-211$.

Bock W. 1959. New Eastern American Triassic fishes and Triassic correlations. Geological Center Research Series 1: 1-184.

Bonaparte C.L. 1831. Saggio di una distribuzione metodica degli animali vertebrati, Roma. [Also 1832. Saggio d'una distribuzione .. vertebrati a sangue freddo. Presso Antonio Boulzaler, Rome; also in Giornale Arcadico di Scienze Lettere ed Arti 52 (1831): 155-189. Saggio di una 
distribuzione metodica deglianimali vertebrati (1832): 89-123; pagination in all 4 works differs.] https://doi.org/10.5962/bhl.title.48624

Bonaparte C.L. 1835. Prodromus systematis ichthyologiae. [Also published in 1840: Nuovi Annali delle Scienze naturali Bologna (Ser. 1) 2 (4): 181-196, 272-277.]

Bonaparte C.L. 1837. Synopsis vertebratorum systematis. [Also published in 1838: Nuovi Annali delle Scienze naturali Bologna (Ser. 1) 1 (2): 126-133.]

Bonaparte C.L. 1845. Specchio generale del sistema ittiologico. Atti della sesta Riunione degli Scienziati Italiani 6: 386-390. [Also published as a separate in 1845: Specchio generale dei sistemi erpetologico, anfibiologico ed ittiologico. Giacomo Pirola, Milano.]

Bonaparte C.L. 1846. Catalogo metodico dei pesci europei. Atti della Settima Adunanza degli Scienziati Italiani, Napoli, Part 2: 1-95. [Including Specchio generale del sistema ittiologico p. 3-9; also issued as separate, Napoli 1846.] https://doi.org/10.5962/bhl.title.59507

Bonaparte C.L. 1850a. Conspectus systematis ichtyologiae Caroli L. Bonaparte. Editio reformata 1850. Nuovi annali delle scienze naturali e rendiconto dei lavori dell'Accademia della Scienze dell'Instituto di Bologna con appendice agraria, Bologna (Ser. 3) 6: 453-456. [Also as a reprint.]

Bonaparte C.L. 1850b. Conspectus systematis ichthyologiae Caroli-Luciani Bonaparte, Classis V Pisces, Editio reformata. E. J. Brill, Lugduni Batavorum, 1 folio table.

Bonde N. 1966. The fishes of the MoClay Formation (Lower Eocene). Bulletin of the Geological Society of Denmark (Meddelelser fra Dansk Geologisk Forening) 16 (2): 198-202.

Borgen U.J. \& Nakrem H.A. 2016. Morphology, Phylogeny and Taxonomy of Osteolepiform Fish. Fossils and Strata Monograph Series Number 61, Wiley-Blackwell, New York.

https://doi.org/10.1002/9781119286448

Bouchard P., Bousquet Y., Davies A.E., Alonso-Zarazaga M.A., Lawrence J.F., Lyal C.H.C., Newton A.F., Reid C.A.M., Schmitt M., Ślipiński S.A. \& Smith A.B.T. 2011. Family-group names in Coleoptera (Insecta). ZooKeys 88 (Special Issue): 1-972. https://doi.org/10.3897/zookeys.88.807

Bouchet P. \& Rocroi J.-P. 2005. Classification and nomenclator of gastropod families. Malacologia 47 (1-2): 1-397. Available from https://www.biodiversitylibrary.org/page/25127194 [accessed 17 Jul. 2018].

Boulenger G.A. 1902a. Notes on the classification of teleostean fishes. III. On the systematic position of the genus Lampris, and on the limits and contents of the suborder Catosteomi. Annals and Magazine of Natural History (series 7) 10 (56): 147-152. https://doi.org/10.1080/00222930208678647

Boulenger G.A. 1902b. Notes on the classification of teleostean fishes. IV. On the systematic position of the Pleuronectidae. Annals and Magazine of Natural History (series 7) 10 (58): 295-304. https://doi.org/10.1080/00222930208678674

Boulenger G.A. 1904a. A synopsis of the suborders and families of teleostean fishes. Annals and Magazine of Natural History (series 7) 13 (75): 161-190. [1 March] https://doi.org/10.1080/00222930408678896

Boulenger G.A. 1904b. Chapters XXI-XXIII Teleostei: general characters, Malacopterygii ... Plectognathi. In: Harmer S.F. \& Shipley A.E. (eds) The Cambridge Natural History Volume VII: 541727. MacMillan, London. Available from https://www.biodiversitylibrary.org/item/15819 [accessed 17 Jul. 2018].

Bridge T.W. 1904. Fishes, Chapters V-XX. In: Harmer S.F. \& Shipley A.E. (eds) The Cambridge Natural History Volume VII: 141-537. MacMillan, London.

Available from https://www.biodiversitylibrary.org/item/15819 [accessed 17 Jul. 2018]. 
Broili F. 1933. Weitere Fische aus den Hunsrückschiefern. Sitzungsberichten der Bayerischen Akademie der Wissenschaften 1933: 269-313.

Bronn H.G. 1849. Index palaeontologicus, Zweite Abtheilung B Enumerator palaeontologicus. Schweizerbart, Stuttgart: 635-682. Available from https://www.biodiversitylibrary.org/item/182180 [accessed 17 Jul. 2018].

Brotzen F. 1933. Weigeltaspis nov. gen. und die Phylogenie der panzertragenden Heterostraci. Centralblatt für Mineralogie, Geologie und Paläontologie B 1933: 648-656.

Brough J. 1931. On fossil fishes from the Karroo System, and some general considerations on the bony fishes of the Triassic Period. Proceedings of the Zoological Society London 101 (1): 235-296. https://doi.org/10.1111/j.1469-7998.1931.tb06193.x

Brough J. 1939. The Triassic Fishes of Besano, Lombardy. British Museum (Natural History), London. Bryant W.L. 1933. The fish fauna of Bear Tooth Butte, Wyoming. Proceedings of the American Philosophical Society 72 (5): 285-314.

Bürgin T., Eichenberger U., Furrer H. \& Tschanz K. 1991. Die Prosanto-Formation - eine fischreiche Fossil-Lagerstätte in der Mitteltrias der Silvretta-Decke (Kanton Graubünden, Schweiz). Eclogae Geologicae Helvetiae 84 (3): 921-990. Available from https://www.e-periodica.ch/cntmng?pid=egh-001:1991:84::1128 [accessed 17 Jul. 2018].

Burrow C.J. \& Young G.C. 2005. The acanthodian fauna of the Craven Peaks Beds (Early to Middle Devonian), Western Queensland. Memoirs of the Queensland Museum 51 (1): 3-25. Available from http://www.qm.qld.gov.au/ /media/Documents/QM/About+Us/Publications/Memoirs+-+Nature/N51/ 51-1-burrows.pdf [accessed 17 Jul. 2018].

Burrow C.J., Turner S., Nowlan G.S. \& Denison R.H. 2013. Vertebrate microremains from the Late Silurian of Arisaig, Nova Scotia, Canada. Journal of Paleontology 87 (6): 1041-1059. https://doi.org/10.1666/12-154

Burton M. 1931. XIII Pisces. Zoological Record 67: 45.

Bystrow A.P. 1956. [Kolymaspis sibirica n. g., n. sp., a new representative of the Lower Devonian agnathous vertebrates]. Vestnik Leningradskogo Universiteta Geologiy 18: 5-13. [In Russian, author also seen as Bystrov.]

Camp C.L. \& Allison H.J. 1961. Bibliography of Fossil Vertebrates 1949-1953. Memoir of the Geological Society of America (84): 1-532.

Available from http://vertpaleo.org/Publications/Bibliography-of-Fossil-Vertebrates.aspx [accessed 17 Jul. 2018].

Camp C.L. \& Vanderhoof V.L. 1940. Bibliography of Fossil Vertebrates 1928-1933. Geological Society of America Special Papers (27): 1-504.

Available from http://vertpaleo.org/Publications/Bibliography-of-Fossil-Vertebrates.aspx [accessed 17 Jul. 2018].

Camp C.L., Welles S.P \& Green M. 1949. Bibliography of Fossil Vertebrates 1939-1943. Memoir of the Geological Society of America (37): 1-371.

Available from http://vertpaleo.org/Publications/Bibliography-of-Fossil-Vertebrates.aspx [accessed 17 Jul. 2018].

Campbell K.S.W.\&Barwick R.E. 1990. Paleozoic dipnoan phylogeny: functional complexes and evolution without parsimony. Paleobiology 16 (2): 143-169. https://doi.org/10.1017/S0094837300009854 
Cantalice K.M. \& Alvarado-Ortega J. 2016. Eekaulostomus cuevasae gen. and sp. nov., an ancient armored trumpetfish (Aulostomoidea) from Danian (Paleocene) marine deposits of Belisario Domínguez, Chiapas, southeastern Mexico. Palaeontologia Electronica 19.3.53A: 1-24. https://doi.org/10.26879/682

Cappetta H. 1974. Sclerorhynchidae nov. fam., Pristidae et Pristiophoridae: un exemple de parallélisme chez les sélaciens. Comptes rendus de l'Académie de Sciences Paris Série D 278 (2): 225-228.

Cappetta H. 1987. Chondrichthyes II: Mesozoic and Cenozoic Elasmobranchii. In: Schultze H.-P. (ed.) Handbook of Paleoichthyology Volume 3B: 1-193. Gustav Fischer Verlag, Stuttgart/New York.

Cappetta H. 1992a. Carcharhiniformes nouveaux (Chondrichthyes, Neoselachii) de l'Yprésien du Bassin de Paris. Geobios 25 (5): 639-646. https://doi.org/10.1016/0016-6995(92)80103-K

Cappetta H. 1992b. Nouveaux Rhinobatoidei (Neoselachii, Rajiformes) à denture spécialisée du Maastrichtien du Maroc. Remarques sur l'évolution dentaire des Rajiformes et des Myliobatiformes. Neues Jahrbuch für Geologie und Paläontologie, Abhandlungen 187 (1): 31-52.

Cappetta H. 2012. Chondrichthyes, Mesozoic and Cenozoic Elasmobranchii: Teeth. In: Schultze H.-P. (ed.) Handbook of Paleoichthyology Volume 3E. Verlag Dr. Friedrich Pfeil, Munich.

Cappetta H., Duffin C. \& Zidek J. 1993. Chondrichthyes. In: Benton M.J. (ed.) The Fossil Record 2: 593-609. Chapman \& Hall, London.

Carnevale G. \& Bannikov A.F. 2015. Pietschellidae fam. nov., a new family of miniature percomorph fishes from the Eocene of Bolca, with the description of a new genus and species. Miscellanea Paleontologica n. 13. Studi e ricerche sui giacimenti terziari di Bolca XVI: 17-26. Available from http://museodistorianaturale.comune.verona.it/media/_Musei/_StoriaNaturale/_Allegati/Miscellanea_ Paleontologica_N-13_017-026.pdf [accessed 17 Jul. 2018].

Casier E. 1946. La faune ichthyologique de l'Yprésien de la Belgique. Mémoires du Musée royal d'histoire naturelle de Belgique 104: 1-267.

Casier E. 1947a. Constitution et évolution de la racine dentaire des Euselachii. II. Étude comparative des types. Bulletin du Musée royal d'Histoire naturelle de Belgique 23 (14): 1-32. Available from http://www.vliz.be/imisdocs/publications/ocrd/291256.pdf [accessed 14 Sep. 2018].

Casier E. 1947b. Constitution et évolution de la racine dentaire des Euselachii. III. Évolution des principaux caractères morphologiques et conclusions. Bulletin du Musée royal d'Histoire naturelle de Belgique 23 (15): 1-45. Available from http://www.vliz.be/imisdocs/publications/ocrd/291257.pdf [accessed 14 Sep. 2018].

Casier E. 1966. Faune ichthyologique du London Clay. British Museum (Natural History), London.

Casier E. 1967. Poissons de l'Éocène inférieur de Katharinenhof-Fehmarn (Schleswig-Holstein). Bulletin de l'Institut royal des Sciences naturelles de Belgique (Sciences de la Terre) 43 (25): 1-23. Available from http://www.vliz.be/imisdocs/publications/ocrd/294738.pdf [accessed 14 Sep. 2018].

Castro F., Toledo C., de Sousa E.P. \& Medeiros M. 2004. Nova ocorrência de Asiatoceratodus (Osteichthyes, Dipnoiformes) na Formação Alcântara, Eocenomaniano da Bacia de São Luis, MA, Brasil. Revista Brasileira de Paleontología 7 (2): 245-248.

Available from http://www.sbpbrasil.org/revista/edicoes/7_2/castro.pdf [accessed 27 Jul. 2018].

Cavin L. 2017. Freshwater Fishes, 250 Million Years of Evolutionary History. ISTE Press, London.

Chabanaud P. 1937. Les téléostéens dyssymétriques du Mokattam inférieur de Tourah. Mémoires présentés de l'Institut d'Égypte 32: 1-121. 
Chalifa Y. 1989. Yabrudichthys and Serrilepis, two new genera of enchodontoids (Teleostei) from Lower Cenomanian beds of Ein-Yabrud, Israel. Israel Journal of Zoology 36 (1): 11-38.

Available from https://www.tandfonline.com/doi/abs/10.1080/00212210.1989.10688621 [accessed 17 Jul. 2018].

Chang K.-J. 1965. New antiarchs from the Middle Devonian of Yunnan. Vertebrata PalAsiatica 9 (1): 1-9. [In Chinese, with English summary, author also seen as Zhang G.-R.]. Available from http://www.ivpp.cas.cn/cbw/gjzdwxb/xbwzxz/200912/P020091218579407799199.pdf [accessed 17 Jul. 2018].

Chang M.-M. \& Chou C.-C. 1974. [Late Mesozoic fossil fishes from Province Chekiang]. Vertebrata PalAsiatica 12 (3): 183-186. [In Chinese, first author also seen as Zhang; second author also seen as Chow.] Available from http://www.ivpp.cas.cn/cbw/gjzdwxb/xbwzxz/200905/W020090813377540535384.pdf [accessed 17 Jul. 2018].

Chang M.-M. \& Chou C.-C. 1977. On late Mesozoic fossil fishes from Zhejiang Province, China. Memoirs of Institute of Vertebrate Palaeontology and Palaeoanthropology, Academia Sinica (12): 1-60. [In Chinese, with English summary.]

Choo B. 2012. Revision of the actinopterygian genus Mimipiscis (= Mimia) from the Upper Devonian Gogo Formation of Western Australia and the interrelationships of the early Actinopterygii. Earth and Environmental Science Transactions of the Royal Society of Edinburgh 102 (2): 77-104. https://doi.org/10.1017/S1755691011011029

Claypole E.W. 1885. On the recent discovery of pteraspidian fish in the Upper Silurian rocks of North America. The Quarterly Journal of the Geological Society of London 41: 48-64.

https://doi.org/10.1144/GSL.JGS.1885.041.01-04.10

Claypole E.W. 1892. On the structure of the American pteraspidian Palceaspis (Claypole), with remarks on the family. The Quarterly Journal of the Geological Society of London 48: 542-561. https://doi.org/10.1144/GSL.JGS.1892.048.01-04.40

Cloutier R. \& Ahlberg P.E. 1996. Morphology, Characters, and the Interrelationships of Basal Sarcopterygians. In: Stiassny M.L.J., Parenti L.R. \& Johnson G.D. (eds) Interrelationships of Fishes: 445-479. Academic Press, San Diego.

Cobbett A., Wilkinson M. \& Wills M.A. 2007. Fossils impact as hard as living taxa in parsimony analyses of morphology. Systematic Biology 56 (5): 753-766. https://doi.org/10.1080/10635150701627296

Cocchi I. 1864. Monografia dei Pharyngodopilidae nuova famiglia di pesci labroidi. Florence. [Also 1866 Annali del R. Museo di Fisica e Storia Naturale di Firenze (n.s.) 1.]

https://doi.org/10.5962/bhl.title.61006

Cockerell T.D.A. 1919. Some American Cretaceous fish-scales, with notes on the classification and distribution of Cretaceous fishes. Professional Papers of the United States Geological Survey 120: 165188.

Cockerell T.D.A. 1924. Fossils in the Ondai Sair Formation, Mongolia. Bulletin of the American Museum of Natural History 60 (VI): 129-144. Available from http://hdl.handle.net/2246/832 [accessed 27 Jul. 2018].

Cooper C.L. 1936. Actinopterygian jaws from the Mississippian Black Shales of the Mississippi Valley. Journal of Paleontology 10 (2): 92-94. Available from https://www.jstor.org/stable/1298344 [accessed 14 Sep. 2018]. 
Cope E.D. 1872. On the families of fishes of the Cretaceous Formation of Kansas. Proceedings of the American Philosophical Society 12 (86): 327-357. Available from http://www.jstor.org/stable/981715 [accessed 27 Aug. 2018].

Cope E.D. 1875. The Vertebrata of the Cretaceous formations of the West. Report of the United States Geological Survey of the Territories 2: 1-302.

Available from https://biodiversitylibrary.org/page/40586457 [accessed 14 Sep. 2018].

Cope E.D. 1877a. On the Vertebrata of the Bone Bed in Eastern Illinois. Proceedings of the American Philosophical Society 17 (100): 327-357. Available from http://www.jstor.org/stable/982278 [accessed 27 Aug. 2018].

Cope E.D. 1877b. On the genus Erisichthe. Bulletin of the United States Geological and Geographical Survey of the territories 3 (35): 821-823. Available from https://biodiversitylibrary.org/page/31646549 [accessed 14 Sep. 2018].

Cope E.D. 1877c. Geology and Palaeontology. The American Naturalist 11 (9): 568-570. Available from http://www.jstor.org/stable/2448196 [accessed 17 Jul. 2018].

Cope E.D. 1886. An interesting connecting genus of Chordata. The American Naturalist 20 (12): 10271031. Available from http://www.jstor.org/stable/2450501 [accessed 17 Jul. 2018].

Cope E.D. 1887. Geology and Palaeontology: Zittel's Manual of Palaeontology. The American Naturalist 21 (11): 1014-1020. Available from http://www.jstor.org/stable/2450870 [accessed 27 Jul. 2018].

Cope E.D. 1889. Synopsis of the families of Vertebrata. The American Naturalist 23 (274): 849-877. Available from http://www.jstor.org/stable/2451539 [accessed 17 Jul. 2018].

Cox C.B. \& Hutchinson P. 1991. Fishes and amphibians from the Late Permian Pedra de Fogo Formation of northern Brazil. Palaeontology 34 (3): 561-573. Available from https://www.palass.org/publications/palaeontology-journal/archive/34/3/article_pp561-573 [accessed 27 Jul. 2018].

Cragin F.W. 1901. A study of some teleosts from the Russell Substage of the Platte Cretaceous Series. Colorado College Studies 9: 25-44. [May.] Available from https://biodiversitylibrary.org/page/29946271 [accessed 14 Sep. 2018].

Crook A.R. 1892. Ueber einige fossile Knochenfische aus der mittleren Kreide von Kansas. Palaeontographica 39: 107-124.

Available from https://biodiversitylibrary.org/page/33297206 [accessed 14 Sep. 2018].

Cuny G., Suteethorn V., Kamha S. \& Buffetaut E. 2008. Hybodont sharks from the lower Cretaceous Khok Kruat Formation of Thailand, and hybodont diversity during the Early Cretaceous. In: Cavin L., Longbottom A.E. \& Richter M. (eds) Fishes and the Break-up of Pangaea: 93-107. Geological Society of London, Special Publication 295.

Dames W. 1888. "K.A. Zittel: Handbuch der Palaeontologie. 1. Abtheil. Palaeozoologie. III. Bd. 1. Lief. München 1887. $8^{\circ}$. 256 S. 266 Textfiguren". Neues Jahrbuch für Mineralogie, Geologie und Paläontologie 1888 (2): 128-132.

Available from https://biodiversitylibrary.org/page/44314187 [accessed 14 Sep. 2018].

Daniltshenko P.G. 1960. Kostistye ryby maikopskikh otlozhenii Kavkaza [Bony fishes of the Maikop deposits of the Caucasus]. Trudy Paleontologicheskogo Instituta (Akademiya Nauk SSSR) [Transactions of the Paleontological Institute of the Academy of Sciences of the USSR] 78: 1-208. [Author also seen as Danil'chenko or Danilchenko, English translation by the Israel Program for Scientific Translations 1967: 1-247.] 
Daniltshenko P.G. 1964. Teleostei. In: Obruchev D.V. (ed.) Osnovy paleontologii [Fundamentals of Paleontology] Vol. XI. Agnatha, Pisces: 396-484. Izdatel'stvo 'Nauka', Moscow. [In Russian, translated 1967, Fundamentals of Paleontology, Vol. XI. Agnatha, Pisces. Israel Program for Scientific Translations, Jerusalem.]

Daniltshenko P.G. 1968. [Fishes from the Upper Paleocene of Turkmenia]. In: Obruchev D.V. (ed.) Ocherki po filogenii i sistematike iskopayemykh ryb i beschelyustnykh [Outlines on the Phylogeny and Systematics of Fossil Fishes and Agnathans]: 113-156. Nauka, Moscow. [In Russian.]

Daniltshenko P.G. 1980. [Orders Elopiformes, Clupeiformes, Salmoniformes, Myctophiformes, Lampridiformes, Beryciformes, Zeiformes, Perciformes, Echeneiformes, Tetraodontiformes]. In: Novitskaya L.I. (ed.) Iskopaemye kostistye ryby SSSR [Fossil Teleost Fishes of the USSR]: 6-28, 45-50, 91-96, 99-104, 115-169, 173-174. Nauka, Moscow. [In Russian.]

Dartevelle E. \& Casier E. 1943. Les poissons fossiles du Bas-Congo et des régions voisines. (Première Partie). Annales du Musée du Congo belge A (Minéralogie, géologie, paléontologie) (série 3) 2 (1): $1-200$.

Dartevelle E. \& Casier E. 1949. Les poissons fossiles du Bas-Congo et des régions voisines. (Deuxième Partie). Annales du Musée du Congo belge A (Minéralogie, géologie, paléontologie) (série 3) 2 (2): 201-255.

Davesne D., Friedman M., Barriel V., Lecointre G., Janvier Ph., Gallut C. \& Otero O. 2014. Early fossils illuminate character evolution and interrelationships of Lampridiformes (Teleostei, Acanthomorpha). Zoological Journal of the Linnean Society 172 (2): 475-498. https://doi.org/10.1111/zoj.12166

David L.R. 1943. Miocene Fishes of Southern California. Geological Society of America Special Paper 43, Geological Society of America. Available from https://www.biodiversitylibrary.org/page/34154312 [accessed 17 Jul. 2018].

Davis A.C. 1994. Catalogue of Type, Figured and Cited Specimens in the Commonwealth Palaeontological Collection VERTEBRATA. Department of Geology, Faculty of Sciences, Australian National University, Canberra. Available from https://d28rz98at9flks.cloudfront.net/14735/Rec1994_032.pdf [accessed 15 Sep. 2018].

Davis J.W. 1883. Notes on the occurrence of fossil fish remains in the Carboniferous limestone series of Yorkshire. Proceedings of the Yorkshire Geological and Polytechnic Society (New Series) 8 [for 1882]: 39-63. Available from https://biodiversitylibrary.org/page/47755196 [accessed 15 Sep. 2018].

De Koninck see Koninck L.-G. de.

Dean B. 1894. Contributions to the morphology of Cladoselache (Cladodus). Journal of Morphology 9 (1): 87-114. https://doi.org/10.1002/jmor.1050090103

Dean B. 1901. Palaeontological notes III. Further notes on the relationships of the Arthrognathi. Memoirs of the New York Academy of Sciences 2 (3): 110-123.

Dean B. 1909. Studies on fossil fishes (sharks, chimaeroids and arthrodires). Memoirs of the American Museum of Natural History 9 (V): 209-287. Available from http://hdl.handle.net/2246/57 [accessed 17 Jul. 2018].

Delsate D. 2001. L'ichthyofaune du Pliensbachien (Jurassique inférieur) de Lorraine et des Ardennes (France): premiers résultats. Bulletin de l'Académie lorraine des Sciences 40 (1/2): 47-69.

Delsate D. \& Candoni L. 2001. Description de nouveaux morphotypes dentaires de Batomorphii toarciens (Jurassique inférieur) du Bassin de Paris: Archaeobatidae nov. fam. Bulletin de la Société des naturalistes luxembourgeois 2001: 131-144. 
Denison R.H. 1951. Evolution and classification of the Osteostraci. Fieldiana, Geology 11 (3-4): 156196. https://doi.org/10.5962/bhl.title.3286

Denison R.H. 1975. Evolution and classification of Placoderm fishes. Breviora (432): 1-24. Available from https://www.biodiversitylibrary.org/page/6590294 [accessed 27 Jul. 2018].

Denison R.H. 1978. Placodermi. In: Schultze H.-P. (ed.) Handbook of Paleoichthyology Volume 2: 1-128. Gustav Fischer Verlag, Stuttgart / New York.

Denison R.H. 1979. Acanthodii. In: Schultze H.-P. (ed.) Handbook of Paleoichthyology Volume 5: 1-62. Gustav Fischer Verlag, Stuttgart / New York.

Denison R.H. 1984. Further consideration of the phylogeny and classification of the order Arthrodira (Pisces: Placodermi). Journal of Vertebrate Paleontology 4 (3): 396-412.

Available from http://www.jstor.org/stable/4523000 [accessed 17 Jul. 2018].

Dennis K. \& Miles R.S. 1979. Eubrachythoracid arthrodires with tubular rostral plates from Gogo, Western Australia. Zoological Journal of the Linnean Society 67 (4): 297-328.

https://doi.org/10.1111/j.1096-3642.1979.tb01118.x

Dick J.R.F. 1981. Diplodoselache woodi gen. and sp. nov., an early Carboniferous shark from the Midland Valley of Scotland. Transactions of the Royal Society of Edinburgh, Earth Sciences 72 (2): 99-113. https://doi.org/10.1017/S0263593300009937

Dineley D.L. 1955. Notes on the genus Corvaspis. Proceedings of the Royal Society of Edinburgh, Section B 65 (2): 166-181. https://doi.org/10.1017/S0080455X00012091

Dineley D.L. \& Loeffler E.J. 1976. Ostracoderm Faunas of the Delorme and Associated Siluro-Devonian Formations, North West Territories, Canada. Special Papers in Palaeontology 18, The Palaeontological Association, London.

Duffin C.J. 1998. Ostenoselache stenosoma n. g. n. sp., a new neoselachian shark from the Sinemurian (Early Jurassic) of Osteno (Lombardy, Italy). Paleontologia Lombarda (nuova serie) 9: 3-27.

Duffin C.J. \& Ward D.J. 1983. Neoselachian sharks' teeth from the Lower Carboniferous of Britain and the Lower Permian of the USA. Palaeontology 26 (1): 93-110.

Available from https://www.palass.org/sites/default/files/media/publications/palaeontology/volume_ 26/vol26_part1_pp93-110.pdf [accessed 17 Jul. 2018].

Duffin C.J. \& Ward D.J. 1993. The Early Jurassic palaeospinacid sharks of Lyme Regis, southern England. Professional Paper of the Geological Survey of Belgium 264: 53-102.

Dupret V. \& Zhu M. 2008. The earliest phyllolepid (Placodermi, Arthrodira) from the Late Lochkovian (Early Devonian) of Yunnan (South China). Geological Magazine 145 (2): 257-278. https://doi.org/10.1017/S0016756807004207

Dupret V., Phuong T.H., Thanh T.-D., Phong N.D., Janvier Ph. \& Clément G.A. 2011. The skull of Hagiangella goujeti Janvier, 2005, a high-crested acanthothoracid (Vertebrata, Placodermi) from the lower Devonian of northern Vietnam. Journal of Vertebrate Paleontology 31 (3): 531-538. https://doi.org/10.1080/02724634.2011.558148

Eastman C.R. 1898. Some new points in dinichthyid osteology. Proceedings of the American Association for the Advancement of Science 1898: 371-372.

Eastman C.R. 1902. On the genus Peripristes, St. John. Geological Magazine (Decade 4) 9: 388-391. Available from https://biodiversitylibrary.org/page/30545112 [accessed 17 Jul. 2018].

Eastman C.R. 1907. Devonic Fishes of the New York Formations. New York State Museum, Memoir 10. New York State Education Dept, Albany. https://doi.org/10.5962/bhl.title.54661 
Eastman C.R. 1917. Fossil fishes in the collection of the United States National Museum. Proceedings of the United States National Museum 52 (no. 2177): 235-304.

https://doi.org/10.5479/si.00963801.52-2177.235

Ebert M. 2016. Pycnodontidae (Actinopterygii) in the late Jurassic: 2) Turboscinetes gen. nov. in the Solnhofen Archipelago (Germany) and Cerin (France). Archaeopteryx 33: 12-53.

Ebert M. 2018. Cerinichthys koelblae, gen. et sp. nov., from the Upper Jurassic of Cerin, France, and its phylogenetic setting, leading to a reassessment of the phylogenetic relationships of Halecomorphi (Actinopterygii). Journal of Vertebrate Paleontology 38 (1): [1-22] e1420071-15.

https://doi.org/10.1080/02724634.2017.1420071

Edgecombe G.D. 2010. Palaeomorphology: fossils and the inference of cladistic relationships. Acta Zoologica 91: 72-80. https://doi.org/10.1111/j.1463-6395.2009.00426.x

Eichwald C.E. von 1846. Nachtrag zu der Beschreibung der Fische des devonischen Systems aus der Gegend von Pawlowsk. Bulletin de la Société impériale des naturalistes de Moscou 19: 1-44.

Available from https://biodiversitylibrary.org/page/44106381 [accessed 15 Sep. 2018].

Eichwald C.E. von 1860. Lethaea rossica, ou Paléontologie de la Russie, Volume 1 pt. 2. Classe septième. Poissons: 1493-1607. Schweizerbart, Stuttgart.

Available from https://www.biodiversitylibrary.org/item/112874 [accessed 17 Jul. 2018].

Eichwald C.E. von 1868. Lethaea rossica, ou Paléontologie de la Russie, Volume 2 pt. 2. Classi huitième. Poissons: 1195-1265. Schweizerbart, Stuttgart.

Available from https://www.biodiversitylibrary.org/item/112228 [accessed 27 Jul. 2018].

Elliott D.K. 1984. A new subfamily of Pteraspididae (Agnatha, Heterostraci) from the Upper Silurian and Lower Devonian of Arctic Canada. Palaeontology 27 (1): 169-197. Available from https://www.palass.org/sites/default/files/media/publications/palaeontology/volume_27/ vol27_part1_pp169-197.pdf [accessed 17 Jul. 2018].

Elliott D.K. 2016. The Boothiaspidinae, a new agnathan subfamily (Heterostraci, Cyathaspididae) from the late Silurian and Early Devonian of the western United States and the Canadian Arctic. Journal of Paleontology 90 (6): 1212-1224. https://doi.org/10.1017/jpa.2016.113

Elliott D.K. \& Swift S. 2010. A new species of Ariaspis (Agnatha, Heterostraci) from the Late Silurian of the Canadian Arctic. Journal of Vertebrate Paleontology 30 (6): 1874-1878.

https://doi.org/10.1080/02724634.2010.521900

Eschmeyer W.N., Fricke R. \& van der Laan R. (eds) 2018. Catalog of Fishes: Genera, Species, References. Electronic version.

Available from http://researcharchive.calacademy.org/research/ichthyology/catalog/fishcatmain.asp [accessed 31 May 2018].

Estes R. 1969a. Studies on fossil phyllodont fishes, interrelationships and evolution in the Phyllodontidae (Albuloidei). Copeia 1969 (2): 317-331. https://doi.org/10.2307/1442082

Estes R. 1969b. Two new Late Cretaceous fishes from Montana and Wyoming. Breviora (335): 1-15. Available from https://www.biodiversitylibrary.org/page/4293637 [accessed 17 Jul. 2018].

Fielitz C. 2004. The phylogenetic relationships of the $†$ Enchodontidae (Teleostei: Aulopiformes). In: Arratia G., Wilson M.V.H. \& Cloutier R. (eds) Recent Advances in the Origin and Early Radiation of Vertebrates: 619-634. Verlag Dr. Friedrich Pfeil, Munich.

Fierstine H.L. \& Weems R.E. 2009. Paleontology of the Oligocene Ashley and Chandler Bridge Formations of South Carolina 4: analysis and new records of billfishes (Perciformes: Xiphioidei). Palaeo Ichthyologica 11: 43-88. 
Fitzinger L.J.F. 1873. Versuch einer natürlichen Classification der Fische. Sitzungsberichte der Mathematisch-Naturwissenschaftlichen Classe der Kaiserlichen Akademie der Wissenschaften 67 (1): 5-58. Available from https://biodiversitylibrary.org/page/6313068 [accessed 15 Sep. 2018].

Forey P.L. 1977. The osteology of Notelops Woodward, Rhacolepis Agassiz and Pachyrhizodus Dixon (Pisces: Teleostei). Bulletin of the British Museum (Natural History), Geology 28 (2): 123-204. Available from https://biodiversitylibrary.org/page/2251874 [accessed 15 Sep. 2018].

Forey P.L. 1998. History of the Coelacanth Fishes. Chapman and Hall, London.

Fournier G. \& Pruvost P. 1928. Description des poissons elasmobranches du marbre noir de Denée. Mémoires de la Société géologique du Nord 9 (2): 1-23.

Fowler H.W. 1911. A description of the fossil fish remains of the Cretaceous, Eocene and Miocene formation of New Jersey. Cretaceous and Tertiary Fish. Bulletin of the Geological Survey of New Jersey (4): 22-192. https://doi.org/10.5962/bhl.title.63936

Fowler H.W. 1947. New taxonomic names of fish-like vertebrates. Notulae Naturae (Philadelphia) (187): 1-16. [21 February]

Fowler H.W. 1951. Some new or emended names of fish-like vertebrates. The Fish Culturist 30 (10, suppl. 1): 1-4. [31 May]

Fowler H.W. 1964. A Catalog of World Fishes [Part I]. Quarterly Journal of the Taiwan Museum (Taipei) 17 (3-4): 1-62.

Fowler H.W. 1965a. A Catalog of World Fishes [Part II]. Quarterly Journal of the Taiwan Museum (Taipei) 18 (1-2): 137-202.

Fowler H.W. 1965b. A Catalog of World Fishes [Part III]. Quarterly Journal of the Taiwan Museum (Taipei) 18 (3-4): 341-397.

Fowler H.W. 1966a. A Catalog of World Fishes [Part IV]. Quarterly Journal of the Taiwan Museum (Taipei) 19 (1-2): 75-139.

Fowler H.W. 1966b. A Catalog of World Fishes [Part V]. Quarterly Journal of the Taiwan Museum (Taipei) 19 (3-4): 303-371.

Fowler H.W. 1971a. A Catalog of World Fishes, Volume II. Quarterly Journal of the Taiwan Museum (Taipei) 23 (3-4): 201-251.

Fowler H.W. 1971b. A Catalog of World Fishes [Part XIV]. Quarterly Journal of the Taiwan Museum (Taipei) 24 (1-2): 1-58.

Fowler H.W. 1971c. A Catalog of World Fishes [Part XV]. Quarterly Journal of the Taiwan Museum (Taipei) 24 (3-4): 365-409.

Fowler H.W. 1973. A Catalog of World Fishes [Part XIX]. Quarterly Journal of the Taiwan Museum (Taipei) 26 (3-4): 217-346.

Fraas E. 1896. Neue Selachier-Reste aus dem oberen Lias von Holzmaden in Württemberg. 1. Hybodus Hauffianus E. Fraas (= Hybodus reticulatus Quenstedt non Agassiz). II. Palaeospinax smith-woodwardii. Jahreshefte des Vereins vaterländische Naturkunde in Württemberg 52: 1-25.

Available from https://biodiversitylibrary.org/page/7921725 [accessed 15 Sep. 2018].

Frič A. 1889. Fauna der Gaskohle und der Kalksteine der Permformation Böhmens. Band II. Frič, Prague. Available from https://www.biodiversitylibrary.org/item/51308 [accessed 17 Jul. 2018].

Frič A. 1893. Fauna der Gaskohle und der Kalksteine der Permformation Böhmens. Band III/2: 49-80. Frič, Prague. Available from https://biodiversitylibrary.org/page/15676679 [accessed 17 Jul. 2018]. 
Frič A. 1894. Fauna der Gaskohle und der Kalksteine der Permformation Böhmens. Band III/3: 81-104. Frič, Prague. Available from https://biodiversitylibrary.org/page/15676757 [accessed 17 Jul. 2018].

Frič A. 1895. Fauna der Gaskohle und der Kalksteine der Permformation Böhmens. Band III/4: 105132. Frič, Prague. Available from https://biodiversitylibrary.org/page/15676823 [accessed 17 Jul. 2018].

Fritzsche R.A. 1980. Revision of the eastern Pacific Syngnathidae (Pisces: Syngnathiformes), including both recent and fossil forms. Proceedings of the California Academy of Sciences (series 4), 42 (6): 181-227. Available from https://www.biodiversitylibrary.org/page/15774907 [accessed 27 Jul. 2018].

Frizzell D.L. 1965. Otoliths of new fish (Vorhisia vulpes, n. gen., n. sp. Siluroidei?) from Upper Cretaceous of South Dakota. Copeia 1965 (2): 178-181. https://doi.org/10.2307/1440721

Gagnier P.-Y., Hanke G.F. \& Wilson M.V.H. 1999. Tetanopsyrus lindoei gen. et sp. nov., an Early Devonian acanthodian from the Northwest Territories, Canada. Acta Geologica Polonica 49 (2): 81-96. Available from https://geojournals.pgi.gov.pl/agp/article/view/10325 [accessed 17 Jul. 2018].

Gagnier P.-Y. \& Wilson M.V.H. 1996. Early Devonian acanthodians from northern Canada. Palaeontology 39 (2): 241-258.

Available from https:/www.palass.org/sites/default/files/media/publications/palaeontology/volume_39/ vol39_part2_pp241-258.pdf [accessed 17 Jul. 2018].

Gardiner B.G. 1960. A revison of certain actinopterygian and coelacanth fishes, chiefly from the Lower Lias. Bulletin of the British Museum (Natural History), Geology 4 (7): 239-384.

Available from https://biodiversitylibrary.org/page/36358938 [accessed 27 Aug. 2018].

Gardiner B.G. 1963. Certain palaeoniscid fishes and the evolution of the snout in actinopterygians. Bulletin of the British Museum (Natural History), Geology 8 (6): 255-325.

Available from https://www.biodiversitylibrary.org/page/36370884 [accessed 17 Jul. 2018].

Gardiner B.G. 1967. Further notes on palaeoniscid fishes with a classification of the Chondrostei. Bulletin of the British Museum (Natural History), Geology 14 (5): 143-206.

Available from https://www.biodiversitylibrary.org/page/36368846 [accessed 17 Jul. 2018].

Gardiner B.G. 1969. New palaeoniscoid fish from the Witteberg series of South Africa. Zoological Journal of the Linnean Society 48 (4): 423-452. https://doi.org/10.1111/j.1096-3642.1969.tb00722.x

Gardiner B.G. 1984. The relationships of the palaeoniscid fishes, a review based on new specimens of Mimia and Moythomasia from the Upper Devonian of Western Australia. Bulletin of the British Museum (Natural History), Geology 37: 173-428.

Available from https://www.biodiversitylibrary.org/page/2358825 [accessed 17 Jul. 2018].

Gardiner B.G. 1985. Actinopterygian fish from the Dinantian of Foulden, Berwickshire, Scotland. Transactions of the Royal Society of Edinburgh, Earth Sciences 76 (1): 61-66.

https://doi.org/10.1017/S0263593300010312

Gardiner B.G. 1993a. Placodermi. In: Benton M.J. (ed.) The Fossil Record 2: 583-588. Chapman and Hall, London.

Gardiner B.G. 1993b. Osteichthyes: basal actinopterygians. In: Benton M.J. (ed.) The Fossil Record 2: 611-619. Chapman and Hall, London.

Gardiner B.G. \& Miles R.S. 1990. A new genus of eubrachythoracid arthrodire from Gogo, Western Australia. Zoological Journal of the Linnean Society 99 (2): 159-204.

https://doi.org/10.1111/j.1096-3642.1990.tb00566.x

Gardiner B.G. \& Schaeffer B. 1989. Interrelationships of lower actinopterygian fishes. Zoological Journal of the Linnean Society 97 (2): 135-187. https://doi.org/10.1111/j.1096-3642.1989.tb00550.x 
Gaudant J. 1967a. Sur la nécessité d'une subdivision du genre Anaethalion White (poisson téléostéen). Compte rendu sommaire des séances de la Société géologique de France: 308-310.

Gaudant J. 1967b. Essai de révision taxonomique des 'Pholidophorus' de Cerin (Ain). Compte rendu sommaire des séances de la Société géologique de France: 373-374.

Gaudant J. 1968. Recherches sur l'anatomie et la position systématique du genre Lycoptera (poisson téléostéen). Mémoires de la Société géologique de France (nouvelles séries) 47 (109): 1-41.

Gaudant J. 1978. Essai de révision taxonomique des 'Pholidophorus' (poissons actinoptérygiens) du Jurassique supérieur de Cerin (Ain). Nouvelles archives du Muséum d'Histoire naturelle de Lyon 16: $101-121$.

Gaudant M. 1976. Sur la création d'un ordre nouveau de Poissons téléostéens, les Pattersonichthyiformes, et sur ses relations phylogénétiques. Comptes rendus hebdomadaires des séances de l'Académie des Sciences série D 283: 1629-1631. Available from https://gallica.bnf.fr/ark:/12148/bpt6k5768551v/f415. image.r=gaudantPattersonichthyiformes\%20gaudant\%20Pattersonichthyiformes [accessed 15 Sep. 2018].

Gaudant M. 1978a. Nouvelles observations sur l'anatomie et sur la position systématique d'un poisson teléosteen du cénomanien du Liban: Pateroperca Woodward. Geobios 11 (2): 189-210. https://doi.org/10.1016/S0016-6995(78)80088-6

Gaudant M. 1978b. Contribution à une révision des poissons crétacés du Jbel Tselfat (Rides Prérifaines, Maroc). Notes et Mémoires du Service géologique du Maroc 39: 79-124.

Gaudant M. 1978c. Recherches sur l'anatomie et la systématique des Cténothrissiformes et des Pattersonichthyiformes (Poissons Téléostéens) du Cénomanien du Liban. Mémoires du Muséum national d'Histoire naturelle, Série C, Sciences de la Terre 41: 1-124.

Gaudant M. 1979. Recherches sur les relations phylogénétiques de certains poissons Eurypterygii du Crétacé de la Mésogée occidentale. Comptes rendus hebdomadaires des séances de l'Académie des Sciences Série D 288: 1047-1050.

Available from https://gallica.bnf.fr/ark:/12148/bpt6k5497402t/f303.image.r=Eurypterygii [accessed 15 Sep. 2018].

Gayet M. 1980a. Recherches sur l'ichthyofaune Cénomanienne des Monts de Judée: les acanthoptérygiens. Annales de Paléontologie (Vertebrés) 66: 75-120.

Gayet M. 1980b. Contribution a l'étude anatomique et systématique des poissons cénomaniens du Liban, anciennement placés dans les acanthoptérygiens. Mémoires du Muséum national d'Histoire naturelle, Série C, Sciences de la Terre 44: 1-149.

Gayet M. 1982a. Essai de définition des relations phylogénétiques des Holocentroidea nov. et des Trachichthyoidea nov. (Pisces, Acanthopterygii, Béryciformes). Bulletin du Muséum national d'histoire naturelle, Section C, Sciences de la Terre, paléontologie, géologie, minéralogie (série 4) 4 (1-2): 2141. Available from http://bibliotheques.mnhn.fr/EXPLOITATION/infodoc/ged/viewportalpublished. ashx?eid=IFD_FICJOINT_BMCTE_S004_1982_T004_N001_1 [accessed 16 Sep. 2018].

Gayet M. 1982b. Étude anatomique et systématique des acanthoptérygiens du Sénonien de Sahel-Alma (Liban). Palaeontographia Italica 72: 98-136. 
Gayet M. 1985a. Gonorhynchiforme nouveau du Cénomanien inférieur marin de Ramallah (Monts de Judée): Judeichthys haasi nov. gen. nov. sp. (Teleostei, Ostariophysi, Judeichthyidae nov. fam.). Bulletin du Muséum national d'Histoire naturelle, Section C, Sciences de la Terre, paléontologie, géologie, minéralogie (série 4) 7 (1): 65-85. Available from http://bibliotheques.mnhn.fr/EXPLOITATION/ infodoc/ged/viewportalpublished.ashx?eid=IFD_FICJOINT_BMCTE_S004_1985_T007_N001_1 [accessed 16 Sep. 2018].

Gayet M. 1985b. Rôle de l'évolution de l'appareil de Weber dans la phylogénie des Ostariophysi suggéré par un nouveau Characiforme du Cénomanien supérieur marin du Portugal. Comptes Rendus de l'Académie des Sciences (série 2) 300 (17): 895-898.

Available from https://gallica.bnf.fr/ark:/12148/bpt6k62984305/f907.item.r=ostariophysi [accessed 15 Sep. 2018].

Gayet M. 1985c. Contribution à l'étude anatomique et systématique de l'ichthyofaune cénomanienne du Portugal. III. Complément à l'étude des Ostariophysaires. Comunicações dos Serviços Geológicos de Portugal 71 (1): 91-117.

Gayet M. 1988. Le plus ancien crâne de Siluriforme: Andinichthys bolivianensis nov. gen., nov. sp. (Andinichthyidae nov. fam.) du Maastrichtien de Tiupampa (Bolivie). Comptes Rendus de l'Académie des Sciences (série 2) 307: 833-836. Available from https://gallica.bnf.fr/ark:/12148/bpt6k6327064k/ f847.image.r=Andinichthys\%20bolivianensis?rk=64378;0 [accessed 15 Sep. 2018].

Gayet M. 1993. Gonorhynchoidei du Crétacé supérieur marin du Liban et relations phylogénétiques des Charitosomidae nov. fam. Documents des Laboratoires de Géologie de Lyon (126): 1-131.

Geinitz B. 1860. Zur Fauna des Rothliegenden und Zechsteins. Zeitschrift der Deutschen Geologischen Gesellschaft 12 (3): 467-470. Available from https://biodiversitylibrary.org/page/34776507 [accessed 15 Sep. 2018].

Giebel C.G. 1846. Paläozoologie, Entwurf einer systematischen Darstellung der Fauna der Vorwelt. Nulandt'sche Buchandlung, Merseburg. https://doi.org/10.5962/bhl.title.39515

Giebel C.G. 1847. Fauna der Vorwelt mit steter Berücksichtigung der lebenden Thiere. Band I Wirbelthiere Vierte Klasse Pisces. Brockhaus, Leipzig.

Available from https://www.biodiversitylibrary.org/item/74314 [accessed 17 Jul. 2018].

Gill T.N. 1872. Arrangement of the Families of Fishes, or Classes Pisces, Marsipobranchii, and Leptocardii. Smithsonian Miscellaneous Collections 247. Smithsonian Institution, Washington. https://doi.org/10.5962/bhl.title.18974

Gill T.N. 1884. On the mutual relations of the hemibranchiate fishes. Proceedings of the Academy of Natural Sciences of Philadelphia 36: 154-166.

Available from http://www.jstor.org/stable/4060994 [accessed 17 Jul. 2018].

Gill T.N. 1888. Some extinct scleroderms. American Naturalist 22 (257): 446-448.

Available from https://www.jstor.org/stable/2451210?seq=3\#metadata_info_tab_contents [accessed 17 Jul. 2018].

Gill T.N. 1889. Articles. In: The Century Dictionary. An Encyclopedic Lexicon of the English Language Volume 3: 2423-3556. The Century Co., New York.

Available from https://biodiversitylibrary.org/page/52494275 [accessed 15 Sep. 2018].

Gill T.N. 1893. Families and subfamilies of fishes. Memoirs of the National Academy of Science 6 (6): 127-138. https://doi.org/10.5962/bhl.part.6303

Gill T.N. 1896a. Note on the Devonian Palaeospondylus. Science (New Series) 4 (79): 10-11.

Available from http://www.jstor.org/stable/1623766 [accessed 17 Jul. 2018]. 
Gill T.N. 1896b. Notes on the synonymy of the Torpedinidae or Narcobatidae. Proceedings of the United States National Museum 18 (1050): 161-165. https://doi.org/10.5479/si.00963801.18-1050.161

Ginter M., Hairapetian V. \& Klug C. 2002. Famennian chondrichthyans from the shelves of North Gondwana. Acta Geologica Polonica 52 (2): 169-215.

Available from https://geojournals.pgi.gov.pl/agp/article/view/10071/8601 [accessed 17 Jul. 2018].

Ginter M., Hampe O. \& Duffin C.J. 2010. Chondrichthyes, Paleozoic Elasmobranchii: Teeth. In: Schultze H.-P. (ed.) Handbook of Paleoichthyology Volume 3D: 1-168. Verlag Dr. Friedrich Pfeil, Munich.

Ginter M., Liao J.-C. \& Valenzuela-Rios J.I. 2008. New data on chondrichthyan microremains from the Givetian of the Renanué section in the Aragonian Pyrenees (Spain). Acta Geologica Polonica 58 (2): $165-172$.

Available from https://geojournals.pgi.gov.pl/agp/article/view/9993/8523 [accessed 17 Jul. 2018].

Gistel J.N.F.X. 1848. Naturgeschichte des Tierreichs für höhere Schulen. Scheitlin \& Krais, Stuttgart.

Glickman L.S. 1956. [About the phylogeny of the genus Anacorax]. Doklady Akademii Nauk SSSR / Comptes rendus de l'Académie des sciences de l'URSS (N.S.) 109 (5): 1049-1052. [In Russian, author also seen as Glückman, Glikman, Ghlikman, Glyckman or Glykman.]

Glickman L.S. 1957. O gheneticheskoi svyazi semeistv Lamnidae I Odontaspidae I novuikh rodakh verkhnemelovuikh Lamnid [About the genetic link between the families Lamnidae and Odontaspidae and some new genera of Upper Cretaceous lamnids]. Trudy geologicheskogo muzeya imeni A.P. Karpinskogho. Academy of Sciences of the USSR, Moscow / Leningrad 1: 110-117. [In Russian.]

Glickman L.S. 1958. O tempakh evolyutsii lamnoidnykh akul [Rates of evolution in lamnoid sharks]. Doklady Akademii Nauk SSSR / Comptes rendus de l'Académie des sciences de l'URSS (N.S.) 123 (3): 568-572. [In Russian.]

Glickman L.S. 1964a. Akuly paleogena i ikh stratigraficheskoe znachenie [The sharks of the Paleogene and their stratigraphical significance]. Izdatel'stvo 'Nauka', Moscow/Leningrad. [In Russian.]

Glickman L.S. 1964b. Subclass Elasmobranchii. Selachian fishes. In: Obruchev D.V. (ed.) Osnovy paleontologii [Fundamentals of Paleontology] Vol. XI Agnatha, Pisces: 196-237. Izdatel'stvo 'Nauka', Moscow. [In Russian, translated 1967, Fundamentals of Paleontology, Vol. XI. Agnatha, Pisces. Israel Program for Scientific Translations, Jerusalem.]

Glickman L.S. 1980. Evolyutsiya melovykh i kajnozoyskikh lamnoidnyh akul [Evolution of Cretaceous and Cenozoic lamnoid sharks]. Nauka, Moscow. [In Russian.] [Not seen.]

Glickman L.S., Mertiniene R.A. \& Nessov L.A. 1987. Vertebrates. In: Glickman L.S., Mertiniene R.A., Nessov L.A., Rozhdestvensky A.K., Khosatzky L.I. \& Yakovlev V.N. (eds) Stratigrafiya SSSR, Melovaya sistema, Polutom 2: 252-262. Nedra, Moscow. [In Russian.]

Glinskiy V. 2018. Phylogenetic relationships of psammosteid heterostracans (Pteraspidiformes), Devonian jawless vertebrates. Biological Communications 62 (4) [for 2017]: 219-243.

https://doi.org/10.21638/11701/spbu03.2017.402

González-Rodríguez K.A., Schultze H.-P. \& Arratia G. 2013. Miniature armored teleosts from the Albian-Cenomanian (Cretaceous) of Mexico. In: Arratia G., Schultze H.-P. \& Wilson M.V.H. (eds) Mesozoic Fishes 5, Global Diversity and Evolution: 457-487. Verlag Dr. Friedrich Pfeil, Munich.

Goodrich E.S. 1909. Vertebrata Craniata (First fascicle: cyclostomes and fishes). In: Lankester E.R. (ed.) A Treatise on Zoology Part IX: 1-518. Adam and Charles Black, London.

Available from https://www.biodiversitylibrary.org/item/99568 [accessed 17 Jul. 2018]. 
Goody P.C. 1969a. Sedenhorstia dayi (Hay), a new elopoid from the Cenomanian of Hajula in the Lebanon. American Museum Novitates (2358): 1-23.

Available from http://hdl.handle.net/2246/2568 [accessed 17 Jul. 2018].

Goody P.C. 1969b. The relationships of certain Upper Cretaceous teleosts with special reference to the myctophoids. Bulletin of the British Museum (Natural History) Geology, supplement 7: 1-255. Available from https://biodiversitylibrary.org/page/41379409 [accessed 17 Jul. 2018].

Gorizdro-Kulczycka Z. 1950. Dwudysżne ryby dewońskie Gór Swiętokrzyskich [Les Dipneustes dévoniens du Massif de Ste Croix]. Acta Geologica Polonica 1 (2): 53-105. [In Polish and in French.] Available from https://geojournals.pgi.gov.pl/agp/article/view/12924/11382 [accessed 17 Jul. 2018].

Gouet D. 1978. Arthrodires Dolichothoraci du Dévonien inférieur du Spitsberg: anatomie, phylogénie, environnement. Unpublished doctoral thesis, Université Paris VI, Paris (mimeographed).

Gouet D. 1984a. Placoderm interrelationships: a new interpretation, with a short review of placoderm classifications. Proceedings of the Linnean Society of New South Wales 107 (3): 211-243.

Available from https://biodiversitylibrary.org/page/34925525 [accessed 27 Aug. 2018].

Gouet D. 1984b. Les poissons placodermes du Spitsberg, Arthrodires Dolichothoraci de la formation de Wood Bay (Dévonien inférieur). Cahiers de Paléontologie, Section Vertébrés, CNRS, Paris.

Gowda S.S. 1967. On a new fossil fish known from an otolith from the South Indian Cenomanian. Journal of the Geological Society of India 8: 119-129.

Available from http://www.geosocindia.org/index.php/jgsi/article/view/56327/44039 [accessed 16 Sep. 2018].

Grande L. 1982. A revision of the fossil genus $\uparrow$ Diplomystus, with comments on the interrelationships of clupeomorph fishes. American Museum Novitates (1718): 1-34.

Available from http://hdl.handle.net/2246/5342 [accessed 17 Jul. 2018].

Grande L. 1987. Redescription of Hypsidoris farsonensis (Teleostei: Siluriformes), with a reassessment of its phylogenetic relationships. Journal of Vertebrate Paleontology 7 (1): 24-54.

Available from http://www.jstor.org/stable/4523119 [accessed 17 Jul. 2018].

Grande L. 1988. A well preserved paracanthopterygian fish (Teleostei) from freshwater Lower Paleocene deposits of Montana. Journal of Vertebrate Paleontology 8 (2): 117-130.

Available from http://www.jstor.org/stable/4523185 [accessed 17 Jul. 2018].

Grande L. 2010. An Empirical Synthetic Pattern Study of Gars (Lepisosteiformes) and Closely Related Species, Based Mostly on Skeletal Anatomy: the Resurrection of Holostei. Copeia Supplement 2A. American Society of Ichthyologists and Herpetologists, Lawrence, Kansas.

Available from http://www.jstor.org/stable/20787269 [accessed 17 Jul. 2018].

Grande L. \& Bemis W.E. 1991. Osteology and phylogenetic relationships of fossil and recent paddlefishes (Polyodontidae) with comments on the interrelationships of Acipenseriformes. Memoir of the Society of Vertebrate Paleontology 1: 1-121. https://doi.org/10.2307/3889328

Grande L. \& Bemis W.E. 1996. Interrelationships of Acipenseriformes, with Comments on "Chondrostei". In: Stiassny M.L.J., Parenti L.R. \& Johnson G.D. (eds) Interrelationships of Fishes: 85-115. Academic Press, San Diego.

Grande L. \& Bemis W.E. 1998. A comprehensive phylogenetic study of amiid fishes (Amiidae) based on comparative skeletal anatomy, an empirical search for interconnected patterns of natural history. Memoir of the Society of Vertebrate Paleontology 4: 1-690. https://doi.org/10.2307/3889331 
Grande L. \& Hilton E.J. 2006. An exquisitely preserved skeleton representing a primitive sturgeon from the Upper Cretaceous Judith River Formation of Montana (Acipenseriformes: Acipenseridae: n. gen. and sp.). Memoir of the Journal of Paleontology 65: 1-39.

Available from http://www.jstor.org/stable/4095815 [accessed 17 Jul. 2018].

Grande L. \& Hilton E.J. 2009. A replacement name for $\uparrow$ Psammorhynchus Grande \& Hilton, 2006 (Actinopterygii, Acipenseriformes, Acipenseridae). Journal of Paleontology 83 (2): 317-318. https://doi.org/10.1666/08-137.1

Grande L. \& Lundberg J.G. 1988. Revision and redescription of the genus $\uparrow$ Astephus (Siluriformes: Ictaluridae) with a discussion of its phylogenetic relationships. Journal of Vertebrate Paleontology 8 (2): 139-171. Available from http://www.jstor.org/stable/4523187 [accessed 17 Jul. 2018].

Greenwood P.H. \& Patterson C. 1967. A fossil osteoglossoid fish from Tanzania (E. Africa). Journal of the Linnean Society (Zoology) 47: 211-223. https://doi.org/10.1111/j.1096-3642.1967.tb01404.x

Gregory J.T. 1973. Bibliography of Fossil Vertebrates 1969-1972. Geological Society of America Memoirs 141: 1-386.

Griffith J. 1977. The Upper Triassic fishes from Polzberg bei Lunz, Austria. Zoological Journal of the Linnean Society 60 (1): 1-93. https://doi.org/10.1111/j.1096-3642.1977.tb00834.x

Griffith J. \& Patterson C. 1963. The structure and relationships of the Jurassic fish Ichthyokentema purbeckensis. Bulletin of the British Museum (Natural History), Geology 8 (1): 1-43.

Available from https://biodiversitylibrary.org/page/36370444 [accessed 27 Aug. 2018].

Grogan E.D. \& Lund R. 2000. Debeerius ellefseni (fam. nov., gen. nov., spec. nov.), an autodiastylic chondrichthyan from the Mississippian Bear Gulch Limestone of Montana (USA), the relationships of the Chondrichthyes, and comments on gnathostome evolution. Journal of Morphology 243 (3): 219-245. https://doi.org/10.1002/(SICI)1097-4687(200003)243:3\%3C219::AID-JMOR1\%3E3.0.CO;2-1

Grogan E.D. \& Lund R. 2008. A basal elasmobranch, Thrinacoselache gracia n. gen \& sp. (Thrinacodontidae, new family) from the Bear Gulch Limestone, Serpukhovian of Montana, USA. Journal of Vertebrate Paleontology 28 (4): 970-988. https://doi.org/10.1671/0272-4634-28.4.970

Grogan E.D. \& Lund R. 2015. Two new Actinopterygii (Vertebrata, Osteichthyes) with cosmine from the Bear Gulch Limestone (Heath Fm., Serpukhovian, Mississippian) of Montana USA. Proceedings of the Academy of Natural Sciences of Philadelphia 164: 111-132. https://doi.org/10.1635/053.164.0114

Gross W. 1930. Die Fische des mittleren Old Red Sudlivlands, Geologische und Palaeontologische Abhandelungen 22 (18): 123-156.

Gross W. 1932. Die Fische des Baltischen Devons. Palaeontographica (Abteilung A: PaläozoologieStratigraphie) 79 (1-2): 1-74.

Gross W. 1933. Die Wirbeltiere des rheinischen Devons. Abhandlungen der Preußischen geologischen Landesanstalt (N.F.) 154: 1-83.

Gross W. 1937. Die Wirbeltiere des rheinischen Devons, Teil II. Abhandlungen der Preußischen geologischen Landesanstalt (N.F.) 176: 1-83.

Gross W. 1940. Acanthodier und Placodermen aus den Heterostius-Schichten Estlands und Lettlands. Annales Societatis Rebus Naturae Investigandis in Universitate Tartuensi Constitutae 46: 12-98.

Gross W. 1965. Über die Placodermen-Gattungen Asterolepis und Tiaraspis aus dem Devon Belgiens und einen fraglichen Tiraspis-Rest aus dem Devon Spitzbergens. Bulletin de l'Institut royal des Sciences naturelles de Belgique (Sciences de la Terre) 41 (16): 1-19. 
Available from http://biblio.naturalsciences.be/rbins-publications/bulletin-of-the-royal-belgianinstitute-of-natural-sciences/41-1965/irscnb_p4087_rbins17568_41_bulletin-16.pdf/at_download/file [accessed 15 Sep. 2018].

Gross W. 1969. Lophosteus superbus Pander, ein Teleostome aus dem Silur Oesels. Lethaia 2 (1): 1547. https://doi.org/10.1111/j.1502-3931.1969.tb01249.x

Günther A. 1871. Description of Ceratodus, a genus of ganoid fishes, recently discovered in rivers of Queensland, Australia. Philosophical Transactions of the Royal Society of London 161: 511-571. [Possibly published in 1872?] Available from http://www.jstor.org/stable/109041 [accessed 17 Jul. 2018].

Günther A. 1880. An Introduction to the Study of Fishes. Adam and Charles Black, Edinburgh. https://doi.org/10.5962/bhl.title.109739

Hairapetian V., Ginter M. \& Yazdi M. 2008. Early Frasnian sharks from central Iran. Acta Geologica Polonica 58 (2): 173-179.

Available from https://geojournals.pgi.gov.pl/agp/article/view/10018/8548 [accessed 17 Jul. 2018].

Halstead L.B. 1993. Agnatha. In: Benton M.J. (ed.) The Fossil Record 2: 573-581. Chapman and Hall, London. [Author also known as Tarlo L.B.H.]

Hampe O. 2003. Revision of the Xenacanthida (Chondrichthyes: Elasmobranchii) from the Carboniferous of the British Isles. Transactions of the Royal Society of Edinburgh, Earth Sciences 93 (3): 191-237. https://doi.org/10.1017/S0263593300000419

Hansen M.C. 1985. Systematic relationships of petalodontiform chondrichthyans. In: Dutro Jr. J.T. \& Pfefferkorn H.W. (eds) Compte rendu, Neuvième Congrès international de stratigraphie et de géologie $d u$ Carbonifère 5: 523-541. Southern Illinois University Press, Carbondale, Illinois.

Harris J.E. 1951. Diademodus hydei, a new fossil shark from the Cleveland Shale. Proceedings of the Zoological Society of London 120 (4): 683-697. https://doi.org/10.1111/j.1096-3642.1951.tb00672.x

Harry R.R. 1953. Studies on the bathypelagic fishes of the family Paralepididae. 1. Survey of the genera. Pacific Science 7 (2): 219-249. Available from http://hdl.handle.net/10125/8728 [accessed 27 Jul. 2018].

Hasse J.C.F. 1878a. Die fossilen Wirbel, morphologische Studien. Morphologisches Jahrbuch 4: 214268. Available from https://biodiversitylibrary.org/page/13018138 [accessed 15 Sep. 2018].

Hasse J.C.F. 1878b. Das natürliche System der Elasmobranchier auf Grundlage des Baues und der Entwickelung der Wirbelsäule. Zoologischer Anzeiger 1 (7): 144-148; (8): 167-172.

Available from https://biodiversitylibrary.org/page/30123346 [accessed 15 Sep. 2018].

Hay O.P. 1899. On some changes in the names, generic and specific, of certain fossil fishes. The American Naturalist 33 (394): 783-792. Available from http://www.jstor.org/stable/2454274 [accessed 17 Jul. 2018].

Hay O.P. 1902. Bibliography and catalogue of the fossil Vertebrata of North America. Bulletin of the United States Geological Survey (179): 1-868. https://doi.org/10.5962/bhl.title.20094

Hay O.P. 1903. On a collection of Upper Cretaceous fishes from Mount Lebanon, Syria, with descriptions of four new genera and nineteen new species. Bulletin of the American Museum of Natural History 19 (10): 395-452. Available from http://hdl.handle.net/2246/1500 [accessed 17 Jul. 2018].

Hay O.P. 1929. Second Bibliography and Catalogue of the Fossil Vertebrata of North America Vol. I. Publication Nr. 390, Carnegie Institution of Washington, Washington. [Published August 1929.] Available from http://vertpaleo.org/Publications/Bibliography-of-Fossil-Vertebrates.aspx [accessed 15 Sep. 2018]. 
Heckel J.J. \& Kner R. 1857. Die Süsswasserfische der östreichischen Monarchie, mit Rücksicht auf die angränzenden Länder. Engelmann, Leipzig. https://doi.org/10.5962/bhl.title.8197

Heintz A. 1929. Die downtonischen und devonischen Vertebraten von Spitsbergen II: Acanthaspida. Skrifter om Svalbard og Ishavet (22): 1-79. [Printed 31 Jan. 1929.] Available from http://hdl.handle.net/11250/173607 [accessed 17 Jul. 2018].

Heintz A. 1932. The structure of Dinichthys. A contribution to our knowledge of the Arthrodira. In: Gudger E.W. (ed.) The Bashford Dean Memorial Volume Archaic Fishes Part I: 115-224.

Available from https://biodiversitylibrary.org/page/6285867 [accessed 15 Sep. 2018].

Heintz A. 1934. Revision of the Estonian Arthrodira. Part I. Family Homostiidae Jaekel. Archiv für die Naturkunde Estlands (series 1) 10 (4): 1-114. Available from http://dspace.ut.ee/bitstream/ handle/10062/46600/est_a_1682_1_10_4_ocr.pdf? sequence=1\&isAllowed=y [accessed 15 Sep. 2018].

Heintz A. 1937. Die downtonischen und devonischen Vertebraten von Spitzbergen VI: LunaspisArten aus dem Devon Spitzbergens. Skrifter om Svalbard og Ishavet (72): 1-23. Available from http://hdl.handle.net/11250/173609 [accessed 17 Jul. 2018].

Heintz A. 1940. Cephalaspida from Downtonian of Norway. Skrifter Utgitt av det Norske VidenskapsAkademi i Oslo. Matematisk-Naturvidenskapelig Klasse 1939 (5): 1-119.

Heintz A. 1949. Cephalaspider fra Ringerike. Naturen 73: 321-340. [In Norwegian.]

Heintz A. 1962. Les organes olfactifs des Heterostraci. [The development of the rostro-oral region in the Heterostraci]. Problèmes actuels de paléontologie: évolution des vertébrés. Colloques Internationaux du Centre nationale de la Recherche scientifique 104: 13-29.

Heintz A. 1968. The pteraspid Lyktaspis n. g. from the Devonian of Vestspitsbergen. In: Ørvig T. (ed.) Current problems of lower vertebrate phylogeny. Proceedings of the $4^{\text {th }}$ Nobel Symposium 1967, Stockholm: 73-80. Almqvist and Wiksell, Stockholm.

Heintz N. 1962. Gigantaspis, a new genus of fam. Pteraspididae from Spitsbergen. Norsk Polarinstitutt Arbok 1960: 22-27. Available from http://hdl.handle.net/11250/172773 [accessed 27 Aug. 2018].

Herman J. 1977. Les sélaciens des terrains néocrétacés and paléocènes de Belgique et des contrées limitrophes. Eléments d'une biostratigraphie intercontinentale. Mémoires pour servir à l'explication des Cartes géologiques et minières de la Belgique (Service Géologique de Belgique) 15, Belgian Geological Survey, Brussels. Available from http://www.vliz.be/imisdocs/publications/ocrd/251574.pdf [accessed 15 Sep. 2018].

Herman J. 1979. Réflexions sur la systématique des Galeoidei et sur les affinités du genre Cetorhinus à l'occasion de la découverte d'éléments de la denture d'un exemplaire fossile dans les sables du Kattendijk à Kallo (Pliocène Inférieur, Belgique). Annales de la Société géologique de Belgique 102: 357-377. Availabe from https://popups.uliege.be/0037-9395/index.php?id=4361\&file=1\&pid=4357 [accessed 17 Jul. 2018].

Herman J. \& van den Eeckhaut G. 2010. Inventaire systématique des Invertebrata, Vertebrata, Plantae et Fungi des Sables de Bruxelles à Zaventem. Géominpal Belgica (découvertes géologiques, minéralogiques et paléontologiques en Belgique) (1): 32-64.

Herman J. \& van Waes H. 2012a. Observations diverses et déductions concernant l'évolution et la systématique de quelques Euselachii, Neoselachii et Batoidei (Pisces - Elasmobranchii), actuels et fossiles. (données complémentaires). Géominpal Belgica (découvertes géologiques, minéralogiques et paléontologiques en Belgique) (2): 1-11.

Herman J. \& van Waes H. 2012b. Observations diverses et déductions concernant l'évolution et la systématique de quelques Euselachii, Neoselachii et Batoidei (Pisces - Elasmobranchii), actuels et 
fossiles (fin). Géominpal Belgica (découvertes géologiques, minéralogiques et paléontologiques en Belgique) (2): 1-13.

Herman J. \& van Waes H. 2012c. Observations concerning the evolution and the parasystematic of all the living and fossil Heterodontiformes. Géominpal Belgica (découvertes géologiques, minéralogiques et paléontologiques en Belgique) (3): 1-15.

Herman J. \& van Waes H. 2012d. Observations concerning the evolution and the parasystematic of all the living and fossil Scyliorhiniformes and Carcharhiniformes and suggestions concerning the possible origins of the Batoidei. Géominpal Belgica (découvertes géologiques, minéralogiques et paléontologiques en Belgique) (4): 1-97.

Herman J. \& van Waes H. 2014. Observations concerning the evolution and the parasystematics of all the living and fossil Chlamydoselachiformes, Squatiniformes, Orectolobiformes, and Pristiophoriformes, based on both biological and odontological data. Suggestion of a possible origin of the order Pristiophoriformes, of the order Ganopristiformes and a global synthesis of the previous systematics proposals. Géominpal Belgica (découvertes géologiques, minéralogiques et paléontologiques en Belgique) (6): 1-347.

Heyler D. 1969. Vertébrés de l'Autunien de France. Cahiers de paléontologie. Éditions du Centre National de la Recherche Scientifique, Paris.

Heyler D. 1977. Aeduelliformes de l'Autunien français et actinoptérygiens du Ladinien (?) de Brookvale (Australie). Bulletin de la Société d'Histoire naturelle d'Autun 83: 9-19.

Available from https:/gallica.bnf.fr/ark:/12148/bpt6k97348826/f11.image [accessed 16 Sep. 2018].

Heyler D. \& Poplin C. 1983. Actinoptérygiens du Stéphanien de Montceau-les-Mines (Saône-et-Loire, France). Palaeovertebrata 13 (3): 33-50.

Available from http://palaeovertebrata.com/Articles/sendFile/104/published_article [accessed 17 Jul. 2018].

Heyler D. \& Poplin C. 1990. Systematics and relationships among the Xenacanthiformes (Pisces, Chondrichthyes) in the light of Carboniferous and Permian French material. Acta Musei Reginaehradecensis Series A, Scientiae naturales 22 [for 1989]: 69-78.

Hilton E.J., Grande L. \& Bemis W.E. 2011. Skeletal Anatomy of the Shortnose Sturgeon, Acipenser brevirostrum Lesueur, 1818, and the Systematics of Sturgeons (Acipenseriformes, Acipenseridae). Fieldiana Life and Earth Sciences 3, Field Museum, Chicago. https://doi.org/10.3158/2158-5520-3.1.1

Hoernes R. 1884. Elemente der Palaeontologie (Palaeozoologie). Veit \& Comp., Leipzig. https://doi.org/10.5962/bhl.title.14950

Hubbs C.L. 1952. A contribution to the classification of the blennioid fishes of the family Clinidae, with a partial revision of eastern Pacific forms. Stanford Ichthyological Bulletin 4 (2): 41-165.

Huddleston R.W. 1981. Bernardichthys zorraquinosi, a new genus and species of salmoniform fish from the late Cretaceous of Oregon. Proceedings of the Biological Society of Washington 94 (1): 37-42. Available from https://www.biodiversitylibrary.org/page/34607888 [accessed 17 Jul. 2018].

Hughes L.C., Ortí G., Huang Y., Sun Y., Baldwin C.C., Thompson A.W., Arcila D., Betancur-R. D., Li C., Becker L., Bellora N., Zhao X., Li X., Wang M., Fang C., Xie B., Zhou Z., Huang H., Chen S., Venkatesh B. \& Shi Q. 2018. Comprehensive phylogeny of ray-finned fishes (Actinopterygii) based on transcriptomic and genomic data. Proceedings of the National Academy of Sciences 115 (24): 62496254. https://doi.org/10.1073/pnas.1719358115

Hutchinson P. 1973. A revision of the redfieldiiform and perleidiform fishes from the Triassic of Bekker's Kraal (South Africa) and Brookvale (New South Wales). Bulletin of the British Museum (Natural 
History), Geology 22 (3): 235-354. Available from https://www.biodiversitylibrary.org/page/36949634 [accessed 17 Jul. 2018].

Huxley T.H. 1861. Preliminary essay upon the systematic arrangement of the fishes of the Devonian epoch. Memoirs of the Geological Survey of the United Kingdom (figures and descriptions illustrative of British organic remains) Decade X: 1-49. Available from https://biodiversitylibrary.org/page/45186111 [accessed 17 Jul. 2018].

ICZN 2018. International Commision on Zoological Nomenclature.

Available from http://iczn.org/ and http://iczn.org/code [accessed 27 Aug. 2018].

Ishihara H., Treloar M., Bor P.H.F., Senou H. \& Jeong C.H. 2012. The comparative morphology of skate egg capsules (Chondrichthyes: Elasmobranchii: Rajiformes). Bulletin of the Kanagawa Prefectural Museum (Natural Science) No. 41: 9-25.

Available from http://nh.kanagawa-museum.jp/files/data/pdf/bulletin/41/bull41_09-25_ishihara_h.pdf [accessed 17 Jul. 2018].

Jaekel O.M.J. 1890. Über fossile Ichthyodorulithen (Über Flossenstacheln oder Ichthyodorulithen im Allgemeinen). Sitzungsberichte der Gesellschaft naturforschender Freunde zu Berlin 1890 (7): 117131. Available from https://biodiversitylibrary.org/page/8791075 [accessed 28 Aug. 2018].

Jaekel O.M.J. 1898. Über die verschiedenen Rochentypen. Sitzungsberichte der Gesellschaft naturforschender Freunde zu Berlin 1898 (5): 44-53. [Sometimes dated to 1899.]

Available from https://biodiversitylibrary.org/page/8790763 [accessed 28 Aug. 2018].

Jaekel O.M.J. 1903. Die Organisation und systematische Stellung der Asterolepiden. Zeitschrift Monatsberichte der Deutschen geologischen Gesellschaft 55: 41-60.

Available from https://biodiversitylibrary.org/page/43918061 [accessed 28 Aug. 2018].

Jaekel O.M.J. 1907. Über Pholidosteus nov. gen., die Mundbildung und die Körperform der Placodermen. Sitzungsberichte der Gesellschaft naturforschender Freunde zu Berlin 1907 (6): 170-186.

Available from https://biodiversitylibrary.org/page/8795896 [accessed 28 Aug. 2018].

Jaekel O.M.J. 1911. Die Wirbeltiere, eine Übersicht über die fossilen und lebenden Formen. Gebrüder Borntraeger, Berlin. https://doi.org/10.5962/bhl.title.119340

Jaekel O.M.J. 1919. Die Mundbildung der Placodermen. Sitzungsberichte der Gesellschaft naturforschender Freunde zu Berlin 1919 (3): 73-110.

Available from https://biodiversitylibrary.org/page/10289172 [accessed 28 Aug. 2018].

Janvier Ph. 1981. Norselaspis glacialis n. g., n. sp. et les relations phylogénétiques entre les Kiaeraspidiens (Osteostraci) du Dévonien inférieur du Spitsberg. Palaeovertebrata 11 (2-3): 19-131. Available from http://palaeovertebrata.com/Articles/sendFile/89/published_article [accessed 17 Jul. 2018].

Jarvik E. 1985. Devonian osteolepiform fishes from East Greenland. Meddelelser om Grønland, Geoscience 13: 1-51.

Jerzmańska A. 1969. Ichtyofaune des couches à ménilite (Flysch des Karpathes). Acta Palaeontologica Polonica 13 (3): 379-488. Available from https://www.app.pan.pl/archive/published/app13/app13-379.pdf [accessed 17 Jul. 2018].

Jessen H.L. 1980. Lower Devonian Porolepiformes from the Canadian Arctic with special reference to Powichthys thorsteinssoni Jessen. Palaeontographica (Abteilung A: Paläozoologie-Stratigraphie) 167 (4-6): 180-214.

Jia L.-T. 2008. [A new species of antiarchs from Upper Devonian of Qingtongxia, Ningxia and its paleobiogeographic significance]. Unpublished master thesis, Institute of Vertebrate Paleontology and Paleoanthropology, Chinese Academy of Sciences, not available. [In Chinese, with English abstract.] 
Jordan D.S. 1905. A Guide to the Study of Fishes Volume 2. Henry Holt and Company, New York. Available from https://www.biodiversitylibrary.org/item/118481 [accessed 17 Jul. 2018].

Jordan D.S. 1919. Fossil fishes of southern California I. Fossil fishes from the Soledad deposits of southern California. Leland Stanford Junior University Publications, University series: 3-12. https://doi.org/10.5962/bhl.title.28466

Jordan D.S. 1923. A Classification of Fishes including Families and Genera as Far as Known. Stanford University Publications, University series, Biological Sciences 3 (2), Stanford University, Stanford. [January.]

Jordan D.S. 1925a. A collection of fossil fishes in the University of Kansas, from the Niobrara Formation of the Cretaceous. The Kansas University Science Bulletin 15 (2): 219-245. [For December 1924.] Available from https://biodiversitylibrary.org/page/15421807 [accessed 16 Sep. 2018].

Jordan D.S. 1925b. The fossil fishes of the Miocene of southern California. Contribution no. VIII. [With sections: A. Species from the diatom beds at Lompoc; B. Fossil fishes from Los Angeles County, California; C. Fishes from a quarry in Monterey County near San Miguel.]. Stanford University Publications, University Series, Biological Sciences 4 (1): 1-51. https://doi.org/10.5962/bhl.title.28465

Jordan D.S. \& Gilbert J.Z. 1919. Fossil fishes of southern California II. Fossil fishes of the Miocene (Monterey) formations of southern California. Leland Stanford Junior University publications, University Series: 13-60. https://doi.org/10.5962/bhl.title.28466

Jordan D.S. \& Gilbert J.Z. 1920. Fossil fishes of diatom beds of Lompoc, California. Leland Stanford Junior University Publications, University Series (42): 1-44. https://doi.org/10.5962/bhl.title.26061

Jörg E. 1969. Eine Fischfauna aus dem Oberen Buntsandstein (Unter-Trias) von Karlsruhe-Durlach (Nordbaden). Beiträge zur naturkundlichen Forschung in Südwestdeutschland 28: 87-102.

Karatajūtè-Talimaa V.N. 1968. Noviye telodonti, heterostraki i artrodiri iz Chortkovskogo Horizonta Podolii [New thelodonts, heterostracans and arthrodires from the Chortkov Stage of Podolia]. In: Obruchev V.D. (ed.) Ocherki po filogenii i sistematike iskopayemykh ryb i beschelyustnykh [Outlines on the Phylogeny and Systematics of the Fossil Fishes and Agnathans]: 33-42. Nauka, Moscow. [In Russian.]

Karatajūtè-Talimaa V.N. 1970. Ikhtiofauna Dauntona Litvi, Estonii i severnogo Timana. Paleontologiya i Stratigrafiya Pribaltiki i Byelorussiai 2: 33-66. [In Russian.]

Karatajūtè-Talimaa V.N. 1978. Telodonty silura i devona SSSR i Shpitsbergena [Silurian and Devonian thelodonts of the U.S.S.R. and Spitsbergen]. Mosklas, Vilnius. [In Russian.]

Karatajūtè-Talimaa V.N. 1989. [Skalviaspis narbutasi gen. et sp. nov., a new representative of the Pteraspidida (Heterostraci) from the East Baltic Lower Devonian]. Geologija (Vilnius) 10: 79-93. [In Russian.]

Karatajūtè-Talimaa V.N. 1997a. Lugalepis - a new genus of elasmobranchs from Devonian of the western part of the Main Devonian Field. Geologija (Vilnius) 21: 24-29.

Karatajūtė-Talimaa V.N. 1997b. Taxonomy of loganiid thelodonts. Modern Geology 21 (1-2): 1-15.

Karatajūtè-Talimaa V.N. \& Märss T. 2004. [Agnathans and early fishes: subclass Thelodonti (thelodonts)]. In: Novitskaya L.I. \& Afanassieva O.B. (eds) Iskopayemyye pozvonochnyye Rossii i sopredel'nykh stran. Beschelyustnyye i drevniye ryby. Spravochnik dlya paleontologov, biologov i geologov [Fossil Vertebrates of Russia and Adjacent Countries. The Reference Book for Paleontologists, Biologists and Geologists]: 12-68. GEOS, Moscow. [In Russian.] 
Karatajute-Talimaa V.N., Novitskaya L.I., Rozman K.S. \& Sodov Z. 1990. Mongolepis - novyj rod elasmobranchij iz nizhnego silura Mongolii [Mongolepis - a new Lower Silurian elasmobranch genus from Mongolia]. Paleontologicheskii Zhurnal 1990 (1): 76-86. [In Russian.]

Karatajūtè-Talimaa V.N. \& Smith M.M. 2003. Early acanthodians from the Lower Silurian of Asia. Transactions of the Royal Society of Edinburgh, Earth Sciences 93 (3): 277-299.

https://doi.org/10.1017/S0263593300000444

Karatajūtè-Talimaa V.N. \& Smith M.M. 2004. Tesakoviaspis concentrica: microskeletal remains of a new order of vertebrate from the Upper Ordovician and Lower Silurian of Siberia. In: Arratia G., Wilson M.V.H. \& Cloutier R. (eds) Recent Advances in the Origin and Early Radiation of Vertebrates: 53-64. Verlag Dr. Friedrich Pfeil, Munich.

Karpinsky A.P. 1911. [Notes sur l'Helicoprion et les autres Édestides / Notes on Helicoprion and the other edestids]. Izviestiia Imperatorskoi akademii nauk / Bulletin de l'Académie Impériale des Sciences de St.-Pétersbourg (série VI) 5 (16): 1105-1122. [In Russian.]

Available from https://biodiversitylibrary.org/page/29688963 [accessed 16 Sep. 2018].

Kazantseva A.A. 1968. Paleoniskidy bystr'anskoy svity Minussinskikh kotlovin [The palaeoniscids of the Bystr'anskaya Formation of the Minussinsk Depression]. In: Obruchev V.D. (ed.) Ocherki po filogenii i sistematike iskopayemykh ryb i beschelyustnykh [Outlines on the Phylogeny and Systematics of the Fossil Fishes and Agnathans]: 87-112. Nauka, Moscow. [In Russian.]

Kazantseva A.A. 1971. K sistematike Palaeonisciformes [On the systematics of Palaeonisciformes]. Trudy Paleontologicheskogo Instituta Akademii Nauk SSSR 130: 160-167. [In Russian.]

Kazantseva-Selezneva A.A. 1977. K sisteme i filogenii otryada Palaeonisciformes [On the systematics and phylogeny of the order Palaeonisciformes]. In: Menner V.V. (ed.) Ocherki po filogenii i sistematike iskopayemykh ryb $i$ beschelyustnykh [Outlines on the phylogeny and systematics of fossil fishes and agnathans]: 98-115. Paleontologicheskogo Instituta Akademii Nauka, Moscow. [In Russian.]

Kazantseva-Selezneva A.A. 1981. Filogeniya nizshikh lucheperykh [Phylogeny of the lower actinopterygians]. Voprosy Ikhtiologii 21 (4): 579-594. [In Russian, English translation in 1982: Journal of Ichthyology: 1-16.]

Kiaer J. 1924. The Downtonian fauna of Norway. 1. Anaspida, with a geological introduction. Skrifter utgit av Videnskapsselskapet i Kristiania, I Matematisk-naturvidenskabelig Klasse 1924 (6): 1-163.

Kiaer J. 1930. Ctenaspis a new genus of cyathaspidian fishes: a preliminary report. Skrifter om Svalbard og Ishavet (33): 1-7. Available from http://hdl.handle.net/11250/173724 [accessed 17 Jul. 2018].

Kiaer J. 1932. The Downtonian and Devonian vertebrates of Spitsbergen. IV. Suborder Cyathaspida. Skrifter om Svalbard og Ishavet (52): 1-26. Available from http://hdl.handle.net/11250/173597 [accessed 27 Jul. 2018].

Kiaer J. \& Heintz A. 1935. The Downtonian and Devonian vertebrates of Spitsbergen, V. Suborder Cyathaspida Part I: tribe Poraspidei, family Poraspidae Kiaer. Skrifter om Svalbard og Ishavet (40): 1-138. Available from http://hdl.handle.net/11250/173666 [accessed 17 Jul. 2018].

Kirkland J.I. \& Aguillón-Martínez M.C. 2002. Schizorhiza, a unique sawfish paradigm from the Difunta Group, Coahuila, Mexico. Revista Mexicana de Ciencias Geológicas 19 (1): 16-24. Available from http://satori.geociencias.unam.mx/19-1/(2)Kirkland.pdf [accessed 17 Jul. 2018].

Klug S. \& Kriwet J. 2012. Node age estimations and the origin of angel sharks, Squatiniformes (Neoselachii, Squalomorphii). Journal of Systematic Palaeontology 11 (1) [for 2013]: 91-110. [Published online 30 Nov. 2012.] https://doi.org/10.1080/14772019.2012.674066 
Kner R. 1867. Neuer Beitrag zur Kenntniß der fossilen Fische von Comen bei Görz. Sitzungsberichte der Akademie der Wissenschaften mathematisch-naturwissenschaftliche Klasse 56: 171-200.

Available from https://biodiversitylibrary.org/page/6476857 [accessed 28 Aug. 2018].

Koch L. 1931. Carboniferous and Triassic stratigraphy of East Greenland. Meddelelser om Grønland 83 (2): 1-99.

Koninck L.-G. de 1878. Faune de calcaire carbonifère de la Belgique. Première partie Poissons et Genre Nautile. Annales du Musée royal d'Histoire naturelle de Belgique 2, Musée royal d'Histoire naturelle de Belgique, Brussels.

Available from https://biodiversitylibrary.org/page/56041931 [accessed 28 Aug. 2018].

Kossovoy L.S. \& Obruchev D.V. 1962. [The Lower Devonian in north Timan], Doklady Akademii Nauk SSSR / Comptes rendus de l'Académie des sciences de l'URSS (N.S.) 147 (5): 1147-1150. [In Russian, first author also seen as Kossovoi; English translation: 106-108.]

Kriwet J. 1999. Pycnodont fishes (Neopterygii, $†$ Pycnodontiformes) from the Lower Cretaceous of Uña (E-Spain) with comments on branchial teeth in pycnodontid fishes. In: Arratia G. \& Schultze H.-P. (eds) Mesozoic Fishes 2, Systematics and Fossil Record, Proceedings of the International Meeting Buckow 1997: 215-238. Verlag Dr. F. Pfeil, Munich.

Kriwet J. \& Klug S. 2011. A new Jurassic cow shark (Chondrichthyes, Hexanchiformes) with comments on Jurassic hexanchiform systematics. Swiss Journal of Geosciences 104 (Supplement 1): S107-S114. https://doi.org/10.1007/s00015-011-0075-z

Kriwet J. \& Klug S. 2016. Crassodontidanidae, a replacement name for Crassonotidae Kriwet and Klug, 2011 (Chondrichthyes, Hexanchiformes). Journal of Vertebrate Paleontology 36 (4): e1119698. https://doi.org/10.1080/02724634.2016.1119698

Kriwet J., Klug S., Canudo J.I. \& Cuenca-Bescós G. 2008. A new Early Cretaceous lamniform shark (Chondrichthyes, Neoselachii). Zoological Journal of the Linnean Society 154 (2): 278-290. https://doi.org/10.1111/j.1096-3642.2008.00410.x

Kriwet J., Nunn E.V. \& Klug S. 2009. Neoselachians (Chondrichthyes, Elasmobranchii) from the Lower and lower Upper Cretaceous of north-eastern Spain. Zoological Journal of the Linnean Society 155 (2): 316-347. https://doi.org/10.1111/j.1096-3642.2008.00439.x

Krupina N.I. 2004. [Agnathans and early fishes: subclass Dipnoi (dipnoans)]. In: Novitskaya L.I. \& Afanassieva O.B. (eds) Iskopayemyye pozvonochnyye Rossii i sopredel'nykh stran. Beschelyustnyye $i$ drevniye ryby. Spravochnik dlya paleontologov, biologov i geologov [Fossil Vertebrates of Russia and Adjacent Countries. The Reference Book for Paleontologists, Biologists and Geologists]: 373-413. GEOS, Moscow. [In Russian.]

Lambers P.H. 1996. A redescription of the coelacanth Macropoma willemoesii Vetter from the lithographic limestone of Solnhofen (Upper Jurassic, Bavaria). In: Arratia G. \& Viohl G. (eds) Mesozoic Fishes Systematics and Paleoecology, Proceedings of the International Meeting Eichstätt 1993: 395407. Verlag Dr. Friedrich Pfeil, Munich.

Landemaine O. 1991. Sélaciens nouveaux du Crétacé Supérieur du Sud-Ouest de la France, quelques apports à la systématique des élasmobranches. Société amicale des Géologues amateurs (National Museum of Natural History, Paris) 1: 1-45.

Lane J. \& Ebert M. 2015. A taxonomic reassessment of Ophiopsis (Halecomorphi, Ionoscopiformes), with a revision of Upper Jurassic species from the Solnhofen Archipelago, and a new genus of Ophiopsidae. Journal of Vertebrate Paleontology 35 (1): e948546. https://doi.org/10.1080/02724634.2014.883238

Le Danois E. \& Le Danois Y. 1964. L'ordre des Scombres. Mémoires de l'IFAN 68: 153-192. 
Lebedev O.A. 1995. Middle Famennian (Upper Devonian) chondrichthyans and sarcopterygians from Oryol region; Central Russia. Geobios 28 (Supplement 2): 361-368.

https://doi.org/10.1016/S0016-6995(95)80139-1

Lebedev O.A. 2008. Systematics and dental system reconstruction of the durophagous chondrichthyan Lagarodus Jaekel, 1898. Acta Geologica Polonica 58 (2): 199-204. Available from https://geojournals.pgi.gov.pl/agp/article/view/10001/8531 [accessed 27 Jul. 2018].

Lehman J.-P. 1952. Étude complémentaire des poissons de l'Eotrias de Madagascar. Kungliga Svenska Vetenskapsakademiens Handlingar (series 4) 2 (6): 1-201.

Lehman J.-P. 1966. Les Actinoptérygiens, Crossoptérygiens, Dipneustes. In: Piveteau J. (ed.) Traité de Paléontologie Tome 4 Fascicule 3: 398-420. Masson \& Cie, Paris.

Leidner A. \& Delsate D. 2000. The new family Welcommiidae Leidner and Delsate, 2000. A working theory and preliminary results. Abstract book of the $5^{\text {th }}$ EWVP, Karlsruhe. [Unavailable publication.]

Leidy J. 1857. Remarks on certain extinct species of fishes. Proceedings of the Academy of Natural Sciences of Philadelphia 8: 301-302. Available from http://www.jstor.org/stable/4059166 [accessed 17 Jul. 2018].

Liu H.-T. \& Zhou J.J. 1965. A new sturgeon from the upper Jurassic of Liaoning, North China. Vertebrata PalAsiatica 9 (3): 237-247. [In Chinese, with English summary.] Available from http://www.ivpp.cas.cn/cbw/gjzdwxb/xbwzxz/200912/P020091223542408620405.pdf [accessed 17 Jul. 2018].

Liu J., Chang M.-M., Wilson M.V.H. \& Murray A.M. 2015. A new family of Cypriniformes (Teleostei, Ostariophysi) based on a redescription of $\dagger$ Jianghanichthys hubeiensis (Lei, 1977) from the Eocene Yangxi Formation of China. Journal of Vertebrate Paleontology 35 (6): e1004073.

https://doi.org/10.1080/02724634.2015.1004073

Liu S.-F. 1986. Fossil eugaleaspid from Guangxi. Vertebrata PalAsiatica 24 (1): 1-9. [In Chinese, with English summary.]

Available from http://www.ivpp.cas.cn/cbw/gjzdwxb/xbwzxz/200903/W020090813373830261955.pdf [accessed 17 Jul. 2018].

Liu T.-S. \& Pan K. 1958. Devonian fishes from Wutung Series near Nanking, China. Palaeontologia Sinica (new series C) 15 (141): 1-41.

Liu X.-T., Ma F.-C. \& Liu Z.-C. 1982. [Pisces]. In: Geological Bureau of Nei Mongol Autonomous Region (eds) The Mesozoic Stratigraphy and Paleontology of Guyang Coalbearing Basin, Nei Mongol, China: 101-122. Geological Publishing House, Beijing. [In Chinese.]

Liu X.-T. \& Wang N.-Z. 1978. [The Upper Permian fish-fauna of Dzungaria Basin, Sinkiang]. Memoirs of Institute of Vertebrate Palaeontology and Palaeoanthropology, Academia Sinica (13): 1-18. [In Chinese, authors as Liu H.-T. and Wang N.-C.]

Liu Y.-H. 1965. New Devonian agnathans of Yunnan. Vertebrata PalAsiatica 9 (2): 125-140. [In Chinese, with English summary.]

Available from http://www.ivpp.cas.cn/cbw/gjzdwxb/xbwzxz/200912/P020091221561091002070.pdf [accessed 17 Jul. 2018].

Liu Y.-H. 1973. On the new forms of Polybranchiaspiformes and Petalichthyida from Devonian of south-west China. Vertebrata PalAsiatica 11 (2): 132-143. [In Chinese.]

Available from http://www.ivpp.cas.cn/cbw/gjzdwxb/xbwzxz/200905/W020090813377786124936.pdf [accessed 17 Jul. 2018]. 
Liu Y.-H. 1975. Lower Devonian agnathans of Yunnan and Sichuan. Vertebrata PalAsiatica 13 (4): 202-216. [In Chinese, with English summary.]

Available from http://www.ivpp.cas.cn/cbw/gjzdwxb/xbwzxz/200905/W020090813377232796719.pdf [accessed 17 Jul. 2018].

Liu Y.-H. 1980. A nomenclatural note on Eugaleaspis for Galeaspis Liu, 1965; Eugaleaspidae for Galeaspidae Liu, 1965; Eugaleaspiformes for Galeaspiformes. Vertebrata PalAsiatica 18 (3): 256-257. [In Chinese, with English summary.]

Liu Y.-H. 1991. On a new petalichthyid, Eurycaraspis incilis gen. et sp. nov., from the Middle Devonian of Zhanyi, Yunnan. In: Chang M.-M., Liu Y.-H. \& Zhang G.-R. (eds) Early Vertebrates and related problems of evolutionary biology: 139-177. Science Press, Beijing.

Long J.A. 1983a. New bothriolepid fishes from the Late Devonian of Victoria, Australia. Palaeontology 26 (2): 295-320.

Available from https://www.palass.org/sites/default/files/media/publications/palaeontology/volume_26/ vol26_part2_pp295-320.pdf [accessed 17 Jul. 2018].

Long J.A. 1983b. A new diplacanthoid acanthodian from the Late Devonian of Victoria, Australia. Memoirs of the Association of Australasian Palaeontologists 1: 51-66.

Long J.A. 1992. Gogodipterus paddyensis (Miles), gen. nov., a new chirodipterid lungfish from the late Devonian Gogo formation, Western Australia. The Beagle, Records of the Museums and Art Galleries of the Northern Territory 9 (1): 11-20.

Long J.A. 2011. The Rise of Fishes. Johns Hopkins University Press, Baltimore, USA.

Long J.A., Choo B. \& Young G.C. 2008. A new basal actinopterygian fish from the Middle Devonian Aztec Siltstone of Antarctica. Antarctic Science 20 (4): 393-412.

https://doi.org/10.1017/S0954102008001144

Long J.A., Mark-Kurik E. \& Young G.C. 2014. Taxonomic revision of buchanosteoid placoderms (Arthrodira) from the Early Devonian of south-eastern Australia and Arctic Russia. Australian Journal of Zoology 62 (1): 26-43. https://doi.org/10.1071/ZO13081

Loomis F.B. 1900. Die Anatomie und die Verwandtschaft der Ganoid- und Knochen-Fische aus der Kreide-Formation von Kansas, U.S.A. Palaeontographica 46: 213-283.

Available from https://biodiversitylibrary.org/page/33297714 [accessed 16 Sep. 2018].

López-Arbarello A. 2012. Phylogenetic interrelationships of ginglymodian fishes (Actinopterygii: Neopterygii). PLoS One 7 (7): 1-44 [e39370]. https://doi.org/10.1371/journal.pone.0039370

López-Arbarello A. \& Zavattieri A.M. 2008. Systematic revision of Pseudobeaconia Bordas, 1944, and Mendocinichthys Whitley, 1953 (Actinopterygii: 'Perleidiformes') from the Triassic of Argentina. Palaeontology 51 (5): 1025-1052. http://doi.org/10.1111/j.1475-4983.2008.00806.x

Lu L.-W. \& Chen X.-Y. 2010. A review on the study of Carboniferous-Permian vertebrates in China. In: Dong W. (ed.) Proceedings of the twelfth annual meeting of the Chinese Society of Vertebrate Paleontology: 1-12. China Ocean Press, Beijing. [In Chinese, with English summary.] [Not seen.]

Lund R. 1968. The Pholidophoriformes and the Origin of the Teleosts. Unpublished dissertation, Columbia University, NewYork, USA.

Lund R. 1974. Stethacanthus altonensis (Elasmobranchii) from the Bear Gulch limestone of Montana. Annals of Carnegie Museum 45 (8): 161-178.

Lund R. 1977. Echinochimaera meltoni new genus and species (Chimaeriformes), from the Mississippian of Montana. Annals of Carnegie Museum 46 (13): 195-221. 
Lund R. 1989. New petalodonts (Chondrichthyes) from the Upper Mississippian Bear Gulch limestone (Namurian $\mathrm{E}_{2} \mathrm{~b}$ ) of Montana. Journal of Vertebrate Paleontology 9 (3): 350-368. Available from http://www.jstor.org/stable/4523270 [accessed 17 Jul. 2018].

Lund R. 2000. The new Actinopterygian order Guildayichthyiformes from the Lower Carboniferous of Montana (USA). Geodiversitas 22 (2): 171-206.

Available from http://sciencepress.mnhn.fr/sites/default/files/articles/pdf/g2000n2a2.pdf [accessed 17 Jul. 2018].

Lund R. \& Grogan E.D. 1997. Cochliodonts from the Mississippian Bear Gulch Limestone Heath Formation; Big Snowy Group, Chesterian) of Montana and the relationships of the Holocephali. In: Wolberg D.L., Stump E. \& Rosenberg G.D. (eds) Proceedings of the Dinofest International Symposium: 477-492. The Academy of Natural Sciences, Philadelphia.

Lund R. \& Grogan E.D. 2004a. Two tenaculum-bearing Holocephalimorpha (Chondrichthyes) from the Bear Gulch Limestone (Chesterian, Serpukhovian) of Montana, USA. In: Arratia G., Wilson M.V.H. \& Cloutier R. (eds) Recent Advances in the Origin and Early Radiation of Vertebrates: 177-187. Verlag Dr. Friedrich Pfeil, Munich.

Lund R. \& Grogan E.D. 2004b. Five new euchondrocephalan Chondrichthyes from the Bear Gulch Limestone (Serpukhovian, Namurian E2b) of Montana, USA. In: Arratia G., Wilson M.V.H. \& Cloutier R. (eds) Recent Advances in the Origin and Early Radiation of Vertebrates: 505-531. Verlag Dr. Friedrich Pfeil, Munich.

Lund R. \& Lund W. 1984. New genera and species of coelacanths from the Bear Gulch limestone (Lower Carboniferous) of Montana (U.S.A.). Geobios 17 (2): 237-244.

https://doi.org/10.1016/S0016-6995(84)80145-X

Lund R. \& Zangerl R. 1974. Squatinactis caudispinatus, a new elasmobranch from the Upper Mississippian of Montana. Annals of Carnegie Museum 45: 43-54.

Lund R., Grogan E.D. \& Fath M. 2014. On the relationships of the Petalodontiformes (Chondrichthyes). Paleontological Journal 48 (9): 1015-1029. https://doi.org/10.1134/S0031030114090081

Lütken C. 1871. On the limits and classification of the Ganoids. Annals and Magazine of Natural History (series 4) 7 (41): 329-339. [New names probably dating from Om Ganoidernes Begraendsning og Indeling, Copenhagen, 1869.] https://doi.org/10.1080/00222937108696386

Lydekker R. 1889. Fishes. In: Nicholson H.A. \& Lydekker R. A Manual of Palceontology for the Use of Students, with a General Introduction on the Principles of Palaeontology, Volume II. $3^{\text {rd }}$ edition: 911-1017. Blackwood and Sons, Edinburgh / London.

Available from https://www.biodiversitylibrary.org/item/125073 [accessed 17 Jul. 2018].

Ma F.-C. 1983. Early Cretaceous primitive teleosts from the Jiaohe Basin of Jilin Province, China. Vertebrata PalAsiatica 21 (1): 17-31. [In Chinese, with English summary.] Available from http://www.ivpp.cas.cn/cbw/gjzdwxb/xbwzxz/200909/P020090917543384453627.pdf [accessed 17 Jul. 2018].

Ma F.-C. 1993. Late Mesozoic Fossil Fishes from the Jiuquan Basin of Gansu Province, China. China Ocean Press, Beijing. [In Chinese, with English summary.]

Maisey J.G. 1982. Studies on the Paleozoic selachian genus Ctenacanthus Agassiz: No. 2 Bythiacanthus St. John and Worthen, Amelacanthus new genus, Eunemacanthus St. John and Worthen, Sphenacanthus Agassiz, and Wodnika Muster. American Museum Novitates (2722): 1-24.

Available from http://hdl.handle.net/2246/3536 [accessed 17 Jul. 2018].

Maisey J.G. (ed.) 1991. Santana Fossils: an Illustrated Atlas. T.F.H. Publications, Neptune City, USA. 
Maisey J.G. 2010. Heslerodidae (Chondrichthyes, Elasmobranchii), a new family of Paleozoic phalacanthous sharks. Kirtlandia (57): 13-21.

Available from https://www.biodiversitylibrary.org/page/51811302 [accessed 17 Jul. 2018].

Maisey J.G., Naylor G.J.P. \& Ward D.J. 2004. Mesozoic elasmobranchs, neoselachian phylogeny and the rise of modern elasmobranch diversity. In: Arratia G. \& Tintori A. (eds) Mesozoic Fishes 3, Systematics, Paleoenvironments and Biodiversity: 17-56. Verlag Dr. Friedrich Pfeil, Munich.

Marck W. von der 1863. Fossile Fische, Krebse und Pflanzen aus dem Plattenkalk der jüngsten Kreide in Westphalen (Schluss). Palaeontographica 11 (2): 41-83. [July; author as W. von der Mark.] Available from https://biodiversitylibrary.org/page/12203336 [accessed 16 Sep. 2018].

Märss T. 1999. A new Late Silurian or Early Devonian thelodont from the Boothia Peninsula, Arctic Canada. Palaeontology 42 (6): 1079-1099. http://doi.org/10.1111/1475-4983.00111

Märss T. 2001. Andreolepis (Actinopterygii) in the Upper Silurian of northern Eurasia. Proceedings of the Estonian Academy of Sciences, Geology 50 (3): 174-189.

Märss T. 2005. Thelodont Oeselia mosaica gen. et sp. nov. from the Wenlock and Ludlow of the East Baltic. Proceedings of the Estonian Academy of Sciences, Geology 54 (3): 181-190.

Märss T. 2006. Thelodonts (Agnatha) from the basal beds of the Kuressaare Stage, Ludlow, Upper Silurian of Estonia. Proceedings of the Estonian Academy of Sciences, Geology 55: 43-66.

Märss T. \& Gagnier P.-Y. 2001. A new chondrichthyan from the Wenlock, Lower Silurian, of Baillie-Hamilton Island, the Canadian Arctic. Journal of Vertebrate Paleontology 21 (4): 693-701. https://doi.org/10.1671/0272-4634(2001)021[0693:ANCFTW]2.0.CO;2

Märss T. \& Karatajūtè-Talimaa V.N. 2002. Ordovician and Lower Silurian thelodonts from Severnaya Zemlya Archipelago (Russia). Geodiversitas 24 (2): 381-404.

Available from http://sciencepress.mnhn.fr/sites/default/files/articles/pdf/g2002n2a6.pdf [accessed 17 Jul. 2018].

Märss T. \& Karatajūtè-Talimaa V.N. 2009. Late Silurian-Early Devonian tessellated heterostracan Oniscolepis Pander, 1856 from the East Baltic and North Timan. Estonian Journal of Earth Sciences 58 (1): 43-62.

Available from http://www.kirj.ee/public/Estonian_Journal_of_Earth_Sciences/2009/issue_1/earth2009-1-43-62.pdf [accessed 17 Jul. 2018].

Märss T., Wilson M.V.H. \& Thorsteinsson R. 2002. New thelodont (Agnatha) and possible chondrichthyan (Gnathostomata) taxa established in the Silurian and Lower Devonian of the Canadian Arctic Archipelago. Proceedings of the Estonian Academy of Sciences, Geology 51: 88-120.

Märss T., Turner S. \& Karatajūtè-Talimaa V.N. 2007. "Agnatha” II Thelodonti. In: Schultze H.-P. (ed.) Handbook of Paleoichthyology Volume 1B: 1-143. Verlag Dr. Friedrich Pfeil, Munich.

Martill D.M., Del Strother P.J.A. \& Gallien F. 2014. Acanthorhachis, a new genus of shark from the Carboniferous (Westphalian) of Yorkshire, England. Geological Magazine 151 (3): 517-533. https://doi.org/10.1017/S0016756813000447

Martin M. 1982a. Nouvelles données sur la phylogénie et la systématique des Dipneustes postpaléozoïques. Comptes rendus de l'Académie des Sciences (série 3) 294: 413-416.

Available from https://gallica.bnf.fr/ark:/12148/bpt6k56537784/f617.image.r=Nouvelles\%20 donn $\%$ C3\%A9es\%20sur\%201a\%20phylog\%C3\%A9nie\%20et $\% 201$ a $\% 20$ syst $\%$ C3\%A9matique $\% 20$ des\%20Dipneustes\%20postpal\%C3\%A9ozo\%C3\%AFques?rk=21459;2 [accessed 16 Sep. 2018]. 
Martin M. 1982b. Nouvelles données sur la phylogénie et la systématique des Dipneustes postpaléozoiques, conséquences stratigraphiques et paléogéographiques. Geobios 15 (Supplement 1): 53-64. https://doi.org/10.1016/S0016-6995(82)80102-2

M'Coy F. 1848. On some new fossil fish of the Carboniferous Period. Annals and Magazine of Natural History (series 2) 2 (7): 1-10. https://doi.org/10.1080/03745485809496133

Mendiola C. 1995. Familia Zygzabatidae n. (Batomorphii, Myliobatoidea): Zygzabatis maroccana n. gen., n. sp. Revista de la Societat Paleontológica d'Elx, Sección Paleontológica (1995): 1-4.

Miles R.S. 1962. Gemuendenaspis n. gen., an arthrodiran fish from the Lower Devonian Hunsrückschiefer of Germany. Transactions of the Royal Society of Edinburgh 65 (3): 59-77.

https://doi.org/10.1017/S0080456800012357

Miles R.S. 1969. Features of placoderm diversification and the evolution of the Arthrodire feeding mechanism. Transactions of the Royal Society of Edinburgh 68 (6): 123-170.

https://doi.org/10.1017/S0080456800014629

Miles R.S. 1971. Acanthodii. In: McGraw-Hill Encyclopedia of Science and Technology, Volume I. $3^{\text {rd }}$ edition: 28-29. McGraw-Hill, New York.

Miles R.S. 1977. Dipnoan (lungfish) skulls and the relationships of the group: a study based on new species from the Devonian of Australia. Zoological Journal of the Linnean Society 61 (1-3): 1-328. https://doi.org/10.1111/j.1096-3642.1977.tb01031.x

Minikh A.V. 1986. Novyj rod paleoniskov iz posdnej permi Yuzhnogo Priural'ya [A new genus of palaeoniscids from Late Permian of Southern Cis-Urals]. Izdatel'stvo Saratovskogo Universiteta [Saratov State University press], Saratov. [In Russian, author also seen as Minich.]

Minikh A.V. 1990. Novyj paleonisk iz pozdnej Permi Vostochno-Evropeiskoj platformy [A new palaeoniscid from the East European Late Permian]. Paleontologicheskii Zhurnal 1990 (3): 71-76. [In Russian.]

Minikh A.V. 1998. Novy'e predstaviteli luchepiorych ryb (otryad Discordichthyida, ord. nov.) iz verkhnej permi Vostochno-Evropeiskoj platformy [New representatives of actinopterygian fish (order Discordichthyida, ord. nov.) from the Upper Permian of the East European platform]. Voprosy Paleontologii i Stratigrafii, Novaja Serija [Problems in Palaeontology and Stratigraphy, new series] 1: 47-58. [In Russian; not seen.]

Minikh M.G. \& Minikh A.V. 1990. Reviziya nekotorykh paleoniskov i novyye ryby iz verkhney permi Vostochno-Yevropeyskoy platformy i vozmozhnosti ispol'zovaniya ikh v stratigrafii [Revision of some palaeoniscids and new fishes from the upper Permian of the East European platform and the possibility of using them in stratigraphy]. In: Aleshechkin O.I. (ed.) Voprosy geologii Yuzhnogo Urala i Nizhnego Povolzh'ya [Problems in the Geology of the Southern Urals and the Lower Volga region]: 84-104. Izdatel'stvo Saratovskogo Universiteta [Saratov State University press], Saratov. [In Russian; not seen.]

Moloshnikov S.V. 2011. Bothriolepiform antiarchs (Pisces, Placodermi) from the Devonian of Central Kazakhstan. Paleontological Journal 45 (3): 291-304. https://doi.org/10.1134/S0031030111030099

Moloshnikov S.V. 2012. Middle-late devonian placoderms (Pisces: Antiarchi) from Central and Northern Asia. Paleontological Journal 46 (10): 1097-1196. https://doi.org/10.1134/S0031030112100012

Monsch K.A. \& Bannikov A.F. 2012. New taxonomic synopses and revision of the scombroid fishes (Scombroidei, Perciformes), including billfishes, from the Cenozoic of territories of the former USSR. Earth and Environmental Science Transactions of The Royal Society of Edinburgh 102 (4): 253-300. https://doi.org/10.1017/S1755691011010085 
Morris J. 1843. A Catalogue of British Fossils, Comprising the Genera and Species hitherto Described with References to their Geological Distribution and to the Localities in which they have been found. Van Voorst, London. https://doi.org/10.5962/bhl.title.112423

Morris J. 1856. A Catalogue of British Fossils, Comprising the Genera and Species hitherto Described with References to their Geological Distribution and to the Localities in which they have been found. Second edition. Van Voorst, London.

Moy-Thomas J.A. 1936. The structure and affinities of the fossil elasmobranch fishes from the Lower Carboniferous Rocks of Glencartholm, Eskdale. Proceedings of the Zoological Society of London 106 (3): 761-788. https://doi.org/10.1111/j.1469-7998.1936.tb06287.x

Moy-Thomas J.A. 1937. The Palaeoniscids from the cement stones of Tarras Waterfoot, Eskdale, Dumfriesshire. Annals and Magazine of Natural History (series 10) 20 (117): 345-356. https://doi.org/10.1080/00222933708655349

Moy-Thomas J.A. 1939. Palaeozoic Fishes. Methuen \& Co, London.

Moy-Thomas J.A. \& Miles R.S. 1971. Palaeozoic Fishes. Second edition. Chapmann \& Hall, London.

Müller J. 1846. Über den Bau und die Grenzen der Ganoiden und über das natürliche System der Fische. Abhandlungen der Königlichen Akademie der Wissenschaften zu Berlin [Aus dem Jahre 1844]: 117216. Available from https://biodiversitylibrary.org/page/30226042 [accessed 28 Aug. 2018].

Murray A.M. \& Cumbaa S.L. 2013. Early Turonian acanthomorphs from Lac des Bois, Northwest Territories, Canada. Journal of Vertebrate Paleontology 33 (2): 293-300.

https://doi.org/10.1080/02724634.2013.722574

Murray A.M. \& Wilson M.V.H. 2013. Two new paraclupeid fishes (Clupeomorpha: Ellimmichthyiformes) from the Upper Cretaceous of Morocco. In: Arratia G., Schultze H.-P. \& Wilson M.V.H. (eds) Mesozoic Fishes 5, Global Diversity and Evolution: 267-290. Verlag Dr. Friedrich Pfeil, Munich.

Mutter R.J., De Blanger K. \& Neuman A.G. 2007. Elasmobranchs from the Lower Triassic Sulphur Mountain Formation near Wapiti Lake (BC, Canada). Zoological Journal of the Linnean Society 149 (3): 309-337. https://doi.org/10.1111/j.1096-3642.2007.00244.x

Mutter R.J., Neuman A.G. \& De Blanger K. 2008. Homalodontus nom. nov., a replacement name for Wapitiodus Mutter, de Blanger and Neuman, 2007 (Homalodontidae nom. nov.,? Hybodontoidea), preoccupied by Wapitiodus Orchard, 2005. Zoological Journal of the Linnean Society 154 (2): 419-420. https://doi.org/10.1111/j.1096-3642.2008.00488.x

Nazarkin M.V. 2002. Trispinax ladae gen. et sp. nov.: a species of the new family of trachinoid fishes Trispinacidae (Perciformes, Trachinoidei) from the Miocene of Sakhalin Island. Journal of Ichthyology 42 (6): 419-426. [Also in Russian: Voprosy Ikhtiologii 42 (4): 459-467.]

Nelson J.S., Grande T.C. \& Wilson M.V.H. 2016. Fishes of the World. $5^{\text {th }}$ edition. Wiley, Hoboken.

Newberry J.S. 1885. Description of some gigantic placoderm fishes recently discovered in the Devonian of Ohio. Transactions of the New York Academy of Sciences 5: 25-28.

Available from https://biodiversitylibrary.org/page/12628056 [accessed 28 Aug. 2018].

Newberry J.S. 1890. The Paleozoic fishes of North America. Monograph of the United States Geological Survey 16 [for 1889]: 1-340. https://doi.org/10.5962/bhl.title.39658

Newberry J.S. \& Worthen A.H. 1866. Palaeontology of Illinois, Section I. Descriptions of new species of vertebrates, mainly from the sub-Carboniferous limestone and coal measures of Illinois. Geological Survey of Illinois 1866 (2): 9-134.

Available from https://biodiversitylibrary.org/page/40446198 [accessed 28 Aug. 2018]. 
Newman M.J. 2002. A new naked jawless vertebrate from the Middle Devonian of Scotland. Palaeontology 45 (5): 933-941. http://doi.org/10.1111/1475-4983.00269

Nicholson H.A. 1879. A Manual of Palceontology for the Use of Students, with a General Introduction on the Principles of Palaeontology, Volume II: 109-172. $2^{\text {nd }}$ edition. Blackwood and Sons, Edinburgh/London. Available from https://www.biodiversitylibrary.org/item/51564 [accessed 17 Jul. 2018].

Novitskaya L.I. 1968. Novye Amfiaspidy (Heterostraci) iz nizhnego devona Sibiri i klassifikatsiya Amphiaspidiformes [New amphiaspids (Heterostraci) from the Lower Devonian of Siberia, and the classification of the Amphiaspidiformes]. In: Obruchev D.V. (ed.) Ocherki po filogenii i sistematike iskopemykh ryb i beschelyustnykh [Outlines on the Phylogeny and Systematics of the Fossil Fishes and Agnathans]: 43-62. Nauka, Moscow. [In Russian.]

Novitskaya L.I. 1971. Les amphiaspides (Heterostraci) du Dévonien de la Sibérie. Cahiers de paléontologie, Éditions du Centre National de la Recherche Scientifique, Paris. [Author also seen as Novitskaia.]

Novitskaya L.I. 1983. Morfologiya drevnikh beschelyustnykh (geterostraki i problema svyazi beschelyustnykh i chelyustnorotykh pozvonochnykh) [Morphology of Fossil Agnathans (Heterostracans and the Problem of Relationship of Jawless and Jawed Vertebrates) / Morphology of Ancient Agnathans (Heterostracans and the Relationship Problem of Agnathans and Gnathostome Vertebrates)]. Transactions of the Palaeontological Institute (Academy of Sciences of the USSR) / Trudy Paleontologicheskogo Instituta 196, Akademia Nauk SSSR. [In Russian.]

Novitskaya L.I. 1986. [The Earliest Agnathans of the USSR. Heterostracans: Cyathaspids, Amphiaspids, Pteraspids]. Transactions of the Palaeontological Institute (Academy of Sciences of the USSR) / Trudy Paleontologicheskogo Instituta 219, Akademia Nauk SSSR. [In Russian.]

Novitskaya L.I. 2004. [Agnathans and early fishes: subclass Heterostraci (heterostracans)]. In: Novitskaya L.I. \& Afanassieva O.B. (eds) Iskopayemyye pozvonochnyye Rossii i sopredel'nykh stran. Beschelyustnyye i drevniye ryby. Spravochnik dlya paleontologov, biologov i geologov [Fossil Vertebrates of Russia and Adjacent Countries. The Reference Book for Paleontologists, Biologists and Geologists]: 69-207. GEOS, Moscow. [In Russian.]

Nursall J.R. 1996. The phylogeny of pycnodont fishes. In: Arratia G. \& Viohl G. (eds) Mesozoic Fishes Systematics and Paleoecology, Proceedings of the International Meeting Eichstätt 1993: 125-152. Verlag Dr. Friedrich Pfeil, Munich.

Nursall J.R. 1999. The pycnodontiform bauplan: the morphology of a successful taxon. In: Arratia G. \& Schultze H.-P. (eds) Mesozoic Fishes 2, Systematics and Fossil Record, Proceedings of the International Meeting Buckow 1997: 189-214. Verlag Dr. F. Pfeil, Munich.

Nursall J.R. \& Capasso L. 2004. Gebrayelichthys (novum), an extraordinary genus of neopterygian fishes from the Cenomanian of Lebanon. In: Arratia G. \& Tintori A. (eds) Mesozoic Fishes 3, Systematics, Paleoenvironments and Biodiversity: 317-340. Verlag Dr. Friedrich Pfeil, Munich.

Obrhelová N. 1961. Vergleichende Osteologie der tertiären Süßwasserfische Böhmens (Gobioidei). Sbornik Ústředního Ústavu Geologického, Oddíl paleontologický 26 (2): 103-192.

Obruchev D.V. 1933. Holonemidae des russischen Devons. Travaux de l'Institut paléozoologique Académie des Sciences de l'URSS 2: 97-116. [Author also seen as Obrutschew or Obručev.]

Obruchev D.V. 1939. Devonskiiye ryby s reki Kureiki [The Devonian Fishes from the Kureyka River]. In: Sbornik Akademiku V.A. Obruchevu [Obruchev Jubilee] 2: 315-330. [In Russian.] 
Obruchev D.V. 1949. [Type Vertebrata]. In: Luppov N.P. (ed.) Atlas rukovodyashchikh form iskopaemykh faun SSSR, Tom 2 Silurijskaya sistema [Atlas of Index Fossils of the Fossil Faunas of the USSR, Volume 2 Silurian System]: 316-328. Izdatel'stvo 'Nauka', Moscow. [In Russian.]

Obruchev D.V. 1964a. [Branch Agnatha]. In: Obruchev D.V. (ed.) Osnovy paleontologii. Vol. XI Agnatha, Pisces: 34-116. Izdatel'stvo 'Nauka', Moscow. [In Russian, translated 1967, Fundamentals of Paleontology, Vol. XI. Agnatha, Pisces. Israel Program for Scientific Translations, Jerusalem.]

Obruchev D.V. 1964b. [Class Placodermi]. In: Obruchev D.V. (ed.) Osnovy paleontologii. Vol. XI Agnatha, Pisces: 118-172. Izdatel'stvo 'Nauka', Moscow. [In Russian, translated 1967, Fundamentals of Paleontology, Vol. XI. Agnatha, Pisces. Israel Program for Scientific Translations, Jerusalem.]

Obruchev D.V. 1964c. [Holocephali]. In: Obruchev D.V. (ed.) Osnovy paleontologii. Vol. XI Agnatha, Pisces: 238-266. Izdatel'stvo 'Nauka', Moscow. [In Russian, translated 1967, Fundamentals of Paleontology, Vol. XI. Agnatha, Pisces. Israel Program for Scientific Translations, Jerusalem.]

Olson E.C. 1951. Vertebrates from the Choza Formation, Permian of Texas. The Journal of Geology 59 (2): 178-181. Available from http://www.jstor.org/stable/30070479 [accessed 17 Jul. 2018].

Owen R. 1840-1845. Odontography or a Treatise on the Comparative Anatomy of the Teeth. Bailliere, London. [Dates as reported by Woodward \& Sherborn 1890: xxix.] https://doi.org/10.5962/bhl.title.113905

Owen R. 1846. Lectures on the Comparative Anatomy and Physiology of the Vertebrate Animals delivered at the Royal College of Surgeons of England 1844 and 1846. Part I. Fishes. Longman, Brown, Green and Longmans, London. https://doi.org/10.5962/bhl.title.113850

Owen R. 1860. Palaeontology, or a Systematic Summary of Extinct Animals and their Geological Relations. A. and C. Black, Edinburgh. [before 14 April] https://doi.org/10.5962/bhl.title.13917

Owen R. 1861. Palaeontology, or a Systematic Summary of Extinct Animals and their Geological Relations, Second edition. A. and C. Black, Edinburgh. https://doi.org/10.5962/bhl.title.61804

Owen R. 1867. On the mandible and mandibular teeth of Cochliodonts. Geological Magazine (Decade 1) 4 (32): 59-63. Available from https://biodiversitylibrary.org/page/32511894 [accessed 27 Jul. 2018].

Pan J. 1992. New Galeaspids (Agnatha) from the Silurian and Devonian of China. Geological Publishing House, Beijing. [Author also seen as P'an K.]

Pan J. \& Chen L. 1993. Geraspididae, a new family of Polybranchiaspidida (Agnatha) from Silurian of northern Anhui. Vertebrata PalAsiatica 31 (3): 225-230. [In Chinese, with English summary.] Available from http://www.ivpp.cas.cn/cbw/gjzdwxb/xbwzxz/200812/W020090813370697520359.pdf [accessed 17 Jul. 2018].

Pan J. \& Wang S.T. 1978. [Devonian Agnatha and Pisces of South China]. In: Symposium on the Devonian System of South China 1974, Institute of Geology and Mineral Resources, Chinese Academy of Geological Sciences: 298-333. Geological Publishing House, Beijing. [In Chinese.]

Pan J. \& Wang S.T. 1980. New finding of Galeaspidiformes in South China. Acta Palaeontologica Sinica 19 (1): 1-7. [In Chinese, with English summary.]

Pan J. \& Wang S.T. 1981. New discoveries of polybranchiaspids from Yunnan Province. Vertebrata PalAsiatica 19 (2): 113-121. [In Chinese, with English summary.]

Available from http://www.ivpp.cas.cn/cbw/gjzdwxb/xbwzxz/200904/W020090813375229410576.pdf [accessed 17 Jul. 2018].

Pan J. \& Wang S.T. 1982. A nomenclatural note on Duyunolepis for Duyunaspis P'an et Wang, 1978. Vertebrata PalAsiatica 20 (4): 370. 
Pan J. \& Wang S.T. 1983. Xiushuiaspidae, a new family of Polybranchiaspiformes from Xiushui of Jiangxi province. Acta Palaeontologica Sinica 22 (5): 505-510. [In Chinese, with English summary.]

Pan J. \& Zeng X.-Y. 1985. Dayongaspidae, a new family of Polybranchiaspiformes (Agnatha) from Early Silurian of Hunan, China. Vertebrata PalAsiatica 23 (3): 207-213. [In Chinese, with English summary.]

Available from http://www.ivpp.cas.cn/cbw/gjzdwxb/xbwzxz/200903/W020090813373989382150.pdf [accessed 17 Jul. 2018].

Pan J., Wang S.T. \& Liu Y.P. 1975. [Early Devonian Agnatha and Pisces of South China / The Lower Devonian Agnatha and Pisces from South China]. Professional Papers on Stratigraphy and Palaeontology 1: 135-169. [In Chinese.]

Pander C.H. 1856. Monographie der fossilen Fische des silurischen Systems der Russisch-Baltischen Gouvernements. Buchdruckerei der Kaiserlichen Akademie der Wissenschaften, St. Petersburg.

Pander C.H. 1857. Über die Placodermen des devonischen Systems. Buchdruckerei der Kaiserlichen Akademie der Wissenschaften, St. Petersburg.

Pander C.H. 1858. Über die Ctenodipterinen des devonischen Systems. Buchdruckerei der Kaiserlichen Akademie der Wissenschaften, St. Petersburg. https://doi.org/10.5962/bhl.title.61017

Pander C.H. 1860. Über die Saurodipterinen, Dendrodonten, Glyptolepiden und Cheirolepiden des devonischen Systems. Buchdruckerei der Kaiserlichen Akademie der Wissenschaften, St. Petersburg.

Patten W. 1931. New Ostracoderms from Oesel. Science 73 (1903): 671-673.

Available from http://www.jstor.org/stable/1655241 [accessed 17 Jul. 2018].

Patterson C. 1964. A review of Mesozoic acanthopterygian fishes with special reference to those of the English Chalk. Philosophical Transactions of the Royal Society, London (Biological sciences) 247 (739): 213-482. Available from http://www.jstor.org/stable/2416611 [accessed 17 Jul. 2018].

Patterson C. 1965. The phylogeny of the Chimaeroids. Philosophical Transactions of the Royal Society, London (Biological Sciences) 249 (757): 101-219. Available from http://www.jstor.org/stable/2416558 [accessed 17 Jul. 2018].

Patterson C. 1967. New Cretaceous berycoid fishes from the Lebanon. Bulletin of the British Museum (Natural History), Geology 14 (3): 67-109.

Available from https://www.biodiversitylibrary.org/page/36368748 [accessed 17 Jul. 2018].

Patterson C. 1970. A clupeomorph fish from the Gault (Lower Cretaceous). Journal of the Linnean Society (Zoology) 49 (3): 161-182. https://doi.org/10.1111/j.1096-3642.1970.tb00733.x

Patterson C. 1993. Osteichthyes. Teleostei. In: Benton M.J. (ed.) The Fossil Record 2: 621-656. Chapman and Hall, London.

Patterson C. \& Rosen D.E. 1977. Review of ichthyodectiform and other mesozoic teleost fishes and the theory and practice of classifying fossils. Bulletin of the American Museum 158 (2): 81-172. Available from http://hdl.handle.net/2246/1224 [accessed 17 Jul. 2018].

Pernègre V.N. \& Elliott D.K. 2008. Phylogeny of the Pteraspidiformes (Heterostraci), Silurian-Devonian jawless vertebrates.Zoologica Scripta37 (4):391-403.https://doi.org/10.1111/j.1463-6409.2008.00333.x

Pfeil F.H. 1983. Zahnmorphologische Untersuchungen an rezenten und fossilen Haien der Ordnungen Chlamydoselachiformes und Echinorhiniformes. Palaeo Ichthyologica 1: 1-315.

Pfeil F.H. 1984. Neoselachian teeth collected from phosphorite-bearing greensand on Chatham Rise east of New Zealand. Geologisches Jahrbuch (Reihe D: Mineralogie, Petrographie, Geochimie, Lagerstättenkunde) 65: 107-115. 
Pictet F.-J. 1850. Description de quelques poissons fossiles du Mont Liban. J.-G. Fick, Genève. https://doi.org/10.5962/bhl.title.8425

Pictet F.-J. 1854. Traité de paléontologie ou Histoire naturelle des animaux fossiles, Tome II. $2^{\text {nd }}$ edition. Baillière, Paris. Available from https://www.biodiversitylibrary.org/item/53455 [accessed 27 Jul. 2018].

Pictet F.-J. \& Humbert A. 1866. Nouvelles recherches sur les poissons fossiles du Mont Liban. Georg, Genève. https://doi.org/10.5962/bhl.title.8429

Poplin C. \& Lund R. 2000. Two new deep-bodied palaeoniscoid actinopterygians from Bear Gulch (Montana, USA, Lower Carboniferous). Journal of Vertebrate Paleontology 20 (3): 428-449. https://doi.org/10.1671/0272-4634(2000)020[0428:TNDBPA]2.0.CO;2

Poyato-Ariza F.J. 1996. A revision of the ostariophysan fish family Chanidae, with special reference to the Mesozoic forms. Palaeo Ichthyologica 6: 1-52.

Poyato-Ariza F.J. \& Wenz S. 2002. A new insight into pycnodontiform fishes. Geodiversitas 24 (1): 139-248. Available from http://sciencepress.mnhn.fr/sites/default/files/articles/pdf/g2002n1a6-low.pdf [accessed 17 Jul. 2018].

Prokofiev A.M. 2002. Morphology and relationships of Neocassandra mica Daniltshenko, 1968 (Pisces; Aulopiformes; Neocassandridae fam. nov.) from the Late Paleocene of Turkmenistan. Paleontological Journal 36 (1): 64-71 [Also in Russian: Paleontologicheskii Zhurnal 2002 (1): 69-76].

Prokofiev A.M. 2004. [Study of the complex of anterior abdominal vertebrae associated with the swimbladder (associated complex) of the Ophidiiformes.] Journal of Natural and Technical Sciences 2004 (2): 129-142. [In Russian, with English summary.]

Prokofiev A.M. 2005. Holosteinae, a new subfamily of paralepidids (Alepisauroidei: Paralepididae). Voprosy ikhtiologii 45 (3): 293-301. [Also in English, Journal of Ichthyology 45 (4): 275-283.]

Prokofiev A.M. 2006. Fossil myctophoid fishes (Myctophiformes: Myctophoidei) from Russia and adjacent regions. Journal of Ichthyology 46 (Suppl. 1): S38-S83.

https://doi.org/10.1134/S0032945206100043

Quenstedt F.A. 1852. Handbuch der Petrefaktenkunde. Laupp'sche Buchhandlung, Tübingen. https://doi.org/10.5962/bhl.title.15107

Quenstedt F.A. 1885. Handbuch der Petrefaktenkunde. $3^{\text {rd }}$ edition. Laupp'sche Buchhandlung, Tübingen. https://doi.org/10.5962/bhl.title.30642

Radovčić J. 1975. Some new Upper Cretaceous teleosts from Yugoslavia with special reference to localities, geology and palaeoenvironment. Palaeontologia Jugoslavia 17: 1-55.

Raposo M.A., Stopiglia R., Brito G.R.R., Bockmann F.A., Kirwan G.M., Gayon J. \& Dubois A. 2017. What really hampers taxonomy and conservation? A riposte to Garnett and Christidis (2017). Zootaxa 4317 (1): 179-184. https://doi.org/10.11646/zootaxa.4317.1.10

Rayner D.H. 1948. The structure of certain Jurassic holostean fishes with special reference to their neurocrania. Philosophical Transactions of the Royal Society of London, Series B, Biological Sciences 233 (601): 287-345. Available from http://www.jstor.org/stable/92370 [accessed 17 Jul. 2018].

Rees J. 2000. A new Pliensbachian (Early Jurassic) neoselachian shark fauna from southern Sweden. Acta Palaeontologica Polonica 45 (4): 407-424.

Available from https://www.app.pan.pl/archive/published/app45/app45-407.pdf [accessed 17 Jul. 2018].

Regan C.T. 1906. A classification of selachian fishes. Proceedings of the Zoological Society of London 1906: 722-758. Available from https://biodiversitylibrary.org/page/31208467 [accessed 28 Aug. 2018]. 
Regan C.T. 1909. On the anatomy and classification of the scombroid fishes. Annals and Magazine of Natural History (series 8) 3 (13): 66-74. https://doi.org/10.1080/00222930908692547

Regan C.T. 1911. The anatomy and classification of the teleostean fishes of the orders Berycomorphi and Xenoberyces. Annals and Magazine of Natural History (series 8) 7 (37): 1-9. https://doi.org/10.1080/00222931108692901

Reif W.E. 1978. Tooth enameloid as a taxonomic criterion. 2. Is "Dalatias" barnstonensis Sykes 1971 (Triassic, England) a squalomorphic shark? Neues Jahrbuch für Geologie und Paläontologie, Monatshefte 1978 (1): 42-58.

Reif W.E. 1980. Tooth enameloid as a taxonomic criterion: 3. A new primitive shark family from the lower Keuper. Neues Jahrbuch für Geologie und Paläontologie, Abhandlungen 160 (2): 61-72.

Ritchie A. 1973. Wuttagoonaspis gen. nov., an unusual arthrodire from the Devonian of Western New South Wales, Australia. Palaeontographica (Abteilung A: Paläozoologie-Stratigraphie) 143 (1-6): 5872 .

Ritchie A. \& Gilbert-Tomlinson J. 1977. First Ordovician vertebrates from the Southern Hemisphere. Alcheringa 1 (4): 351-368. https://doi.org/10.1080/03115517708527770

Robertson G.M. 1935. The ostracoderm order Osteostraci. Science 82: 282-283. Available from http://www.jstor.org/stable/1661217 [accessed 17 Jul. 2018].

Rohon J.V. 1892. Die obersilurischen Fische von Oesel. I. Theil, Thyestidae und Tremataspidae. Mémoires de l'Académie Impériale des Sciences de St. Pétersbourg (7ième série) 38 (13): 1-88. Available from https://biodiversitylibrary.org/page/53791596 [accessed 28 Aug. 2018].

Rohon J.V. 1897. Beiträge zur Classification der palaeozoischen Fische. Věstník Královské České Společnosti Náuk, Tř́lda Mathematicko-Přrrodovedecká [Sitzungsberichte der Königlichen Böhmischen Gesellschaft der Wissenschaften Mathematisch-Naturwissenschaftliche Classe] (XXXVII) [for 1896]: 1-33. Available from https://biodiversitylibrary.org/page/4136706 [accessed 28 Aug. 2018].

Romer A.S. 1945. Vertebrate Paleontology. Second edition. University of Chicago Press, Chicago.

Romer A.S. 1947. Review of the Labyrinthodontia. Bulletin of the Museum of Comparative Zoology at Harvard College 99 (1): 1-368.

Available from https://biodiversitylibrary.org/page/4322028 [accessed 2 Aug. 2018].

Romer A.S. 1966. Vertebrate paleontology. Third edition. University of Chicago Press, Chicago / London.

Ruggiero M.A., Gordon D.P., Orrell T.M., Bailly N., Bourgoin T., Brusca R.C., Cavalier-Smith T., Guiry M.D. \& Kirk P.M. 2015. A higher level classification of all living organisms. PLoS One 10 (4): e0119248. https://doi.org/10.1371/journal.pone.0119248

Saint-Seine P. de 1949. Les poissons des calcaires lithographiques de Cerin (Ain). Nouvelles archives du Muséum d'Histoire naturelle de Lyon 2: 1-357.

Saint-Seine P. de 1955. Poissons fossiles de l'étage de Stanleyville (Congo belge). I. La faune des argilites et schistes bitumineux. Annales du Musée de Congo Belge, Série in $8^{\circ}$, Sciences géologiques 14: $1-126$.

Sansom I.J., Aldridge R.J. \& Smith M.M. 2000. A microvertebrate fauna from the Llandovery of South China. Transactions of the Royal Society of Edinburgh, Earth Sciences 90 (3): 255-272. https://doi.org/10.1017/S0263593300002595

Sansom R.S. 2009. Phylogeny, classification and character polarity of the Osteostraci (Vertebrata). Journal of Systematic Palaeontology 7 (1): 95-115. https://doi.org/10.1017/S1477201908002551 
Santini F. \& Tyler J.C. 2003. A phylogeny of the families of fossil and extant tetraodontiform fishes (Acanthomorpha, Tetraodontiformes), Upper Cretaceous to Recent. Zoological Journal of the Linnean Society 139 (4): 565-617. https://doi.org/10.1111/j.1096-3642.2003.00088.x

Santini F. \& Tyler J.C. 2004. The importance of even highly incomplete fossil taxa in reconstructing the phylogenetic relationships of the Tetraodontiformes (Acanthomorpha: Pisces). Integrative and Comparative Biology 44 (5): 349-357. https://doi.org/10.1093/icb/44.5.349

Sauvage H.-E. 1875. Note sur le genre Nummopalatus et sur les espèces de ce genre trouvées dans les terrains tertiaires de la France. Bulletin de la Société géologique de France (série 3) (3): 613-630. Available from https://biodiversitylibrary.org/page/54886641 [accessed 29 Aug. 2018].

Schaeffer B. 1949. A teleost from the Livingston Formation of Montana. American Museum Novitates (1427): 1-16. Available from http://hdl.handle.net/2246/2348 [accessed 17 Jul. 2018].

Schaeffer B. 1968. A new actinopterygian fish from the Cretaceous of North America. American Museum Novitates (2344): 1-10. Available from http://hdl.handle.net/2246/2554 [accessed 27 Jul. 2018].

Schaeffer B. \& Patterson C. 1984. Jurassic fishes from the western United States, with comments on Jurassic fish distribution. American Museum Novitates (2796): 1-86. Available from http://hdl.handle.net/2246/5270 [accessed 27 Jul. 2018].

Schultz L.P. with Stern E. 1948. The Ways of Fishes. Van Norstrand, New York (Toronto and London).

Schultze H.-P. 1993. Osteichthyes: Sarcopterygii. In: Benton M.J. (ed.) The Fossil Record 2: 657-663. Chapman and Hall, London.

Schultze H.-P., Stewart J.D., Neuner A.M. \& Coldiron R.W. 1982. Type and figured specimens of fossil vertebrates in the collection of the University of Kansas Museum of Natural History Part I Fossil Fishes. University of Kansas Museum of Natural History Miscellaneous publication (73): 1-53. Available from https://biodiversitylibrary.org/page/5783666 [accessed 17 Jul. 2018].

Schwarzhans W. 1996. Otoliths from the Maastrichtian of Bavaria and their evolutionary significance. In: Arratia G. \& Viohl G. (eds) Mesozoic Fishes Systematics and Paleoecology, Proceedings of the International Meeting Eichstätt 1993: 417-431. Verlag Dr. Friedrich Pfeil, Munich.

Scott B.R. \& Wilson M.V.H. 2014. The Superciliaspididae, a new family of Early Devonian Osteostraci (jawless vertebrates) from northern Canada, with two new genera and three new species. Journal of Systematic Palaeontology 13 (3) [for 2015]: 167-187. [Published online 12 March 2014.] https://doi.org/10.1080/14772019.2013.863809

Sedgwick A. \& M'Coy F. 1855. Synopsis of the Classification of the British Paleozoic Rocks, with a Systematic Description of the British Palaeozoic Fossils in the Geological Museum of the University of Cambridge. Parker and Son, London. Available from https://biodiversitylibrary.org/page/14970842 [accessed 17 Jul. 2018].

Selezneva A.A. 1985. Evenkia - ancestor of Polypterus (Actinopterygii). Paleontologicheskii Zhurnal 1985 (3): 71-76. [In Russian, English translation in Paleontological Journal 19: 1-6; author formerly known as Kazantseva or Kazantseva-Selezneva.]

Sferco E., López-Arbarello A. \& Báez A.M. 2015. Phylogenetic relationships of †Luisiella feruglioi (Bordas) and the recognition of a new clade of freshwater teleosts from the Jurassic of Gondwana. BMC Evolutionary Biology 15: 268. https://doi.org/10.1186/s12862-015-0551-6

Sheiko B.A. 2013. On family-group names of extant fishes and fish-like vertebrates of the world. Issledovaniya fauny morey [Explorations of the Fauna of the Seas], Zoological Institute RAS, St. Petersburg 74 (82): 1-204. 
Sherborn C.D. 1922. Index Animalium, sectio secunda. British Museum, London. Available from https://biodiversitylibrary.org/page/36192110 [accessed 17 Jul. 2018].

Shimada K., Popov E.V., Siversson M., Welton B.J. \& Long D.J. 2015. A new clade of putative planktonfeeding sharks from the Upper Cretaceous of Russia and the United States. Journal of Vertebrate Paleontology 35 (5): e981335. https://doi.org/10.1080/02724634.2015.981335

Shu D.-G. 2003. A paleontological perspective of vertebrate origin. Chinese Science Bulletin 48 (8): 725-735. https://doi.org/10.1007/BF03187041

Shu D.-G., Luo H.-L., Conway Morris S., Zhang X.-L., Hu S.-X., Chen L., Han J., Zhu M., Li Y. \& Chen L.-Z. 1999. Lower Cambrian vertebrates from south China. Nature 402: 42-46. https://doi.org/10.1038/46965

Silva Santos R. da 1985a. Araripichthys castilhoi novo gênero e especie de teleostei da Formação Santana, Chapada do Araripe, Brasil. In: Campos D. de A., Ferreira C.S., Brito I.M. \& Viana C.F. (eds) Coletânea de Trabalhos Paleontológicos Série Geologia 27: 133-139. Ministério das Minas e EnergiaDepartamento Nacional de Produção Mineral, Rio de Janeiro.

Silva Santos R. da 1985b. Laeliichthys ancestralis, novo gênero e espécie de Osteoglossiformes do Aptiano da Formação Areado, Estado de Minas Gerais, Brasil. In: Campos D. de A., Ferreira C.S., Brito I.M. \& Viana C.F. (eds) Coletânea de Trabalhos Paleontológicos Série Geologia 27: 161-167. Ministério das Minas e Energia-Departamento Nacional de Produção Mineral, Rio de Janeiro.

Silva Santos R. da 1990. Vinctifer longirostris Silva Santos, 1972, do Cretáceo inferior da Formação Marizal, Estado da Bahia, Brazil. Anais da Academia Brasileira de Ciências 62 (3): 251-260.

Silva Santos R. da \& Silva Corréa V. L. da 1985. Contribuição ao conhecimento da paleoictiofaúnula do Cretáceo do Brasil. In: Campos D. de A., Ferreira C.S., Brito I.M. \& Viana C.F. (eds) Coletânea de Trabalhos Paleontológicos Série Geologia 27 (2): 169-174. Ministério das Minas e EnergiaDepartamento Nacional de Produção Mineral, Rio de Janeiro.

Silva Santos R. da \& Travassos H. 1960. Contribuição à paleontologia do estado do Pará: peixes fósseis da formação Pirabas. Monografia do Instituto Brasileiro de Geografia e Estatística, Divisão de Geologia et Mineralogia 16: 1-35.

Siverson M. 1999. A new large lamniform shark from the uppermost Gearle Siltstone (Cenomanian, Late Cretaceous) of Western Australia. Transactions of the Royal Society of Edinburgh, Earth Sciences 90 (1): 49-66. https://doi.org/10.1017/S0263593300002509

Smith M.M. 1979. Structure and histogenesis of tooth plates in Sagenodus inaequalis Owen considered in relation to the phylogeny of post-Devonian dipnoans. Proceedings of the Royal Society of London, Series B, Biological Sciences 204 (1154): 15-39. Available from http://www.jstor.org/stable/77485 [accessed 17 Jul. 2018].

Snitting D. \& Blom H. 2009. Correcting taxon names containing diacritics - examples from Paleozoic vertebrates. Journal of Vertebrate Paleontology 29 (1): 269-270.

https://doi.org/10.1080/02724634.2009.10010377

Soehn K.L, Märss T., Caldwell M.W. \& Wilson M.V.H. 2001. New and biostratigraphically useful thelodonts from the Silurian of the Mackenzie Mountains, Northwest Territories, Canada. Journal of Vertebrate Paleontology 21 (4): 651-659.

https://doi.org/10.1671/0272-4634(2001)021[0651:NABUTF]2.0.CO;2

Soler-Gijón R. 1997. New discoveries of xenacanth sharks from the Late Carboniferous of Spain (Puertollano Basin) and Early Permian of Germany (Saar-Nahe Basin): implications for the phylogeny 
of xenacanthiform and anacanthous sharks. Neues Jahrbuch für Geologie and Paläontologie, Abhandlungen 205 (1): 1-31.

Sorbini L. \& Bannikov A.F. 1991. The Cretaceous fishes of Nardò $2^{\circ}$ : An enigmatic spiny-rayed fish. Bollettino della Società Paleontologica Italiana 30 (2): 239-249.

Sorbini L. \& Bannikov A.F. 1996. A new percopsiform-like paracanthopterygian fish from the Early Paleocene of Trieste province, north-eastern Italy. Atti del Museo Civico di Storia Naturale di Trieste 47: 309-317.

Sorbini L., Boscaini E. \& Bannikov A.F. 1991. On the morphology and systematics of the Eocene fish genus Tortonesia Sorbini from Bolca. Miscellanea Paleontologica n. 3. Studi e ricerche sui giacimenti terziari di Bolca VI: 115-132.

Springer V.G. \& Smith-Vaniz W.F. 2008. Supraneural and pterygiophore insertion patterns in carangid fishes, with description of a new Eocene carangid tribe, Paratrachinotini, and a survey of anterior analfin pterygiophore insertion patterns in Acanthomorpha. Bulletin of the Biological Society of Washington 16 (1): 1-73. https://doi.org/10.2988/0097-0298(2008)16[1:SAPIPI]2.0.CO;2

Stahl B.J. 1999. Chondrichthyes III: Holocephali. In: Schultze H.-P. (ed.) Handbook of Paleoichthyology Volume 4: 1-164. Verlag Dr. Friedrich Pfeil, Munich.

Stamberg S. 2006. Carboniferous-Permian actinopterygian fishes of the continental basins of the Bohemian Massif, Czech Republic: an overview. Geological Society of London, Special Publications (265): 217-230. https://doi.org/10.1144/GSL.SP.2006.265.01.10

Steinmann G. \& Döderlein L. 1890. Elemente der Paläontologie. Wilhelm Engelmann, Leipzig. https://doi.org/10.5962/bhl.title.15050

Stensiö E. 1916, see Andersson E.

Stensiö E. 1921. Triassic Fishes from Spitzbergen, Part I. Adolf Holzhausen, Vienna.

Stensiö E. 1925. Triassic Fishes from Spitzbergen, Part II. Kungliga Svenska Vetenskapsakademiens Handlingar 3 (2): 1-261.

Stensiö E. 1931. Upper Devonian vertebrates from East Greenland, collected by the Danish Greenland expeditions in 1929 and 1930. Meddelelser om Grønland 86 (1): 1-212.

Stensiö E. 1932a. Triassic fishes from East Greenland, collected by the Danish Greenland expeditions in 1929-1931. Meddelelser om Grønland 83 (3): 1-305.

Stensiö E. 1932b. The Cephalaspids of Great Britain. British Museum (Natural History), London. https://doi.org/10.5962/bhl.title.118830

Stensiö E. 1937. On the Devonian coelacanthids of Germany with special reference to the dermal skeleton. Kungliga Svenska Vetenskapsakademiens Handlingar (series 3) 16 (4): 1-56.

Stensiö E. 1939. A new anaspid from the Upper Devonian of Scaumenac Bay in Canada, with remarks on the other anaspids. Kungliga Svenska Vetenskapsakademiens Handlingar (series 3) 18 (1): 1-25.

Stensiö E. 1944. Contributions to the knowledge of the vertebrate fauna of the Silurian and Devonian of Western Podolia II: Notes on two arthrodires from the Downtonian of Podolia. Arkiv för Zoologi 35A (9): $1-83$.

Stensiö E. 1945. On the head of certain arthrodires. II. On the cranium and cervical joint of the Dolichothoraci (Acanthaspida). Kungliga Svenska Vetenskapsakademiens Handlingar (series 3) 22: $1-70$. 
Stensiö E. 1948. On the Placodermi of the Upper Devonian of East Greenland. II. Antiarchi: subfamily Bothriolepinae, with an attempt at a revision of the previously described species of that family. Meddelelser om Grønland 139: 1-622.

Stensiö E. 1958. Les cyclostomes fossils ou ostracodermes. In: Grasse P.P. (ed.) Traité de Zoologie Tome 13 Fascicule 1: 173-425. Masson and Cie, Paris.

Stensiö E. 1959. On the pectoral fin and shoulder girdle of the arthrodires. Kungliga Svenska Vetenskapsakademiens Handlingar (series 4) 8 (1): 1-229.

Stensiö E. 1963. Anatomical studies on the arthrodiran head. Part I. Preface, geological and geographical distribution, the organisation of the head in the Dolichothoraci, Coccosteomorphi, and Pachyosteomorphi. Taxonomic appendix. Kungliga Svenska Vetenskapsakademiens Handlingar (series 4) 9 (2): 1-419.

Steyskal G.C. 1980. The grammar of family-group names as exemplified by those of fishes. Proceedings of the Biological Society of Washington 93 (1): 168-177.

Available from https://www.biodiversitylibrary.org/page/34557938 [accessed 17 Jul. 2018].

Stiassny M.L.J., Parenti L.R. \& Johnson G.D. (eds) 1996. Interrelationships of Fishes. Academic Press, San Diego.

Stolley E. 1920. Beiträge zur Kenntnis der Ganoiden des deutschen Muschelkalks. Palaeontographica 63 (3/4): 25-86. Available from https://biodiversitylibrary.org/page/35749474 [accessed 16 Sep. 2018].

Strand E. 1932. Miscellanea nomenclatorica zoologica et palaeontologica IV. Folia Zoologica et Hydrobiologica 4 (2): 193-196.

Strand E. 1933. Zoologische und paläontologische Ergebnisse von den Svalbard- und EismeerUntersuchungen Norwegens. Folia Zoologica et Hydrobiologica 5 (1): 118-121.

Strand E. 1934. Zoologische und paläontologische Ergebnisse von den Svalbard- und EismeerUntersuchungen Norwegens, II. Folia Zoologica et Hydrobiologica 5 (2): 326-330.

Strand E. 1942. Nomenklatorische Bemerkungen über einige fossile Fische. Folia Zoologica et Hydrobiologica 11 (2): 383-386.

Su D.-Z. 1980. [On late Mesozoic fish fauna from Sinjiang, China.] Vertebrata PalAsiatica 18 (1): 76-80. [In Chinese.]

Available from http://www.ivpp.cas.cn/cbw/gjzdwxb/xbwzxz/200904/P020100310362926727460.pdf [accessed 17 Jul. 2018].

Su D.-Z. 1985. On late Mesozoic fish fauna from Xinjiang (Sinkiang), China. Memoirs of Institute of Vertebrate Palaeontology and Palaeoanthropology, Academia Sinica (17): 61-136. [In Chinese, with English summary.]

Su T.-T. 1974. New Jurassic ptycholepid fishes from Szechuan, S. W. China. Vertebrata PalAsiatica 12 (1): 1-20. [In Chinese, with English summary.]

Available from http://www.ivpp.cas.cn/cbw/gjzdwxb/xbwzxz/200905/W020090813377660414039.pdf [accessed 17 Jul. 2018].

Sun Z. \& Ni P. 2017. Revision of Kyphosichthys grandei Xu \& Wu, 2012 from the Middle Triassic of Yunnan Province, South China: implications for phylogenetic interrelationships of ginglymodian fishes. Journal of Systematic Palaeontology 16 (1) [for 2018]: 67-85. [Published online 24 Jan. 2017.] https://doi.org/10.1080/14772019.2016.1269049

Sun Z., Lombardo C., Tintori A., Jiang D.-Y., Hao W.-C., Sun Y.-L. \& Lin H.-Q. 2012. Fuyuanperleidus dengi Geng et al., 2012 (Osteichthyes, Actinopterygii) from the Middle Triassic of Yunnan Province, 
South China. Rivista Italiana di Paleontologia e Stratigrafia 118 (3): 359-373. Available from https://riviste.unimi.it/index.php/RIPS/article/view/6011/6029 [accessed 17 Jul. 2018].

Sun Z., Tintori A., Xu Y., Lombardo C., Ni P. \& Jiang D. 2016. A new non-parasemionotiform order of the Halecomorphi (Neopterygii, Actinopterygii) from the Middle Triassic of Tethys, Journal of Systematic Palaeontology 15 (3) [for 2017]: 223-240 [Published online 25 May 2016.] https://doi.org/10.1080/14772019.2016.1181679

Suteethorn S., Le Loeuff J., Buffetaut E., Suteethorn V. \& Wongko K. 2013. First evidence of a mamenchisaurid dinosaur from the Upper Jurassic-Lower Cretaceous Phu Kradung Formation of Thailand. Acta Palaeontologica Polonica 58 (3): 459-469.

Available from https://www.app.pan.pl/archive/published/app58/app20090155.pdf [accessed 17 Jul. 2018].

Sytchevskaya E.K. \& Prokofiev A.M. 2002. First findings of Xiphioidea (Perciformes) in the late Paleocene of Turkmenistan. Voprosy Ikhtiologii 42 (3): 227-237. [In Russian.]

Sytchevskaya E.K. \& Prokofiev A.M. 2003. A new acanthopterygian family, Asianthidae (Perciformes) from the Upper Paleocene of Turkmenistan. Journal of Ichthyology 43 (1): 1-15. [Voprosy Ikhtiologii 43 (1): 5-20.]

Sytchevskaya E.K. \& Prokofiev A.M. 2004. Eels (Anguilliformes) from the Late Palaeocene of Turkmenistan. Journal of Ichthyology 44 (1): 22-35. [Second author as Prokofyev; Voprosy Ikhtiologii 44 (1): 27-41.]

Takai F. 1944. A monograph on the lycopterid fishes from the Mesozoic of eastern Asia. Journal of the Faculty of Science, Imperial University of Tokyo (Sect. 2: Geology, Mineralogy, Geography, Seismology) 6: 207-269.

Tarlo L.B. 1962. The classification and evolution of the Heterostraci (Klasyfikaeja i ewolucja Heterostraci). Acta Palaeontologica Polonica 7 (1-2): 249-290.

Available from https://www.app.pan.pl/archive/published/app07/app07-249.pdf [accessed 17 Jul. 2018].

Tarlo L.B.H. 1965. Psammosteiformes (Agnatha) - a review with description of new material from the Lower Devonian of Poland. I. General part. Palaeontologica Polonica 13: 1-135. Available from http://www.palaeontologia.pan.pl/Archive/1964-13.pdf [accessed 17 Jul. 2018].

Tarlo L.B.H. 1966. Psammosteiformes (Agnatha) - a review with description of new material from the Lower Devonian of Poland. II. Systematic part. Palaeontologica Polonica 15: 1-168. Available from http://www.palaeontologia.pan.pl/Archive/1965-15.pdf [accessed 17 Jul. 2018].

Tavani G. 1955. Osservazioni su alcuni Plectognathi (Gymnodonti). Atti della Società Toscana di Scienze naturali residenti in Pisa Serie A 62: 176-200.

Taverne L. 1973. Sur la position systématique et les affinités de Greenwoodella tockensis Taverne L. \& Ross P.H. 1973. (Pisces Elopiformes) de l'Aptien inférieur de l'Ile d'Helgoland (Allemagne). Bulletin de l'Institut royal des Sciences naturelles de Belgique (Sciences de la Terre) 49 (9): 1-6. Available from http://biblio.naturalsciences.be/rbins-publications/bulletin-of-the-royal-belgian-institute-of-naturalsciences-earth-sciences/49-1973/irscnb_p4087_rbins 17641_49_bulletin-9.pdf [accessed 17 Jul. 2018].

Taverne L. 1974. Sur l'origine des Téléostéens Gonorhynchiformes. Bulletin de la Société belge de géologie, de paléontologie et d'hydrologie 83 (1): 55-60.

Taverne L. 1975. À propos de trois téléostéens Salmoniformes fossiles du Crétacé inférieur (Wealdien) du Zaire, précédemment décrits dans les genres Leptolepis et Clupavus (Pisces, Teleostei). Revue zoologique africaine 89 (3): 481-504. 
Taverne L. 1976. Les téléostéens fossiles du crétacé moyen de Kipala (Kwango, Zaire). Annales du Musée royal de l'Afrique centrale (Série in octavo: Sciences géologiques) 79: 1-50.

Taverne L. 1977. Ostéologie de Clupavus maroccanus (Crétacé supérieur du Maroc) et considérations sur la position systématique et les relations des Clupavidae au sein de l'ordre des Clupeiformes sensu stricto (Pisces, Teleostei). Geobios 10 (5): 697-722. https://doi.org/10.1016/S0016-6995(77)80048-X

Taverne L. 1979. Ostéologie, phylogénèse et systématique des téléostéens fossiles et actuels du superordre des Ostéoglossomorphes. Troisième partie. Évolution des structures ostélogiques et conclusions générales relatives à la phylogénèse et à la systématique du superordre. Addendum. Mémoires de l'Académie royale de Belgique, Classe des Sciences (series 2) 43: 1-168.

Taverne L. 1982. Sur Pattersonella formosa (Traquair R.H., 1911) et Nybelinoides brevis (Traquair R.H., 1911), téléostéens Salmoniformes argentinoides du Wealdien inférieur de Bernissart, Belgique, précédemment attribués au genre Leptolepis Agassiz L., 1832. Bulletin de l'Institut royal des Sciences naturelles de Belgique (Sciences de la Terre) 54 (3): 1-27.

Taverne L. 1997. The Cretaceous fish of Nardo 4: Apulichthys gayeti gen. nov., sp. nov. (Teleostei, Ostariophysi, Gonorhynchiformes). Bollettino del Museo Civico di Storia Naturale di Verona 21: 401436.

Taverne L. 1998. Les ostéoglossomorphes marins de l'Éocène du Monte Bolca (Italie): Monopteros Volta 1796, Thrissopterus Heckel 1856 et Foreyichthys Taverne, 1979. Considérations sur la phylogénie des téléostéens ostéoglossomorphes. Miscellanea Paleontologica n. 4. Studi e ricerche sui giacimenti terziari di Bolca VII: 67-158.

Taverne L. 1999. Les poissons crétacés de Nardò 8: Sorbininardus apuliensis gen. nov., sp. nov. (Teleostei, Ostariophysi, Anotophysi, Sorbininardiformes, nov. ord.). Miscellanea Paleontologica n. 5. Studi e ricerche sui giacimenti terziari di Bolca VIII: 77-103.

Taverne L. 2003. Les poissons crétacés de Nardò $16^{\circ}$ : Sorbinicharax verraesi gen. sp. nov. (Teleostei, Ostariophysi, Otophysi, Characiformes). Bollettino del Museo Civico di Storia Naturale di Verona, Geologia Paleontologia Preistoria 27: 29-45.

Taverne L. 2004a. Les poissons crétacés de Nardò 19: Nardorex zorzini gen. et sp. nov. (Teleostei, Aulopiformes, Alepisauroidei). Bollettino Museo Civico di Storia Naturale di Verona, Geologia Paleontologia Preistoria 28: 29-40.

Taverne L. 2004b. Libanechelys bultyncki gen. et sp. nov., une nouvelle anguille primitive (Teleostei, Anguilliformes) du Cénomanien marin du Liban. Bulletin de l'Institut royal des Sciences naturelles de Belgique (Sciences de la Terre) 74: 73-87.

Available from http://www.vliz.be/imisdocs/publications/ocrd/255046.pdf [accessed 16 Sep. 2018].

Taverne L. 2005. Les poissons crétacés de Nardò $20^{\circ}$ : Chanoides chardoni sp. nov. (Teleostei, Ostariophysi, Otophysi). Bollettino del Museo Civico di Storia Naturale di Verona, Geologia Paleontologia Preistoria 29: 39-54.

Taverne L. 2008. Considerations about the Late Cretaceous genus Chirocentrites and erection of the new genus Heckelichthys (Teleostei, Ichthyodectiformes) - A new visit inside the ichthyodectid phylogeny. Bulletin de l'Institut royal des Sciences naturelles de Belgique (Sciences de la Terre) 78: 209-228. Available from http://www.vliz.be/imisdocs/publications/ocrd/240378.pdf [accessed 16 Sep. 2018].

Taverne L. 2014. Les poissons du Santonien (Crétacé supérieur) d'Apricena (Italie du Sud). $7^{\circ}$ Garganoclupea svetovidovi gen. et sp. nov. et Apricenaclupea ridewoodi gen. et sp. nov. (Teleostei, Clupeiformes). Bollettino del Museo Civico di Storia Naturale di Verona, Geologia Paleontologia Preistoria 38: 27-49. 
Available from http://www.museostorianaturaleverona.it/media/_Musei/_StoriaNaturale/_Allegati/ Biblioteca/Bollettino/Bollettino\%2038/Geologia\%20Preistoria/3._Taverne_Garganoclupea.pdf [accessed 17 Jul. 2018].

Taverne L. \& Capasso L. 2013. Gladiopycnodontidae, a new family of pycnodontiform fishes from the Late Cretaceous of Lebanon, with the description of three genera. European Journal of Taxonomy 57: 1-30. https://doi.org/10.5852/ejt.2013.57

Taverne L. \& Capasso L. 2014. Les poissons crétacés de Nardò 36º Compléments à l'étude de Nardoechelys robinsi Taverne, 2002 (Teleostei, Anguilliformes). Bollettino del Museo Civico di Storia Naturale di Verona, Geologia Paleontologia Preistoria 38: 3-16. Available from http://www.museostorianaturaleverona.it/media/_Musei/_StoriaNaturale/_Allegati/Biblioteca/ Bollettino/Bollettino\%2038/Geologia\%20Preistoria/1._Taverne_Capasso_Nardoechelys.pdf [accessed 17 Jul. 2018]

Taverne L. \& Chanet B. 2000. Faugichthys loryi n. gen., n. sp. (Teleostei, Ichthyodectiformes) de l'Albien terminal (Crétacé inférieur marin) du vallon de la Fauge (Isère, France) et considérations sur la phylogénie des Ichthyodectidae. Geodiversitas 22: 23-34. Available from http://sciencepress.mnhn.fr/sites/default/files/articles/pdf/g2000n1a2.pdf [accessed 17 Jul. 2018].

Taverne L. \& Cosmo P.D. de 2008. Les poissons du Santonien (Crétacé supérieur) d'Apricena (Italie du Sud) $1^{\circ}$ : Chanoides weberi sp. nov. (Teleostei, Ostariophysi, Otophysi, Chanoididae). Bollettino del Museo Civico di Storia Naturale di Verona, Geologia Paleontologia Preistoria 32: 29-38. Available from http://www.museostorianaturaleverona.it/media/_Musei/_StoriaNaturale/_Allegati/Biblioteca/ Bollettino/Bollettino\%2032/Bollettino\%2032\%20blu/05_Taverne_De_Cosmo_29-38.pdf [accessed 17 Jul. 2018].

Taverne L. \& Gayet M. 2005. Phylogenetical relationships and palaeozoogeography of the marine Cretaceous Tselfatiiformes (Teleostei, Clupeocephala). Cybium 29 (1): 65-87. Available from http://sfi-cybium.fr/fr/phylogenetical-relationships-and-palaeozoogeography-marine-cretaceoustselfatiiformes-teleostei [accessed 17 Jul. 2018].

Thiollière V. J. de l'Isle. 1858. Note sur les poissons fossiles du Bugey, et sur l'application de la méthode de Cuvier à leur classement. Bulletin de la Société géologique de France (série 2) 15: 782-793. Available from https://biodiversitylibrary.org/page/54405474 [accessed 29 Aug. 2018].

Thurmond J.T. \& Jones D.E. 1981. Fossil Vertebrates of Alabama. University of Alabama Press, Alabama.

Tinn O. \& Märss T. 2018. The earliest osteostracan Kalanaspis delectabilis gen. et sp. nov. from the mid-Aeronian (mid-Llandovery, lower Silurian) of Estonia, Journal of Vertebrate Paleontology 38 (1): [1-8] e1425212. https://doi.org/10.1080/02724634.2017.1425212

Tintori A. 1981. Two new pycnodonts (Pisces, Actinopterygii) from the Upper Triassic of Lombardy (N. Italy). Rivista Italiana di Paleontologia e Stratigrafia 86 (4) [for 1980]: 795-824.

Tintori A. \& Lombardo C. 1996. Gabanellia agilis gen. n. sp. n. (Actinopterygii, Perleidiformes) from the Calcare di Zorzino of Lombardy (North Italy). Rivista Italiana di Paleontologia e Stratigrafia 102 (2): 227-236. Available from https://riviste.unimi.it/index.php/RIPS/article/view/5249/5273 [accessed 17 Jul. 2018].

Traquair R.H. 1875. On the structure and systematic position of the genus Cheirolepis. Annals and Magazine of Natural History (series 4) 15 (88): 237-249. https://doi.org/10.1080/00222937508681069

Traquair R.H. 1877. On the Agassizian genera Amblypterus, Palaeoniscus, Gyrolepis and Pygopterus. The Quarterly Journal of the Geological Society of London 33: 548-578.

https://doi.org/10.1144/GSL.JGS.1877.033.01-04.33 
Traquair R.H. 1879. On the structure and affinities of the Platysomidae. Transactions of the Royal Society of Edinburgh 29 (1): 343-391. https://doi.org/10.1017/S0080456800028544

Traquair R.H. 1881a. Report on fossil fishes collected by the Geological Survey of Scotland in Eskdale and Liddesdale. Part I. Ganoidei. Transactions of the Royal Society of Edinburgh 30 (1): 15-71. https://doi.org/10.1017/S0080456800028970

Traquair R.H. 1881b. On the cranial osteology of Rhizodopsis. Transactions of the Royal Society of Edinburgh 30 (1): 167-179. https://doi.org/10.1017/S008045680002901X

Traquair R.H. 1888a. On the structure and classification of the Asterolepidae. Annals and Magazine of Natural History (series 6) 2 (12): 485-504. https://doi.org/10.1080/00222938809487519

Traquair R.H. 1888b. Notes on the nomenclature of the fishes of the Old Red Sandstone of Great Britain. Geological Magazine (Decade 3) 5: 507-517.

Available from https://biodiversitylibrary.org/page/30534007 [accessed 17 Jul. 2018].

Traquair R.H. 1889. On the "Dendrodont" fishes. Geological Magazine (Decade 3) 6: 490-492. Available from https://biodiversitylibrary.org/page/30859925 [accessed 27 Jul. 2018].

Traquair R.H. 1890. Notes on the Devonian fishes of Scaumenac Bay and Campbelltown in Canada. Geological Magazine (Decade 3) 7: 15-22. Available from https://biodiversitylibrary.org/page/30521971 [accessed 17 Jul. 2018].

Traquair R.H. 1891. List of the fossil Dipnoi and Ganoidei of Fife and the Lothians. Proceedings of the Royal Society of Edinburgh 17: 385-400. https://doi.org/10.1017/S0370164600007057

Traquair R.H. 1896. The extinct vertebrate animals of the Moray Firth area. In: Harvie-Brown J.A. \& Buckley T.E. (eds) A Vertebrate Fauna of the Moray Basin, Volume II: 235-285. David Douglas, Edinburgh. Available from https://biodiversitylibrary.org/page/43022700 [accessed 29 Aug. 2018].

Traquair R.H. 1900. Report on fossil fishes collected by the Geological Survey of Scotland in the Silurian rocks of the south of Scotland. Transactions of the Royal Society of Edinburgh 39 (3): 827-864. https://doi.org/10.1017/S0080456800035237

Traquair R.H. 1903. The Lower Devonian fishes of Gemünden. Transactions of the Royal Society Edinburgh 40 (4): 723-739. https://doi.org/10.1017/S0080456800034797

Turner S. 1976. Thelodonti (Agnatha). In: Westphal F. (ed.) Fossilium Catalogus I, Animalia, pars 122: 1-35. Dr. W. Junk, The Hague.

Tyler J.C. 1968. A monograph on plectognath fishes of the superfamily Triacanthoidea. Monographs of the Academy of Natural Sciences of Philadelphia 16: 1-364.

Tyler J.C. 1973. A new species of triacanthodid fish (Plectognathi) from the Eocene of Monte Bolca, Italy, representing a new subfamily ancestral to the Triodontidae and the other gymnodonts. Miscellanea Paleontologica. Studi e ricerche sui giacimenti terziari di Bolca II: 127-156.

Tyler J.C. 1998. A new family for a long known but undescribed acanthopterygian fish from the Eocene of Monte Bolca, Italy: Sorbiniperca scheuchzeri gen. \& sp. nov. Eclogae Geologicae Helvetiae 91: 521-540. Available from https://www.e-periodica.ch/cntmng?pid=egh-001:1998:91::660 [accessed 17 Jul. 2018].

Tyler J.C. 2004. Review of the species of the Eocene of Monte Bolca, Italy, fish family Aulorhamphidae, new, related to Gasterosteiformes. Miscellanea Paleontologica n. 7. Studi e ricerche sui giacimenti terziari di Bolca X: 37-54.

Tyler J.C. \& Bannikov A.F. 2002. A new genus and species of deep-bodied perciform fish (Teleostei) from the Eocene of Monte Bolca, Italy, representing a new family, the Zorzinichthyidae, related to the 
caproid- and sorbinipercid-like clades. Miscellanea Paleontologica n. 6. Studi e ricerche sui giacimenti terziari di Bolca IX: 23-35.

Tyler J.C. \& Bannikov A.F. 2005. Massalongius, gen. \& fam. nov., a new clade of acanthuroid fishes (Perciformes, Acanthuroidei) from the Eocene of Monte Bolca, Italy, related to the Zanclidae. Miscellanea Paleontologica n. 8. Studi e ricerche sui giacimenti terziari di Bolca XI: 75-95.

Tyler J.C. \& Bannikov A.F. 2009. Phylogenetic implications of some cranial features of the porcupine pufferfish Pshekhadiodon (Tetraodontiformes, Diodontidae) from the Eocene of the Northern Caucasus. Journal of Ichthyology 49 (9): 703-709. [Voprosy Ikhtiologii 49 (6): 725-731.]

https://doi.org/10.1134/S003294520909001X

Tyler J.C. \& Santini F. 2005. A phylogeny of the fossil and extant zeiform-like fishes, Upper Cretaceous to Recent, with comments on the putative zeomorph clade (Acanthomorpha). Zoologica Scripta 34 (2): 157-175. https://doi.org/10.1111/j.1463-6409.2005.00180.x

Tyler J.C. \& Sorbini L. 1996. New superfamily and three new families of tetraodontiform fishes from the Upper Cretaceous: the earliest and most morphologically primitive plectognaths. Smithsonian Contributions to Paleobiology 82: 1-59. Available from

https://repository.si.edu/bitstream/handle/10088/1998/SCtP-0082-Hi_res.pdf?sequence=1\&isAllowed=y [accessed 17 Jul. 2018].

Tyler J.C. \& Sorbini L. 1998. A new genus and species of primitive triggerfish from the Eocene of Monte Bolca, Italy; the earliest known balistoid (Tetraodontiformes). Miscellanea Paleontologica n. 4. Studi e ricerche sui giacimenti terziari di Bolca VII: 43-65.

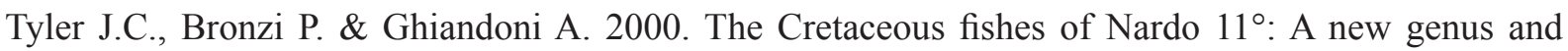
species of Zeiformes, Cretazeus rinaldii, the earliest record for the order. Bollettino del Museo Civico di Storia Naturale di Verona, Geologia Paleontologia Preistoria 24: 11-28.

Underwood C.J. \& Cumbaa S.L. 2010. Chondrichthyans from a Cenomanian (Late Cretaceous) bonebed, Saskatchewan,Canada.Palaeontology53(4):903-944.https://doi.org/10.1111/j.1475-4983.2010.00969.x

Underwood C.J. \& Ward D.J. 2004. Neoselachian sharks and rays from the British Bathonian (Middle Jurassic). Palaeontology 47 (3): 447-501. https://doi.org/10.1111/j.0031-0239.2004.00386.x

Uyeno T. 1967. A Miocene alepisauroid fish of a new family, Polymerichthyidae, from Japan. Bulletin of the National Science Museum, Tokyo 10 (3): 383-394.

Valiukevičius J. \& Burrow C.J. 2005. Diversity of tissues in acanthodians with Nostolepis-type histological structure. Acta Palaeontologica Polonica 50 (3): 635-649.

Available from https://www.app.pan.pl/archive/published/app50/app50-635.pdf [accessed 17 Jul. 2018].

Van der Laan R. 2018. Cumulative Addenda to Family-Group Names in Recent Fishes. Available from http://www.calacademy.org/scientists/catalog-of-fishes-family-group-names/ [accessed 31 May 2018].

Van der Laan R., Eschmeyer W.N. \& Fricke R. 2014. Family-Group Names of Recent Fishes. Zootaxa 3882 (1): 1-230. https://doi.org/10.11646/zootaxa.3882.1.1

Vanhoorne B. 2017. The Interim Register of Marine and Nonmarine Genera. Australian Ocean Biogeographic Information System (OBIS Australia). Checklist Dataset. https://doi.org/10.15468/6tkudz

Vergoossen J.M.J. 1997. Revision of the poracanthodid acanthodians. In: Ivanov A., Wilson M.V.H. \& Zhuravalov A. (eds) Palaeozoic Strata and Fossils of the Eurasian Arctic. Ichthyolith Issues Special Publication 3: 44-46. St. Petersburg. 
Vézina D. 1990. Les Plourdosteidae fam. nov. (Placodermi, Arthrodira) et leurs relations phylétiques au sein des Brachythoraci. Canadian Journal of Earth Science 27: 677-683.

https://doi.org/10.1139/e90-065

Vogt K.C. 1851. Zoologische Briefe, Naturgeschichte der lebenden und untergegangenen Thiere, für Lehrer, höhere Schulen und Gebildete aller Stände, Band II. Literarische Anstalt, Frankfurt am Main. Available from https://www.biodiversitylibrary.org/item/16327 [accessed 17 Jul. 2018].

Voichyshyn V.K. 1999. The new forms of pteraspids (Agnatha, Heterostraci) from Podolian Early Devonian. Vestnik Zologii 33 (3): 47-56. [Author also seen as Voichychyn.]

Voigt E. 1934. Die Fische aus der mitteleozänen Braunkohle des Geiseltales mit besonderer Berücksichtigung der erhaltenen Weichteile. Nova Acta Leopoldina (Neue Folge) 2 (1-2): 21-146.

Vorobyeva E.I. 1967. [A Triassic ceratod from southern Ferghana and some remarks on the systematics and phylogeny of the ceratodontids]. Paleontologicheskii Zhurnal 1967 (4): 102-111. [In Russian, English translation in Paleontological Journal 1967: 80-87; author also seen as Vorobjeva or Vorob'eva or Worobjewa or Worobyeva.]

Vorobyeva E.I. 1975. Formenvielfalt und Verwandtschaftsbeziehungen der Osteolepidida (Crossopterygii, Pisces). Paläontologische Zeitschrift 49 (1/2): 44-55. https://doi.org/10.1007/BF02988065

Vorobyeva E.I. 1977a. Morfologiya i osobennosti evolyutsii kisteperykh ryb [Morphology and evolutionary features of crossopterygian fishes / Morphology and nature of evolution of crossopterygian fishes]. Trudy Paleontologicheskogo Instituta Akademiya Nauk SSSR 163: 1-239. [In Russian.]

Vorobyeva E.I. 1977b. Evolutionary modifications of the teeth structure in the Palaeozoic Crossopterygii. Journal of the Palaeontological Society of India 20 [1975]: 16-20. Available from http://palaeontologicalsociety.in/vol20/v5.pdf [accessed 17 Jul. 2018].

Vorobyeva E.I. 1977c. [The phylogenetic connections of the osteolepiform Crossopterygii and their systematic position]. In: Menner V.V. (ed.) Ocherki po filogenii i sistematike iskopayemykh ryb $i$ beschelyustnykh [Outlines on the Phylogeny and Systematics of Fossil Fishes and Agnathans]: 71-88. Paleontologicheskogo Instituta Akademii Nauka, Moscow. [In Russian.]

Vorobyeva E.I. 2004. [Agnathans and early fishes: subclass Crossopterygii (crossopterygians)]. In: Novitskaya L.I. \& Afanassieva O.B. (eds) Iskopayemyye pozvonochnyye Rossii i sopredel'nykh stran. Beschelyustnyye i drevniye ryby. Spravochnik dlya paleontologov, biologov i geologov [Fossil Vertebrates of Russia and Adjacent Countries. The Reference Book for Paleontologists, Biologists and Geologists]: 272-372. GEOS, Moscow. [In Russian.]

Vorobyeva E.I. \& Lyarskaya L.A. 1968. Ostatki kisteperykh i dvoyakodyshashchikh ryb iz amatskikh sloev Latvii i ikh zahkoronenie [Remains of crossopterygians and dipnoans from the Amata Beds in Latvia and their burial conditions]. In: Obruchev D.V. (ed.) Ocherki po filogenii i sistematike iskopayemykh ryb $i$ beschelyustnykh [Outlines on the phylogeny and systematics of the fossil fishes and agnathans]: 71-86. Nauka, Moscow. [In Russian.]

Vullo R., Guinot G. \& Barbe G. 2016. The first articulated specimen of the Cretaceous mackerel shark Haimirichia amonensis gen. nov. (Haimirichiidae fam. nov.) reveals a novel ecomorphological adaptation within the Lamniformes (Elasmobranchii). Journal of Systematic Palaeontology 14 (12): 1003-1024. https://doi.org/10.1080/14772019.2015.1137983

Wade R.T. 1932. Preliminary note on Macroaethes brookvalei, representing a new family of chondrostean fishes, the Pholidopleuridae. Annals and Magazine of Natural History (series 10) 9 (53): 473-475. https://doi.org/10.1080/00222933208673520 
Wade R.T. 1935. The Triassic Fishes of Brookvale, New South Wales. British Museum (Natural History), London.

Wade R.T. 1941a. Australian Triassic Fishes. I. The Triassic fishes of St. Peter's, Sydney, New South Wales. Journal and Proceedings of the Royal Society of New South Wales 74 [for 1940]: 377-388. Available from http://biodiversitylibrary.org/page/46187078 [accessed 17 Jul. 2018].

Wade R.T. 1941b. The Jurassic fishes of New South Wales. Journal and Proceedings of the Royal Society of New South Wales 75: 71-84. Available from http://biodiversitylibrary.org/page/46123852 [accessed 17 Jul. 2018].

Wagner J.A. 1860a. Vergleichung der urweltlichen Fauna des lithographischen Schiefers von Cirin mit der der gleichnamigen Ablagerungen im fränkischen Jura (Fortsetzung). Gelehrte Anzeigen der königlichen bayerischen Akademie der Wissenschaften, München (49): 393-400. [Published 2 May.]

Wagner J.A. 1860b. Vergleichung der urweltlichen Fauna des lithographischen Schiefers von Cirin mit der der gleichnamigen Ablagerungen im fränkischen Jura (Fortsetzung). Gelehrte Anzeigen der königlichen bayerischen Akademie der Wissenschaften, München (50): 401-408. [Published 5 May.]

Wagner J.A. 1863. Monographie der fossilen Fische aus den lithographischen Schiefern Bayern's II. Abhandlungen der Mathematisch-Physikalischen Klasse der Königlich Bayerischen Akademie der Wissenschaften 9: 611-748.

Available from https://biodiversitylibrary.org/page/35523290 [accessed 29 Aug. 2018].

Waldman M. 1971. Fish from the Freshwater Lower Cretaceous of Victoria, Australia, with Comments on the Palaeo-Environment. Special papers in palaeontology 9. Palaeontological Association, London. Available from https://www.palass.org/sites/default/files/media/publications/special_papers_in_ palaeontology/number_9/spp9_pp1-124.pdf [accessed 16 Sep. 2018].

Wang C.-C. 1979. A new family of Arthrodira from Yunnan, China. Vertebrata PalAsiatica 17 (3): 179-188. [In Chinese, with English summary.]

Available from http://www.ivpp.cas.cn/cbw/gjzdwxb/xbwzxz/200904/W020090813375924994740.pdf [accessed 17 Jul. 2018].

Wang J.-Q. \& Wang N.-Z. 1992. Early Devonian Galeaspid Agnatha from Southeast of Yunnan, China. Vertebrata PalAsiatica 30 (3): 185-194. [In Chinese, with English summary.]

Available from http://www.ivpp.cas.cn/cbw/gjzdwxb/xbwzxz/200812/W020090813370921464048.pdf [accessed 17 Jul. 2018].

Wang J.-Q. \& Zhu M. 1994. Zhaotongaspis janvieri gen. et sp. nov., a galeaspid from Early Devonian of Zhaotong, Northeastern Yunnan. Vertebrata PalAsiatica 32 (4): 231-243.

Available from http:/www.ivpp.cas.cn/cbw/gjzdwxb/xbwzxz/200812/W020090813370331847077.pdf [accessed 17 Jul. 2018].

Wang S.-T. 1987. A new antiarch from the Early Devonian of Guangxi. Vertebrata PalAsiatica 25 (2): 81-90. [In Chinese, with English summary.]

Available from http://www.ivpp.cas.cn/cbw/gjzdwxb/xbwzxz/200903/P020100326345549658451.pdf [accessed 17 Jul. 2018].

Wängsjo G. 1952. The Downtonian and Devonian vertebrates of Spitsbergen, IX. Morphologic and systematic studies of the Spitsbergen Cephalaspids. A. Text, B. Plates. Norsk Polarinstitutt Skrifter (97): 1-653. Available from http://hdl.handle.net/11250/173537 [accessed 27 Jul. 2018].

Weiler W. 1935. Ergebnisse der Forschungsreisen Prof. E. Stromers in den Wüsten Ägyptens, II. Wirbeltierreste der Baharîje-Stufe (unterstes Cenoman). 16. Neue Untersuchungen an den Fischresten. Abhandlungen der Bayerischen Akademie der Wissenschaften Mathematisch-naturwissenschaftliche Abteilung (N.F.) 32: 1-57. 
Wendruff A.J. \& Wilson M.V.H. 2012. A fork-tailed coelacanth, Rebellatrix divaricerca, gen. et sp. nov. (Actinistia, Rebellatricidae, fam. nov.), from the Lower Triassic of western Canada. Journal of Vertebrate Paleontology 32 (3): 499-511. https://doi.org/10.1080/02724634.2012.657317

Werner C. 1989. Die Elasmobranchier-Fauna des Gebel Dist Member der Bahariya Formation (Obercenoman) der Oase Bahariya, Ägypten. Palaeo Ichthyologica 5: 1-112.

Westheide W. \& Rieger G. (eds) 2015. Spezielle Zoologie. Teil 2: Wirbel- oder Schädeltiere, $3^{\text {rd }}$ edition. Springer Spectrum.

Westoll T.S. 1944. The Haplolepidae, a new family of Late Carboniferous bony fishes, a study in taxonomy and evolution. Bulletin of the American Museum of Natural History 83: 1-121. Available from http://hdl.handle.net/2246/388 [accessed 17 Jul. 2018].

White E.G. 1936. A classification and phylogeny of the elasmobranch fishes. American Museum Novitates (837): 1-16. Available from http://hdl.handle.net/2246/4163 [accessed 17 Jul. 2018].

White E.I. 1935a. Fossil fishes of Sokoto Province. Bulletin of the Geological Survey of Nigeria No. 14: 1-77. [Sometimes dated 1934.]

White E.I. 1935b. The ostracoderm Pteraspis Kner and the relationships of the agnathous vertebrates. Philosophical Transactions of the Royal Society B 225 (527): 381-457. Available from http://www.jstor.org/stable/92279 [accessed 17 Jul. 2018].

White E.I. 1939. A new type of palaeoniscoid fish, with remarks on the evolution of the Actinopterygian pectoral fins. Proceedings of the Zoological Society of London 109 (1): 41-61.

https://doi.org/10.1111/j.1469-7998.1939.tb00023.x

White E.I. 1946a. Jamoytius kerwoodi, a new Chordate from the Silurian of Lanarkshire. Geological Magazine 83 (2): 89-97. https://doi.org/10.1017/S0016756800082856

White E.I. 1946b. The genus Phialaspis and the "Psammosteus Limestones". The Quarterly Journal of the Geological Society of London 101 (3/4) [for 1945]: 207-242.

https://doi.org/10.1144/GSL.JGS.1945.101.01-04.10

White E.I. 1952. Australian Arthrodires. Bulletin of the British Museum (Natural History), Geology 1 (9): 249-304. Available from https://www.biodiversitylibrary.org/page/36367846 [accessed 27 Jul. 2018].

White E.I. 1956. XIII Pisces. Zoological Record 91: 66.

White E.I. 1961. The Old Red Sandstone of Brown Clee Hill and the adjacent area. Part II. Palaeontology. Bulletin of the British Museum (Natural History), Geology 5 (7): 243-310. Available from https://biodiversitylibrary.org/page/36368328 [accessed 29 Aug. 2018].

White E.I. 1968. Devonian fishes of the Mawson-Mulock area, Victoria Land, Antarctica. Scientific Report of the Trans-Antarctic Expedition 1955-1958, Geology (16): 1-26.

White E.I. 1978. The larger arthrodiran fishes from the area of the Burrinjuck Dam, N.S.W. Journal of Zoology 34 (2): 149-262. https://doi.org/10.1111/j.1096-3642.1978.tb00374.x

Whitley G.P. 1933. New names for fossil fishes. Copeia 1933 (3): 146.

Available from http://www.jstor.org/stable/1436242 [accessed 27 Jul. 2018].

Whitley G.P. 1940. The Nomenclator Zoologicus and some new fish names. The Australian Naturalist 10 (7): 241-243. 
Whitley G.P. 1950. New fish names. Proceedings of the Royal Zoological Society of New South Wales for 1948-49: 44. Available from https://www.biodiversitylibrary.org/page/38773495 [accessed 17 Jul. 2018].

Whitley G.P. 1951. New fish names and records. Proceedings of the Royal Zoological Society of New South Wales, for 1949-50: 61-68. Available from https://www.biodiversitylibrary.org/page/38767730 [accessed 17 Jul. 2018].

Whitley G.P. 1976. More fish genera scrutinized. Australian Zoologist 19 (1): 45-50. Available from https://biodiversitylibrary.org/page/38968551 [accessed 29 Aug. 2018].

Wiley E.O. \& Johnson D.G. 2010. A teleost classification based on monophyletic groups. In: Nelson J., Schultze H.-P. \& Wilson M.V.H. (eds) Origin and Phylogenetic Interrelationships of Teleosts: 123-182. Verlag Dr. Friedrich Pfeil, Munich.

Williams M.E. 1979. The "Cladodont Level" Sharks of the Pennsylvanian Black Shales of Central North America. Unpublished dissertation, University of Kansas, Lawrence, USA.

Williams M.E. 1985. The "cladodont level" sharks of the Pennsylvanian black shales of central North America. Palaeontographica (Abteilung A: Paläozoologie-Stratigraphie) 190 (3-6): 83-158.

Wilson M.V.H. \& Caldwell M.W. 1998. The Furcacaudiformes, a new order of jawless vertebrates with thelodont scales, based on articulated Silurian and Devonian fossils from northern Canada. Journal of Vertebrate Paleontology 18 (1): 10-29. Available from http://www.jstor.org/stable/4523870 [accessed 17 Jul. 2018].

Wilson M.V.H. \& Märss T. 2009. Thelodont phylogeny revisited, with inclusion of key scale-based taxa. Estonian Journal of Earth Sciences 58 (4): 297-310. Available from

http://www.kirj.ee/public/Estonian_Journal_of_Earth_Sciences/2009/issue_4/earth-2009-4-297-310.pdf [accessed 17 Jul. 2018].

Wilson M.V.H. \& Williams R.R.G. 1992. Phylogenetic, biogeographic, and ecological significance of early fossil records of North American freshwater teleostean fishes. In: Mayden R.L. (ed.) Systematics, Historical Ecology, and North American Freshwater Fishes: 224-244. Stanford University Press, Stanford.

Winterbottom R. 1974. The familial phylogeny of the Tetraodontiformes (Acanthopterygii: Pisces) as evidenced by their comparative myology. Smithsonian Contributions to Zoology 155: 1-201.

Available from

https://repository.si.edu/bitstream/handle/10088/5246/SCtZ-0155-Hi_res.pdf?sequence=1\&isAllowed=y [accessed 17 Jul. 2018].

Woodward A.S. 1889a. Catalogue of the Fossil Fishes in the British Museum (Natural History), Part I. British Museum (Natural History), London.

Available from https://www.biodiversitylibrary.org/item/211828 [accessed 17 Jul. 2018].

WoodwardA.S. 1889b. On the Myriacanthidae, an extinct family of chimaroid fishes. Annals and Magazine of Natural History (series 6) 4 (22): 275-280. [October] https://doi.org/10.1080/00222938909460526

Woodward A.S. 1889c. Notes on some new and little-known British Jurassic fishes [Abstract.]. Annals and Magazine of Natural History (series 6) 4 (23): 405-407. https://doi.org/10.1080/00222938909460552

Woodward A.S. 1890a. The fossil fishes of the Hawkesbury Series at Gosford [Abstract.]. Annals and Magazine of Natural History (series 6) 6 (35): 423-424. https://doi.org/10.1080/00222939008694061

Woodward A.S. 1890b. The fossil fishes of the Hawkesbury Series at Gosford. Memoirs of the Geological Survey of New South Wales, Palaeontology 4: 1-57.

Available from https://biodiversitylibrary.org/page/49593502 [accessed 29 Aug. 2018]. 
Woodward A.S. 1891a. Catalogue of the Fossil Fishes in the British Museum (Natural History) Part II. British Museum (Natural History), London.

Available from https://www.biodiversitylibrary.org/item/125733 [accessed 17 Jul. 2018].

Woodward A.S. 1891b. Reviews. Armoured Palaeozoic sharks. Geological Magazine (Decade 3) 8: 422-425. Available from https://biodiversitylibrary.org/page/30545808 [accessed 17 Jul. 2018].

Woodward A.S. 1891c. The Devonian fish-fauna of Spitzbergen. Annals and Magazine of Natural History (series 6) 8 (43): 1-15. https://doi.org/10.1080/00222939109460384

Woodward A.S. 1895. Catalogue of the Fossil Fishes in the British Museum (Natural History), Part III. British Museum (Natural History), London.

Available from https://www.biodiversitylibrary.org/item/125967 [accessed 17 Jul. 2018].

Woodward A.S. 1900. On a new Ostracoderm (Euphanerops longaevus) from the Upper Devonian of Scaumenac Bay, Province of Quebec, Canada. Annals and Magazine of Natural History (series 7) 5 (29): 416-419. [After 7 October and before December.] https://doi.org/10.1080/00222930008678308

Woodward A.S. 1901. Catalogue of Fossil Fishes in the British Museum (Natural History), Part IV. British Museum (Natural History), London. [after 7 October and before December]

Available from https://www.biodiversitylibrary.org/item/213418 [accessed 17 Jul. 2018].

Woodward A.S. 1902. The fossil fishes of the English Chalk. Part 1. Palaeontographical Society Monograph 56: 1-56. Available from https://biodiversitylibrary.org/page/36960670 [accessed 17 Jul. 2018].

Woodward A.S. 1903. The fossil fishes of the English Chalk. Part 2. Palaeontographical Society Monograph 57: 57-96. Available from https://biodiversitylibrary.org/page/36966028 [accessed 17 Jul. 2018].

Woodward A.S. 1906. On a Carboniferous Fish Fauna from the Mansfield District, Victoria. Memoirs of the National Museum, Melbourne 1: 1-32. https://doi.org/10.24199/j.mmv.1906.1.01

Woodward A.S. 1912. The fossil fishes of the English Chalk. Part 7 [With title-page and index.]. Palaeontographical Society Monograph 65: 225-264.

Available from https://biodiversitylibrary.org/page/36923116 [accessed 17 Jul. 2018].

Woodward A.S. 1919. On two new elasmobranch fishes (Crossorhinus jurassicus, sp. nov., and Protospinax annectans, gen. et sp. nov.) from the Upper Jurassic lithographic stone of Bavaria. Proceedings of the Zoological Society of London: 231-235.

https://doi.org/10.1111/j.1096-3642.1918.tb02093.x

Woodward A.S. 1931. On Urosthenes, a fossil fish from the Upper Coal Measures of Lithgow, New South Wales. Annals and Magazine of Natural History (series 10) 8 (46): 365-367. https://doi.org/10.1080/00222933108673406

Woodward A.S. 1932. Fishes. In: Zittel K.A. (ed.) Text-Book of Palaeontology, Volume 2. $2^{\text {nd }}$ English edition. Macmillan, London.

Woodward A.S. \& Sherborn C.D. 1890. A Catalogue of British Fossil Vertebrata. Dulau and Co, London. https://doi.org/10.5962/bhl.title.30421

Wu F.X., Chang M.-M., Sun Y.L. \& Xu G.H. 2013. A new saurichthyiform (Actinopterygii) with a crushing feeding mechanism from the Middle Triassic of Guizhou (China). PLoS One 8 (12): e81010. https://doi.org/10.1371/journal.pone.0081010 
Xu G.-H. \& Zhao L.-J. 2016. A Middle Triassic stem-neopterygian fish from China shows remarkable secondary sexual characteristics. Science Bulletin 61 (4): 338-344.

https://doi.org/10.1007/s11434-016-1007-0

Yabumoto Y. 1994. Early Cretaceous freshwater fish fauna in Kyushu, Japan. Bulletin of the Kitakyushu Museum of Natural History 13:107-254.

Available from http://www.kmnh.jp/wp-content/themes/kmnh_jp/images/pdf/13-107-E-Yabumoto.pdf [accessed 16 Sep. 2018].

Yakovlev V.N. 1962. Ryby ostryada Pholidophoriformes yuri Karatau [Jurassic fishes of the order Pholidophoriformes from Karatau]. Paleontologicheskii Zhurnal 1962 (3): 90-101. [In Russian, author also seen as Jakovlev or Yakowlew]

Young G.C. 1986. The relationships of placoderm fishes. Zoological Journal of the Linnean Society 88 (1): 1-57. https://doi.org/10.1111/j.1096-3642.1986.tb00876.x

Young G.C. 1991. The first armoured agnathan vertebrates from the Devonian of Australia. In: Chang M.-M., Liu Y.-H. \& Zhang G.-R. (eds) Early Vertebrates and Related Problems of Evolutionary Biology: 67-85. Science Press, Beijing.

Young G.C. 2008. Relationships of tristichopterids (osteolepiform lobe-finned fishes) from the MiddleLate Devonian of East Gondwana. Alcheringa 32 (3): 321-336.

https://doi.org/10.1080/03115510802104368

Young G.C. 2010. A new antiarch (placoderm fish: Devonian) from the south coast of New South Wales, Australia. In: Elliott D.K., Maisey J.G., Yu X. \& Miao D. (eds) Morphology, Phylogeny and Paleobiogeography of Fossil Fishes (Honoring Meemann Chang): 85-100. Verlag Dr. Friedrich Pfeil, Munich.

Young G.C. \& Goujet D. 2003. Devonian fish remains from the Dulcie Sandstone and Cravens Peak Beds, Georgina Basin, central Australia. Records of the Western Australian Museum Supplement 65: 1-85. https://doi.org/10.18195/issn.0313-122x.65.2003.001-085

Young G.C., Long J.A. \& Ritchie A.R. 1992. Crossopterygian fishes from the Devonian of Antarctica: systematics, relationships and biogeographic significance. Records of the Australian Museum Supplement 14: 1-77. https://doi.org/10.3853/j.0812-7387.14.1992.90

Young J. 1866. On the affinities of Platysomus and allied genera. The Quarterly Journal of the Geological Society of London 22: 301-317. https://doi.org/10.1144/GSL.JGS.1866.022.01-02.22

Zajíc J. 1995. Some consequences of recent investigations on the family Acanthodidae Huxley, 1861. Geobios 28 (Supplement 2): 167-169. https://doi.org/10.1016/S0016-6995(95)80107-3

Zangerl R. 1969. Bandringa rayi, a new ctenacanthoid shark form the Pennsylvanian Essex fauna of Illinois. Fieldiana Geology 12 (10): 157-169.

Available from https://www.biodiversitylibrary.org/page/4254470 [accessed 27 Jul. 2018].

Zangerl R. 1979. New Chondrichthyes from the Mazon Creek fauna (Pennsylvanian) of Illinois. In: Nitecki M.H. (ed.) Mazon Creek Fossils: 449-500. Academic Press, New York.

Zangerl R. 1981. Chondrichthyes I: Paleozoic Elasmobranchii. In: Schultze H.-P. (ed.) Handbook of Paleoichthyology Volume 3A: 1-115. Gustav Fischer Verlag, Stuttgart / New York.

Zangerl R. 1990. Two new stethacanthid sharks (Stethacanthidae, Symmoriida) from the Pennsylvanian of Indiana, U.S.A. Palaeontographica (Abteilung A: Paläozoologie-Stratigraphie) 213 (1-6): 115-141.

Zangerl R. \& Case G.R. 1973. Iniopterygia: a new order of Chondrichthyan fishes from the Pennsylvanian of North America. Fieldiana Geology, Memoir 6: 1-67. https://doi.org/10.5962/bhl.title.5158 
Zhang G.-R. 1978a. [Early Devonian antiarchs from Cuifengshan, Yunnan.] In: Symposium on the Devonian System of South China 1974, Institute of Geology and Mineral Resources, Chinese Academy of Geological Sciences: 292-297. Geological Publishing House, Beijing. [In Chinese, author also seen as Chang.]

Zhang G.-R. 1978b. The Antiarchs from the Early Devonian of Yunnan. Vertebrata PalAsiatica 16 (3): 147-186. [In Chinese, with English summary.]

Available from http://www.ivpp.cas.cn/cbw/gjzdwxb/xbwzxz/200904/P020100309309425560745.pdf [accessed 17 Jul. 2018].

Zhang G.-R. 1984. New form of Antiarchi with primitive brachial process from Early Devonian of Yunnan. Vertebrata PalAsiatica 22 (2): 81-91. [In Chinese, with English summary.]

Available from http://www.ivpp.cas.cn/cbw/gjzdwxb/xbwzxz/200903/W020090813374488433591.pdf [accessed 17 Jul. 2018].

Zhang G.-R. \& Liu Y.G. 1991. A new antiarch from the Upper Devonian of Jiangxi, China. In: Chang M.-M., Liu Y.-H. \& Zhang G.-R. (eds) Early Vertebrates and Related Problems of Evolutionary Biology: 195-212. Science Press, Beijing.

Zhang G.-R., Wang S.-T., Wang J.-Q., Wang N.-Z. \& Zhu M. 2010. A basal antiarch (placoderm fish) from the Silurian of Qujing, Yunnan, China. Palaeoworld 19 (1-2): 129-135.

https://doi.org/10.1016/j.palwor.2009.11.006

Zhelezko V.I. 1989. [Phylogenesis of lamnoid sharks of the Palaeogene and their significance for zonal stratigraphy]. In: Kruchinina N.V.\& Modzalevskaya T.L. (eds) Filogeneticheskie aspekty paleontologii. Tezisy dokladov 35-01 sessii Vsesoyuznogo paleontologicheskogo obshchestva [Phylogenetic Aspects of Palaeontology. Extended Abstracts.]: 16-17. Academy of Sciences USSR, All-Union Palaeontological Society, Leningrad. [In Russian.]

Zhelezko V.I. \& Glickman L.S. 1971. [About Cenomanian deposits of Western Kazakhstan and some Cretaceous sclerophagous sharks]. In: Problemy geologii Zapadnogo Kazahstana Alma-Ata (k 60-letiyu akademika Aleksandra Leonidovicha Yanshina): 179-188. Nauka Kazakh SSR Publishers, Alma-Ata. [In Russian.]

Zhu M. 1998. Early Silurian sinacanths (Chondrichthyes) from China. Paleontology 41 (1): 157-171. Available from https://www.palass.org/sites/default/files/media/publications/palaeontology/volume_41/ vol41_part1_pp157-171.pdf [accessed 17 Jul. 2018].

Zhu M. \& Gai Z.-K. 2006. Phylogenetic relationships of galeaspids (Agnatha). Vertebrata PalAsiatica 44 (1): 1-27.

Available from http://www.ivpp.cas.cn/cbw/gjzdwxb/xbwzxz/200810/W020090813368820232854.pdf [accessed 17 Jul. 2018].

Zidek J. 1993. Acanthodii. In: Benton M.J. (ed.) The Fossil Record 2: 589-592. Chapman \& Hall, London.

Zittel K.A. von 1887-1890. Handbuch der Palaeontologie. I. Abtheilung Palaeozoologie III. Band Vertebrata (Pisces, Amphibia, Reptilia, Aves). Oldenbourg Verlag, Munich.

Available from https://www.biodiversitylibrary.org/item/124860 [accessed 17 Jul. 2018].

Zittel K.A. von 1895. Grundzüge der Palaeontologie (Palaeozoologie): 510-603. Oldenbourg Verlag, Munich / Leipzig.

Zittel K.A. von 1902. Text-book of Palaeontology, Volume 2. MacMillan and Co, London. [Translated and edited by C.R. Eastman.] Available from https://www.biodiversitylibrary.org/item/125342 [accessed 17 Jul. 2018]. 
Zittel K.A. von 1911. Grundzüge der Paläontologie (Paläozoologie). II. Abteilung: Vertebrata. 2. Auflage, neu bearbeitet von F. Broili, E. Koken \& M. Schlosser: 1-142. Oldenbourg Verlag, Munich. Available from https://www.biodiversitylibrary.org/item/125555 [accessed 17 Jul. 2018].

Zittel K.A. von 1918. Grundzüge der Paläontologie (Paläozoologie). II. Abteilung: Vertebrata. $3^{\text {rd }}$ edition. F. Broili und M. Schlosser. Oldenbourg Verlag, Munich / Berlin.

Zych W. 1931. Fauna ryb Dewonu i Downtonu Podola, Pteraspidomorphi: Heterostraci. Czesc (Lwów) IA: 1-91. [In Polish.]

Manuscript received: 27 September 2017

Manuscript accepted: 11 June 2018

Published on: 11 October 2018

Topic editor: Christian de Muizon

Desk editor: Kristiaan Hoedemakers

Printed versions of all papers are also deposited in the libraries of the institutes that are members of the EJT consortium: Muséum national d'Histoire naturelle, Paris, France; Botanic Garden Meise, Belgium; Royal Museum for Central Africa, Tervuren, Belgium; Natural History Museum, London, United Kingdom; Royal Belgian Institute of Natural Sciences, Brussels, Belgium; Natural History Museum of Denmark, Copenhagen, Denmark; Naturalis Biodiversity Center, Leiden, the Netherlands; Museo Nacional de Ciencias Naturales-CSIC, Madrid, Spain; Real Jardín Botánico de Madrid CSIC, Spain; Zoological Research Museum Alexander Koenig, Bonn, Germany. 
Index [Recent fish families in bold]

A

Acanthaspidae

Acanthodermidae

Acanthodidae

Acanthodiens

Acanthoëssidae

Acanthonemidae

Acanthopsides

Acanthorhinidae

Acanthuridae

Acentrophoridae

Aceraspidae

Acestrorhynchidae

Achanarellidae

Acheilognathidae

Achiridae

Achiropsettidae

Acipenseridae

Acritolepidae

Acrodini

Acrodontidae

Acrolepididae

Acrolepidae .

Acropholidae

Acropomatidae

Acrotomaspididae

Actinolepidae

Actinolepididae

Aculeolidae

Adrianichthyidae

Aeduellidae

Aesopichthyidae

Aestiaspidinae

Aetheodontidae

Aetheolepidae

Aetobatidae

Agaleidae

Agassizodontidae

Aggeraspididae

Aglyptorhynchinae

Agonidae

Ailiidae

Aipichthyidae

Aipichthyoididae.

Akysidae.

Albulidae

Alepisauridae
Alepocephalidae $\quad$............................64 64

Alestidae....................................66

Alloberycinae .............................. 74

Allothrissopidae .............................60 60

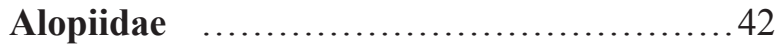

Altholepididae............................... 34

Amarsipidae ................................74

Ambassidae ............................... 78

Amblycipitidae .............................6 67

Amblyopsidae ..............................72

Amblypteridae.................................49

Americaspidae............................... 18

Amiidae ................................. 57

Amiopsinae ................................ 57

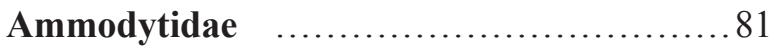

Amphiaspididae .............................17

Amphicentridae .............................51

Amphiliidae ..............................67

Amphistiidae ...............................78

Anabantidae ............................... 76

Anablepidae ............................... 80

Anacanthobatidae ........................... 45

Anachronistidae ...............................40 40

Anacoracidae ................................. 42

Anaethalionidae ..............................61

Anarhichadidae .............................. 86

Anchariidae ................................67 67

Anchipteraspididae ............................ 18

Anchipteraspidinae ......................... 18

Ancylostylidae ..............................65

Andinichthyidae .............................66

Andreolepididae ..............................46

Anenchelini ................................. 75

Angaralepididae ................................21

Angaralepidinae ..............................21

Angaraspididae ...............................17

Anglaspidae................................... 18

Anguillavidae ...............................61

Anguillidae...................................6 62

Anguilloididae.................................6 62

Angustidontidae ............................... 15

Ankylophoridae ...............................59

Anogmiidae.....................................60 60

Anomalopidae ............................73

Anomotodontidae ............................42

Anoplogastridae .......................... 73

Anoplopomatidae .......................... 86

Anostomidae ...............................66 
Antarctaspidae

Antarctaspididae

Antarctilamnidae...

Antennariidae

Apalolepididae

Apateopholidae

Aphaniidae

Aphataspididae

Aphnelepidae

Aphredoderidae.

Apistidae

Aploactinidae

Aplocheilidae

Aplodactylidae

Apogonidae

Apsopelicidae

Apteronotidae

Apulichthyidae

Aracanidae

Arambourgellidae

Arandaspididae

Araripichthyidae

Archaeinae

Archaeobatidae

Archaeolamnidae

Archaeomaenidae

Archaeozeidae

Archipelepididae ...

Arctaspididae

Arctolepidae

Arctolepididae

Arganodontidae

Argentinidae

Arhynchobatidae

Ariaspididae

Ariidae

Ariommatidae

Armigatidae.

Arripidae

Artedidraconidae

Arthropteridae

Asanoinae

Asarotidae

Ascalaboidae

Aseraspididae

Asianthidae

Asiatoceratodontidae

Asineopidae

Askerichthyinae

Asperaspididae
Aspidorhynchidae

Aspidorhynchoidei ...........................57

Aspidosteidae ...............................19

Aspredinidae .............................67

Astephidae .................................67

Astephinae .................................67

Asterodermini $\quad$..............................44

Asterodontidae ..............................54

Asterolepidae ................................27

Asterolepididae .............................27

Asterosteidae ................................27

Astraspidae ...................................16

Astraspididae ...............................16

Astroblepidae ............................67

Ateleaspidae ...............................22

Ateleaspididae $\quad$..............................22

Ateleopodidae .............................69

Atherinidae ................................79

Atherinopsidae ............................79

Atherionidae ................................79

Atherstoniidae .............................46

Auchenipteridae ..........................67

Auchenoglanididae ........................67

Auchenosteidae ................................32

Aulacosteidae .................................29

Aulolepidae ..................................71

Aulopidae ................................... 70

Aulorhamphidae ............................ 75

Aulorhynchidae $\quad$........................... 86

Aulostomidae ............................. 75

Australosomidae ...........................54

Austroglanididae ...........................67

Avitoplectidae ..............................83

Axinaspididae ...............................24

Aztecodontidae ...............................35

\section{B}

Bachmanniidae ...............................66

Badidae ................................... 77

Bagridae ..................................67

Bajaichthyidae $\quad$..............................71

Balistidae ................................. 83

Balitoridae ..............................65

Balkariidae .................................... 83

Bananogmiidae ..............................61

Bandringidae .................................34

Banjosidae ................................. 84

Barbourisiidae ...............................73

Barbuccidae ...............................65 
VAN DER LAAN R., Family-group names of fossil fishes

Barlowodidae

Bassanichthyinae

Bassaniinae

Bathyclupeidae

Bathydraconidae

Bathygadidae

Bathylagidae

Bathymasteridae

Bathysauridae

Bathysauroididae

Bathysauropsidae

Batrachoididae

Bedotiidae

Beerichthyidae

Belantseidae

Belemnobatidae

Belgicaspinae

Belonidae

Belonorhynchidae

Bembridae

Bembropidae

Benneviaspididae

Benneviaspinae

Bernardichthyidae

Berycidae

Berycopsidae

Birgeriidae

Birkeniidae

Blenniidae

Blochiidae

Blochioidei

Blochioides

Bobasatraniidae

Bolcabalistidae

Bolcyridae

Boothialepididae

Boothiaspidinae

Boreaspidae

Boreaspididae

Boreiohydriidae

Boreolepidae

Boreolepididae

Boreosomidae

Bothidae

Bothriolepididae

Botiidae

Bovichtidae

Brachaeluridae

Brachionichthyidae

Brachyacanthidae

\section{.21}

.85

. .85

. .84

.85

.72

.68

. .86

.70

.70

..70

.74

.79

.79

.81

. .37

.44

.18

.79

.50

.85

.85

. .24

.24

. .68

.73

.73

47

.19

80

.77

.77

.77

.50

.83

62

20

18

.24

.24

73

47

47

51

78

28

65

85

41

82

.34
Brachydegmidae $\quad$.........................54

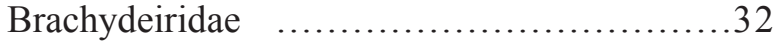

Brachydiridae ............................... 32

Brachyrhizodontidae ......................46

Bramidae .............................. 74

Braunosteidae ..............................32

Brazilichthyidae ...........................47

Bregmacerotidae .........................72

Brembodontidae ............................53

Brindabellaspidae ........................26

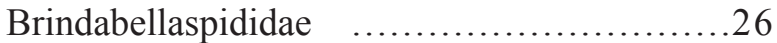

Brochoadmonidae ............................34

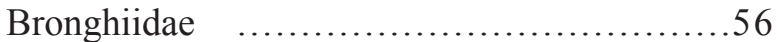

Brookvaliidae ................................50

Brychaetidae ...........................63

Bryconidae ................................66

Buchanosteidae ..............................31

Bulbocanthidae ..............................32

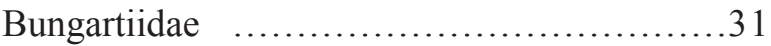

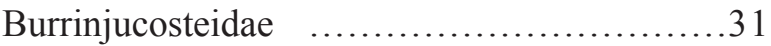

Butidae ...................................76

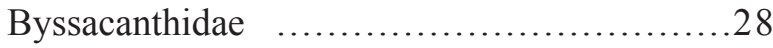

Bythitidae ............................. 74

\section{C}

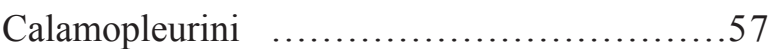

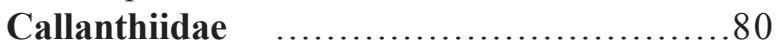

Callichthyidae ............................67

Callionymidae ............................75

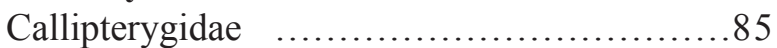

Callipurbeckiidae ........................56

Callorhinchidae $\quad$...........................39

Camuropiscidae ...............................31

Canobiidae ...................................47

Canowindridae ..............................92

Caproberycidae .............................74

Caproberycinae ..............................74

Caproidae .................................82

Caprovesposidae $\quad$...........................81

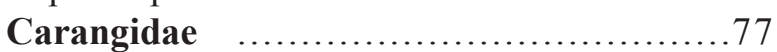

Carangodidae ..................................74

Carbovelidae .................................44 47

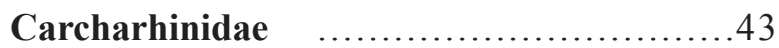

Carchariidae ..............................42

Cardabiodontidae ..........................442

Cardipeltidae ...............................16

Caristiidae .................................74

Carnevalellinae .............................882

Caseodontidea ...............................37

Caseodontoidea ................................37 
Catervariolidae

Catopteridae

Catostomidae

Caturidae

Caucasichthyidae

Caulophrynidae

Cebidichthyidae

Célacanthes

Centrarchidae

Centriscidae

Centrobatidae

Centrogenyidae

Centrolepididae

Centrolophidae

Centrophoridae

Centrophrynidae

Centropomidae

Cephalaspides

Cephalaspidides

Cephaloxenidae

Cepolidae

Ceraspidae

Ceratiidae

Ceratodontidae

Ceratodontina

Ceratolepichthyinae

Ceratolepidae

Cetomimidae

Cetopsidae

Cetorhinidae

Chacidae

Chaenopsidae

Chaetodontidae

Chalceidae

Champsodontidae ........................ 84

Chanidae

Channichthyidae ........................... 85

Channidae

Chanoididae

Characidae

Charitosomidae

Chaudhuriidae

Chaunacidae

Cheilodactylidae

Cheimarrichthyidae

Cheiracanthidae

Cheiracanthoidei

Cheirodidae

Cheirolepiden

Cheirolepididae
.59

. .50

.65

.57

.80

.82

.86

.87

.884

.75

.44

.80

.47

.74

.43

.82

.77

.22

.22

.54

.82

.28

.82

.90

.90

.28

.27

.73

.67

.42

67

80

.81

66

.84

65

Cheirothricidae

Chelonichthyda

Chiasmodontidae ...........................74

Chichiidae .................................51

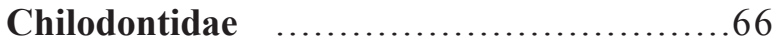

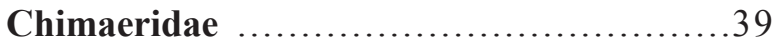

Chimaeropsidae ...............................39

Chirocentridae .............................64

Chirodipteridae ...............................89

Chirodontidae .................................50

Chironemidae ............................ 84

Chirothricidae (............................69

Chlamydoselachidae (.....................44

Chlopsidae ................................62

Chlorophthalmidae ..........................70

Chondrenchelyidae (.........................38

Chondrosteidae ...............................52

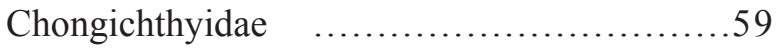

Chrysolepididae ..............................991

Chuchinolepidae .........................27

Chuchinolepididae .........................27

Chuhsiungichthiidae ........................60

Chuhsiungichthyidae .......................60

Chungkingichthyidae ........................51

Cichlidae .................................. 78

Cimolichthyidae .............................70

Cirrhitidae ............................... 84

Citharidae ................................. 78

Citharinidae ...............................66

Cladocyclidae ................................60

Cladodini ......................................36

Cladoselachidae ...............................35

Clariidae ..................................67

Claroteidae .............................67

Cleithrolepidae ............................54

Cleithrolepididae ...........................54

Climatiidae ...................................... 34

Clinidae .................................... 80

Clupavidae .................................64

Clupeidae ................................64

Cobitidae ...................................65

Cobitopsidae ................................79

Coccocephalichthyidae ......................47

Coccodermidae ..............................87

Coccodontidae ...............................53

Coccolepidae ................................46

Cocconiscidae ...............................47

Coccosteida ................................. 31

Coccosteidae ..................................31

Cochliodontidae .............................39 
VAN DER LAAN R., Family-group names of fossil fishes

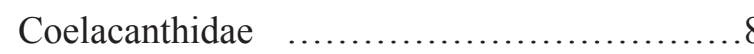

Coelolepiden ................................2

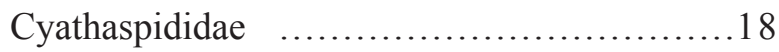

Colobodontidae

Cyclobatidae ....

$.44,45$

Commentryidae

Cyclodiscaspidae

.25

.47

Conchodontinae

Cyclolepidoidei

Conchopomidae

Cyclolepidoti

Congiopodidae

Cyclopteridae

Congridae

Cyematidae

Congrogadidae

Cynodontidae

Copodontidae

Cynoglossidae

.78

Coracidae

Cyprinidae

.65

Cornaspidae

Cornuboniscida

Cyprinodontidae

.79

Cyttidae

.72

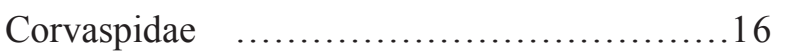

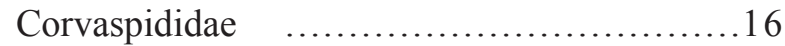

Coryphaenidae .............................77

Cosmolepididae ...............................47

Cosmoptygiidae ..........................47

Cottidae ..................................86

Cranoglanididae $\ldots . . \ldots \ldots \ldots \ldots \ldots . \ldots . \ldots 67$

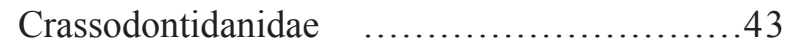

Crassonotidae ...............................43

Cratoselachiidae ............................26

Creediidae .................................84

Crenuchidae ...............................66

Cretatriacanthidae ...........................83

Cretazeidae .................................. 72

Cretoxyrhinidae .............................42

Crossognathidae ...............................59

Crurirajidae ..............................45

Cryphiolepidae ............................47

Cryphiolepididae ..........................47

Cryptacanthodidae ......................86

Cryptobalistinae .............................83

Ctenacanthidae .............................36

Ctenacanthini ................................. 36

Ctenaspidae (.................................18

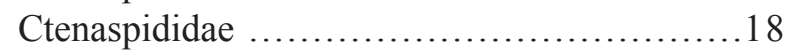

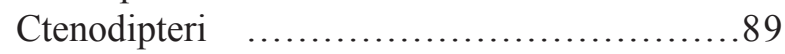

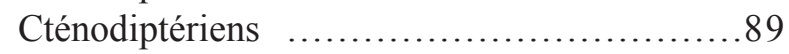

Ctenododipteridae .............................89

Ctenodontidae $\quad$.................................89

Ctenolepides ...............................59

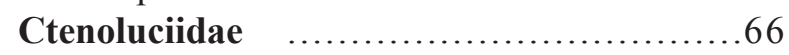

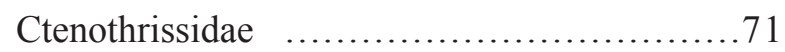

Culmacanthidae .............................33

Cuneatini $\quad$................................55

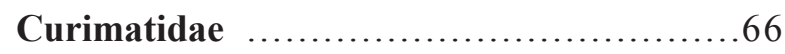

Cyathaspidae ...............................18

\section{D}

Dactylopteridae ...........................75

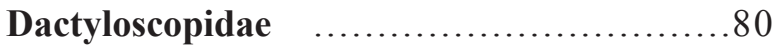

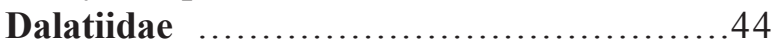

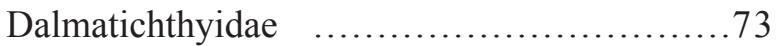

Danionidae $\quad$................................65

Dapedidae ...................................55

Dapediden ....................................55

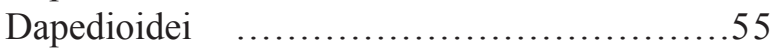

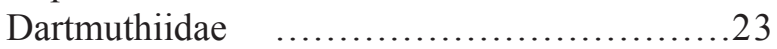

Dasyatidae ................................45

Datnioididae .............................82

Dayongaspidae .............................24

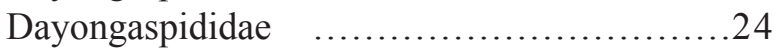

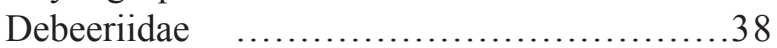

Deltodontidae .................................. 39

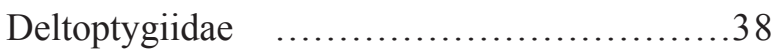

Dendrodonten ............................. 88

Dendrodontidae ............................ 88

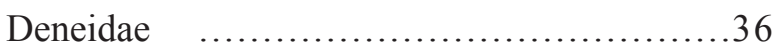

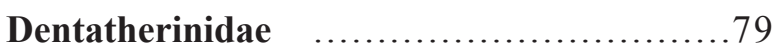

Denticipitidae $\quad . . \ldots \ldots \ldots \ldots \ldots \ldots \ldots . \ldots . \ldots 64$

Dercetidae ...............................69

Dercetiformes ...............................69

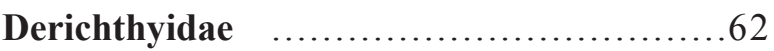

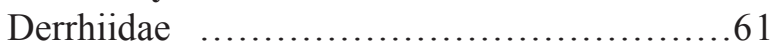

Desmiodontidae ................................37

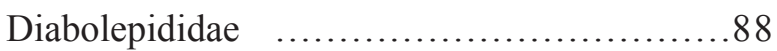

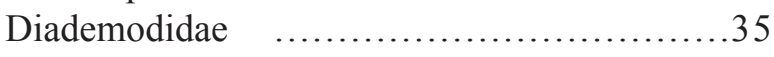

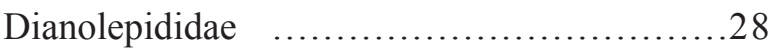

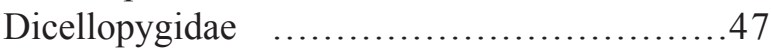

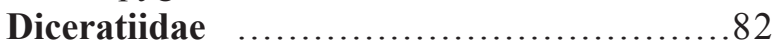

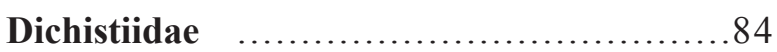

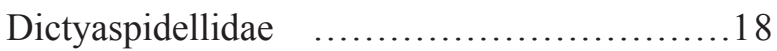

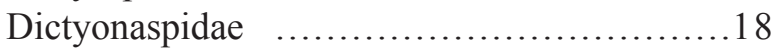

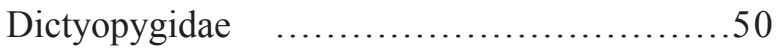

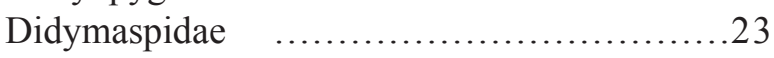




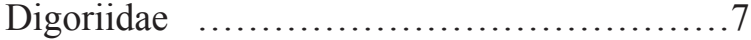

Dinaspidae ................................. 1

Dinaspidellidae $\ldots . \ldots \ldots \ldots \ldots \ldots \ldots \ldots \ldots \ldots \ldots \ldots$

Dinematichthyidae $\quad . . \ldots \ldots \ldots \ldots \ldots \ldots \ldots . \ldots . \ldots . \ldots$

Dinichthidae ................................. 3

Dinichthyidae ...............................32

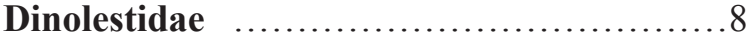

Dinopercidae .............................

Dinopterygidae ..............................73

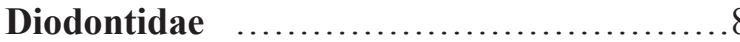

Diodontiides ............................... 83

Diphyodontidae ..............................5

Diplacanthidae ...............................33

Diplaspidae .................................18

Diplocercididae ...............................87

Diplocercidae .................................87

Diplodoselachida................................36

Diplomystidae ..............................64

Diplomystidae .............................66

Diplopteroidei $\quad$................................99

Diplurinae ....................................87

Dipnorhynchidae $\quad$............................88

Dipterichthyidae ...............................74

Dipteridae .....................................89

Diptériens ....................................89

Diretmidae ................................73

Discordichthyidae .............................51

Distichodontidae ............................66

Distobatidae .................................40

Doradidae ...................................67

Dorsetichthyidae ...........................59

Doryaspididae ..............................18

Doryopteri ....................................49

Doryopteridae $\quad$...............................49

Draconettidae ............................... 75

Drepanaspidae ..............................19

Drepanaspididae ...............................19

Drepaneidae ................................. 81

Drepanolepididae ............................21

Ductoridae .....................................77

Dunkleosteidae ...............................32

Duyunoaspidae ..............................25

Duyunolepidae ...............................25

Duyunolepididae ............................25

Dwykiidae .................................51

E

Echeneidae

Echinochimaeridae

Echinorhinidae
Ectosteorhachidae $\quad$...........................91

Edaphaspididae .............................17

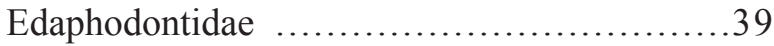

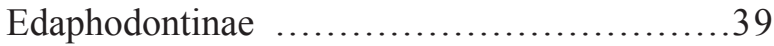

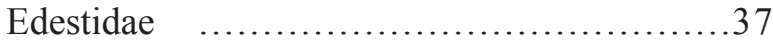

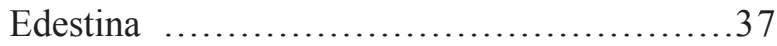

Eekaulostomidae ...........................75

Eestilepididae ................................21

Eglonaspididae ..............................17

Eigiliidae .................................47

Elassomatidae .............................. 84

Elegestolepididae ............................34

Eleginopsidae ...............................85

Eleotridae ................................. 76

Ellimmichthyidae ...........................64

Ellimminae ...................................64

Ellopostomatidae ..........................66

Elonichthyidae ...............................48

Elopidae .................................61

Elpistostegidae ...............................991

Embiotocidae .............................. 78

Emmelichthyidae ......................... 80

Encheliidae ..................................61

Enchodontidae ..............................70

Endeiolepidae ................................19

Endeiolepididae .............................19

Engraulidae ................................64

Enoplosidae ................................84

Eoapogonini .................................76

Eobuglossidae ..............................78

Eocoelopomini …..........................75

Eocottidae ................................... 85

Eodiodontidae ..............................84

Eogastrophinae ..............................76

Eoleiognathinae ...............................81

Eomyctophinae ..............................71

Eonotidanidae (..............................43

Eoplectidae ....................................83

Eoplectinae ................................... 83

Eoplethodidae (.............................60

Eopodoidea ....................................991

Eoptolamnidae ..............................43

Eoscymnidae .................................43

Eosemionotidae ….........................54

Eospinidae .................................... 83

Eostriatolamiidae ............................42

Eotrigonodontidae ............................8 80

Ephippidae ................................ 81

Epigonidae …............................ 84

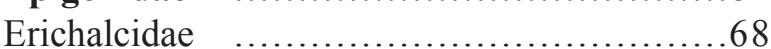


VAN DER LAAN R., Family-group names of fossil fishes

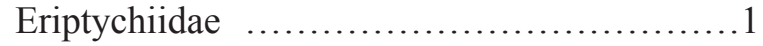

Erisichtheidae .............................58

Erismatopteridae $\quad$............................72

Errolichthyidae .............................52

Erromenosteidae .............................32

Erythrinidae ..............................66

Erythrinolepidae ............................65

Eschmeyeridae ............................. 85

Escuminaspididae ...........................23

Esocidae $\quad$..................................68

Etmopteridae .............................43

Eucitharidae ................................. 78

Euclichthyidae .............................72

Eugaleaspidae (..............................24

Eugeneodontidae .............................37

Eugnathidae ...............................57

Euleptaspididae ...............................30

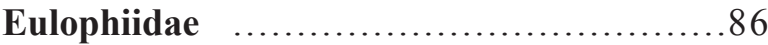

Euphaneropidae ..............................19

Euphyllodontinae $\quad$...........................61

Euporosteidae ................................87

Eurokidae ....................................61

Eurycormidae ................................58

Eurylepidae $\quad$................................51

Eurynotoididae .............................48

Eurynotoidiidae ...............................48

Eurypharyngidae ...........................63

Eurypholidae .................................70

Eurysomidae .................................49

Eustenopteridae .............................992

Euthacanthidae ...............................34

Euzaphlegidae $\quad$...............................74

Evenkiidae ...................................51

Evermannellidae ...........................70

Exelliidae ................................... 80

Exocoetidae ..................................79

F

Falcatidae .36

Ferrifronsidae ...............................68

Fistulariidae …........................... 75

Fistularioididae ..............................75

Fleurantidae $\quad$................................ 89

Fleurantiidae ................................. 89

Fluviphylacidae $\quad$............................ 80

Foreyichthyidae ..............................63

Forficidae ................................79

Fundulidae ...............................79

Furcacaudidae $\quad$...............................20

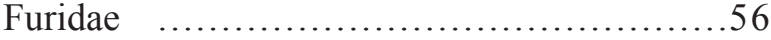

Fuyuanperleididae ..........................54

\section{G}

Gabanellidae ..................................54

Gabanelliidae ................................54

Gabreyaspididae ............................ 17

Gadidae .................................. 73

Gaidropsaridae ............................ 73

Galaxiidae ..................................68

Galeaspidae ..............................24

Galeocerdidae ...........................43

Galkiniidae ....................................58

Ganolytidae ................................64

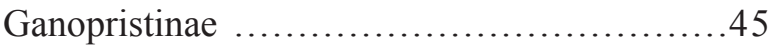

Ganopristodidae ............................. 90

Gantarostrataspidae ........................25

Gantarostrataspididae ........................25

Garganoclupeidae ...........................64

Gasteropelecidae ..........................66

Gasterosteidae ..............................86

Gastromyzontidae .........................66

Gavinaspididae .............................29

Gebrayelichthyidae (........................53

Gempylidae ............................... 74

Gemuendenaspidae (.........................31

Gemuendinidae .............................27

Georgidentidae .............................61

Geotriidae ................................... 15

Geraspididae ...............................25

Gerdalepinae ................................28

Gerpegezhidae ............................75

Gerreidae ................................. 81

Gibberichthyidae ...........................73

Gibbodontidae ...............................53

Gigantactinidae $\quad$........................... 82

Gigantaspididae .............................19

Gigantodontidae .............................56

Giganturidae ............................. 70

Gillicinae .................................60

Gilpichthyidae $\quad$.............................15

Ginglymostomatidae (......................41

Girellidae ................................ 84

Gladiopycnodontidae ........................53

Gladiobranchidae ............................33

Glaucosomatidae ........................... 84

Glaucostegidae ….........................45

Glyptolaemini …........................ 90

Glyptolépides ............................... 88

Glyptopomidae .............................91 
Gnathanacanthidae

Gnathorhizidae

Gobiesocidae

Gobiidae

Gobionidae

Gonatodidae

Gonorynchidae

Gonostomatidae

Goodeidae

Goodradigbeeonidae

Grammatidae

Grammicolepididae

Greenwoodellidae

Gregoriidae

Grenfellaspididae

Groenlandaspididae

Gronlandaspididae

Grossaspidae

Guerichosteidae

Guildayichthyidae

Gurgesiellidae

Gymnarchidae

Gymnoniscidae

Gymnotidae

Gymnuridae

Gyracanthidae

Gyracanthididae

Gyrinocheilidae

Gyrodontidae

Gyrolepidotidae

Gyroptychiidae

Gyrosteini

\section{H}

Habroichthyidae

Hadrodontidae

Hadronectoridae

Hadrosteidae

Haemulidae

Hagiangellidae

Haimirichiidae

Halecidae

Halécoides

Halecopsidae

Halosauridae

Hanyangaspidae

Hanyangaspididae

Hapalogenyidae

Haplolepidae

Hardistiellidae

\section{.85}

.90

.80

.76

.65

. .49

..65

..68

. .79

.31

. .78

.72

60

.37

.27

.30

.30

.28

.19

.50

.45

.63

. .48

.66

..45

..34

.34

.65

.53

.48

.91

.52

$$
\text { H }
$$

Harpacanthidae ...............................37

Harpagiferidae ............................ 85

Heintzaspidae ...............................29

Helenolepididae .............................20

Helicoprionidae ...............................37

Helodontidae ................................... 38

Helodontinae .................................. 38

Helostomatidae .............................76

Hemerocoetidae ...........................8 84

Hemicyclaspinae ..........................22

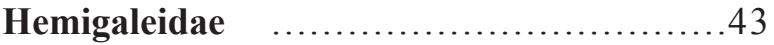

Hemingwayidae ...............................77

Hemiodontidae ...............................66

Hemiramphidae ...........................79

Hemiscylliidae ..............................4 41

Hepsetidae .................................66

Heptapteridae ...........................67

Heptranchidae ..............................43

Heslerodidae ...................................36

Heterenchelyidae ...........................62

Heterocercoidei ..............................49

Heterodontidae ............................4 41

Heterogaspidae ...............................30

Heteropneustidae ..........................67

Heterosteiden ...................................31

Heterostii ................................... 31

Heterosteidae ....................................31

Hexagrammidae ............................86

Hexanchidae ................................43

Hexatrygonidae ............................45

Hibernaspididae ..............................17

Himantolophidae ............................82

Hiodontidae ................................63

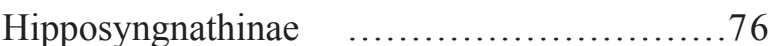

Hirellidae ...................................22

Hispidoberycidae ...........................73

Histiothrissinae ...............................46

Hoelaspididae ................................24

Holocentridae ...............................74

Holodipterida ................................89

holodipterids ..................................89

Holodontidae ................................89

Holohalaeluridae ...........................43

Holonematidae ................................30

Holonemidae ..................................30

Holoptychii $\quad$.................................. 88

Holoptychiidae $\ldots \ldots \ldots \ldots \ldots \ldots \ldots \ldots \ldots \ldots$

Holosteinae .................................70

Holuridae ..................................48

Homalodontidae .............................40 
VAN DER LAAN R., Family-group names of fossil fishes

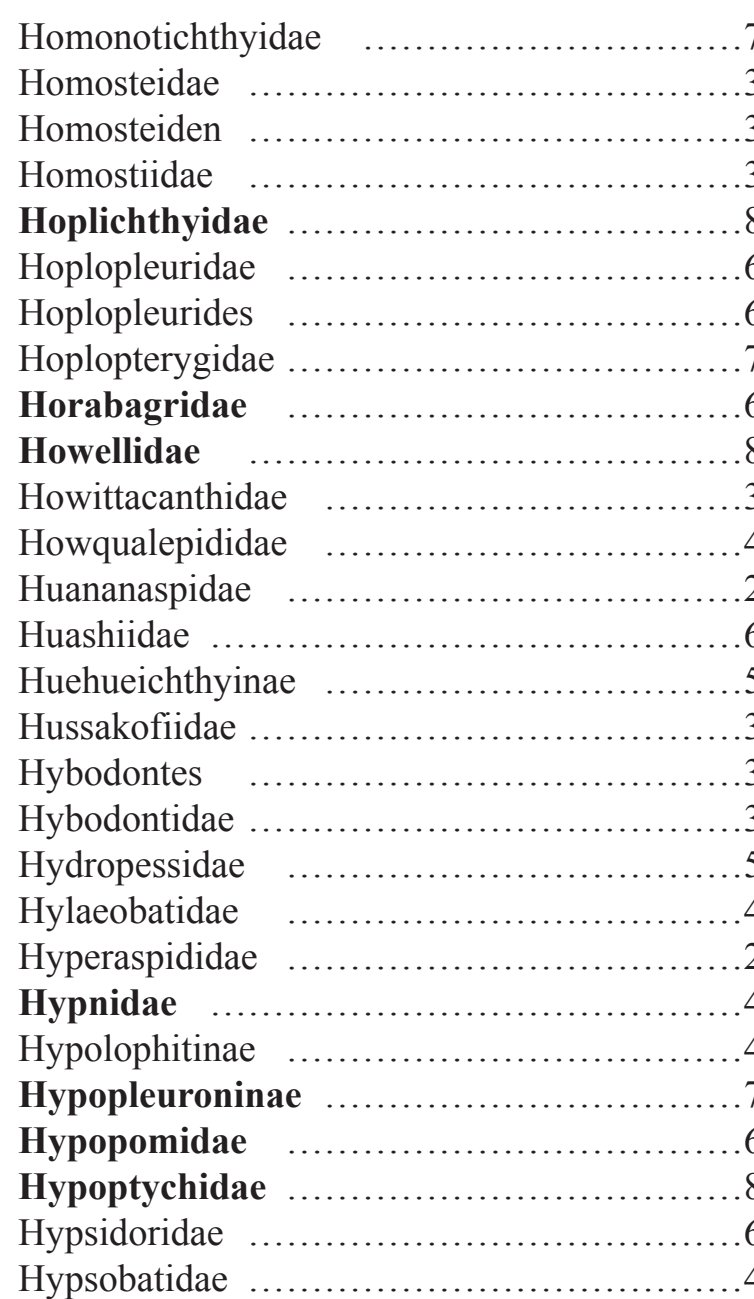

\section{I}

Ichthyodectidae ..............................6 60

Ichthyokentemidae ..........................59

Ichthyosauroididae .............................. 32

Ichthyotringidae .............................69

Icosteidae ................................. 74

Ictaluridae ..................................67

Ictinocephalidae .............................33

Igornichthyidae ..............................50

Iguanodectidae .............................66

Incisoscutidae ................................. 31

Indostomidae $\quad$..............................76

Inichthyidae f...............................51

Iniopterygidae $\quad$................................37

Ionoscopidae ..............................57

Ioscionidae ................................. 82

Ipnopidae ................................. 70

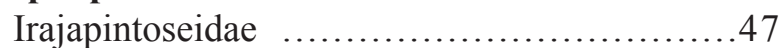

Irregularaspidae .............................. 18

Ischnacanthidae ..............................33

Ischyrhizidae .................................45
Isonidae ................................... 79

Isopholidae .................................5 57

Isozenidae ................................. 72

Istiophoridae ............................... 77

$\mathbf{J}$

Jaekelaspidae ..................................30

Jagorinidae ..................................27

Jalodontidae ...................................

Jamoytiidae ................................19

Janassidae ..................................... 38

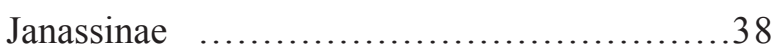

Jekelotodontidae (...........................42

Jianghanichthyidae ...........................65

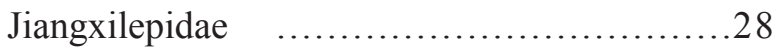

Jiaohichthyidae ...........................663

Jiuquanichthyidae .........................66

Johnlonginae ..............................42

Joleaudichthyidae ..........................78

Jordaniidae ................................ 86

Judeichthyidae $\quad$............................65

\section{K}

Kalanaspididae ...............................22

Kannathalepididae .............................34

Karaunguriidae ...............................46

Kathemacanthidae ...........................34

Katoporidae (...............................22

Katoporodidae (..............................22

Kenderlichthyidae ...........................51

Kendrickichthyidae $\quad$........................... 31

Kentuckiidae ................................48

Kenyaichthyidae (............................79

Kiaeraspididae .............................23

Kiaeraspinae ...............................23

Kipalaichthyidae (..........................63

Kneriidae ..................................65

Kolymaspidae ............................26

Kolymaspididae ............................26

Koonwarriidae ...............................59

Kryptoglanidae .............................67

Kuhliidae .................................... 84

Kujdanowiaspidae ...........................29

Kujdanowiaspididae ........................29

Kurtidae …..............................76

Kushlukiidae .................................81

Kuyangichthyidae ...........................63

Kyphosichthyidae ............................55

Kyphosidae ................................. 84 


\section{L}

Labridae

Labrisomidae

Lacantuniidae

Lactariidae

Laeliichthyinae

Lagarodontidae

Lamiostomatidae

Lamnidae

Lampridae

Lamprotolepididae .............................99

Lanarkiidae ...................................20

Larnovaspididae ...............................18

Lasaniidae ..................................19

Lateolabracidae $\quad$...........................84

Latimeriidae .............................. 87

Latridae ................................. 84

Laugiidae ................................... 87

Lawniidae ...................................48

Lebachacanthidae ............................36

Lebiasinidae ..............................66

Leiodontidae ..................................37

Leiognathidae ..............................81

Leiosteidae ..................................... 32

Leiosteiden ....................................32

Lenacanthidae ..............................33

Lepadolepididae ..............................27

Lepadolepinae (..............................28

Lepidaspididae $\quad$...............................16

Lepidogalaxiidae ..........................68

Lépidoïdes ..................................55

Lepidosirenidae $\quad$............................90

Lepidostei ....................................55

Lepisosteidae ...............................55

Leptobarbidae .............................65

Leptobramidae ..............................77

Leptochariidae ..............................43

Leptolepidae ..................................59

Leptolépides .................................59

Leptolepididae...............................59

Leptoscopidae ............................84

Leptosteidae $\quad$..................................32

Lestidiidae ................................. 70

Lethrinidae $\quad$................................82

Leuciscidae ...............................65

Libanechelyidae .............................662

Libotoniidae ................................ 72

Ligulellidae ...............................58

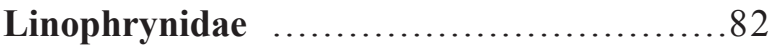

Liodesmidae

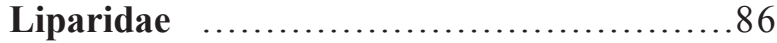

Lissoberycinae $\quad$...............................73

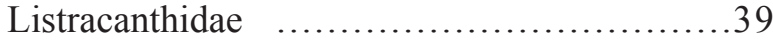

Liuaspidinidae $\quad$..............................24

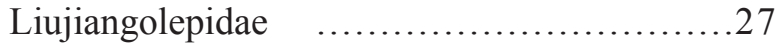

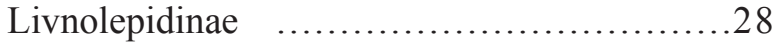

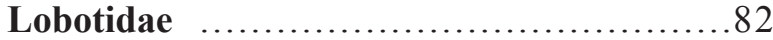

Loganellidae ...............................21

Loganelliidae ............................21, 22

Loganelliinae ................................22

Loganiidae ..................................21

Lombardinidae ..............................52

Lonchidiidae ...............................40

Londichiidae ................................40

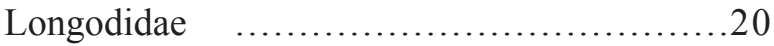

Lophichthyidae ............................. 82

Lophiidae ................................. 82

Lophiostomatoidei ............................57

Lophosteidae ................................46

Lophotidae ............................... 71

Loricariidae ..............................67

Lotidae ................................... 73

Lugalepididae ................................40

Luganoiidae .................................55

Luisiellidae ..................................60

Lumpenidae ................................86

Lunaspidae ..................................29

Lungmenshanaspididae ......................25

Lutjanidae ................................. 82

Luvaridae .................................. 81

Lycoclupeidae .............................64

Lycopteridae ................................63

Lyktaspididae ................................19

\section{M}

Machaeracanthidae $\quad$............................32

Macromesodontidae ..........................53

Macropetalichthidae ........................29

Macropetalichthyidae ........................29

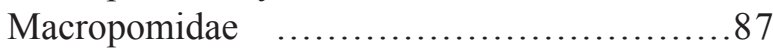

Macrosemii …...........................56

Macrosemiidae ..............................56

Macrothyraspididae ........................25

Macrouridae ................................. 72

Macruronidae ...............................72

Majokiidae ...................................59

Malacanthidae …........................... 80

Malapteruridae ..........................67

Manchurichthynae ...........................63

Mandageriinae ..............................992 
VAN DER LAAN R., Family-group names of fossil fishes

Masillosteinae

Massalongiidae

Mastacembelidae

Matzichthyinae

Mawsoniidae

Mayomyzontidae

Mcconichthyidae

Mcmurdodontidae

Mediaspidae

Medoevididae

Megachasmidae

Megalichthyidae

Megalopidae

Megaluridae

Megascyliorhinidae

Megistolepidinae

Melamphaidae

Melanocetidae

Melanonidae

Melanotaeniidae

Menaspidae

Menidae

Merlucciidae

Mesacanthidae

Mesogasteridae

Mesolepidae

Mesturidae

Metaveliferinae

Microbrachiidae

Microbrachiinae

Microcanthidae

Microhoplonaspididae

Microlepidoti

Microstomatidae ........................6 68

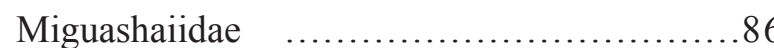

Milananguillidae ...........................662

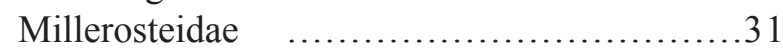

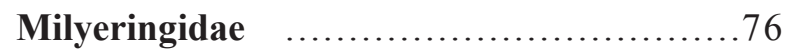

Mimetaspididae .............................24

Mimiidae $\quad$................................48

Minicranidae ................................27

Mitsukurinidae ...........................44 42

Mjollnirulidae $\quad$..............................58

Mobulidae .................................46

Mochokidae ...............................67

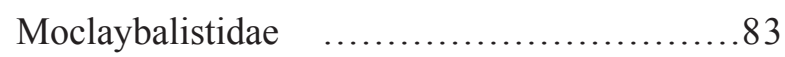

Molidae .................................83

Monacanthidae ..........................8 83

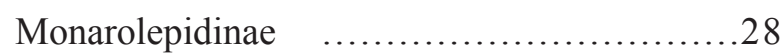

Monaspidae

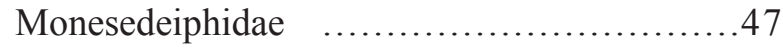

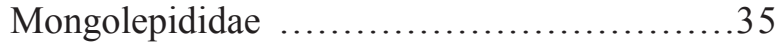

Monocentridae .............................73

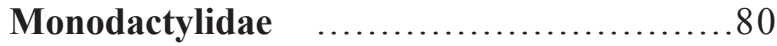

Monognathidae .........................6 63

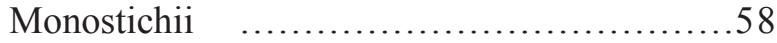

Mordaciidae ..............................15

Moridae ..................................72

Moringuidae .............................62

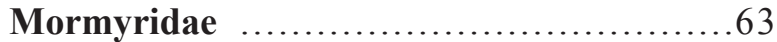

Moronidae ..................................80

Moythomasiidae .............................48

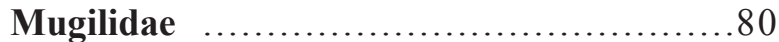

Mullidae $\quad$.................................. 75

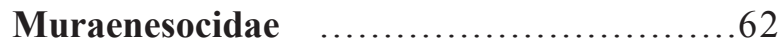

Muraenidae ...............................62

Muraenolepididae $\quad$........................72

Myctophidae ..............................71

Myliobatidae ..............................45

Myllokunmingiidae $\quad . . . . . . . . . . . . . . . . . . . .15$

Mylomyridae .............................61

Mylostomatidae ..............................31

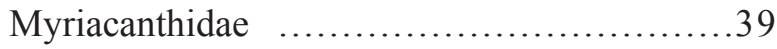

Myrocongridae ..........................62

Myxinidae ...............................15

\section{$\mathbf{N}$}

Nahuaichthyinae $\quad$.........................53

Nandidae ..................................77

Nanpanaspidae (............................25

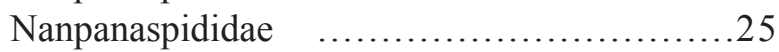

Narcinidae .................................44

Nardoechelyidae $\quad$..........................62

Nardoichthyidae ............................76

Nardorexidae ................................69

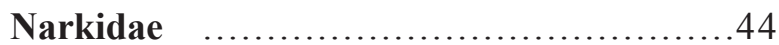

Natlaspididae ...............................16

Nectaspididae ................................24

Neeyambaspididae ...........................26

Nemacheilidae .............................66

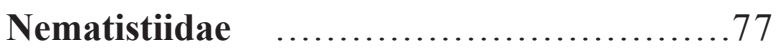

Nematogenyidae ..........................67

Nematoptychiidae ..........................51

Nemichthyidae $\ldots . \ldots \ldots \ldots \ldots \ldots \ldots \ldots \ldots . \ldots 62$

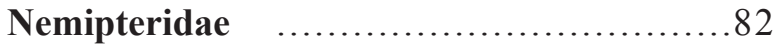

Neocassandridae $\quad$.............................71

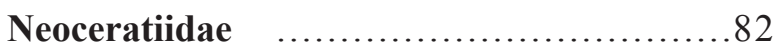

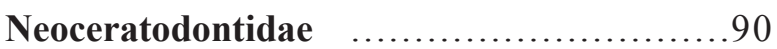

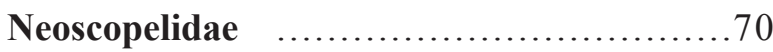

Neosebastidae $\quad$............................85 
Neozoarcidae

Nessariostomidae

Nettastomatidae

Nikoliviidae

Niobraridae

Niphonidae

Nomeidae

Normanichthyidae

Notacanthidae

Notelopidae

Nothobranchiidae

Notogoneidae

Notopteridae

Notorhizodontidae

Notosudidae

Nototheniidae

Nunavutiidae

Nursalliidae

Nybelineidae

O

Obaichthyidae

Obrucheviidae

Obruchevodidae

Occithrissopidae

Odontaspididae

Odontobutidae

Odontotodontidae

Oeselaspidae

Oeseliidae

Ogcocephalidae

Olbiaspididae

Oligopleuridae

Omalodontidae

Omosomopsidae

Onchidae

Oneirodidae

Oniscolepididae

Onychodontidae

Ophichthidae

Ophidiidae

Ophiopsidae

Ophiopsiellidae

Opisthocentridae

Opisthomyzonidae

Opisthoproctidae

Opistognathidae

Oplegnathidae

Orectolobidae

Oreosomatidae
.86

.26

62

.20

61

.85

.75

..85

61

. .60

.79

.65

.63

. .92

.70

.85

5

.19

.38

.60

42

.76

.23

.23

.20

.82

.17

.59

..35

.73

32

.82

$\ldots 16$

.88

.63

.74

.57

.57

.86

.77

.68

.78

.84

. .41

.72
Orestiidae ….............................79

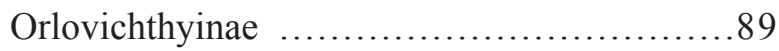

Orodontidae ..........................................

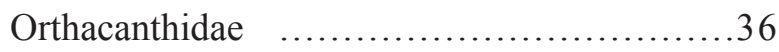

Orthacodidae .................................41

Orthechinorhinidae $\quad$........................44

Orthogonikleithridae $\ldots \ldots \ldots \ldots \ldots \ldots \ldots \ldots . \ldots 68$

Oshuniidae .................................57

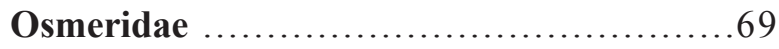

Osmeroididae ................................61

Osorioichthyidae ...........................49

Osphronemidae ...........................76

Ospiidae .....................................56

Ostariostomidae ..............................63

Ostenoselachidae ............................40

Osteoglossidae ..............................63

Osteolepididae (...............................91

Ostraciidae ................................83

Ostracoberycidae .......................... 84

Otodontidae ................................41

Oxudercidae ................................. 76

Oxygnathidae .................................49

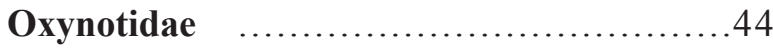

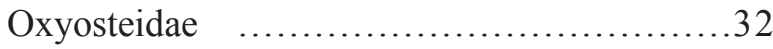

\section{$\mathbf{P}$}

Pachycormidae ............................ 57, 58

Pachyosteidae ................................. 32

Pachyrhizodontidae .........................60

Paedocyprididae ...........................65

Palaeacanthaspidae (........................26

Palaeaspidae ..............................18

Palaeobalistidae ..............................53

Palaeobergiidae ...............................51

Palaeocarcharidae ............................41

Palaeocentrotidae .............................71

Palaeodontidae ..............................15

Palaeoesocidae .............................68

Palaeolabridae ...............................57

Palaeolycinae ................................... 70

Palaeoniscidae ..............................49

Palaeoniscini ................................49

Palaeorhynchidae ..........................77

Palaeoscylliidae .............................43

Palaeospinacidae $\quad$............................41

Palaeospinacinae $\quad$.............................41

Palaeospondylidae ............................34

Paleopsephurinae ..........................52

Pambulaspidae .............................28

Pambulaspididae (...........................28 
VAN DER LAAN R., Family-group names of fossil fishes

Panderichthyidae $\quad$.............................. 9

Pangasiidae .................................6

Pantanodontidae ............................79

Pantodontidae ..............................63

Panxianichthyidae ............................56

Panxiosteidae ................................... 31

Paphosiscidae ................................51

Parabatrachidae .............................91

Parabuchanosteidae ..........................31

Paracentrophoridae .........................56

Paracestrationidae ............................41

Paraclupeidae .................................64

Paracoracidae .................................42

Paraeoliscidae (...............................75

Paraisuridae ................................43

Paralbulinae ................................61

Paralepididae $\quad$.............................. 70

Paralepidotidae ..............................5

Paralichthodidae ............................. 7

Paralichthyidae ............................ 78

Paramblypteridae ............................49

Parameteoraspididae .........................23

Paranguillidae (..............................62

Paraorthacodidae ............................41

Parapalaeobatidae ............................45

Paraplesiobatidae ............................26

Parapodoidea ..................................991

Parascorpididae ............................. 80

Parascylliidae ...............................41

Parasemionotidae .............................56

Parasynarcualidae ..............................75

Paratrachinotini ...............................77

Paraulopidae ................................ 70

Parazenidae .................................72

Parechelidae ...............................66

Parexidae ..................................... 34

Parodontidae (.............................66

Pataecidae .................................. 85

Patavichthidae .............................62

Patavichthyidae ............................62

Pateropercidae ...............................71

Pattenaspididae .............................24

Pattersonellidae ..............................68

Pattersonelloidea ...........................68

Pattersonichthyidae ..........................71

Pegasidae ....................................75

Peipiaosteidae $\quad$................................52

Pelecopteridae ................................58

Pelecorapidae ..................................5

Peltopleuridae
Pempheridae .............................. 84

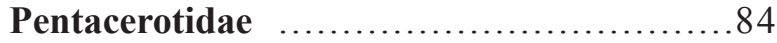

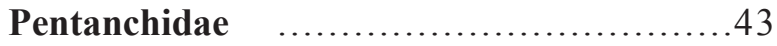

Pentathyraspididae ...........................25

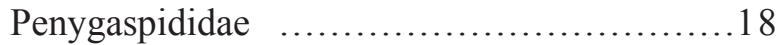

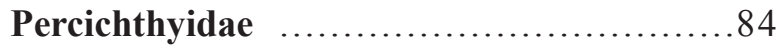

Percidae ................................... 85

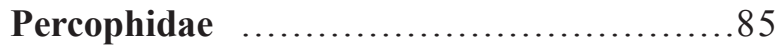

Percopsidae ................................72

Peripristidae ................................... 38

Peristediidae ................................. 86

Perleididae ..................................54

Perryenidae ................................ 85

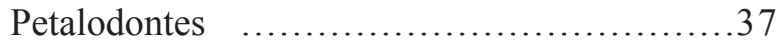

Petalodontidae .................................37

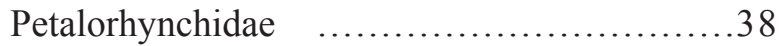

Petromyzontidae ...........................15

Pezopallichthyidae ...........................20

Phallostethidae ................................79

Phaneropleuridae ..........................89

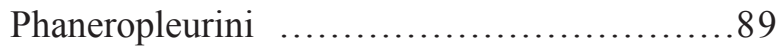

Phanerorhynchidae ..........................50

Phareodontidae .............................63

Pharmacichthyidae ...........................71

Pharyngodopilidae .............................81

Pharyngolepidae ..............................20

Pharyngolepididae ............................20

Phialaspididae (...............................17

Phlebolepidae ..................................222

Phlebolepididae ...............................22

Phlyctaenaspi ................................. 30

Phlyctaenaspiden $\quad$...............................30

Phlyctaenaspinae $\quad$............................... 30

Phlyctaeniidae …..........................30

Phoebodontidae ..............................35

Pholidae ................................... 86

Pholidichthyidae ...........................78

Pholidophori .................................58

Pholidophoridae ...............................58

Pholidopleuridae (...........................54

Pholidosteidae (..............................29

Pholidosteiden ..............................29

Phorcynidae ..................................41

Phosichthyidae .............................68

Phreatobiidae ..............................67

Phycidae …................................73

Phyllodidae $\quad$................................61

Phyllodontidae ..............................61

Phyllolepidae ................................29

Phyllolepididae ….........................29 


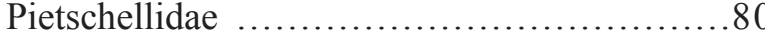

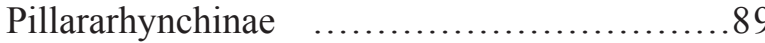

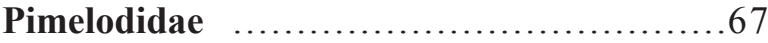

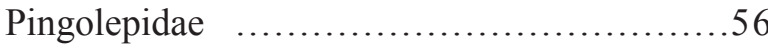

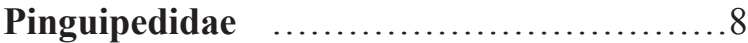

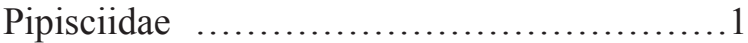

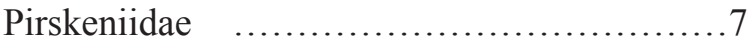

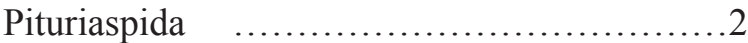

Pituriaspididae ........................... 25, 2

Placosteinae (................................19

Platycephalichthyinae $\quad$......................99

Platycephalidae .............................8

Platyrhinidae $\quad$...............................45

Platysellidae ................................52

Platysiagidae .................................54

Platysomatoidei …...........................49

Platysomidae ..................................49

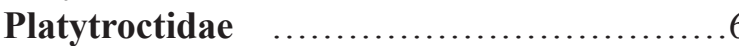

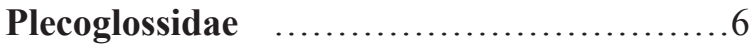

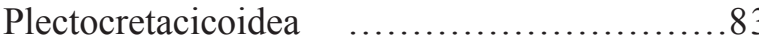

Plectrogeniidae ............................. 85

Pleiopteri ….............................90

Plesiobatidae .................................45

Plesiopidae ............................... 78

Plethodidae ....................................60

Pleuracanthidae ...............................36

Pleurolepididae ...............................55

Pleuronectidae .............................. 78

Pleuropholidae ...............................58

Plotosidae ................................67

Plourdosteidae $\quad$................................. 31

Podolaspinae .................................18

Poeciliidae .................................. 80

Poecilopsettidae $\quad$............................. 78

Polyacrodontidae ..............................40

Polyaspidae .................................29

Polybranchiaspidae ............................25

Polybranchiaspididae .........................25

Polycentridae ..............................78

Polymerichthyidae .............................70

Polymerolepididae ..............................35

Polymixiidae ..................................73

Polynemidae .................................. 77

Polyodontidae .............................5

Polyprionidae $\quad$.............................. 84

Polypteridae .................................51

Polyrhizodontinae ..............................37

Polysentoridae $\quad$.................................34

Polzbergiidae ..................................54

Pomacanthidae .

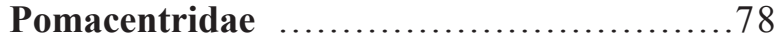

Pomatomidae ............................ 75

Poracanthodidae ................................ 33

Poraspidae ..................................17

Porolepidae ................................ 88

Porolepididae ............................... 88

Porophoraspidae ............................. 16

Potamotrygonidae .........................45

Powichthyidae .............................. 88

Powichthyiidae .............................. 88

Praesemionotidae .............................56

Premontreinae $\quad$..............................43

Prescottaspidae ..............................29

Priacanthidae (............................. 82

Prionolepididae ................................ 70

Priscacaridae ................................. 78

Priscosturioninae $\quad$............................52

Pristidae $\quad$................................45

Pristigasteridae ..............................64

Pristiophoridae ...............................44

Pristodontidae ........................... 37, 38

Pristolepididae ...............................77

Procatopodidae .............................. 80

Procephalaspididae $\quad$.........................23

Prochilodontidae ............................66

Procondylolepidae ...........................27

Profundulidae ..............................79

Progymnodontinae ............................. 84

Prohaleciteidae ................................58

Promecosominidae ............................56

Pronotacanthidae (............................61

Propenserinae ................................. 15

Propercarinidae ...............................75

Propteroidei ................................56

Proscinetinae .................................55

Proscylliidae .................................43

Protacanthodinae (.............................83

Protacrodontidae .............................39

Protacrodontoidea .............................39

Protanguillidae ...............................62

Protaspidae ..................................... 18

Protelopidae ...............................58

Proteomyridae ...............................62

Protobalistidae $\quad$...............................83

Protobramidae ................................60

Protoceratodontidae .......................... 90

Protodontidae ................................... 34

Protopsephurinae $\quad$.............................52

Protopteraspididae .............................19

Protopteridae $\quad$............................... 90 
VAN DER LAAN R., Family-group names of fossil fishes

Protosphyraenidae

Protospinacidae

Protostomiatidae

Protosyngnathidae

Protozeidae

Protriacanthidae

Psammodonten

Psammodontidae

Psammolepididae

Psammorhynchinae

Psammosteidae

Psephaspididae

Psephodontidae

Psephodontinae

Psettodidae

Pseudaphritidae

Pseudobeaconiidae

Pseudoberycidae

Pseudocarchariidae

Pseudochromidae

Pseudocoracidae

Pseudodalatiidae

Pseudodontichthyidae

Pseudoechinorhinidae

Pseudoginglymostomatidae

Pseudomonocentrididae

Pseudomugilidae

Pseudonotidanidae

Pseudopetalichthyidae

Pseudophyllodontinae

Pseudopimelodidae

Pseudorhinidae

Pseudoscapanorhynchidae

Pseudoscaphirhynchinae

Pseudotriakidae

Pseudotrichonotidae ....................... 70

Pshekhadiodontinae .......................... 84

Pshekhagnathinae ..............................76

Psilorhynchidae ..........................65

Psychrolutidae ................................86

Pteraspididae ................................ 18

Pterichthidae ..................................28

Pterichthyodidae f............................28

Pterolepidae ...................................20

Pterolepidopidae (............................20

Pteroscylliinae .............................43

Pterygocephalidae ...........................75

Pterygolepididae $\quad$............................19

Ptilichthyidae f.............................86

Ptychoceratodontidae ..........................90
Ptychocoracidae ..............................42

Ptychodontidae ...............................40

Ptychodontinae $\quad$...............................40

Ptycholepidae .................................5 51

Ptycholepididae ..............................51

Ptychotrygonidae ............................45

Ptyctodontidae .............................29

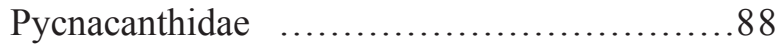

Pycnodontae ................................53

Pycnodontes ...............................5 53

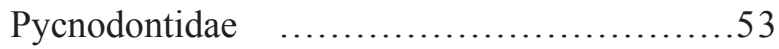

Pycnosteidae .................................19

Pycnosteroididae ............................71

Pygaeidae ..................................77

Pygopteri ...................................48

Pygopteridae .................................48

\section{Q}

Qingmenaspididae ...........................25

Quaesitoberycidae ..........................73

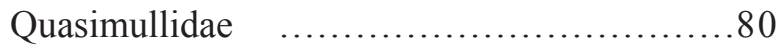

Quasipetalichthyidae .......................29

Qujinolepidae ...............................27

$\mathbf{R}$

Rachycentridae ..............................77

Radiicephalidae ........................... 71

Radotinidae ................................26

Rajidae ..................................45

Ramsaasalepididae ...........................20

Ranicipitidae ............................... 72

Raphiosauridae ............................60

Rebellatricidae ............................... 87

Redfieldiidae ................................50

Regalecidae ...............................71

Remigolepidae ............................27

Remigolepididae (............................27

Repropcidae .................................81

Retropinnidae ............................69

Rhabdodermidae ............................87

Rhabdolepididae...........................48,49

Rhachiosteidae ................................ 31

Rhadamantidae ................................39

Rhadinichthyidae ...........................49

Rhamphexocoetinae ........................79

Rhamphichthyidae .........................66

Rhamphocottidae ........................... 86

Rhamphognathidae (.......................79

Rhamphosidae .............................75

Rharbichthinae .............................70 
Rhincodontidae

Rhinellidae

Rhinidae

Rhinobatidae

Rhinochimaeridae

Rhinodipteridae

Rhinopteraspiden

Rhinopteraspididae

Rhinopteridae

Rhinorajidae

Rhinosteidae

Rhizochlatridae

Rhizodontidae

Rhizodopsidae

Rhombodipteridae

Rhombodontidae

Rhombosoleidae

Rhyacichthyidae

Rhynchodipteridae

Rhynchodonta

Rhynchodontidae

Rhyncholepidae

Rhyncholepididae

Ritidae

Rivulidae

Robertanniidae

Rogeniidae

Rondeletiidae

Rostraspinae

Rostrati

Rostrorajinae

Rubiesichthyinae

Ruffoichthyinae

S

Saccopharyngidae

Sagenodontidae

Salangidae

Salminopsidae

Salmonidae

Samaridae

Sanchaspidae

Sanchaspididae

Sandiviidae

Sandiviinae

Sanqiaspidae

Sanqiaspididae

Sardinioididae

Sasseniidae

Saurichthyidae
.41 Saurichthyoidei

Saurocephalidae

Saurodipteridae

.60

Saurodontidae

Sauropsidae

Saurorhynchidae

Saurotomini ................................57

Scanilepidae ................................51

Scanilepididae ..............................51

Scapanorhynchidae $\quad$.........................42

Scatophagidae $\ldots \ldots \ldots \ldots \ldots \ldots \ldots \ldots \ldots . \ldots . \ldots 1$

Scaumenaciidae .............................89

Sceletophoridae ..............................48

Schilbeidae ................................67

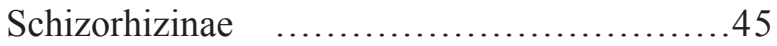

Schizurichthyidae ..........................50

Sciaenidae .................................. 82

Sclerodidae ....................................23

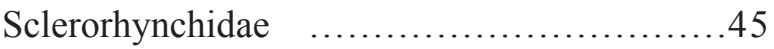

Scolenaspididae .............................23

Scoloplacidae ............................67

Scombridae ...............................75

Scombrolabracidae ........................75

Scombropidae .............................75

Scopelarchidae ............................70

Scophthalmidae ..........................78

Scorpaenichthyidae .........................86

Scorpaenidae ..............................85

Scorpididae .................................84

Scroteidae ...................................49

Scutatuspinosinae ..........................64

Scyliorhinidae .............................4 43

Scytalinidae ............................. 86

Sebastidae .................................... 85

Sedenhorstiidae ..............................61

Selenosteidae ...................................32

Semionotidae .............................55, 56

Semiophoridae ............................. 80

Septentrioniidae ..............................20

Serpenticobitidae ...........................66

Serranidae .................................. 85

Serrasalmidae .............................66

Serratolamnidae ..............................43

Serrilepidae ................................70

Serrivomeridae .............................62

Setarchidae ............................... 85

Shieliidae .................................22

Shiqianolepidae ...............................35

Siberiaspididae .............................17 
VAN DER LAAN R., Family-group names of fossil fishes

Sibyrhynchidae

Siganidae

Signeuxellidae

Sillaginidae

Siluridae

Silurolepidae

Sinacanthidae

Sinamiidae

Singididae

Sinipercidae

Sinogaleaspidae

Sinogaleaspididae

Sinolepidae

Sisoridae

Siyuichthyidae

Skalviaspididae

Soleidae

Solenorhynchinae

Solenosteidae

Solenostomidae

Solnhofenamiinae

Somniosidae

Sorbinicharacidae

Sorbinichthyidae

Sorbininardidae

Sorbinipercidae

Spaniodontidae

Sparidae

Spathobatidae

Speonesydrionidae

Sphaerodini

Sphaerodontidae

Sphaerolepidae

Sphenacanthidae

Sphenocephalidae

Spherosteinae

Sphyraenidae

Sphyrnidae

Spinacanthidae

Squalicoracidae

Squalidae

Squalorajidae

Squatinactidae

Squatinidae

Stegostomatidae

Stegotrachelidae ...

Steinbachodontidae

Steindachneriidae

Stensiöellidae

Stephanoberycidae
.37

81

.52

..81

..67

.27

.33

.57

.63

.84

.24

.24

.27

.67

.58

.18

.78

. .76

.31

. .76

.57

. .44

.58

..64

.65

81

.69

. .82

.44

.88

.52

52,56

.48

.40

.72

.52

.77

.43

83

42

.44

.38

.36

.44

.41

.48

.40

.72

.26

.73
Sternoptychidae

Sternopygidae $\quad$.............................66

Stethacanthidae ...............................36

Stichaeidae (...............................86

Stichocentridae .............................74

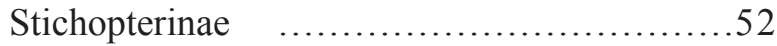

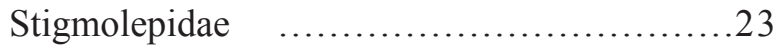

Stomiahykidae $\quad$.............................89

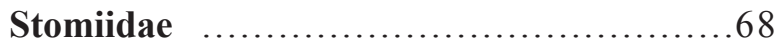

Stratodontidae .............................69

Strepheoschemidae (........................48

Striatolamidae (..............................42

Striatolamiidae ..............................42

Stroinolepididae .............................21

Stromateidae ................................ 75

Stroteidae .................................50

Sturionidae ...............................52

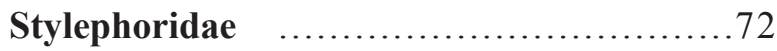

Stylodontes ................................55

Stylodontidae ...............................55

Styracopteridae .............................51

Sudidae ..................................... 70

Sundadanionidae ..........................65

Superciliaspididae ...........................23

Syllaemidae $\quad$.................................59

Symmoriidae .................................36

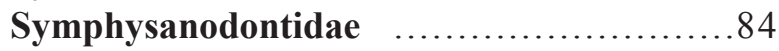

Synanceiidae ............................... 85

Synaphobranchidae .......................62

Synaucheniidae ...............................32

Synbranchidae .............................76

Synechodontidae ...........................41

Syngnathidae $\quad$..............................76

Synodontidae ...............................70

\section{T}

Taemasosteidae .................................31

Tahulalepididae ..............................20

Talivaliidae ...................................21

Tamiobatidae ....................................

Tanichthyidae $\quad$..............................65

Tannuaspididae (...............................23

Tarrasiidae ....................................50

Tarumaniidae .............................66

Tazakidae .....................................34

Tchunacanthidae ...........................33

Tegeolepidae ...............................48

Teleopterinidae .............................51

Telmatherinidae ..........................79

Tenizolepidinae ..............................28 
Tenuicentridae

Tenuicentrinae

Tepexichthyinae

Terapontidae

Tesakoviaspididae

Tesseraspidae

Tesseraspididae

Tetanopsyridae

Tetrabrachiidae

Tetragonuridae

Tetraodontidae

Thaiodontidae.

Thalasseleotrididae

Thaumatichthyidae

Thaumaturidae

Thelodontidae

Thoracopteridae

Thorectichthyinae

Thressopina

Thrinacodontidae

Thrissonotidae

Thrissopatrinae

Thryptodontidae

Thursiidae

Thyestidae

Thysanolepididae

Thysanolepidinae

Tiaraspididae

Tienshaniscidae

Timanaspidinae

Tincidae

Titanichthyidae

Tjalvidae

Tolypaspidae

Tolypelepidae

Tomognathidae

Torosteidae

Torpedaspididae

Torpedinidae.

Tortonesidae

Tortonesiidae

Toxotidae

Trachichthyidae

Trachinidae

Trachipteridae

Trachosteidae

Trachyacanthidae

Trachyrincidae

Trapeziolepidoidei

Traquairaspidae
.74

.74

.53

..84

.16

.16

. .16

.33

.82

.75

.83

39,40

.76

.82

.69

.21

.54

.64

..60

..35

.49

.60

.60

.91

.23

. .91

.91

..30

..46

.23

..65

.32

.58

.17

.17

.69

31

.18

44,66

. .81

.81

.77

. .73

.85

.71

.31

..38

.72

.57

.17
Traquairaspididae ...........................16

Traquairiidae ................................ 38

Trebicianiidae .............................. 71

Tremataspidae $\quad$.............................23

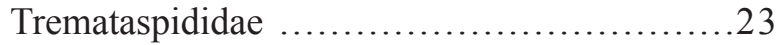

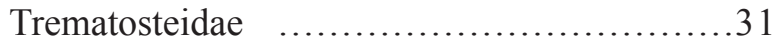

Trewavasiidae $\quad$.............................53

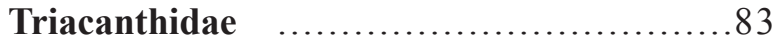

Triacanthodidae $\quad \ldots \ldots \ldots \ldots \ldots \ldots \ldots \ldots \ldots . \ldots \ldots$

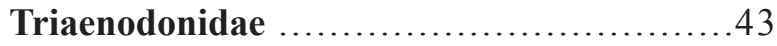

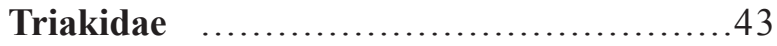

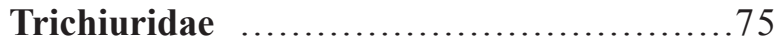

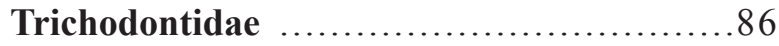

Trichomycteridae .........................67

Trichonotidae .............................76

Tridensaspidae $\quad$.............................24

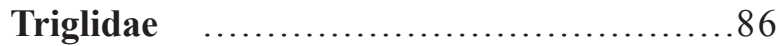

Trigonodontidae .............................. 81

Triodontidae ............................... 83

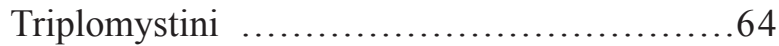

Triportheidae $\quad$.............................66

Tripterygiidae ............................. 80

Trispinachidae $\quad$................................ 85

Trissolepidae .................................48

Trissolepididae .............................48

Tristichopteridae (...........................91

Tristychiidae ................................40

Trygonorrhinidae ............................45

Tselfatidae ....................................61

Tubalepididae ..............................28

Tungusichthyidae ...........................56

Turboscinetinae ...............................53

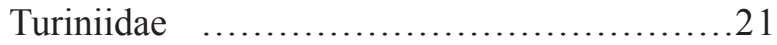

Turkmenidae ............................... 71

Turseoidae .....................................49

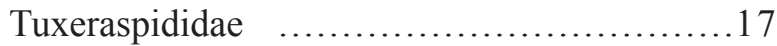

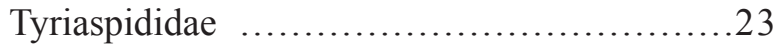

\section{$\mathbf{U}$}

Uarbryichthyidae .............................55

Uighuroniscidae .............................46

Umbridae ...................................68

Unamichthyidae ............................60

Undinidae ................................... 87

Uranolophidae ............................... 88

Uranoscopidae ............................... 81

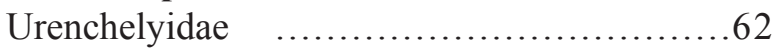

Urolophidae ...............................45

Uronemidae .................................99

Uropterygidae $\quad$............................50 
VAN DER LAAN R., Family-group names of fossil fishes

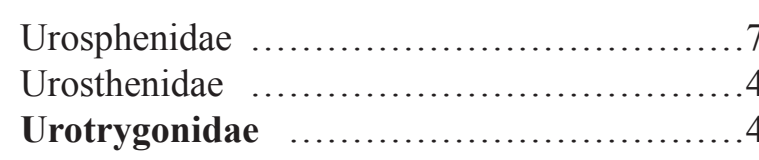

V

Vaillantellidae

Valenciidae

Varasichthyidae

Varialepididae

Veliferidae

Venusichthyidae

Vesperaliidae

Vidalamiinae

Vilulichthyinae

Vinctiferidae

Vomeropsinae

Vorhisiidae

\section{W}

Wakinichthiidae

Wakinichthyidae

Wapitiodidae

Weejasperaspidae

Weigeltaspidae

Weigeltaspiden

Welcommiidae

Wellingtonellidae

Whiteichthyidae

Whiteiidae

Williamsaspidae

Willomorichthyidae

Witaaspididae

Wudinolepidae

Wuttagoonaspidae

Wuttagoonaspididae

\section{$75 \quad \mathbf{X}$}

48

45

\section{$X$}

Xenacanthi ..36 36,40

Xenocyprididae ............................65

Xenosynechodontidae .......................40

.79 Xiphiidae .....................................77

.59 Xiphiorhynchidae ............................77

.48 Xiphodolamiidae ............................42

.71 Xipodolamiinae ..............................42

.54 Xiushuiaspidae .............................24

.34 Xiushuiaspididae ..........................24

.57

91

.57

.77

.66

Yunnanolepididae
Yunnanolepidinae

$.63 \mathrm{Z}$

.63

40

.26

17

17

.41

34

48

87

30

49

.23

28

29

.29

\section{Y}

Yelangichthyidae .............................50

Youngolepididae .............................88

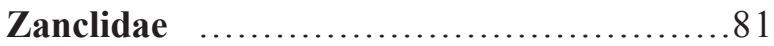

Zaniolepididae $\ldots \ldots \ldots \ldots \ldots \ldots \ldots \ldots \ldots \ldots$

Zanobatidae .................................45

Zaphlegidae $\quad$............................... 74

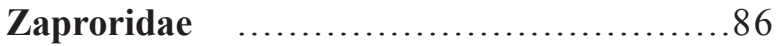

Zeidae ....................................72

Zenarchopteridae .........................79

Zenaspididae ...............................23

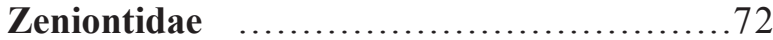

Zhaotongaspididae ..........................25

Zignoichthyidae ............................ 83

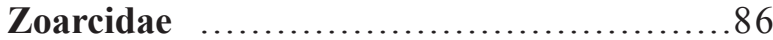

Zorzinichthyidae $\quad \ldots \ldots \ldots \ldots \ldots \ldots \ldots \ldots \ldots \ldots 1$

Zygzabatidae ..............................46 